University of Louisville

ThinkIR: The University of Louisville's Institutional Repository

Electronic Theses and Dissertations

6-1948

\title{
A study of child health facilities in Kentucky.
}

Jess L. Cusick

University of Louisville

Follow this and additional works at: https://ir.library.louisville.edu/etd

Part of the Social Work Commons

\section{Recommended Citation}

Cusick, Jess L., "A study of child health facilities in Kentucky." (1948). Electronic Theses and Dissertations. Paper 1891.

https://doi.org/10.18297/etd/1891

This Master's Thesis is brought to you for free and open access by ThinkIR: The University of Louisville's Institutional Repository. It has been accepted for inclusion in Electronic Theses and Dissertations by an authorized administrator of ThinkIR: The University of Louisville's Institutional Repository. This title appears here courtesy of the author, who has retained all other copyrights. For more information, please contact thinkir@louisville.edu. 


\title{
UNIVERSTTY OF LOUISVILLE
}

A STUDY OF CHILD HEALTH FACILITIES IN KENTUCKY

\author{
A Dissertation \\ Submitted to the Faculty \\ Of the Raymond A. Kent School of Social Work \\ In Partial Fulfillment of the \\ Requirements for the Degree \\ Of Master of Science in Social Work
}

\begin{abstract}
By
Jess L. Cusick Jr.
\end{abstract}

1948 
NAME OF STUDENT: Jess L. Cusick Jr.

TITLE OF THESIS: A Study of Child Health Facilities

In Kentucky

$\therefore$

APPROVEI BY READING COMMITIEE COMPOSED OF THE FOLLOWING

MEMBERS:

Howell V. Williams

Helen A. Brown

NAME OF DEAN: Howell V. Williams

DATE: $\frac{9 \text { fenen } 1248}{12}$ 
TABLE OF CONTENTS

Page

LIST OF TABLES . . . . . . . . . . . . . . .

LIST OF ILUUSTRATIONS . . . . . . . . . . . . . xii

Chapter

I. INTRODUCTION . . . . . . . . . . . . 1

II. PRIVATE PRACTICE . . . . . . . . . . 14

III. HOSFITAL FACILITIES AND SERVICIS . . . . . . 22

IV. COMMUNITY HEALTH SERVICES . . . . . . . . . 29

V. TOTAL VOLUME OF MEDICAL CARE . . . . . . . 34

VI. SUMMARY ................... 37

VII. CONCLUSIONS ................ 41

APPENDIX A. DETAILED TABULAR INFORMATION ON CHILD HEALTH

FACILITIES IN KENTUCKY . . . . . . .

Table

1. Children Under Medical Care on an Average Day by County Group in Kentucky ...........

2. Children Other than Newborn Receiving Medical Service for Health Supervision on an Average Day by County Group in Kentucky ............

3. Infants and Preschool Children Receiving Medical

Service for Health Supervision on an Average Day by County Group in Kentucky ...........

4. Chilaren Under Dental Care on an Average Day by County Group in Kentucky ........... 
5. Physicians and Dentists in Private Practice in

Kentucky by Type of Specialty, Certification by

American Specialty Boards and County Group as of

September, 1946 . . . . . . . . .

6. Physicians and Dentists in Private Fractice by Type of Specialty in Each County and Each City with 10,000 or more Population in Kentucky .....5 50

7. Availability of Physicians and Dentists in Private Practice by County Group in Kentucky . . . . . . 56

8. Visits per Day for Sick and Well Children by General Medical Practitioners, Pediatricians, and Other Specialists by County Groups in Kentucky ....

9. Children's Visits per Day by Dental Practitioners, Pedodontists, Orthodontists, and Other Specialists by County Group in Kentucky . . . . . . . . 58

12. Age, Race and Sex of Physicians in Private Practice by County Group in Kentucky . . . . . . . . . 59

13. Medical Training of General Practitioners by Age and County Group in Kentucky . . . . . . . . . 60

14. Availability to General Practitioners and Pediatricians of Hospitals Admitting ChIldren by County Group in Kentucky.................... 61

15. Summary of Data on Physicians in Kentucky ...... 62

16. Summary of Data on Pediatricians in Kentucky .... 63

17. Age, Race and Sex of Dentists in Private Practice by County Group in Kentucky . . . . . . . . . . 
19. Summary of Data on Dentists in Kentucky . . . . . 65

20. Number of Hospitals in Study by Type, Size and County Group in Kentucky ..... . . . . . 67

21. Hospital Facilities and Services for Newborn by Hospital Size and County Group in Kentucky . . . . 68

22. Facilities and Services for Children in General Hospitals by Hospital Size and County Group in Kentucky ... . . . . . . . . . . . 69

23. Child Admission Rates in General Hospitals by Hospital Size and County Group in Kentucky . . . . 70

24. Hospitals Admitting Acute Poliomyelitis Cases by Hospital Size and County Group in Kentucky . . . . . 71

26. Characteristics of Small General Hospitals $(5-24$ Beds) by County Group in Kentucky ......

27. Characteristics of Care for Newborn Infants in Larger General Hospitals (25 or more beds) in Kentucky ... . . . . . . . . . . . . .

28. Characteristics of Care for Sick Children in Larger General Hospitals (25 or more beds) in Kentucky . • 74

29. Child Admissions in Larger General Hospitals (25 or more beds) with Specified Characteristics by County Group in Kentucky ........... . . . . 75

31. Name and Location of General Hospitals in Study in Kentucky . . . . . . . . . . . . . . .

32. Summary of Selected Data Related to Child Care in 97 Individual General Hospitals in Kentucky . . . . 
33. Summary of Selected Data Related to Care of New-

born in 97 Individual General Hospitals in Kentucky ..

34. Name and Location of Special Hospitals in Study by Type of Hospital in Kentucky . . . . . . . . 82

35. Outpatient Services for Children by County Group in Kentucky .................... 84

37. Community Health Services for Children liuring One

Year by County and Each City of 50,000 or more Population in Kentucky ............. 85

38. Sessions of Medical Well-child Conferences During One Year by County Groups in Kentucky ......... 91

39. Patients and Visits to Medical Well-child Conferences During One Year by County Group in Kentucky . . . . 92

40. Immunizations Given Routinely in Medical Well-child

Conferences During One Year by County Group in Kentucky .................. 93

41. Staff of Medical Well-child Conferences by County Group in Kentucky . . . . . . . . . . . . 94

42. Practices in Medical Well-child Conferences by County Group in Kentucky ................. 95

43. Patients and Visits to Children's Dental Clinics During One Year by County Group in Kentucky . . . . . 96

43a. Type of Services in Children's Dental Clinics During One Year by County Group in Kentucky . . . . . . 97 
Table

44. Rates of Care in Children's Dental Clinics During

One Year by County: Group in Kentucky . . . . . . 98

45. Mental Hygiene Services for Children During One

Year in Kentucky . . . . . . . . . . . . 99

46. Services for Physically Handicapped Children During One

Year in Kentucky ... . . . . . . . . . 100

47. Children Immunized by Community Health Agencies Dur-

ing One Year by County Group in Kentucky . . . . . 101

48. Children 5-14 Years of Age in Counties without

School Health Services in Public Flementary Schools

by County Groups in Kentucky ............ 102

49. Staff Engaged in School Health Services by County

Group in Kentucky ................. 103

50. Services for Children Provided by official and

Voluntary Public Health Nursing Agencies During

One Year by County Group in Kentucky . . . . . . 104

51. Child Population, Estimated as of July 1, 1945, by

County Group and by Individual County in Kentucky . 105

66. Report Year for Health Services in Kentucky . . . 108

67. Alphabetical List of Kentucky Counties According to

Population and Proximity to Densely Populated Areas . 109

APPENDIX B. NATIONAL POPULATION DATA ANI VITAL STATISTICS • • 111

Table

1. Child Population Under 15, United States and Each

State, 1945 ............... 112 
2. Age Adjusted Death Rates Per 1000 Population by

United States and Each State . . . . . . . . 114

3. Infant Mortality Rate by Race, United States and Each State, 1945 . . . . . . . . . . 116

4. Maternal Mortality Rates by Race, United States and Each State, 1945 . . . . . . . . . 118

5. Fer Cent of Live Births in Hospitals, United States and Each State, 1935-1945 . . . . . . . . 120

6. Per Cent of Live Births in Hospitals by County Group, United States and Sach State, 1945 . . . . 122

7. Per Cent of Live Births in Hospitals by Race, United States and Each State 1935, 1939, 1941 and 1945 . . 124 APPENDIX C . SCHEDULES USED IN SURVEY . . . . . . . . 125 Schedule

I-A. Information on Pediatric Hospitals, General Hospitals and Other Hospitals with Maternity Facilities . . . 126

I-B. Information on Pediatric Hospitals, Ceneral Hospitals and Other Hospitals with Maternity Facilities . . 130

I-D. Supplementary Information on General, Pediatric, and Maternity Hospitals with 25 or more Beds . . . . 134

I-E. Nervous and Mental Hospitals, Including Institutions for the Feeble-minded . . . . . . . . . . 138

I-F. Tuberculosis Hospitals; Sanitoriums, and Preventoriums Admitting Tuberculous Patients . . . . . . 142

I-G. Convalescent and Chronic Hospitals, Rest Homes and Nursing Homes .................. 146 
Schedule

I-H. Contagious Disease Hospitals and General Hospitals

of 100 Beds or more . . . . . . . . . 150

I-J. Orthopedic Hospitals . . . . . . . . . 154

II. Summary of Public Health Services Available for

Children ........................ 158

II-A. Medical Well-child Conferences .......... 160

II-B. Dental Services for Children . . . . . . . . 162

II-C. Mental Hygiene Services for Children ....... 163

II-E. Communicable Disease Control . . . . . . . . 164

II-F. School Health Services . . . . . . . . . 165

II-G. Public Health Nursing Services . . . . . . 167

III-A. Physicians in Private Practice ........ 168

III-B. Pediatricians in Private Practice . . . . . 169

III-C. Dentists in Private Practice . . . . . . . . 172 BIBLIOGRAPHY . . . . . . . . . . . . 173 


\section{LIST OF TABLES}

Table

1. Per Capita Buying Income; United States and Each State Average $1944-1946$. . . . . . . . . 7

2. Children Per Physician by County Group . . . . . 14

3. Number of Specialists by Type and Certification . . - 15

4. Physicians Visits Per 1,000 Children Per Day by County Group ................ 16

5. Visits in State and County Groups by Type of Practitioner ................. 17

6. Visits by General Practitioners on One Day by Age of Patient................. 18

7. Location of Visits by Type of Physician . . . . 18

8. Type of Physician by Proportion of Health Supervision visits ............... 19

9. Number of Children per Dentist by County Group . . 20

10. Visits by Dentists per 1,000 Children Per Day by County Group ................. 20

11. Dental Services on One Day of 287 Dentists by Children's Age Groups ............... 21

12. Hospital Beds Per 1,000 Children by State and County Group ..................... 23

13. Admissions to Hospitals of 25 or More Beds by State and County Group .................. 
14. Births Occurring in Hospitals with 25 or More Beds with Specified Facilities by State . . . . . . . 26

15. Hospitals with Specified Facilities by Size . . . - 26

16. Days of Care in Special Hospitals by Type of Hospital. 27

17. Hours Per Month of Private Physicians in Community Health Service . . . . . . . . . . 30

18. Number of Clinics, Patients, and Visits Per 1,000 Children Under 5 Years of Age During One Year by State and County Group .............. 30

19. Rates for Specified Community Health Service Visits Per 1,000 Children Per Year by State ........ 32

20. Number of Children Per Public Health Nurse by State and County Group .. . . . . . . . . . . 32

21. Immunizations by Community Health Agencies Fer 1,000 Children in One Year by Specific Diseases . . . . 33

22. Total Volume of Medical Care Per 1,000 Children on One Day by State and County Group . . . . . . 35

23. Number of Children Fer 1,000 Under Dental Care on One Day by State and County Group . . . . . . . . 36 


\section{LIST OF ILLUSIRATIONS}

Figure

1. Classification of Kentucky Counties According to Population and Proximity to Densely Populated Areas . .

2. Trends of Maternal Mortality Rates Per 1,000 Live Births; United States and Kentucky 1915 - 1945 . . . 11

3. Trends of Infant and Neonatal Mortality Rates Per 1,000 Live Births; United States and Kentucky 1915 - 1945 • - 12 


\section{CHAPTER I}

\section{INTRODUCTION}

This study had its beginning in 1944 when the American Academy of Pediatrics passed a resolution "to make available to all mothers and children of the United States all essential preventive, diagnostic and curative services of high quality which used in cooperation with other services for children will make this country an ideal place for children to grow into responsible citizens." ${ }^{1}$ They realized that the first requisite to accomplishing their objective was a comprehensive knowledge of available facilities. They initiated a national survey of child health facilities through the various state Academies and Societies of Pediatries.

Dr. W. W. Nicholson, President of the Kentucky Pediatric Society, directed the gathering of the data used in this study for the State of Kentucky and has the over-all responsibility for the presentation of this report. He was assisted by Dr. W. B. Atkinson, Executive Secretary of the Kentucky Survey and his staff.

Continuing support and advice on the survey has been given by the Kentucky State Department of Health, Dr. P. E. Blackerby, Commissioner, particularly by Dr. W. B. Atkinson's Division of Nedical, Hospital and Related Services, and by Dr. Alice D. Chenoweth's Division of

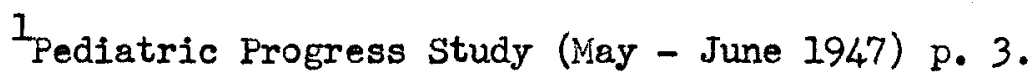


Maternal and Child Health.

The National Survey was directed by Dr. John P. Hubbard. The U. S. Public Health Service and the U. S. Children's Bureau contributed generously of their personnel and equipment in compiling and tabulating the data.

The writer prepared the textual material and analyses from the statistical tables compiled from the survey.

The report will set forth the results of a survey of the facilities and services currently available for the health and medical care of children in Kentucky. The sources of information for the survey were: (a) physicians and dentists; (b) voluntary and official community health agencies; and (c) all hospitals admitting children or maternity cases. 1

Eighteen schedules were used to cover these three sources of information. Some of the schedules were mail questionnaires. Others, requiring a field visit, were completed through personal visits by Dr. Nicholson, the State Chairman, the field staff, and the pediatricians themselves. In the beginnirig, the state Chairman traveled throughout the state, called upon each member of the Pediatric Society and explained to each his share in the study. These contacts were made in accordance with the original plan, which was based on the premise that those who were active in rendering child care should share not only in future planning but also in the collection of the data required to establish a sound basis for the improvement of child health services. Each member of

1

All of the data collected in the survey has not been used in this study. The national report will take up some of the broader aspects which cannot be dealt with in a single state because of the small numbers involved. 
the Society was therefore asked (a) to fill out his own schedule accurately and completely, (b) to assist in obtaining information from hospitals, (c) to co-operate with the local health officers in collecting the data pertaining to community health services, and (d) to contact general practitioners and specialists in his vicinity in order to assure a maximum response. The participation of the medical profession, particularly those recognized within their own communities as specialists in child care, can be considered as largely responsible for the gratifying response. The schedules were distributed in May, 1946.

The record for physicians' visits were obtained for a single day, one-seventh of the physicians reporting for each day in the week. Correction was made for the season in which the study was conducted. For non-reporting physicians, adjustment was made on the basis of a special study made in four states; so, unless otherwise indicated, the figures represent services for all practitioners in the state or specified area. The records for pediatricians cover 28 days. Schedules for community health services and hospitals cover one year.

Because of the absence of any adequate population data for 1946, the year of the study, special estimates of the child population as of July I, 1945 were made for each county (Appendix A, page 105). In this report "children" unless otherwise specified, refers to persons under 15 years of age, Incluaing new-born and premature infants.

Most of the data obtained, especially that which can be expressed in terms of rates per 1000 children, are measures of quantity of service rather than quality. Some estimates as to quality were attempted on the basis of certain objective criteria as to services available but on the whole quality of care was not assessed. 
The tables presented in Appendix A are listed according to the number given them in the national survey tabulation. Certain numbers are omitted from the sequence because they were not applicable to Kentucky for various reasons.

At this time, the data for all the states was not available for comperison. Eight states were selected to form a fairly representative group and have been used as a besis for some comparisons with Kentucky. "In selecting these states an attempt has been made to obtain an approximate sample of the whole country based on such considerations as geographic distribution and the size of state, per capita income, population distribution between metropolitan and rural counties, and the number of physicians and hospital beds available. This group of states is not a true sample, for the selection was conditioned by the necessity of having to exclude states that had not yet finished collecting their data. ${ }^{1}$

The states selected were: Oregon, Montana, New Mexico, Illinois, Alabama, North Carolina, New Hampshire, and Varyland with the District of Columbia and two counties of Virginia. These eight states had about five and one-half million children, or approximately 15 per cent of the nation's children. 2

One of the primary purposes of this study has been to determine the distribution of health and medical service so that existing inequalities in Kentucky might be defined in specific terms. To meet this,

1

Charles L. Williams, John Hubbard, Katherine Bain, "Community Health Services for Children in Eight Selected States", Journal of Public Health XXXVIII (January 1948), p. 76.

2 Williams, Hubberd and Bain, Op. Cit., p. 76. 


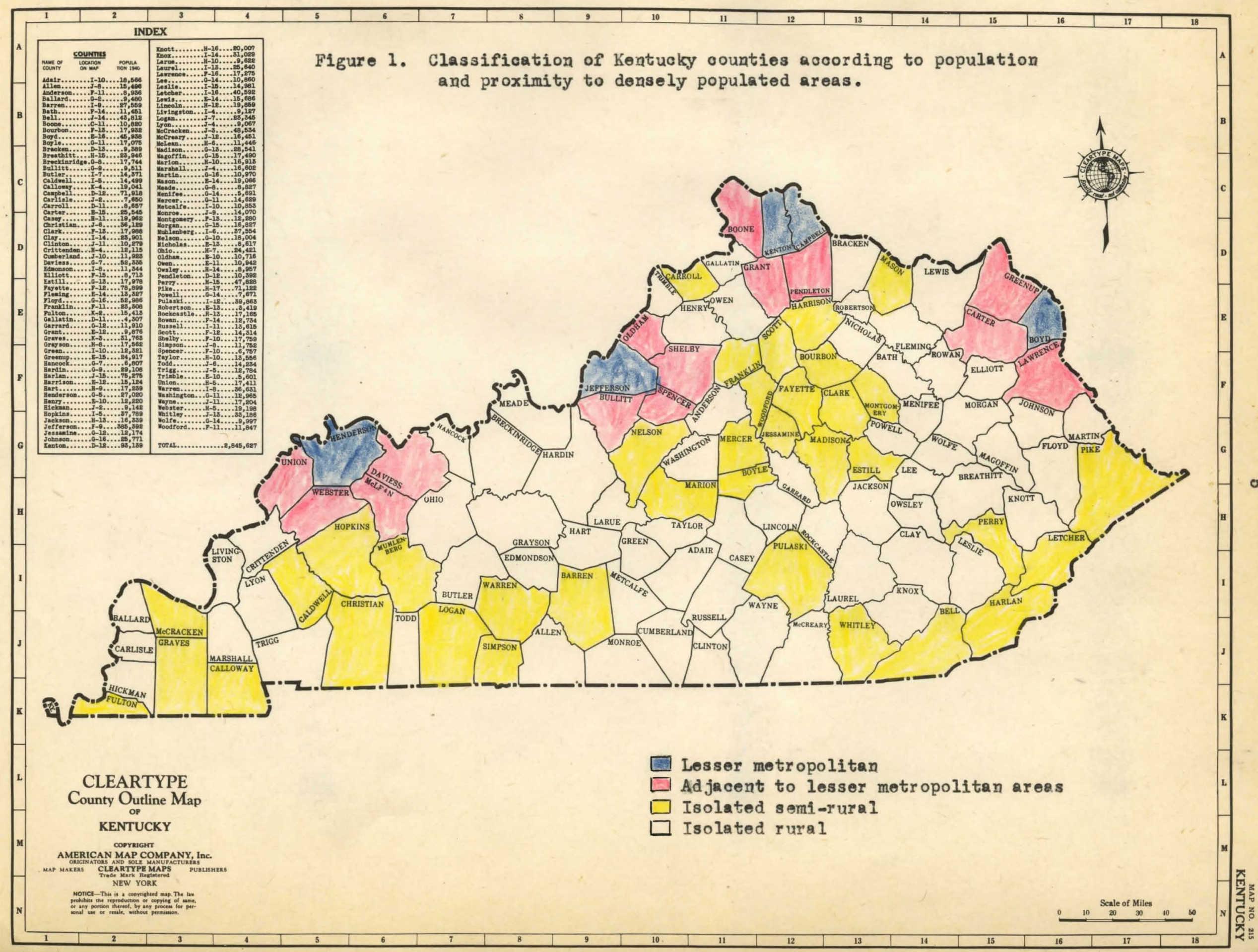


counties have been grouped to bring out contrasts between the more densely populated urban centers and the sparsely settled rural counties. The usual classification of urban and rural was not satisfactory in this case, since people cross county lines to get medical care in nearby centers. Counties have therefore been grouped together on the basis of two fundamental characteristics; (a) population density; and (b) proximity to densely populated areas. In this way separate consideration is given to counties which, although they themselves may be sparsely populated, are nevertheless relatively close to the metropolitan counties and the medical facilities there available. Under this classification, metropolitan counties are those which include districts of cities of 50,000 or more population. ${ }^{1}$ Counties which are contiguous to any of the metropolitan counties are classified as adjacent. Counties that do not touch any part of a metropolitan county have been termed isolated, and sub-divided into those with an incorporated place of 2500 or more population (semi-rural) and those without such a place (rural).

The overall picture of the situation of children in Kentucky, which forms the background for the survey, emerges from the following facts and figures. The child born in this state, starts out at a disadvantage. Kentucky, in comparison to other states, has a low economic classification. In an article on "Comparative Health Factors Among the States", Kentucky was ranked 40th in economic resources. ${ }^{2}$ Table 1 shows

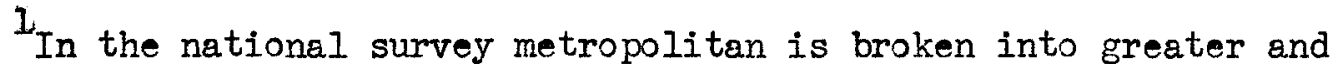
lesser metropolitan on the basis of greater or less than 1,000,000 population. In Kentucky since there are no areas greater than 1,000,000 they will only be referred to as metropolitan.

2 Gerhard Hirschfeld and Carl W. Strow, "Comparative Health Factors Among the States", American Sociological Review, XI (February 1946), p. 50. 
TABLE 1

PER CAPITA BUYING INCOME; UNITED STATES AND EACH STATE AVERAGE, 1944 - 1946

\begin{tabular}{|c|c|c|c|c|}
\hline State & $\begin{array}{l}\text { Total } \\
\text { Buying a } \\
\text { Income } \\
\text { (Millions of } \\
\text { Dollars) } \\
\text { (1) }\end{array}$ & $\begin{array}{l}\text { Fopulation } \\
\text { (Thousands) } \\
\text { (2) }\end{array}$ & $\begin{array}{l}\text { Per } \\
\text { Capita } \\
\text { Buying } \\
\text { Income } \\
\text { (Dollars) } \\
\text { (3) }\end{array}$ & $\begin{array}{r}\text { Rank } \\
\text { (4) }\end{array}$ \\
\hline United States ...... & 462,254 & 405,103 & 1,141 & - \\
\hline Alabama ......... & 5,810 & 8,278 & 702 & 45 \\
\hline Arizona $\ldots . . . .$. & 1,820 & 1,817 & 1,002 & 31 \\
\hline Arkansas ........ & 3,374 & 5,613 & 601 & 48 \\
\hline California ....... & 39,776 & 27,450 & 1,449 & 6 \\
\hline Colorado .......... & 3,935 & 3,328 & 1,182 & 17 \\
\hline Connecticut ...... & 8,840 & 5,599 & 1,578 & 2 \\
\hline Delaware ........ & 1,274 & 837 & 1,522 & 3 \\
\hline District of Columbia & 4,763 & 2,703 & 1,762 & 1 \\
\hline Florida ......... & 6,808 & 6,992 & 974 & 34 \\
\hline Georgia ......... & 7,159 & 9,280 & 771 & 42 \\
\hline Idaho .......... & 1,665 & 1,465 & 1,137 & 20 \\
\hline Illinois ......... & 31,418 & 23,163 & 1,356 & 10 \\
\hline Indiana ......... & 12,366 & 10,801 & 1,145 & 19 \\
\hline Iowe ............. & 7,724 & 7,287 & 1,060 & 25 \\
\hline Kansas .......... & 6,003 & 5,447 & 1,102 & 22 \\
\hline Kentucky ......... & 5,629 & 8,036 & 700 & 46 \\
\hline Louisiana ....... & 5,978 & 7,370 & 811 & 39 \\
\hline Maine ............ & 2,632 & 2,482 & 1,060 & 25 \\
\hline
\end{tabular}


TABLE 1 - Continued

\begin{tabular}{|c|c|c|c|c|}
\hline State & $\begin{array}{l}\text { Total } \\
\text { Buying } \\
\text { Income } \\
\\
\text { (Millions of } \\
\text { Dollars) }\end{array}$ & $\begin{array}{l}\text { Population } \\
\text { (Thousands) }\end{array}$ & $\begin{array}{l}\text { Per } \\
\text { Capita } \\
\text { Buying } \\
\text { Income } \\
\text { (Dollars) }\end{array}$ & Rank \\
\hline Maryland ........ & 7,790 & 6,324 & 1,232 & 15 \\
\hline Massachusetts .... & 16,733 & 12,978 & 1,289 & 12 \\
\hline Michigan ........ & 21,769 & 17,142 & 1,270 & 13 \\
\hline Minnesota ........ & 7,881 & 8,065 & 977 & 33 \\
\hline Mississippi ...... & 3,587 & 6,419 & 559 & 49 \\
\hline Missouri ........ & 11,240 & 10,955 & 1,026 & 29 \\
\hline Montana .......... & 1,693 & 1,385 & 1,222 & 16 \\
\hline Nebraska ......... & 3,987 & 3,737 & 1,067 & 24 \\
\hline Nevada ........... & 648 & 427 & 1,518 & 4 \\
\hline New Hampshire .... & 1,307 & 1,454 & 899 & 37 \\
\hline New Jersey ....... & 17,585 & 12,484 & 1,409 & 9 \\
\hline New Mexico ....... & 1,211 & 1,589 & 762 & 43 \\
\hline New York ........ & 58,910 & 39,271 & 1,500 & 5 \\
\hline North Carolina ... & 7,603 & 10,812 & 703 & 44 \\
\hline North Dakota ..... & 1,652 & 1,560 & 1,059 & 27 \\
\hline Ohio ............ & 27,730 & 21,224 & 1,306 & 11 \\
\hline Oklahoma ......... & 5,483 & 6,401 & 857 & 38 \\
\hline Oregon ........... & 5,159 & 4,084 & 1,263 & 14 \\
\hline Pennsylvania ..... & 33,308 & 28,620 & 1,164 & 18 \\
\hline Rhode Island ..... & 3,091 & 2,179 & 1,419 & 7 \\
\hline South Carolina ,.. & 3,812 & 5,763 & 661 & 47 \\
\hline South Dakota ..... & 1,657 & 1,619 & 1,023 & 30 \\
\hline
\end{tabular}


TABLE 1 - Continued

\begin{tabular}{|c|c|c|c|c|}
\hline State & $\begin{array}{l}\text { Total } \\
\text { Buying } \\
\text { Income } \\
\text { (Nillions of } \\
\text { Dollars) }\end{array}$ & $\begin{array}{l}\text { Population } \\
\text { (Thousands) }\end{array}$ & $\begin{array}{l}\text { Per } \\
\text { Capita } \\
\text { Buying } \\
\text { Income } \\
\text { (Dollars) }\end{array}$ & Rank \\
\hline Tennessee ........ & 6,780 & 8,687 & 780 & 41 \\
\hline Texas ............ & 19,038 & 20,563 & 926 & 35 \\
\hline Utah ............. & 1,982 & 1,856 & 1,068 & 23 \\
\hline Vermont .......... & 993 & 997 & 996 & 32 \\
\hline Virginia ......... & 8,114 & 8,930 & 909 & 36 \\
\hline Washington ........ & 9,318 & 6,578 & 1,417 & 8 \\
\hline West Virginia .... & 4,195 & 5,210 & 805 & 40 \\
\hline Wisconsin ........ & 10,219 & 9,072 & 1,126 & 21 \\
\hline Wyoming .......... & 817 & 773 & 1,057 & 28 \\
\hline Maryland - T.c. ${ }^{c} .$. & 12,553 & 9,027 & 1,394 & \\
\hline
\end{tabular}

a. Sales Managernent: Vols. 54, 56, and 58; issue No. 10 of each volume. Copyright Sales Management, Inc.

b. Estimates by National Income Division, U.S. Department of Commerce (unpublished).

c. Excludes Arlington and Fairfax counties in Virginia.

that Kentucky's per capita income is $\$ 700$. This places it 46 th among the states in this respect.

Despite these facts or perhaps because of them, Kentucky has a comparatively large percentage of children in its population. The percentage (34.7 in 1945) is 12th highest in the country. Twenty per cent of the children were in metropolitan counties, 8 per cent in 
adjacent counties, 38 per cent in isolated semi-rural, and 34 per cent in isolated rural counties.

The state's death rate in 1940 was 30 th highest. It was 10.8 per thousand as compared with the overall rate in the United States of $10.7 .^{1}$

Out of every 1000 children born alive in Kentucky in $1945,46.9$ died during their first year of life. This places Kentucky as 40th in the nation. 2 Negro infant mortality is about 65 per cent higher than the rate for white infants: 70.4 as against 45.6 per 1000 . Figure 2 shows that there has been considerable improvement in the infant mortality rate. In 1917 the rate was about 90 out of every 1000 children born alive. By 1945 this rate was only about half as large.

Maternal mortality rates have also dropped during the last thirty years. From a rate of about 5 maternal deaths for every 1000 babies born alive in 1915 , it has dropped to 2.5 per 1000 live births in 1945. Figure 3 shows this decline graphically.

Kentucky had 44 per cent of its births occurring in hospitals in 1945, placing it 46th among the states. In the metropolitan counties the percentage was 84.8 ; in adjacent counties, 41.6; in isolated semi-rural;", 38.9; and in isolated rural, 20.8. The percentage of white births in hospitals is 44.3 and of negroes 35.3 . Despite the low rank Kentucky has in the nation, the increase in the last ten years from 9 per cent in 1935 to 44 per cent in 1945 is encouraging.

\section{${ }^{1}$ See Table 2 , Appendix B.}

2U. S. Children's Bureau Charts on Births, Infant and Childhood Mortality and Maternal Mortality, Chart IM-OI. 


\section{FIGURE 2}

TRENDS OF MATERNAL MORTALITY RATES PER 1,000 LIVE BIRTHS: UNITED STATES AND KENYUCKY, $1915-1945$

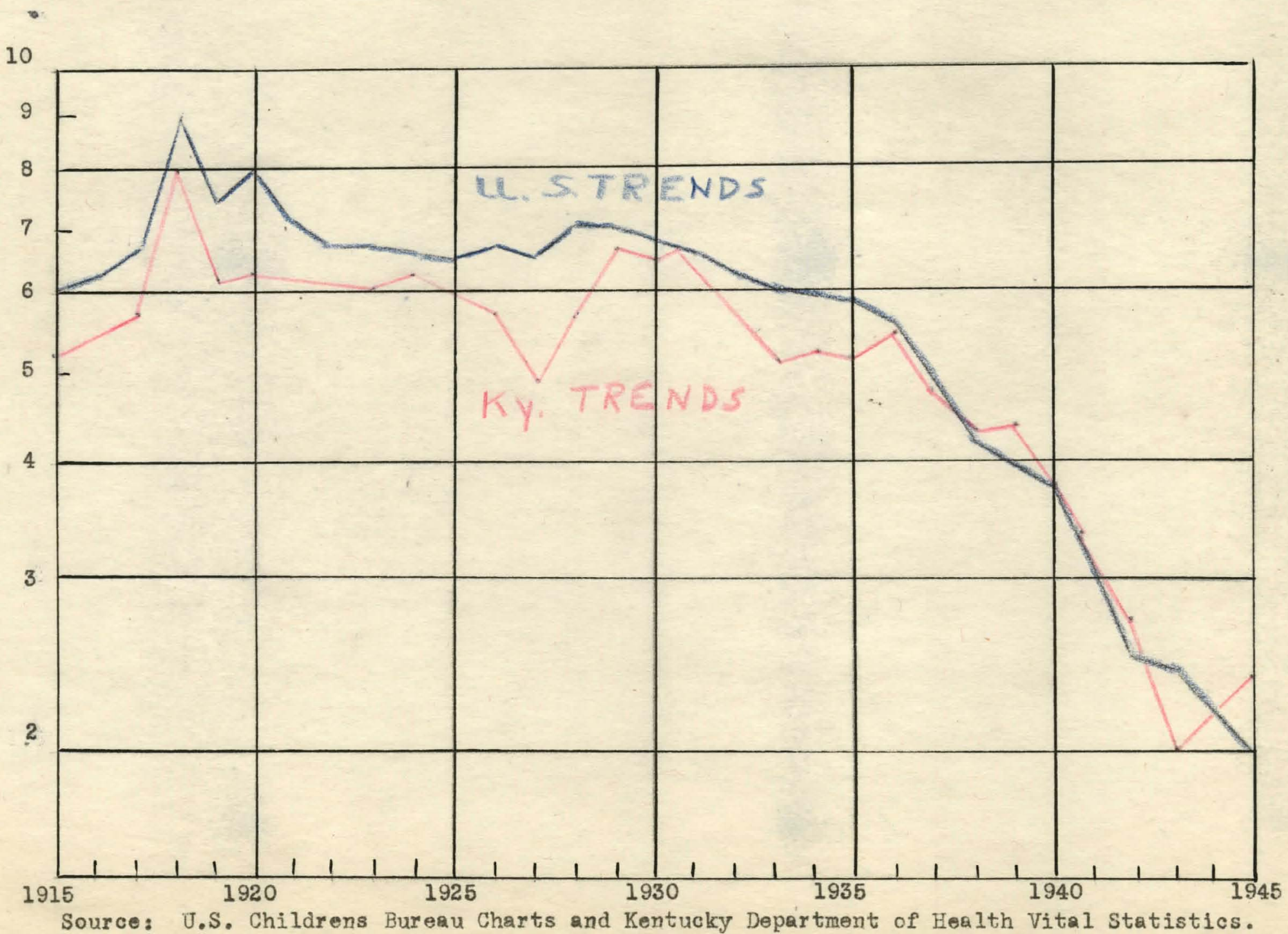


FIGURE 3

TRENDS OF INFANT AND NEONATAL MORTALITY RATES PER 1,000 LIVE BIRTHS: UNITED STATES AND KENTUCKY, 1915-1945

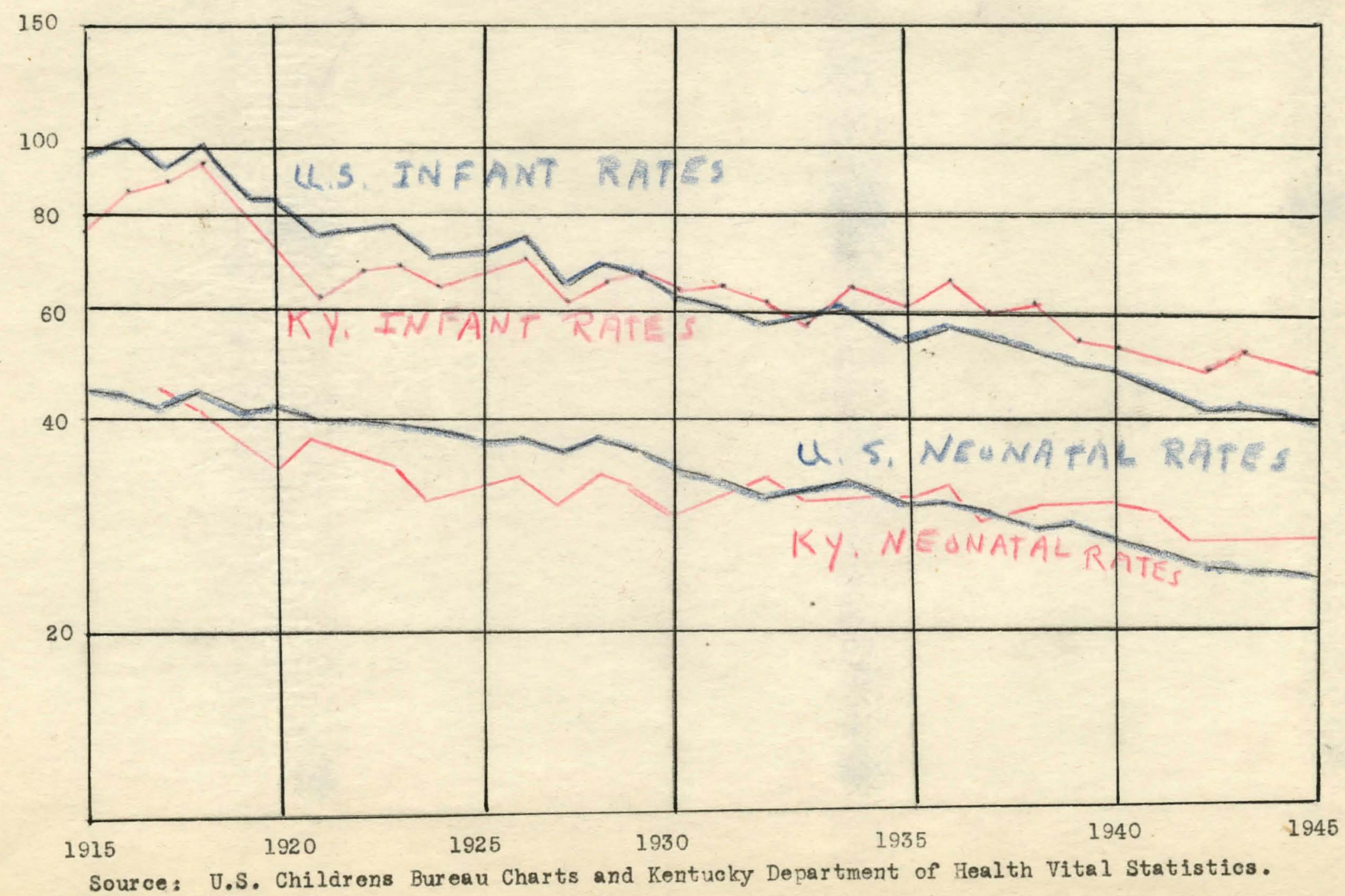


While Kentucky ranked 18th among the states in total births in 1945, its ability to care for children as measured by health facilities and economic resources is much below that of the other states. Hirschfeld and Strow rank Kentucky 46 th in health and sanitation and 4lst in economic and cultural factors. ${ }^{I}$ Kentucky thus ranks high as a producer of children and low in ability to offer them opportunity for development. 


\section{CHAPTER II}

\section{PRIVATE PRACTICE}

To indicate the accessibility of medical service for children, the ratio of physicians to children has been used. When this study was made in 1946 there were 1751 physicians in private practice in Kentucky, or one physician per 504 children. This compares unfavorably with the average ratio in the eight states of 338 children per physician and is far below the highest state which was 219 children per physician.

The number of children per physician in each of Kentucky's four county groups was as follows:

\section{TABLE 2}

CHILDEEN PER PHYSICIAN BY COUNTY GROUP

\begin{tabular}{l|c|c}
\hline & $\begin{array}{c}\text { Number of Children } \\
\text { Per Physician }\end{array}$ & $\begin{array}{c}\text { Actual Number } \\
\text { of Physicians }\end{array}$ \\
\hline Metropolitan $\ldots \ldots \ldots \ldots \ldots \ldots$ & 265 & 657 \\
Adjacent . . . . . . . . . . . . . & 617 & 117 \\
Isolated semi-rural ....... & 486 & 682 \\
Isolated rural .......... & 1082 & 295 \\
\hline
\end{tabular}

There are 63 counties classified isolated rural. These counties contain 34 per cent of Kentucky's children but only 17 per cent of her physicians. Thus the ratio of children to physicians is four times 
higher in those areas than in the metropolitan areas, a fact which speaks for itself of inadequate medical care.

The number of physicians in each county classified as to type of practice is shown in Appendix $A$, page 50.

At the time this survey was made there were 31 pediatricians in Kentucky or one per 28,500 children. In the eight states this ratio averaged 11,000 per pediatrician, with the best ratio being 5,300., Of these 31 pediatricians, 17 had been certified by the American Board of Pediatrics.

Most of these specialists were in the larger population centers, 16 of them in metropolitan counties, 14 in isolated semi-rural counties and one in isolated rural counties. Only 4 were in towns of less than 10,000 population.

Besides the pediatricians there were 514 other specialists practicing in Kentucky in 1946. Their number by type and certification follows: TABLE 3

NUMBER OF SPECIALISTS BY TYPE AND CERTIFICATION

\begin{tabular}{l|c|c}
\hline & $\begin{array}{c}\text { Total } \\
\text { Number }\end{array}$ & $\begin{array}{c}\text { Number Certified } \\
\text { by American } \\
\text { Specialty Boards }\end{array}$ \\
\hline Internal Medicine and Allied Specialties ... & 98 & 26 \\
Allergy ........................... & 2 & 1 \\
Psychiatry and Neurology ................ & 11 & 4 \\
Surgery, except Orthopedic ............... & 201 & 41 \\
Orthopedic surgery ..................... & 11 & 6 \\
Obstetrics and Gynecology ................. & 53 & 10 \\
Ophthalmology and otolaryngology .......... & 107 & 33 \\
Radiology and Anesthesiology .............. & 31 & 13 \\
\hline
\end{tabular}


A further breakdown of the figures collected on the qualifications of the state's doctors, reveals that of the 767 general practitioners who gave information about the length of their hosrital training, 30 per cent had none. Forty-two per cent had less than two years, and 28 per cent had more than two years. Sixty-six per cent had none or less than one month of hospital training in pediatrics.

of the 1751 private practitioners in Kentucky, 67 are negroes. There are 25 female physicians, 2 of whom are negroes. One pediatrician was a negro. There were four female pediatricians at that time. Twentysix per cent of all the doctors were over 65 years old; 36 per cent were less than 45 years of age. Of the pediatricians, 24 are under 45 years of age and none are more than 65.

The figures on the amount of service rendered children bir all private practitioners give a significant picture of the amount of care received by children in the various county groups. As might have been expected there were twice as many visits to children in the metropolitan as in the isolated rural counties.

TABLE 4

PHYSICIANS VISITS PER 1000 CHILUREN PER DAY BY COUNTY GROUP

\begin{tabular}{l|c|c}
\hline \hline & $\begin{array}{c}\text { Physicians } \\
\text { visits per 1000 } \\
\text { Children }\end{array}$ & $\begin{array}{c}\text { Percentage of } \\
\text { Child Population } \\
\text { in Each County } \\
\text { Group }\end{array}$ \\
\hline State .................... & 9.9 & 100 \\
Metropolitan Counties ....... & 13.9 & 20 \\
Adjacent Counties .......... & 8.6 & 8 \\
Isolated Semi-rural Counties. & 10.6 & 38 \\
Isolated Rural Counties ..... & 6.9 & 34 \\
\hline
\end{tabular}


General practitioners provide most of the care given by private physicians to children in Kentucky, their percentage of care drops considerably in metropolitan counties because of the relatively larger number of pediatricians and other specialists in those areas as is show in the following table:

\section{TABLE 5}

VISITS IN STATE AND COUNTY GROUPS BY TYPE OF PRACTITIONER

\begin{tabular}{l|c|c|c}
\hline & $\begin{array}{r}\text { General } \\
\text { Practitioner } \\
\text { (Per cent) }\end{array}$ & Pediatrician \\
(Fer cent) & $\begin{array}{c}\text { Other } \\
\text { Specialists } \\
\text { (Fer cent) }\end{array}$ \\
\hline State ............. & 82 & 7 & 11 \\
Metropolitan ...... & 64 & 14 & 22 \\
Adjacent ......... & 96 & 0 & 4 \\
Isolated Semi-rural & 81 & 8 & 11 \\
Isolated Rural .... & 98 & 1 & 1 \\
\hline
\end{tabular}

The general practitioner on the average saw 20 patients daily of which 6 were children, during the period covered by this study. Six per cent reported sesing more 50 patients dally. Table 6 sets forth the number of patients seen.

In contrast to the general practitioners, the pediatricians averaged, over a period of 28 days, 22 visits per day.

All types of private physicians see most of their patients in their office or the hospital. The general practitioner sees more patients at home than the specialists, who in turn see a considerably higher percentage of their patients in hospitals. 
TABLE 6

VISITS BY GENERAL PRACTITIONERS ON ONE DAY BY AGE OF PATIENT

\begin{tabular}{|c|c|c|}
\hline $\begin{array}{l}\text { Number of Visits } \\
\text { On One Day } \\
\text { (Per cent) }\end{array}$ & $\begin{array}{l}\text { Persons of } \\
\text { All Ages } \\
\text { (Fer cent) }\end{array}$ & $\begin{array}{c}\text { Children } \\
\text { Under } 15 \text { years } \\
\text { (Per cent) }\end{array}$ \\
\hline Non $\ldots \ldots \ldots \ldots \ldots$ & 14 & 20 \\
\hline $1-9 \ldots \ldots \ldots \ldots$ & 17 & 56 \\
\hline $10-19 \ldots \ldots \ldots \ldots$ & 24 & 20 \\
\hline $20-29 \ldots \ldots \ldots \ldots$ & 20 & \\
\hline $30-39 \ldots \ldots \ldots$ & $u_{4}$ & \\
\hline $40-49 \ldots \ldots \ldots \ldots$ & 5 & 4 \\
\hline $50-59 \ldots \ldots \ldots$ & 3 & \\
\hline 60 and over .......... & 3 & \\
\hline 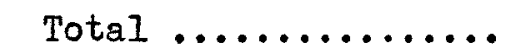 & 100 & 100 \\
\hline
\end{tabular}

TABLE 7

LOCATION OF VISITS BY TYPE OF PHYSICIAN

\begin{tabular}{|c|c|c|c|}
\hline & $\begin{array}{c}\text { General } \\
\text { Practitioner } \\
\text { (Per cent) }\end{array}$ & $\begin{array}{l}\text { Pediatrician } \\
\text { (Per cent) }\end{array}$ & $\begin{array}{c}\text { Other } \\
\text { Specialists } \\
\text { (Per cent) }\end{array}$ \\
\hline office...$\ldots \ldots \ldots$ & 66 & 61 & 60 \\
\hline Home,$\ldots \ldots \ldots \ldots \ldots$ & 22 & 15 & 4 \\
\hline Hospital .......... & 12 & 24 & 36 \\
\hline$A] 1 \ldots \ldots \ldots \ldots$ & 100 & 100 & 100 \\
\hline
\end{tabular}


Health supervision for well children has become increasingly accepted in recent years. Pediatricians now receive training in normal growth and development, feeding and care of well children, and the handling of the usual problems of social and emotional adjustment. General practitioners are also assuming considerable responsibility in health supervision of well children.

The principal load in child health supervision by private physicians in Kentucky is carried by general practitioners. Of the private physicians' visits to well children, 76 per cent were made by general practitioners, 17 per cent by pediatricians, and 7 per cent by other specialists.

Nineteen per cent of all general practitioners' visits to children are for health supervision; 49 per cent of pediatricians' visits were for this purpose. Comparison of Kentucky with the eight selected states is shown in Table 8.

TABLE 8

TYPE OF PHYSICIAN BY PROPORTION OF HEALTH SUPERVISION VISITS

\begin{tabular}{l|c|c|c|c}
\hline & & \multicolumn{3}{|c}{ Eight Selected States } \\
& Kentucky & Highest & Average & Lowest \\
\cline { 3 - 5 } & Ger cent) & (Per cent) & (Fer cent) & (Per cent) \\
\cline { 2 - 5 } General Practitioners & 19 & 33 & 26 & 19 \\
Pediatricians ....... & 49 & 61 & 54 & 33 \\
\hline
\end{tabular}

At the time of the survey there were 698 dentists in Kentucky. This was a ratio of one dentist for each 1263 children. This compared with 338 children per dentist in the highest state. The ratio by county 
groups is shown in Table 9. Nine counties had no dentist at all. TABLE 9

NUMBER OF CHILDREN PER DENTIST BY COUNTY GROUP

Number of Children

per Dentist

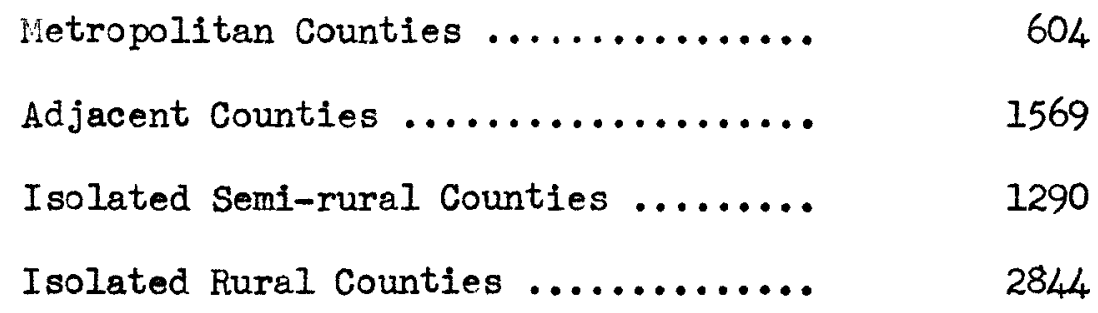

of the 698 dentists in Kentucky, 28 were negroes. There were 6 white and 1 female dentists at that time. Twelve per cent of the dentists were over 65 years old, 51 per cent between 45 and 64 , and 37 per cent under 45 years of age. Of 337 dentists reporting on office assistants, 74 had none, 202 had one, and 61 had 2 or more. Of 324 dentists reporting on dental hygienists, only 11 reported employing one. TABLE 10

VISITS BY LENTISTS PER 1,000 CHILDREN PER DAY BY COUNTY GROUP Metropolitan Counties ............ 2.7

Adjacent Counties ................ 1.4

Isolated Semi-rural Counties ......... 1.5

Isolated Rural Counties ............ 0.6

A dentist sees 8 or 9 patients on an average day, of whom 26 per cent are children. The ratio of fillings to extractions give some Indication of the amount of preventive care given by dentists. The relatively high ratios shown in Table 11 mean that children under the 
care of private dentists are receiving proper care.

TABLE 11

DENTAL SERVICES ON ONE DAY OF 287 DEITISTS

BY CHILDREN'S AGE GROUPS

\begin{tabular}{c|c|c}
\hline & $\begin{array}{c}\text { Children } \\
\text { Under 6 Years }\end{array}$ & $\begin{array}{c}\text { Children } \\
6-14 \text { Years }\end{array}$ \\
\hline Extractions .. & 63 & 179 \\
Fillings ..... & 99 & 433 \\
\hline
\end{tabular}

Out of 275 dentists, 25 reported rendering pre-school or school dental service during a month. Those participating averaged three and one-half hours per week on these services. Thirty-three reported other dental activities such as teaching and institutional work.

In February 1948 there were 2,381 private physicians practicing in Kentucky. This addition of about 600 doctors more than at the time of the survey in September 1946 is mainly accounted for by the return of men from the service. While this number makes some difference in the ratios of care to children, it probably makes little if any difference in the relative ranking with other states since there has been nothing to indicate that relatively more doctors returned to Kentucky than other states. 


\section{CHAPTER III}

\section{HOSPITAL FACILITIES AND SERVICES}

We turn now to a consideration of hospital facilities and services available to children in Kentucky. In this report the term"hospital"is limited to those institutions caring for chilaren, including the newborn. No institution having less than five beds for regular inpatient care is included, nor are federally owned hospitals included. For the purpose of this report "General" is taken to include maternal and pediatric hospitals. Hospitals which have five or more beds permanently set aside for the care of children are considered to have a pediatric unit.

There are 97 general hospitals in Kentucky. Twelve of these have pediatric units. Of these, 48 per cent have between 25 and 99 beds and 35 per cent have between 5 and 24 beds. The general hospitals covered by this survey have 6,088 beds or 6.9 per 1000 children. There are 378 beds set aside exclusively for children. In the following Table these data are compared with figures from the eight states used as a comparison.

Kentucky has approximately one third as many beds per 1000 children as the highest state; and within the state the rural counties have less than half as many beds as the metropolitan counties. Kentucky's metropolitan counties have slightly fewer beds than the average of the comparative states and since only 20 per cent of the children live in 
TABLE 12

HOSPIIAL BEDS PER 1000 CHILDREN BY STATE AND COUNTY GROUP

\begin{tabular}{|c|c|c|c|}
\hline & \multicolumn{2}{|c|}{ Per 1000 Children } & \multirow{2}{*}{$\begin{array}{l}\text { Percentage of beds } \\
\text { Which are Permanently } \\
\text { Set Aside for Children }\end{array}$} \\
\hline & $\begin{array}{l}\text { Total } \\
\text { Beds }\end{array}$ & $\begin{array}{c}\text { Pediatric } \\
\text { Beds }\end{array}$ & \\
\hline Kentucky ......... & 6.9 & .4 & 6.2 \\
\hline Metropolitan .... & 12.0 & - & - \\
\hline Rural ......... & $4 \cdot 9$ & - & - \\
\hline Highest state ..... & 20.6 & 2.0 & 9.8 \\
\hline Average State ..... & 12.5 & 1.2 & 9.4 \\
\hline Lowest State ...... & 5.7 & .5 & 4.3 \\
\hline
\end{tabular}

these counties, the remaining children obviously have inadequate hospital facilities available. The percentage of beds set aside for children is 30 per cent lower than the average of the other states.

Admissions of children to general hospitals during the year of the study totalled 24,164 or an annual rate of 27.4 per 1000 children. This compares with a rate of 83 per 1000 children in the highest of the eight states. In metropolitan and adjacent counties the rate was 46 per 1000, and in isolated counties it was 20 per 1000. This means that in the metropolitan counties of Kentucky the rate of admissions to hospitals is a little more than half that of a state with good medical care and that in the isolated counties of Kentucky admissions are less than one-quarter of that state. These facts speak eloquently for the need of hospital facilities in Kentucky.

Hospitals having between 25 and 99 beds accounted for 47.2 
per cent of the total admissions; 27.4 per cent were admitted to 250 or more bed hospitals ; 18 per cent to the $100-249$ bed group; and 7.4 per cent to the $5-24$ bed group.

of the 97 general hospitals, 4 are exclusively for negroes, and 49 admit both white and negro patients. Forty-four admit only white patients.

The newborn received 53 per cent of the total care of children rendered in general hospitals in Kentucky. There rere 1,109 bassinets and 134 incubators in Kentucky hospitals at the time of the study. In view of the rapid rate of increase of hospital births during the last ten years these facts are important in planning for the future.

The quality of care rendered by hospitals is difficult to assess. An effort was made, however, to obtain certain objective criteria as indices to quality. These included space, organization of pediatric service, medical staff, nursing, and certain accepted pediatric practices. The quantity or presence and absence of these services and practices give some index as to quality of service rendered.

The proportion of children admitted to hospitals with certain specified facilities in comparison with the highest of the eight states selected for comparison is. shown in Table 13.

It can readily be seen that Kentucky's hospitals are not able to give the type of care which could be available. Less than half of those hospitalized go to a hospital which has any house staff. In the highest state 90 per cent of children go to hospitals with house staff. Facilities in the 100 rural counties which contain 72 per cent of Kentucky's children are far below the metropolitan counties which in turn 
TABLE 13

CHILD ADMISSIONS TO HOSFITALS OF 25 OR MORE BEDS BY STATE AND COUNTY GROUFS

\begin{tabular}{|c|c|c|c|c|}
\hline Hospital Facilities & $\begin{array}{l}\text { Kentucky } \\
\text { (Per cent) }\end{array}$ & $\begin{array}{l}\text { Metropolitan } \\
\text { and Adjacent } \\
\text { (Per cent) }\end{array}$ & $\begin{array}{l}\text { Isolated } \\
\text { Counties } \\
\text { (Per cent) }\end{array}$ & $\begin{array}{l}\text { Highest } \\
\text { State } \\
\text { (Per cent) }\end{array}$ \\
\hline Separate pediatric unit ... & 48.3 & 72.1 & 25.5 & 91 \\
\hline $\begin{array}{l}\text { Graduate nurse on duty at } \\
\text { all times in pediatric unit }\end{array}$ & 26.1 & 47.5 & 7.0 & 71 \\
\hline House staff $\ldots \ldots \ldots \ldots \ldots$ & 48.8 & 78.3 & 19.7 & 90 \\
\hline Clinical laboratory ....... & 77.4 & 81.1 & 73.7 & 97 \\
\hline $\begin{array}{l}\text { Selected clinical labora- } \\
\text { tory services available ,.. }\end{array}$ & 66.0 & 76.0 & 56.0 & 96 \\
\hline Trained dietician on staff & 56.0 & 77.8 & 34.4 & 94 \\
\hline $\begin{array}{l}\text { Separate ward for infants } \\
\text { other than newborn ........ }\end{array}$ & 33.7 & 50.6 & 17.4 & 79 \\
\hline Average percentage ........ & 51.0. & 69.0 & 33.4 & 88 \\
\hline
\end{tabular}

are far below those of the highest state.

The percentage of newborn in hospitals with certain facilities is shown in Table 14 .

Hospitals which have between 5 and 24 beds receive 7.4 per cent of children's admissions to hospitals in Kentucky and 8.7 per cent of hospitalized births occur in them. Although these percentages are small the lack of facilities in these hospitals is a factor to be considered in planning for the future. Table 15 shows how these small hospitals compare with the larger ones in certain facilities. 
TABLE 14

BIRTHS OCCURRING IN HOSPITALS WITH 25 OR MORE BELS WITH SPECIFIED FACILITIFS BY STATE

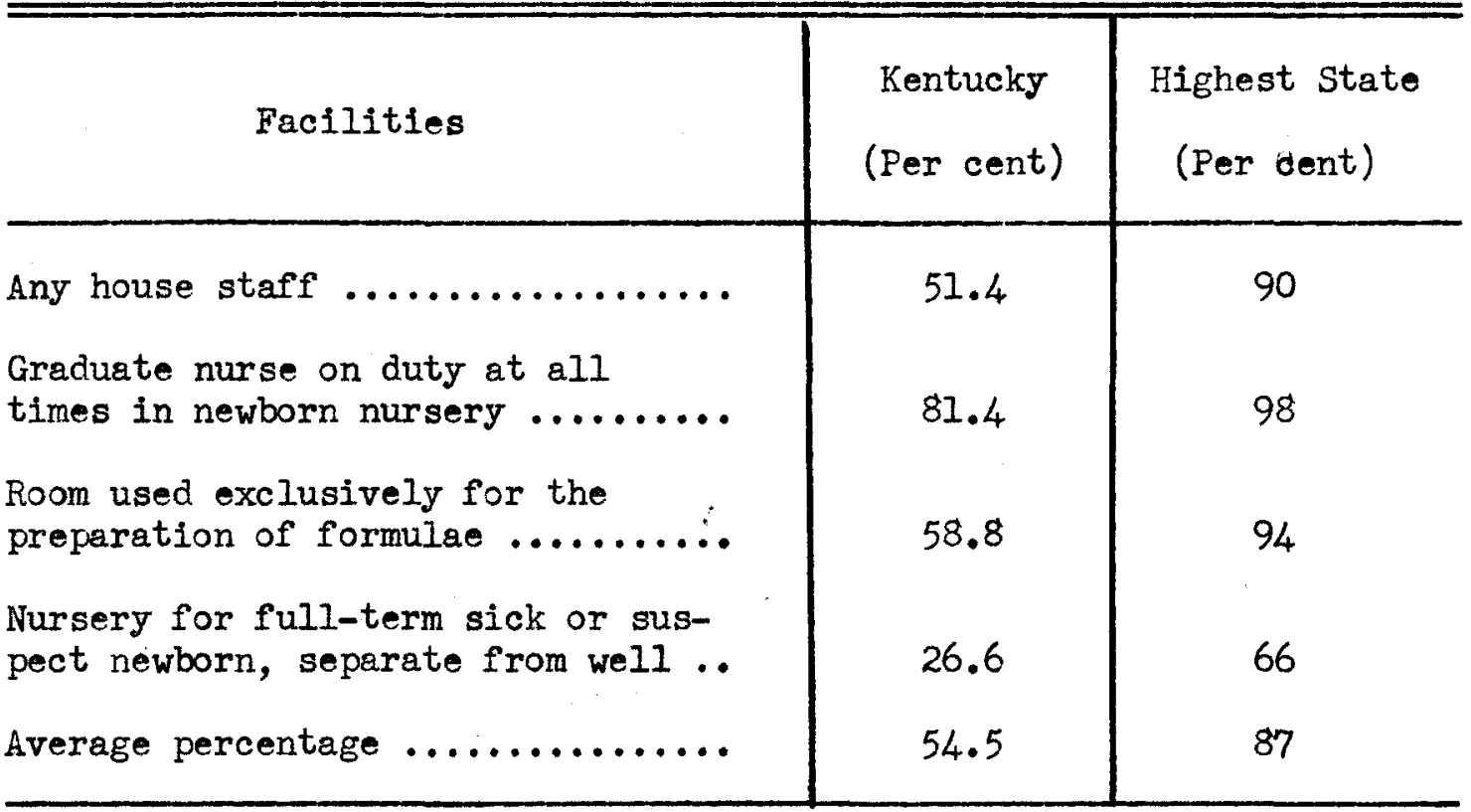

HOSPITALS WITH SPECIFIED FACILITIES BY SIZE

\begin{tabular}{|c|c|c|}
\hline Facilities & $\begin{array}{l}\text { Fewer Than } \\
25 \text { Beds } \\
\text { (Per cent) }\end{array}$ & $\begin{array}{l}\text { More Than } \\
25 \text { Beds } \\
\text { (Per cent) }\end{array}$ \\
\hline 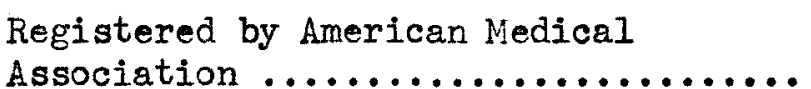 & 44.1 & 88.9 \\
\hline Clinical laboratory in hospital ...... & 9.7 & 70.5 \\
\hline Separate nursery for newborn only .... & 64.7 & 95.2 \\
\hline $\begin{array}{l}\text { Graduate nurse on duty at all times } \\
\text { in newborn nursery } \ldots \ldots \ldots \ldots \ldots \ldots \ldots\end{array}$ & 17.6 & 70.5 \\
\hline With Pediatric unit ............... & - & 17.5 \\
\hline Average percentage ................. & 27.0 & 67.0 \\
\hline
\end{tabular}


From 1940 through 1946 there were 1,665 cases of acute poliomyelitis in Kentucky. Of these 188 died. Only 10 hospitals reported that they accept acute poliomyelitis for care; another 17 reported they accept such cases for diagnosis only.

Special hospitals provided more than 25 per cent of care for children in hospitals as expressed in days of child care. They gave 53,673 days of care as against 169,148 estimated days of general hospital care. This estimate made by multiplying child admissions by 7 . The figure for special hospitals does not include the days of care at the Kentucky Training Home for mentally deficient and epileptic children.

There were 61 days of child care per 1000 children in special hospitals in Kentucky compared with 246 in the highest state.

TABLE 16

DAYS OF CARE IN SPECIAL HOSITALS BY TYPE OF HOSPITAL

\begin{tabular}{|c|c|c|}
\hline Type of Hospital & $\begin{array}{l}\text { Number of Hospitals } \\
\text { Admitting Children }\end{array}$ & $\begin{array}{l}\text { Days of } \\
\text { Child Care }\end{array}$ \\
\hline Tuberculosis ............... & 1 & 8,030 \\
\hline Nervous and Mental .......... & - & - \\
\hline 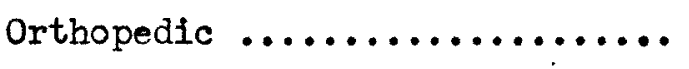 & 2 & 39,212 \\
\hline Eye, Ear, Nose and Throat ...... & 1 & 1,072 \\
\hline Convalescent and Chronic ....... & 1 & 5,359 \\
\hline Contagious Disease .......... & - & - \\
\hline Mentally Deficient and Epileptic & 1 & Not given \\
\hline
\end{tabular}

Six of the general hospitals admitting children also accept children in their outpatient departments. There was only one separate pediatric outpetient clinic in the state located in Louisville. There 
were 12,657 visits by children to outpatient clinics. Of these 8,801 or 70 per cent were in metropolitan counties.

Since this survey was made, Kentucky has inaugurated a State Hospital program under the Federal Hospital Survey anc Construction Act, Public Law 725. To date 15 hospitals have been approved for construction. One of these is a 64 bed tuberculosis hospital which will admit children and 12 are either new or additions to general hospitals. These 12 new hospitals will have about 400 beds. None will have separate pediatric units but all will admit children. 
CHAPTER IV

COMMUNITY HEALTH SERVICES

There are many varieties of child health services available in different communities. Seven types were selected for this study. This is not an exhaustive list. In Kentucky almost all of these services are provided by public agencies. However, considerable financial assistance is given by private groups. The crippled children's program is the best example of community cooperation in this respect. Even though the administering agency is a public one, the medical care is often rendered by private physicians. Table 17 shows the amount of time per month spent by private physicians in child health conferences, school health services, and other medical activities aside from their private practice.

There were 2,699 medical well-child conferences in Kentucky in the year of the study. Of these all but 188 were under public health supervision.

Over half of Kentucky's counties had no well-child conferences during the year. Of the others some had so few meetings that regular supervision was obviously impossible.

The rates for Kentucky are only about one fourth of those of the highest state, and in certain of the rural counties, considerably less than that. Since there was an average of seven children seen at each clinic, there would appear to be no over-crowding. The visits per 
patient were 1.8 indicating that most of the clinics served for examination or immunization only, not for continuing care.

TABLE 17

HOURS FER MONTH OF PRIVATE PHYSICIANS IN COMMUNITY HEAITH SERVICE

\begin{tabular}{c|c|c|c}
\hline Physicians & $\begin{array}{c}\text { Child Health } \\
\text { Conferences }\end{array}$ & $\begin{array}{c}\text { School Health } \\
\text { Conferences }\end{array}$ & $\begin{array}{c}\text { Other Medical } \\
\text { Activities }\end{array}$ \\
\hline $\begin{array}{c}\text { General Practitioners } \\
\text { Participating ...... } \\
\text { All Reporting ...... }\end{array}$ & 16.2 & 7.3 & 17.9 \\
$\begin{array}{c}\text { Pediatricians } \\
\text { Participating ...... }\end{array}$ & 14.7 & 8.8 & 1.3 \\
All Reporting ...... & 1.4 & 1.1 & 33.4 \\
\hline
\end{tabular}

TABLE 18

NUMBER OF CLINICS, FATIENTS, AND VISITS PER 1000 CHILDREN UNDER 5 YFARS OF AGE DURING ONE YEAR BY STATE AND COUNTY GROUP

\begin{tabular}{|c|c|c|c|}
\hline & Sessions & Patients & Visits \\
\hline Highest state ............. & - & 182.0 & 499.0 \\
\hline Average state ............ & - & 76.0 & 233.0 \\
\hline Kentucky ................ & 7.8 & 51.9 & 94.6 \\
\hline 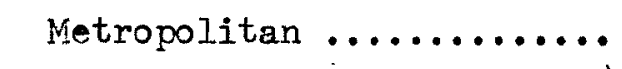 & 15.4 & 119.0 & 245.6 \\
\hline 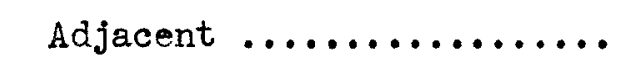 & 2.6 & 24.1 & 34.6 \\
\hline Isolated Semi-rural ....... & 4.5 & 20.7 & 33.1 \\
\hline Isolated Rural ........... & 8.3 & 54.9 & 91.2 \\
\hline
\end{tabular}


Routine immunizations for smallpox and diphtheria, public health nursing follow-up in the home, advice to mothers on formulae, feeding, care and training, generally were given in all clinics. Immunization for whooping cough was given in most rural clinics, but not in the metropolitan. The consultant services of a nutritionist or psychologist were given in most metropolitan clinics. Services by nutritionists were offered in about half the rural clinics, and by psychologists in only a few. In well-child conferences reporting that immunizations were routine procedures, 46.8 of the children were immunized for smallpox, 37.9 for diphtheria, and 32.8 for whooping cough.

There are two mental hygiene clinics offering service to children, one in Louisville and one in Lexington. Out of almost 900,000 children, 552 received mental hygiene service. The number of patients per 1000 children per year was .63 compared with 3.7 for the highest of the eight states. The nurnber of visits per 1000 children was 4.3 compared with 18.0 for the highest of the eight states.

There are 27 clinics offering orthopedic services to physically handicapped children. There are no clinics for rheumatic fever patients or for those with speech difficulties. There are 29 clinics to assist visually handicapped children and 19 counties provide assistance for these children through private physicians. There are 2 hearing clinics and one county provides care for deaf children through private physicians. Public health nursing service is an important part of community health programs. In Kentucky 33 counties have no public health nurse. There are about three times as many children per public health nurse in Kentucky as in the highest state. The number of home visits per year compared with the highest state is shown in Table 19, and about the 
same difference is evident.

TABLE 19

RATES FOR SPECIFIED COMMUNITY HEALTH SERVICE VISITS PER 1000 CHILDREN PER YEAR BY STATE

\begin{tabular}{l|c|c|c|c}
\hline & $\begin{array}{c}\text { Mental } \\
\text { Hygiene }\end{array}$ & $\begin{array}{c}\text { Physically } \\
\text { Handicapped }\end{array}$ & $\begin{array}{c}\text { Dental } \\
\text { Clinic }\end{array}$ & $\begin{array}{c}\text { Public Health } \\
\text { Nursing }\end{array}$ \\
\hline Kentucky ........ & 4.3 & 11.8 & 9.2 & 147.0 \\
Highest State ..... & 18.4 & 31.5 & 103.5 & 486.8 \\
\hline
\end{tabular}

TABLE 20

NUMBER OF CHILDREN PER PUBLIC HEALTH NURSE BY STATE AND COUNTY GROUP

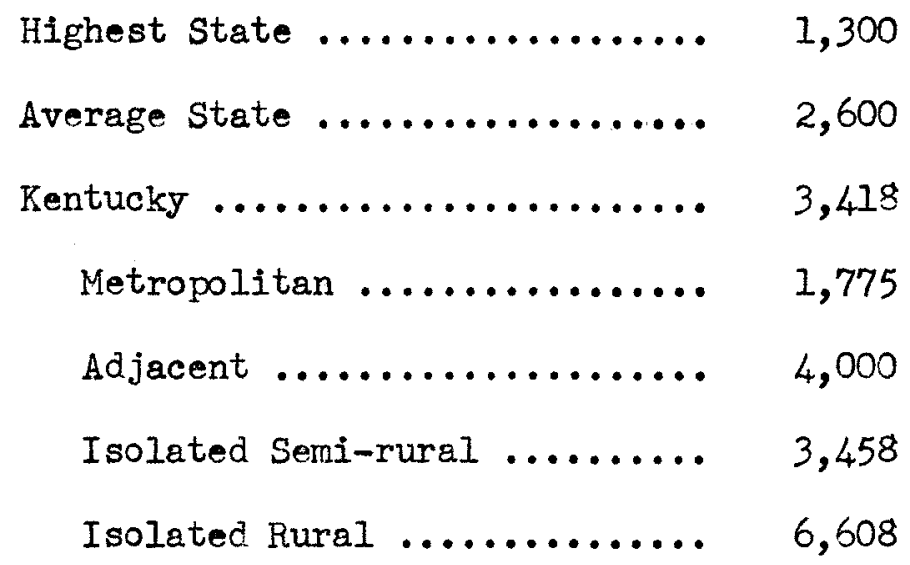

Most of the responsibility for school health programs in Kentucky rests with public health physicians. Forty-four of the counties have no school medical facilities. In the county school systems with medical service, 74 are supervised by public health officers, and 14 others have private physicians. Of the 194 nurses working in schools, 167 are in public health service. Only 2 nurses were employed full time in schools. The others were on a part time basis. 
There are no available estimates of the total number of Immunizations in one year against specific communicable diseases of children. However, there are figures for those immunized by community health agencies in one year.

TABLE 21

IMMUNIZATIONS BY COMMUNTTY HEALTH AGENCIES PER 1000 CHILIREN IN ONE YEAR BY SPECIFIC DISEASES

\section{Immunization Fer} 1000 Children

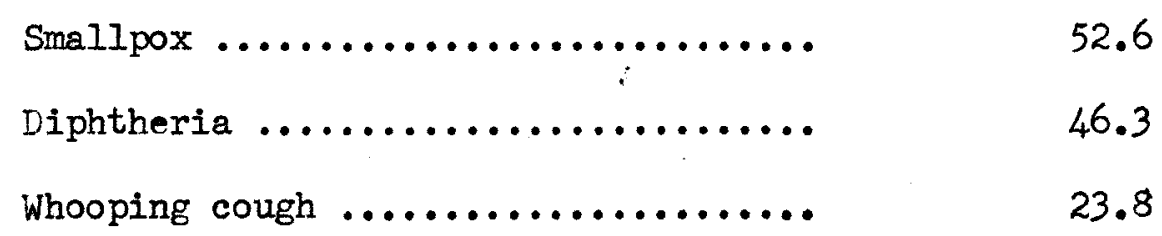

The need of children for dental care is enormous. "Dental examinations of school children in many parts of the country have revealed that 95 per cent are in need of dental service." 1 In this study, a community dental service was defined as one giving dental care other than examination. About one fourth of the dental clinic care of children is given by private agency. The remainder by public health groups. In Table 19 the rate of clinic visits in Kentucky is compared to the highest of the eight states, It is less then one tenth of that state's rates.

The ratio of fillings over extractions is one method of measuring dental service for children. If both services are readily available this ratio may be used as a rough index of the extent of preventive dentistry. This ratio in Kentucky is .96 . In the highest state the ratio is 1.05 . 


\section{CHAPTER V}

TOTAL VOLUME OF MEDICAL CARE

There is no standard available as to the amount of medical care needed by children. What has been done is to assess the total amount of care available and compare it with other states, the highest of which does not claim to have reached the point of covering all needs. A composite picture of the total volume of care received by children on one day has been obtained by adding together the medical care expressed as visits or hospital days rendered to children (1) in the home and office by private physicians; (2) in clinics and conferences; and (3) in hospitals. This gives the total number of children under care in one day and when related to the child population gives an index to the total volume of medical care.

The purpose of this summation is to get a quantitative standard to compare Kentucky with other states and areas within Kentucky. The highest rates in various states do not necessarily represent a standard of adequacy but do give some evidence of what can be done.

If we assume that the total volume of care in the highest state is not more than adequate then Kentucky is quite deficient in caring for her children. The rate for Kentucky is less than 60 per cent of the highest state. The metropolitan counties which have the best rate in the state are only 70 per cent of the rate in the highest state and the rural counties have only 50 per cent of the highest rate. Kentucky is 
particularly deficient in clinic and hospital care. The clinic rate of care being less than 15 per cent of the rate in the highest state; and the hospital rate being less than 40 per cent of the highest state.

TABLE 22

TOTAL VOLUHE OF MEDICAL CARF PER 1000 CHILDREN ON ONE DAY BY STATE AND COUNTY GROUP

\begin{tabular}{l|r|r|r|c}
\hline & \multicolumn{3}{|c|}{ Per 1000 Children Per Day } & \\
\cline { 2 - 5 } & $\begin{array}{c}\text { Total Medical } \\
\text { Care }\end{array}$ & $\begin{array}{c}\text { Visits by } \\
\text { Physician }\end{array}$ & Clinics & Hospitals \\
\cline { 2 - 4 } Highest State .... & 17.1 & 12.4 & 1.3 & 3.4 \\
\cline { 2 - 4 } & 9.7 & 8.3 & .19 & 1.3 \\
Mentucky ........ & 12.3 & 9.9 & .29 & 2.1 \\
\hline
\end{tabular}

of the total number of children under medical care in one day, 17 per cent were receiving health supervision. Most of this group (93 per cent) were under supervision of private practitioners, only 7 per cent by visiting well-child clinics.

Total volume of dental care showed that 1.5 children per 1000 were under dental care in one day. This compares with a rate of 5.3 per 1000 in the highest state.

Kentucky's rate per 1000 children for children under dental care is less than one-third that of the highest state and the rural rate is only about one-fifth of the highest state. Care rendered in dental clinics is negligible. 
TABLE 23

NUMBER OF CHILITEN PER 1000 UNDER DINNTAL CARE ON ONE DAY BY STATE AND COUNTY GROUP

\begin{tabular}{|c|c|c|c|}
\hline & Total & $\begin{array}{l}\text { Private } \\
\text { Practice }\end{array}$ & $\begin{array}{l}\text { Dental } \\
\text { Clinic }\end{array}$ \\
\hline Highest State .............. & 5.3 & 5.2 & 0.15 \\
\hline Kentucky .................. & 1.51 & 1.48 & 0.03 \\
\hline Metropolitan and Adjacent .... & 2.42 & 2.38 & 0.04 \\
\hline Isolated Counties .......... & 1.16 & 1.14 & 0.02 \\
\hline
\end{tabular}




\section{CHAPTER VI}

\section{SUMMARY}

Using population or proximity to densely populated areas as a method of classification, Kentucky's 120 counties are divided as follows: Motropolitan - 5; Adjacent - 15; Isolated semi-rural - 37; Isolated rural - 63. Percentages of Kentucky's children in each group of counties are: Metropolitan, 20 per cent; adjacent, 8 per cent; isolated semi-rural, 38 per cent; and isolated rural, 34 per cent.

Kentucky ranks 18th among the states as to total number of children and 12th as to percentage of children to total population. This comparatively large number of children are cared for by a state whose economic and health facilities are low in comparison with the other states. In one study, Kentucky was ranked 40 th among the states in economic resources, and 46 th as to health and sanitation facilities. The state's per capita income of $\$ 700$ per year was 46 th in the national ranking.

Although Kentucky's infant and maternal death rates have dropped consistently over the period of the last 25 years, nevertheless its rank was still 40 th among the states in infant mortality.

While the percentage of births of children in hospitals increased about 500 per cent from 1935 to 1945, Kentucky still ranked 46th compared to the other states in this respect.

The number of children per physician in Kentucky was about 40 
per cent higher than the average of states with which it was compared and considerably more than twice as high as the state with the best ratio of children to physician. There was a marked diserepancy within the state, the ratio being four times higher in the isolated rural counties than in the metropolitan. These 63 isolated rural counties had almost 1,100 children per physician. The pediatricians and other specialists were concentrated in towns of 10,000 and over.

Sixty-six per cent of the general practitioners had received none or less than one month of training in pediatrics. General practitioners provide more than 80 per cent of children's medical care in Kentucky. Children in metropolitan counties received twice the amount of care, in number of physician's visits, as children in isolated rural counties.

General practitioners saw on the average 20 patients per day of which 6 were children. Pediatricians averaged seeing 22 patients per day.

Most of the child health supervision in Kentucky falls to the general practitioners who are supplying 76 per cent of such care rendered by private physicians.

The number of children per dentist in Kentucky was about 4 times higher than in the highest state. Even the best ratio in metropolitan counties was almost twice that of the highest state; and there were more than 7 times as many children per dentist in the isolated rural counties than the highest state. Nine counties had no dentist at all. The rate of dental care was more than 4 times higher for chilaren in metropolitan than rural counties. 
There were 6.9 hospital beds per 1000 children in Kentucky. This was about one-third of the rate per 1000 children in the highest state. This ratio was more than twice as high in metropolitan than rural counties. A little more than 6 per cent of the beds were set aside exclusively for children, this comperes to about 10 per cent in the highest state.

Hospital admissions per 1000 children was about one-third that of the highest state. In the metropolitan counties this rate was a little more than half the highest state and in the rural counties less than onefourth of that state. Newborn infants received 53 per cent of the total care of children in general hospitals in Kentucky. Kentucky was far below the highest state in the items selected for standards of adequacy in hospital care. Compared to the highest state, Kentucky is low in amount of care rendered children in special hospitals giving only onefourth the number of days' care per 1000 children as that state. More than one-half of Kentucky's counties had no well-child conferences during the year. In several of the counties which did have them, they met so rarely as to be helpful only for examination and imrunization. The number of visits to well-child conferences per 1000 children was less than one-fifth of the rate for the highest state and in some of the rural counties less than one-tenth that rate.

There were only 2 mental hygiene clinics in the state and the rate of visits per 1000 children per year was less than one-fourth that of the highest state.

The rate of service for physically handicapped children was a little more than one-third of that of the highest state.

The number of children per public health nurse was about 3 times 
wigher than that of the highest state; in the isolated rural counties there were 5 times as many children per nurse as in the highest state. In 33 counties there was no public health nurse. In 44 counties there was no school medical service. Only 2 nurses were employed full time by schools. The rate of service in dental clinics was less than onetenth that of the highest state.

By adding the number of visits by private physicians to children, the number of visits to clinics by children, and the days of hospital child care and getting a rate per 1000 children per day we have a standard to measure the amount of care which children are getting. Assuming that children in the state with the highest rating are not getting too much medical care, Kentucky is quite deficient in such care. Kentucky's rate of total care per 1000 children is less than 60 per cent of the highest state. Within the state, children in the metropolitan and adjacent counties receive about 50 per cent more care then children in the rural counties. Of the total number of children under medical are, slightly less than one-fifth were receiving health supervision.

The number of children per 1000 under dental care in one day in Kentucky was about one-third as many as in the highest state. Care given in dental clinics was negligible. 
CHAPTER VII

CONCLUSIONS

Since medical care and facilities are expensive, it is not surprising that Kentucky has less than most other states. Kentucky's relatively low per capita income and deficient economic resources make it difficult to raise the standards of medical care and facilities for her children. The relatively high proportion of children in Kentucky's population is a complication and ggravation of the problem. This is true because, at the present time, children are non-productive and therefore an economic liability. Kentucky, already a poor state, has the added burden of a relatively higher number of non-productive inhabitants. If the children of Kentucky are to have an equal opportunity for medical care with the rest of the children in the United States, federal funds must provide that opportunity. Funds for such a project are simply not available in Kentucky.

Kentucky's needs in order to give more adequate medical care to her children are clearly shown in this report. The first requisite of adequate medical care is plenty of doctors and dentists. Kentucky needs many more doctors and dentists, especially in the rural areas. At least part of this problem is economic, the rural areas are the poorest areas.

The amount of child care which devolves on general practitioners makes it necessary that they receive adequate pediatric training. The fact that, of this group of physicians who render more than 80 per cent 
of child care in Kentucky, more than two-thirds have had none or less than 1 month of hospital pediatric training makes this point clear.

The high incidence of dental care needed speaks for itself of the inadequacy of the present number of dentists to give proper care.

A government program of some sort seems to be the only method of fulfilling these needs. Kentucky's low economic status militates against it in getting men into the medical professions and also in attracting them after they enter the professions. The high expenses entailed in getting a medical or dental education prohibit the relatively poorer people of Kentucky from obtaining such educations and the poor prospect of a return on their investment keep the professional graduates from practicing in Kentuky . These two factors make a program of scholarships and subsidation necessary both to get more medical, dental and nursing personnel and to retain them in the siate after they are trained.

An expansion of public or governmentally financed clinics appears to be one method of filling the eap between no care and private practice which exists at the present time. The clinic program now is less than adequate in the metropolitan areas and non-existent in many of the rural areas. Kentucky's hospitals are less than adequate as to capacity and within the present capacity often inadequate as to facilities. The present state program with federal aid is a start toward a more adequate capacity for hospital care. The deficiency of Kentucky's hospitals in the items selected as criteria of adequate facilities for proper service point up the need for a state licensing law for hospitals.

These facts make a double program necessary for Kentucky. First, present facilities for child care must be used more advantageously in order 
to get the most out of them to neet present needs; and, second, a longrange program to get more better-trained doctors and dentists with adequate clinical and hospital facilities for them to work with must be planned. These prograns will need government financial backing, particularly federal funds, to fill the gap between Kentucky's economic capabilities and the cost of her obvious medical needs. 
APPENDIX 4 
Table 1. Children undor medioal oare on an awerage day, by oounty group in Kentuoky.

\begin{tabular}{|c|c|c|c|c|c|c|}
\hline & \multicolumn{3}{|c|}{ Number } & \multicolumn{3}{|c|}{$\begin{array}{l}\text { Number per day per } \\
100,000 \text { ohildren }\end{array}$} \\
\hline & 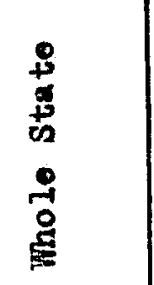 & 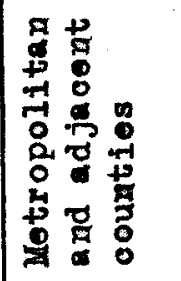 & 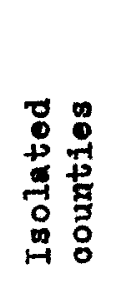 & 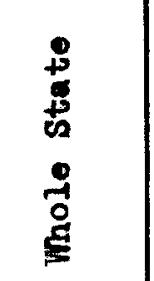 & 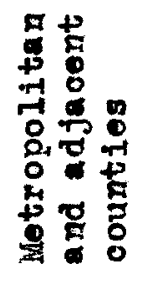 & 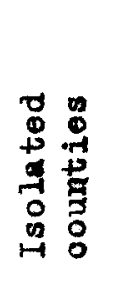 \\
\hline 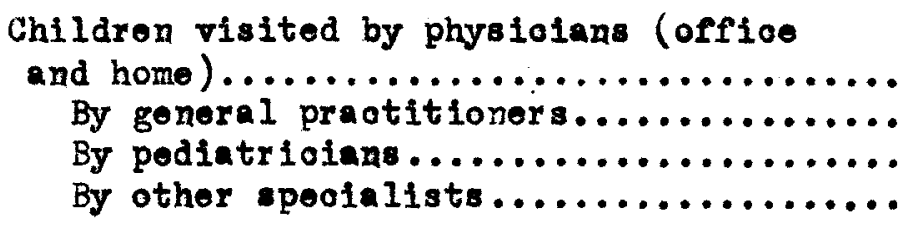 & $\begin{array}{r}7,356 \\
6.277 \\
471 \\
609\end{array}$ & $\begin{array}{r}2,441 \\
1,341 \\
267 \\
333\end{array}$ & $\begin{array}{r}4.915 \\
4.436 \\
204 \\
275\end{array}$ & $\begin{array}{r}834.0 \\
711.7 \\
53.4 \\
68.9\end{array}$ & $\begin{array}{l}992.2 \\
748.3 \\
108.5 \\
135.4\end{array}$ & $\begin{array}{r}772.9 \\
697.6 \\
32.1 \\
43.2\end{array}$ \\
\hline 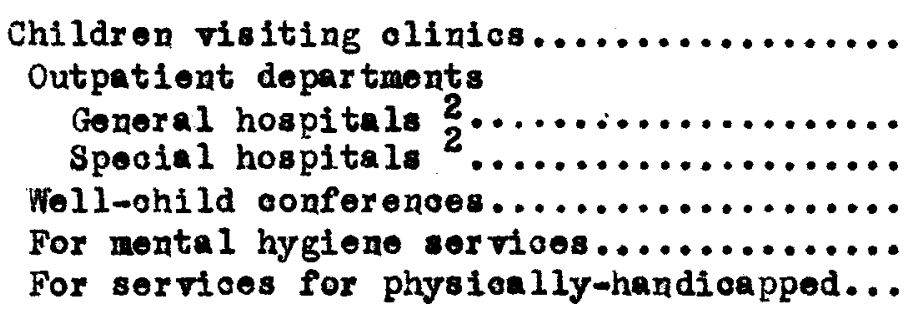 & $\begin{array}{r}165 * \\
34 \\
3 \\
90 \\
10 \\
28\end{array}$ & $\begin{array}{r}72 \\
24 \\
X \\
48 \\
X \\
X\end{array}$ & $\begin{array}{r}62 \\
10 \\
x \\
42 \\
x \\
x\end{array}$ & $\begin{array}{r}18.7 * \\
3.9 \\
0.3 \\
10.2 \\
1.1 \\
3.2\end{array}$ & $\begin{array}{r}29.3 \\
9.8 \\
X \\
19.5 \\
X \\
X\end{array}$ & $\begin{array}{r}8.2 \\
1.6 \\
X \\
6.6 \\
X \\
X\end{array}$ \\
\hline 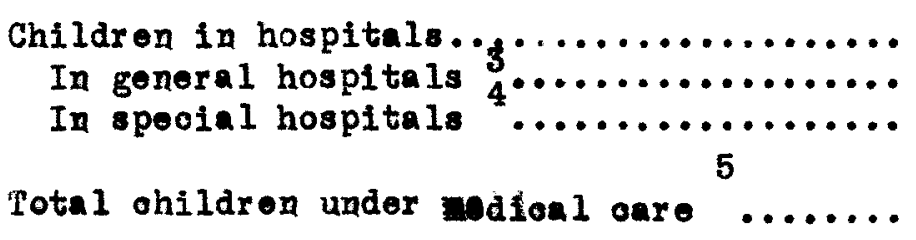 & $\begin{array}{l}1,177 * \\
1,030 \\
147 \\
8,698 *\end{array}$ & $\begin{array}{r}525 \\
525 \\
\times \\
3,038\end{array}$ & $\begin{array}{r}505 \\
505 \\
\times \\
5,472\end{array}$ & $\begin{array}{c}133.5 * \\
116.8 \\
16.7 \\
986.2 *\end{array}$ & $\begin{array}{r}213.4 \\
213.4 \\
X \\
1234.9\end{array}$ & $\begin{array}{r}79.4 \\
79.4 \\
X \\
860.5\end{array}$ \\
\hline
\end{tabular}

1. Items 2 and 4 besed on 1-day rooords; iter 3, on 28-day reoords, items 5-14, on annual figures.

2. If total visits by ohildren not reported, assumed to be equal to visits to pediatrie olinios. If neither figure reported, total visits by ohildren estimated as $10 \%$ of total oPD visits.

3. Days of care estimated as ohildren's admissions times seven, plus days of oare to nowborn.

4. Exclusive of institutions for mentally defioient and epileptio onses.

5. Sum of items 1,5 and 12 .

$X$ Data not given in oounty group because of state-ride olassifioation of servioe.

* Includes items not classified by coupty group. Notes No greater metropolitan oounties in Kentuoky. 

Table 2. Children other than newborn rocoiving modioal aer vice for health supervision on an average
day, by oounty group in Kentuoky.

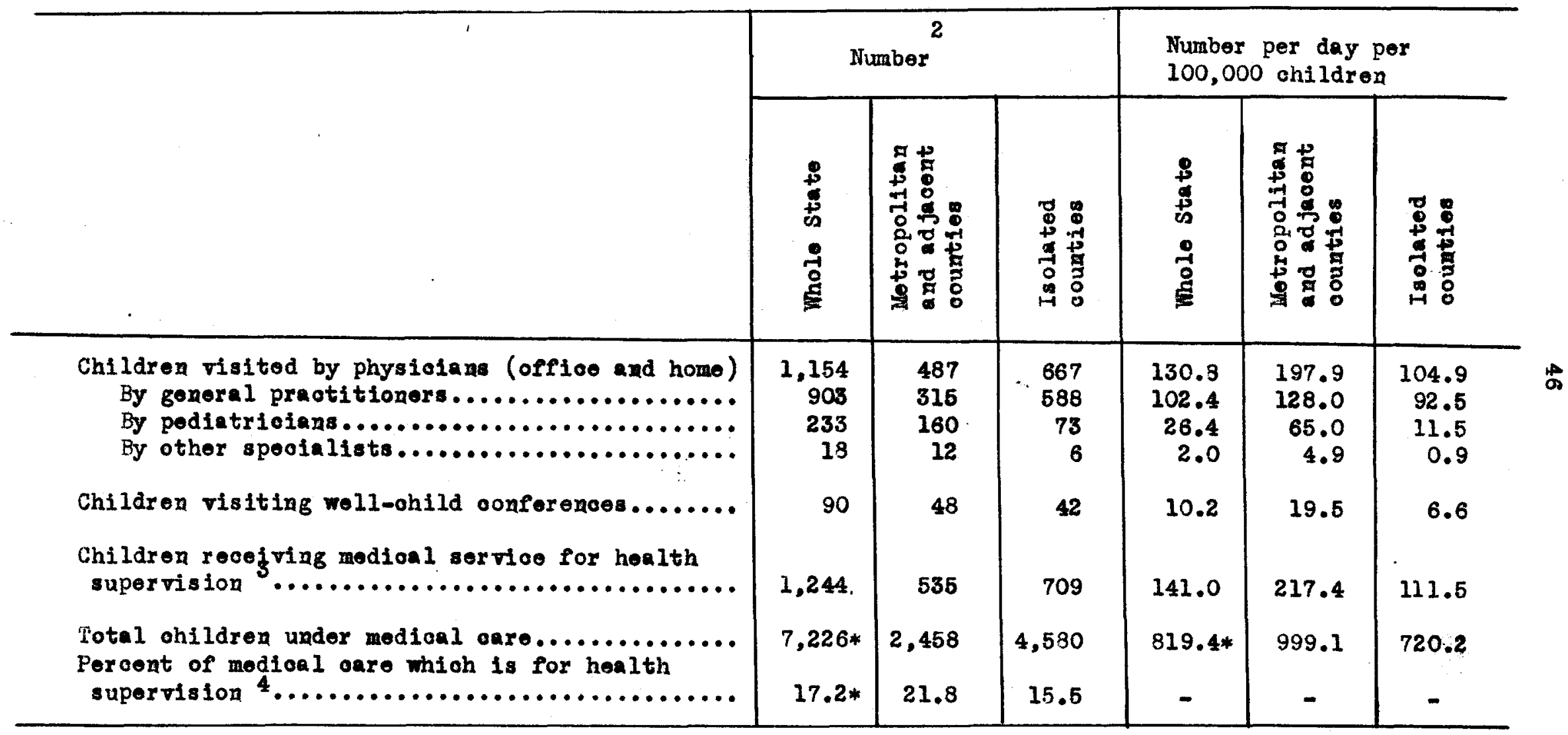

1. Exolusive of health servioes in schools.

2. Items 2 and 4 based on l-day records of physiofans; iten 3 , on 28-day records; item 5 on annual figure

3. Sum of itoms 1 and 5 .

4. Line 6 divided by line 7 .

* Inoludes items not classified by oounty group (as on Table 1). 
Table 3. Infants and preschool children receiving medioal service for health supervision on an average day, by oounty group in Kentucky.

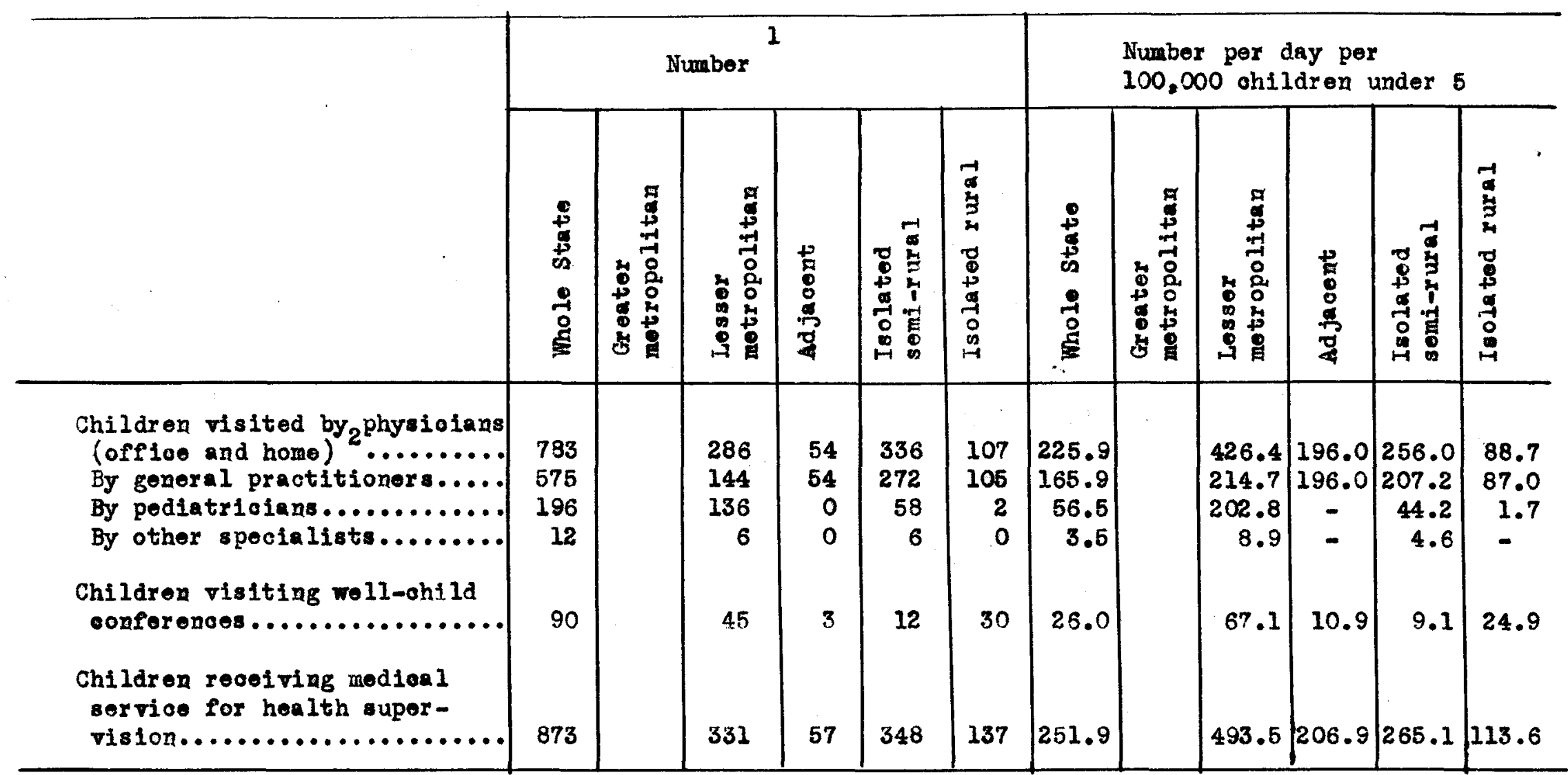

1. Items 2 and 4 based on 1-day records; item 3, on 28-day reoords; itom 5 on annual figures.

2. Visits to ohildx on of ages 1 month -5 years. 
Tablo 4. Children under dental oare on an average day, by county group in Kentuoky

\begin{tabular}{|c|c|c|c|c|c|c|}
\hline \multirow[t]{2}{*}{. } & \multicolumn{3}{|c|}{ Number ${ }^{1}$} & \multicolumn{3}{|c|}{$\begin{array}{l}\text { Number per day por } \\
100,000 \text { ohildren }\end{array}$} \\
\hline & 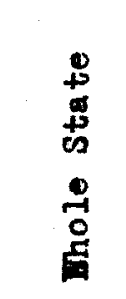 & 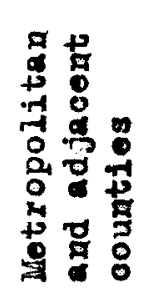 & 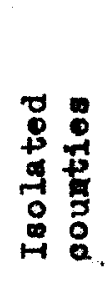 & $\begin{array}{l}0 \\
+ \\
0 \\
0 \\
0 \\
0 \\
0 \\
0 \\
0\end{array}$ & 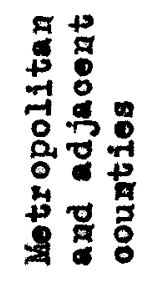 & 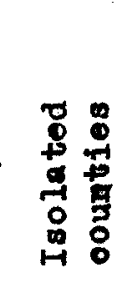 \\
\hline 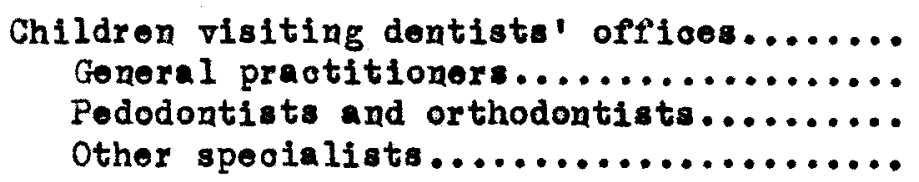 & 1,311 & 585 & 726 & 148.7 & 237.8 & 114.2 \\
\hline $\begin{array}{l}\text { Children visiting dental olinios for } \\
\text { services other than examinations.......... } \\
\text { Total children reoeiving dental care....... }\end{array}$ & $\begin{array}{r}22 \\
1,333\end{array}$ & $\begin{array}{r}9 \\
594\end{array}$ & $\begin{array}{r}13 \\
739\end{array}$ & $\begin{array}{r}2.5 \\
-\quad 151.2\end{array}$ & $\begin{array}{r}3.7 \\
241.5\end{array}$ & $\begin{array}{r}2.0 \\
116.2\end{array}$ \\
\hline
\end{tabular}

1. Items 1-4 are based on 1-day records of dentists; item 5, on annual figures. 
Table 5. Physioians and dentists in prirate practioe in Kentucky by type of speoialty, oertification by Amerioan Spoolalty Boards and oounty group, as of September, 1946.

\begin{tabular}{|c|c|c|c|c|c|c|c|c|}
\hline & \multicolumn{7}{|c|}{ Number of professional porsonnel in - } & \multirow[b]{2}{*}{$\begin{array}{l}0 \\
0 \\
0 \\
0\end{array}$} \\
\hline o & 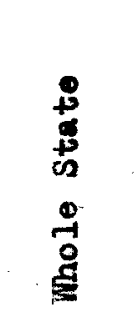 & 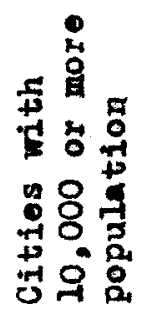 & 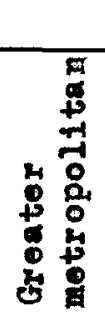 & 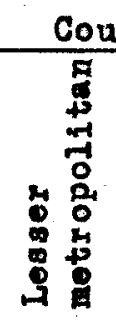 & $\begin{array}{l}+ \\
\stackrel{8}{8} \\
8 \\
\\
\end{array}$ & 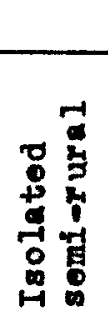 & 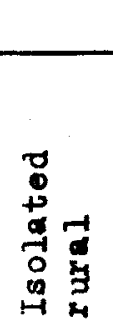 & \\
\hline 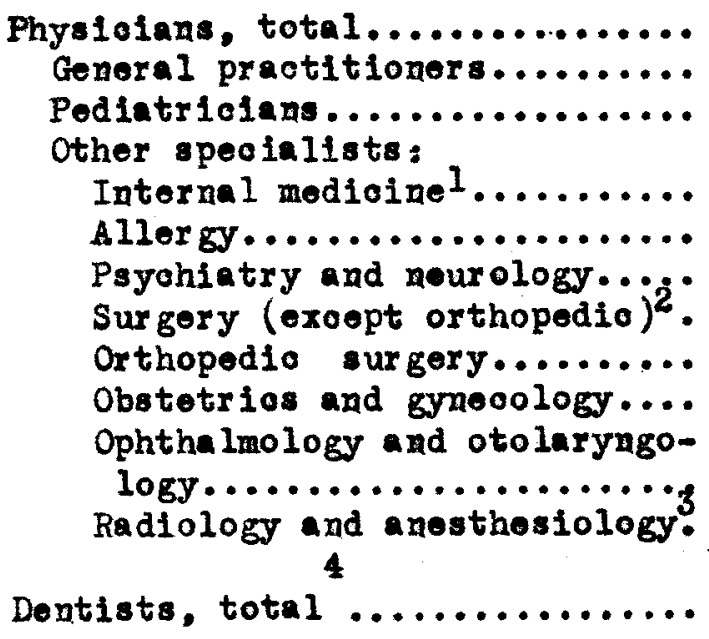 & $\begin{array}{r}1,751 \\
1,206 \\
31 \\
98 \\
2 \\
11 \\
201 \\
11 \\
53 \\
107 \\
31 \\
698\end{array}$ & $\begin{array}{r}881 \\
429 \\
27 \\
90 \\
2 \\
11 \\
160 \\
11 \\
46 \\
75 \\
30 \\
373\end{array}$ & & $\begin{array}{r}657 \\
335 \\
16 \\
66 \\
2 \\
9 \\
110 \\
9 \\
37 \\
49 \\
24 \\
288\end{array}$ & $\begin{array}{r}117 \\
102 \\
0 \\
3 \\
0 \\
0 \\
8 \\
0 \\
1 \\
3 \\
0 \\
46\end{array}$ & $\begin{array}{r}682 \\
484 \\
14 \\
28 \\
0 \\
2 \\
80 \\
2 \\
13 \\
\\
62 \\
7 \\
257\end{array}$ & $\begin{array}{r}296 \\
285 \\
1 \\
1 \\
0 \\
0 \\
3 \\
0 \\
2 \\
3 \\
0 \\
107\end{array}$ & $\begin{array}{r}- \\
- \\
17 \\
26 \\
1 \\
4 \\
41 \\
6 \\
10 \\
\\
33 \\
13 \\
\end{array}$ \\
\hline
\end{tabular}

1. Inoludes dermatology, syphilology, oardiovasoular disease, gastroenterology and tuberculosis.

2. Inoludes urology, plastio surgery, nourologioal surgery and prootology.

3. Inoludes clinical pathology and baotoriology.

4. Breakdown by speoialty not given sinoe 260 out of 698 dentists in Kontuoky did not report whether they linited their practioe to ono specialty. 
Table 6. Physioians and dentists in private practice, by type of specialty in each county and cach ofty with 10,000 or more population in Kentuoky.

\begin{tabular}{|c|c|c|c|c|c|c|c|c|c|c|c|c|}
\hline \multirow[b]{3}{*}{$\begin{array}{l}\text { County } \\
\text { and oity }\end{array}$} & \multicolumn{11}{|c|}{ Number of physiojans } & \multirow{3}{*}{ 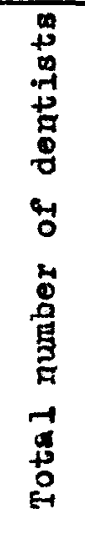 } \\
\hline & \multirow[b]{2}{*}{\begin{tabular}{l}
$\vec{a}$ \\
\multirow{0}{0}{} \\
0 \\
$\xi$
\end{tabular}} & \multirow[b]{2}{*}{ 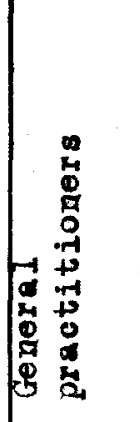 } & \multirow[b]{2}{*}{ 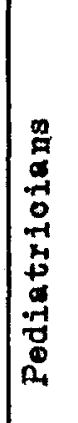 } & \multicolumn{8}{|c|}{ Other speoialis ts } & \\
\hline & & & & 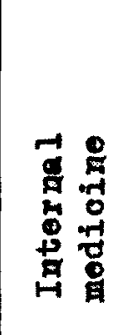 & $\begin{array}{l}80 \\
0 \\
0 \\
0 \\
-1 \\
-4\end{array}$ & 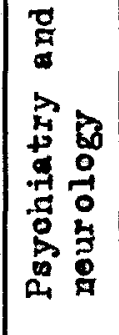 & 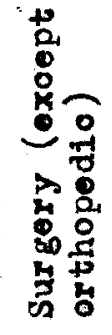 & 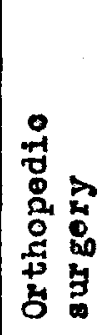 & 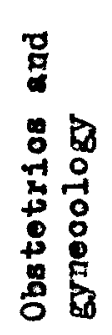 & 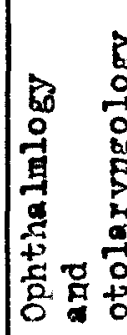 & 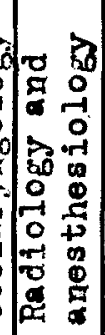 & \\
\hline 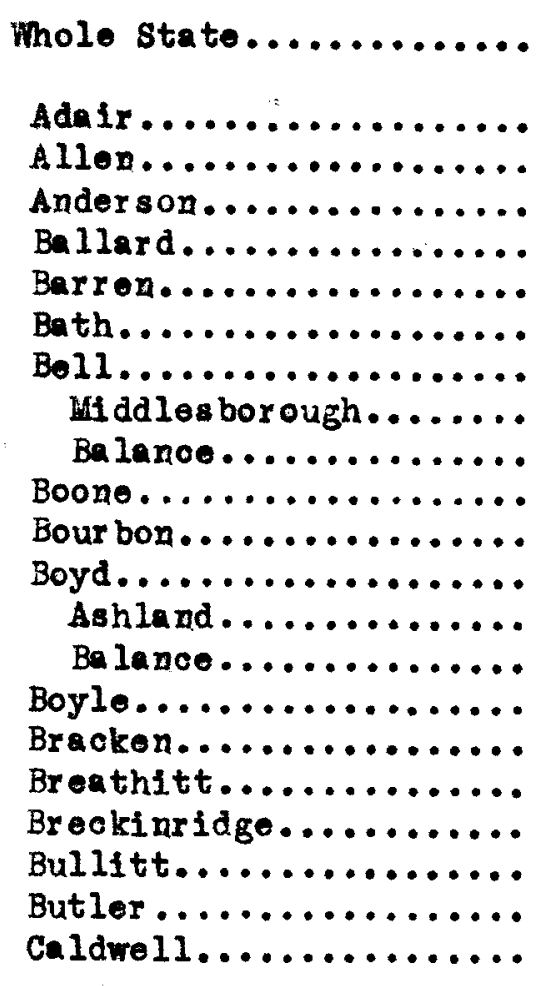 & $\begin{array}{r}1.751 \\
6 \\
4 \\
6 \\
8 \\
18 \\
6 \\
26 \\
18 \\
8 \\
7 \\
14 \\
39 \\
38 \\
1 \\
17 \\
9 \\
1 \\
5 \\
1 \\
2 \\
8\end{array}$ & \begin{tabular}{|r|r}
1,206 \\
6 \\
4 \\
5 \\
8 \\
11 \\
6 \\
23 \\
$\frac{15}{8}$ \\
7 \\
13 \\
25 \\
24 \\
1 \\
15 \\
8 \\
1 \\
5 \\
1 \\
2 \\
5
\end{tabular} & \begin{tabular}{|l|l}
31 \\
$\frac{1}{1}$ \\
\\
\\
\end{tabular} & $\frac{1}{1}$ & 2 & 11 & $\begin{array}{l}5 \\
\frac{1}{1}\end{array}$ & 11 & $\frac{2}{2}$ & $\begin{array}{l}2 \\
\frac{1}{I} \\
1 \\
\frac{4}{4} \\
1 \\
1\end{array}$ & $\frac{I}{I}$ & $\begin{array}{r}698 \\
2 \\
3 \\
2 \\
1 \\
7 \\
1 \\
10 \\
3 \\
7 \\
2 \\
5 \\
19 \\
18 \\
1 \\
6 \\
2 \\
1 \\
2 \\
2 \\
2 \\
3\end{array}$ \\
\hline
\end{tabular}


Table 6. (oontid) Physicians and dentists in rpivate practice, by typo of specialty in each oounty and each city with 10,000 or more population in Kentuoky.

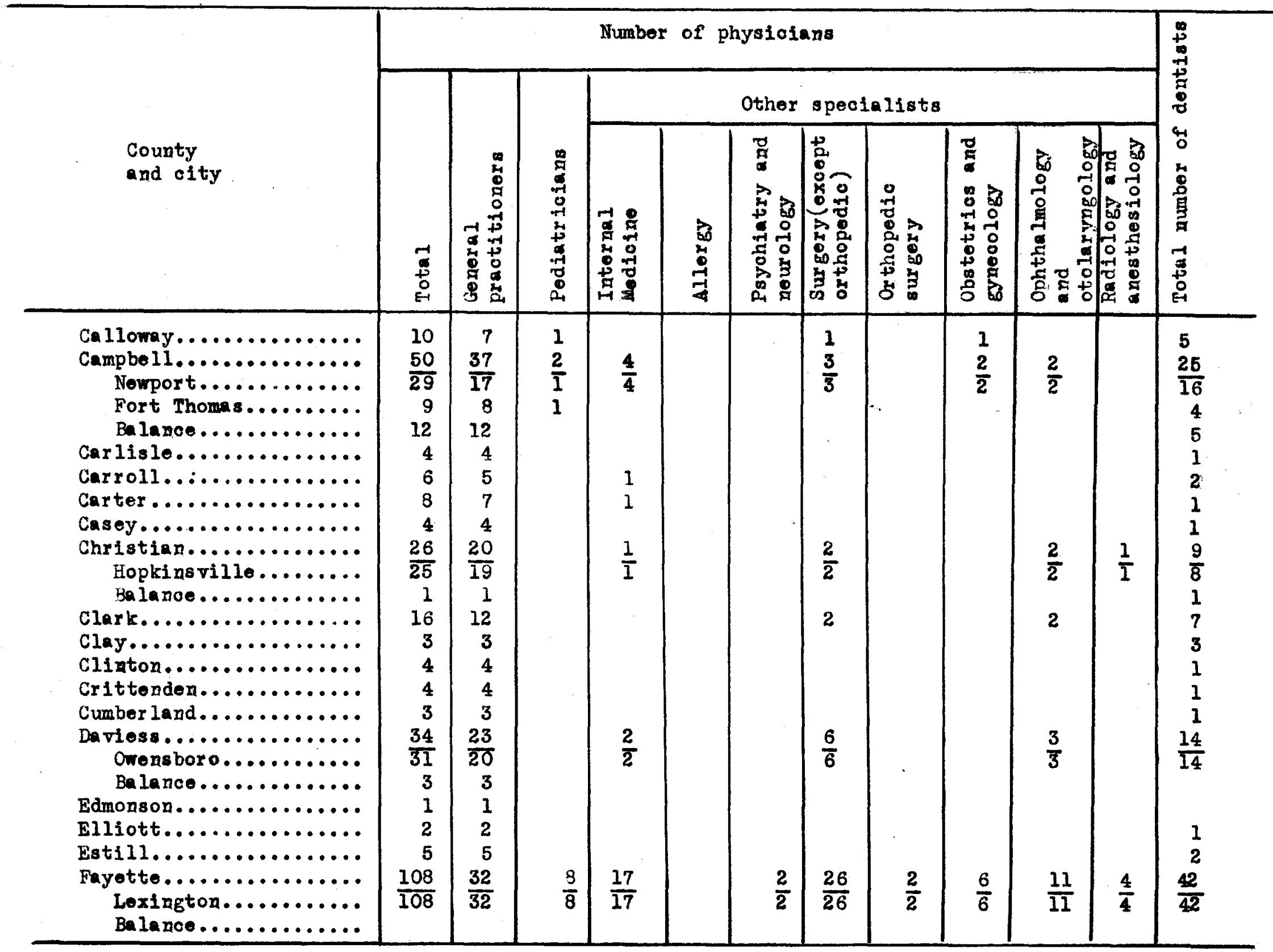



Tablo 6 (oont'd). Physioians and dontists in private practioe, by type of spooiality in oach oounty
and oach olty with 10,000 or more population in Kontuoky

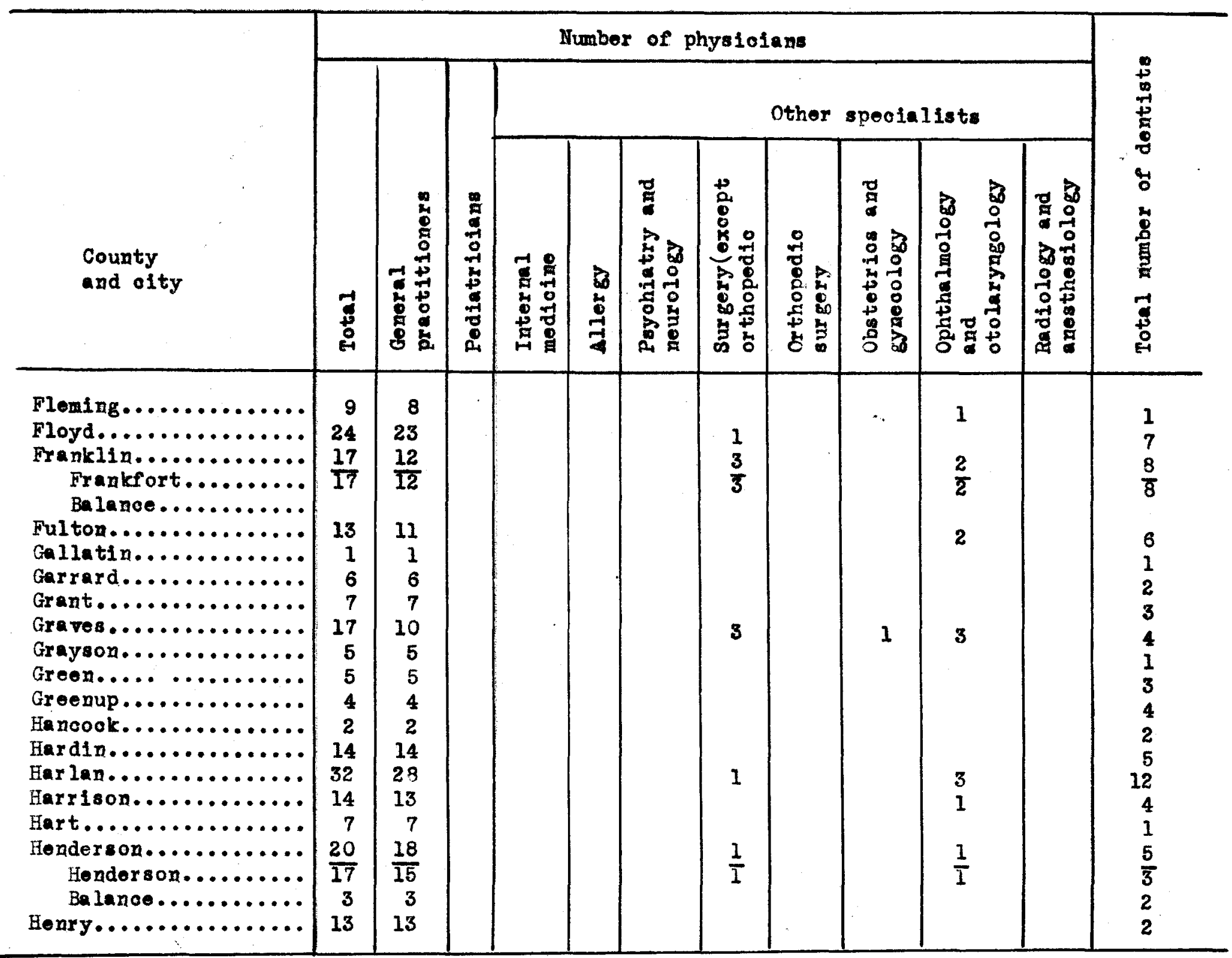


Pabio 6 (cont'd). Physioians and dentists in privato practioe, by type of spooialty in each oounty and oity with 10,000 or more population in Kentucky.

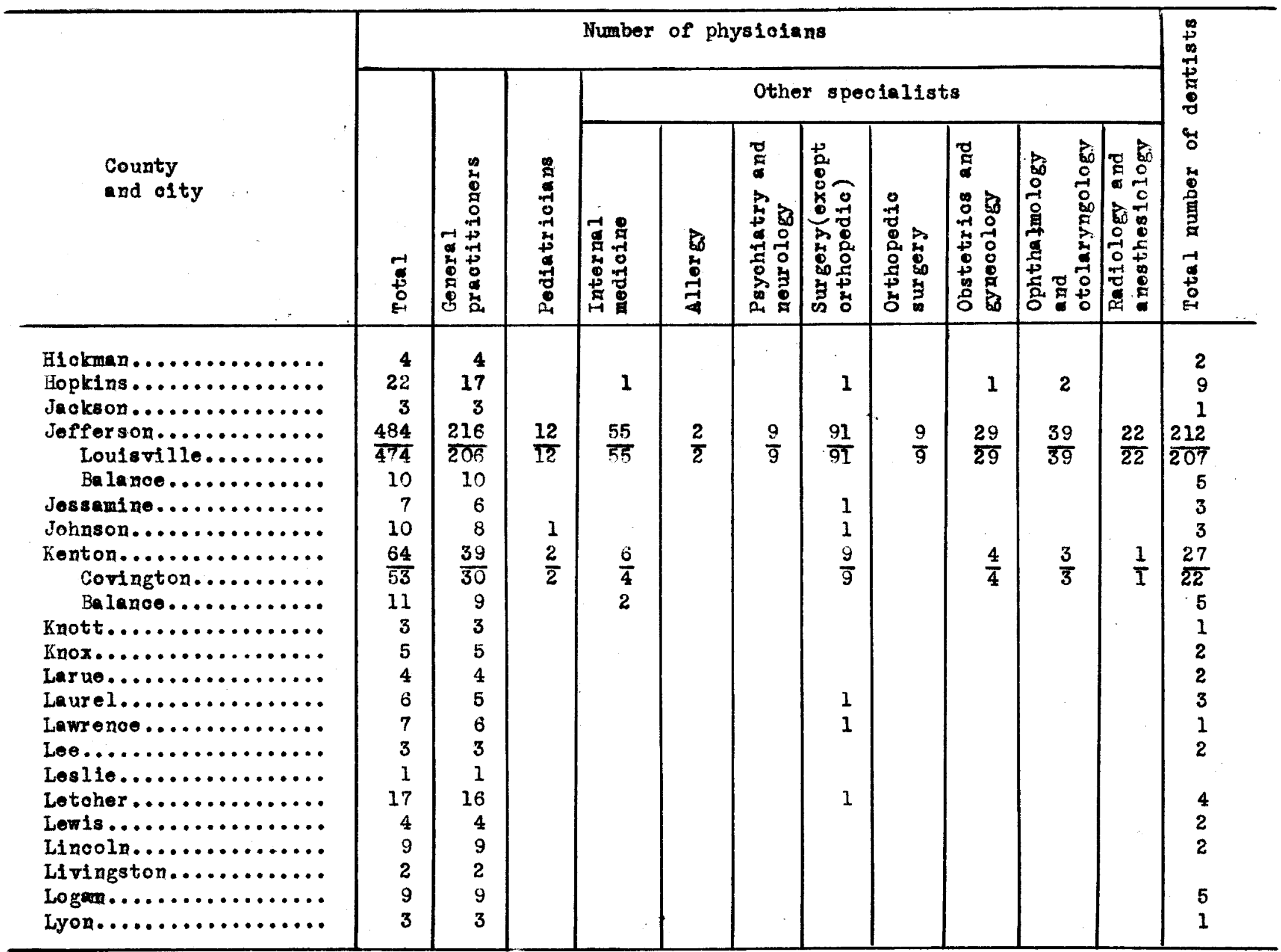


Table 6 (oont'd). Physioians and dentists in private practioe, by type of speoialty in oach oounty and oa oh oity with 10,000 or more population in Kentuoky.

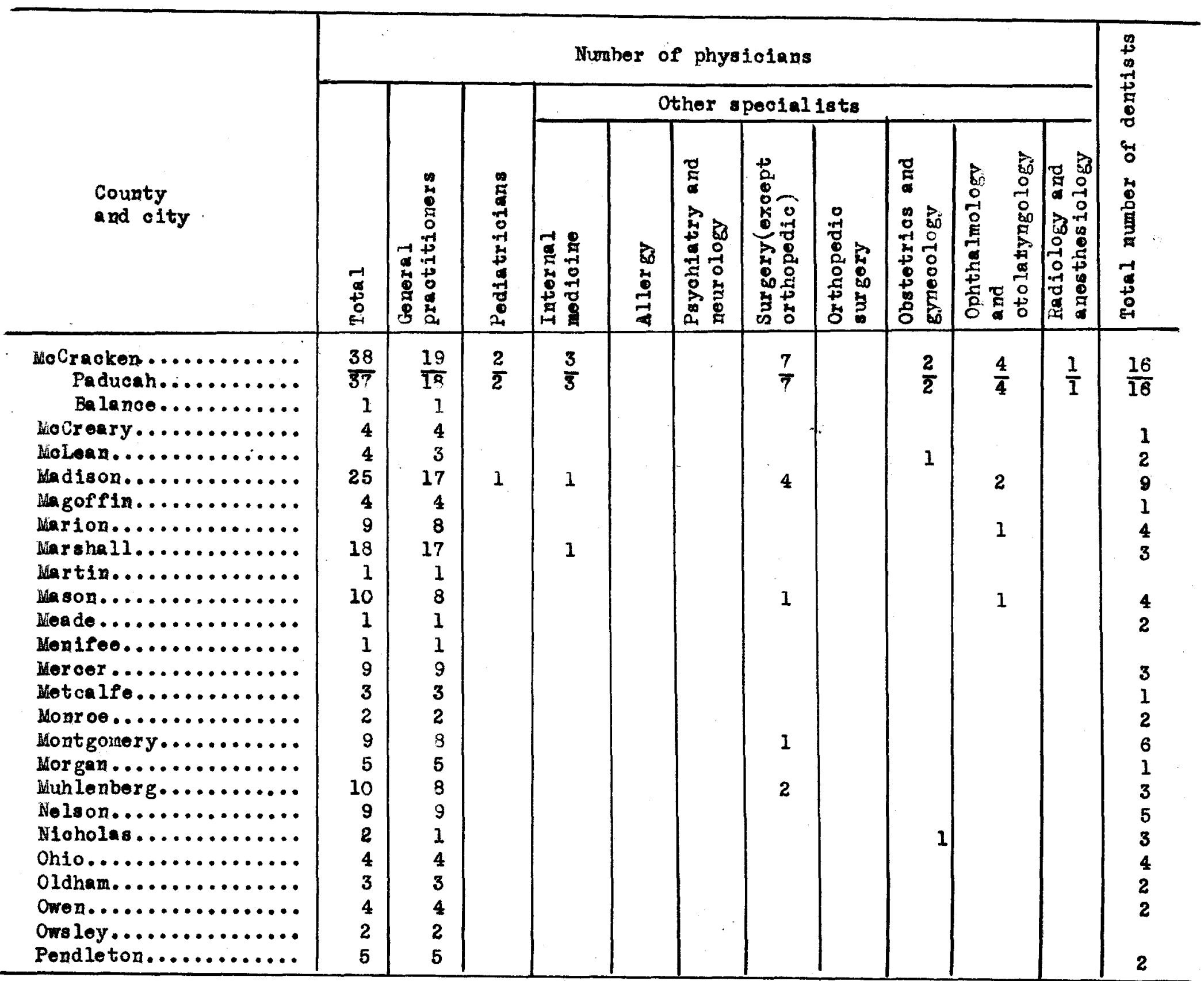


Table 6 (cont'd). Physioians and dentists in private practice, by type of speoialty ili oach county and oach oity with 10,000 or more population in Kentucky.

\begin{tabular}{|c|c|c|c|c|c|c|c|c|c|c|c|c|}
\hline \multirow[b]{3}{*}{$\begin{array}{l}\text { County } \\
\text { and city }\end{array}$} & \multicolumn{11}{|c|}{ Number of physicians } & \multirow{3}{*}{ 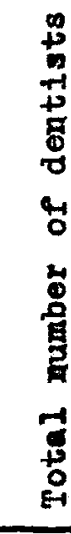 } \\
\hline & \multirow[b]{2}{*}{ 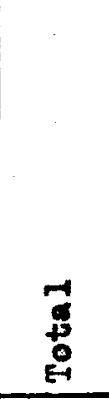 } & \multirow[b]{2}{*}{ 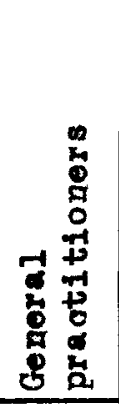 } & \multirow[b]{2}{*}{ 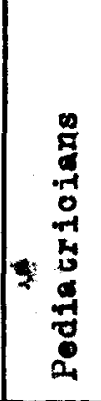 } & \multicolumn{8}{|c|}{ Other spooinlists } & \\
\hline & & & & 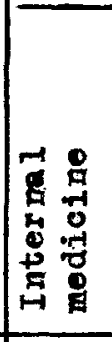 & 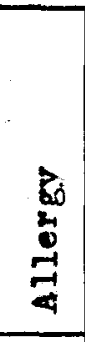 & 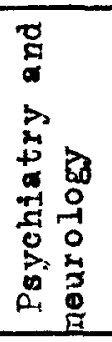 & 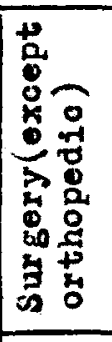 & 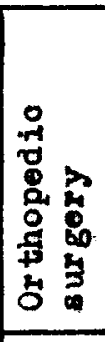 & 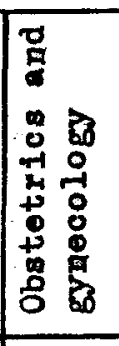 & 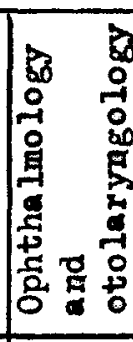 & 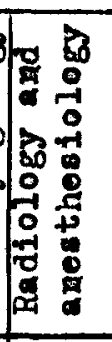 & \\
\hline 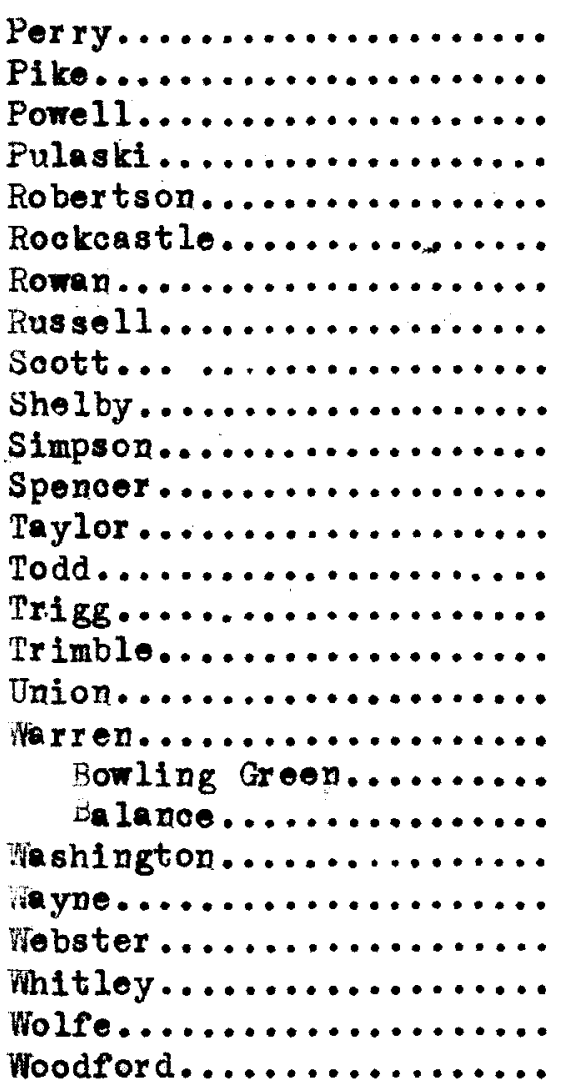 & $\begin{array}{r}23 \\
25 \\
2 \\
21 \\
2 \\
6 \\
3 \\
4 \\
14 \\
9 \\
8 \\
4 \\
7 \\
4 \\
5 \\
2 \\
11 \\
27 \\
25 \\
2 \\
4 \\
4 \\
11 \\
20 \\
2 \\
9\end{array}$ & $\begin{array}{r}18 \\
21 \\
2 \\
16 \\
2 \\
5 \\
3 \\
4 \\
12 \\
9 \\
7 \\
4 \\
7 \\
4 \\
5 \\
2 \\
10 \\
15 \\
13 \\
2 \\
4 \\
4 \\
11 \\
16 \\
2 \\
9\end{array}$ & 1 & $\frac{3}{3}$ & 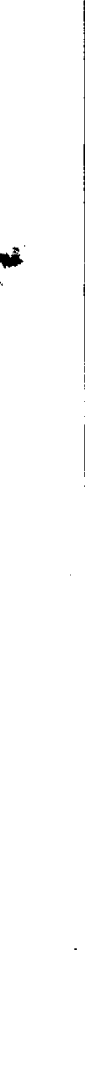 & & $\begin{array}{l}2 \\
2 \\
2\end{array}$ & & $\frac{1}{I}$ & $\begin{array}{l}2 \\
1 \\
1 \\
1 \\
1 \\
1\end{array}$ & 1 & $\begin{array}{r}6 \\
9 \\
1 \\
8 \\
1 \\
3 \\
2 \\
2 \\
4 \\
2 \\
4 \\
3 \\
1 \\
6 \\
13 \\
12 \\
1 \\
4 \\
1 \\
2 \\
7 \\
2 \\
2\end{array}$ \\
\hline
\end{tabular}


Table 7. Availability of physicians and dentists in nrivate practioe, by county group in Kentuckg.

\begin{tabular}{|c|c|c|c|c|c|c|c|c|}
\hline \multirow[b]{2}{*}{ County group } & \multicolumn{4}{|c|}{ Physioiano } & \multicolumn{4}{|c|}{ Dentists } \\
\hline & $\begin{array}{l}\vec{a} \\
\stackrel{a}{+} \\
\stackrel{0}{0}\end{array}$ & 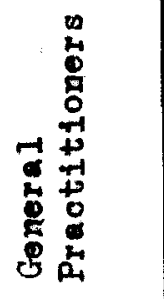 & 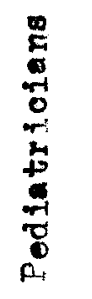 & 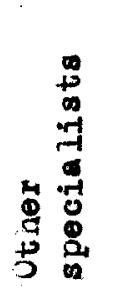 & $\begin{array}{l}\overrightarrow{1} \\
\stackrel{5}{+} \\
\stackrel{0}{0} \\
\stackrel{0}{*}\end{array}$ & 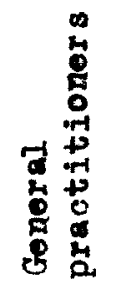 & 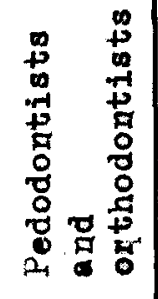 & 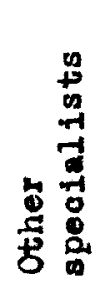 \\
\hline & \multicolumn{8}{|c|}{ Number. } \\
\hline 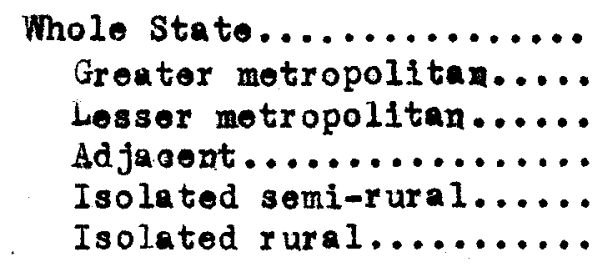 & $\begin{array}{r}1,751 \\
657 \\
117 \\
682 \\
295\end{array}$ & $\begin{array}{r}1,206 \\
335 \\
102 \\
484 \\
285\end{array}$ & $\begin{array}{r}31 \\
16 \\
0 \\
14 \\
1\end{array}$ & $\begin{array}{r}514 \\
306 \\
15 \\
184 \\
9\end{array}$ & $\begin{array}{r}698 \\
288 \\
46 \\
257 \\
107\end{array}$ & & & - \\
\hline & \multicolumn{8}{|c|}{ Number per 100,000 children } \\
\hline $\begin{array}{l}\text { Whole state................. } \\
\text { Greater metropolitan..... } \\
\text { Lesser motropolitan..... } \\
\text { Adjaoont................. } \\
\text { Isolated semi-rural...... } \\
\text { Isolated rural.......... }\end{array}$ & $\begin{array}{r}198.5 \\
378.0 \\
162.1 \\
205.7 \\
96.9\end{array}$ & $\begin{array}{r}136.7 \\
192.8 \\
141.3 \\
146.0 \\
93.6\end{array}$ & $\begin{array}{l}3.5 \\
9.2 \\
- \\
4.2 \\
0.3\end{array}$ & $\begin{array}{r}58.3 \\
176.0 \\
20.8 \\
55.5 \\
3.0\end{array}$ & $\begin{array}{r}79.1 \\
165.7 \\
63.7 \\
77.5 \\
35.2\end{array}$ & & & \\
\hline
\end{tabular}


Table 8. VIsits per day for siok and well children by general modioal praotitioners, pediatrioians and other specialists, by oounty group in Kentuoky.

\begin{tabular}{|c|c|c|c|c|c|c|c|c|c|}
\hline \multirow[b]{2}{*}{ County group } & \multicolumn{4}{|c|}{$\begin{array}{l}\text { Number of visits by } \\
\text { speoified physiolans }\end{array}$} & \multicolumn{4}{|c|}{$\begin{array}{l}\text { Percent of all visits made } \\
\text { by specified physioians }\end{array}$} & \multirow[b]{2}{*}{ 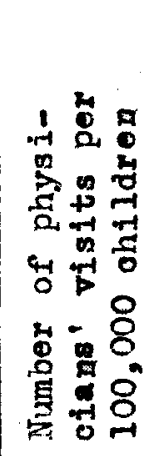 } \\
\hline & 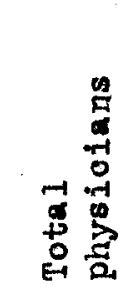 & 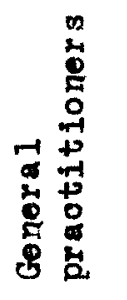 & 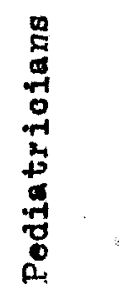 & 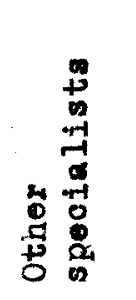 & 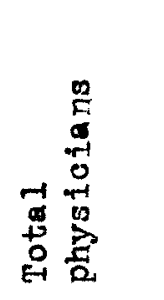 & 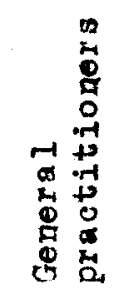 & 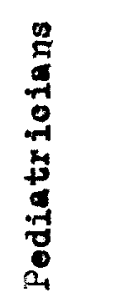 & 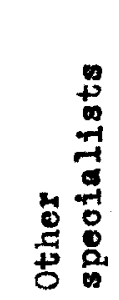 & \\
\hline \multirow{3}{*}{$\begin{array}{l}\text { Wholo stato..... } \\
\text { Lesser metro- } \\
\text { politan........ } \\
\text { Adjaoont....... } \\
\text { Isolated soini- } \\
\text { rural......... } \\
\text { Isolated rural... }\end{array}$} & \multicolumn{8}{|c|}{ Total visits for siok and well ohildren } & $\Rightarrow$ \\
\hline & $\begin{array}{r}8,711 \\
2,424 \\
623 \\
3,537 \\
2,127 \\
\end{array}$ & $\begin{array}{r}7.124 \\
1,549 \\
599 \\
2,387 \\
2,089\end{array}$ & $\begin{array}{r}628 \\
338 \\
0 \\
272 \\
18\end{array}$ & $\begin{array}{r}959 \\
537 \\
24 \\
378 \\
20 \\
\end{array}$ & $\begin{array}{l}100.0 \\
100.0 \\
100.0 \\
100.0 \\
100.0 \\
\end{array}$ & $\begin{array}{l}81.8 \\
63.9 \\
96.1 \\
81.6 \\
98.3 \\
\end{array}$ & $\begin{array}{c}7.2 \\
13.9 \\
- \\
7.7 \\
0.8 \\
\end{array}$ & $\begin{array}{r}11.0 \\
22.2 \\
3.9 \\
10.7 \\
0.9 \\
\end{array}$ & $\begin{array}{r}987.7 \\
1,394.5 \\
863.0 \\
1,066.8 \\
698.9 \\
\end{array}$ \\
\hline & & & & V181ts & or siok & aildren & & & \\
\hline \multirow[t]{2}{*}{$\begin{array}{l}\text { Wholo state...... } \\
\text { Lesser metro- } \\
\text { politan....... } \\
\text { Adjacent........ } \\
\text { Isolated semi- } \\
\text { rural......... } \\
\text { Isolated rural.. }\end{array}$} & $\begin{array}{r}6,903 \\
1,772 \\
519 \\
2,279 \\
1,883\end{array}$ & $\begin{array}{r}5,744 \\
1,196 \\
495 \\
\\
2,206 \\
1,847\end{array}$ & $\begin{array}{r}318 \\
148 \\
0 \\
154 \\
16\end{array}$ & $\begin{array}{r}841 \\
428 \\
24 \\
369 \\
20\end{array}$ & $\begin{array}{l}100.0 \\
100.0 \\
100.0 \\
100.0 \\
100.0\end{array}$ & $\begin{array}{l}83.2 \\
\\
67.4 \\
95.4 \\
\\
80.9 \\
98.1 \\
\end{array}$ & $\begin{array}{l}4.6 \\
8.4 \\
- \\
5.6 \\
0.8 \\
\end{array}$ & $\begin{array}{r}12.2 \\
24.2 \\
4.6 \\
13.5 \\
1.1 \\
\end{array}$ & $\begin{array}{r}782.7 \\
1,019.4 \\
718.9 \\
823.1 \\
618.7\end{array}$ \\
\hline & \multicolumn{9}{|c|}{ Visits for well childron } \\
\hline $\begin{array}{l}\text { thole state..... } \\
\text { Lesser metro- } \\
\text { politan........ } \\
\text { Adjacent........ } \\
\text { Isolated semi- } \\
\text { rural......... } \\
\text { Isolated rural... }\end{array}$ & $\begin{array}{r}1,808 \\
652 \\
104 \\
808 \\
244\end{array}$ & $\begin{array}{l}353 \\
104 \\
681 \\
242\end{array}$ & $\begin{array}{r}310 \\
190 \\
0 \\
118 \\
2\end{array}$ & $\begin{array}{r}118 \\
109 \\
0\end{array}$ & $\begin{array}{l}100.0 \\
100.0 \\
100.0\end{array}$ & $\begin{array}{r}76.4 \\
54.2 \\
100.0 \\
84.3 \\
99.2\end{array}$ & $\begin{array}{l}17.1 \\
29.1 \\
- \\
14.6 \\
0.8\end{array}$ & $\begin{array}{l}6.5 \\
16.7 \\
- \\
1.1\end{array}$ & $\begin{array}{r}205.0 \\
375.1 \\
144.1 \\
243.7 \\
80.2\end{array}$ \\
\hline
\end{tabular}


Table 9. Children's risits per day by general dental practitioners, pedodontists, orthodontists and other spocialists, by oounty group in Kontuoky.

\begin{tabular}{|c|c|c|c|c|c|c|c|c|c|}
\hline \multirow[b]{2}{*}{ County group } & \multicolumn{4}{|c|}{$\begin{array}{l}\text { Number of visits by } \\
\text { spocified dentists }\end{array}$} & \multicolumn{4}{|c|}{$\begin{array}{l}\text { Peroent of all visits made } \\
\text { by specified dentists }\end{array}$} & \multirow{2}{*}{ 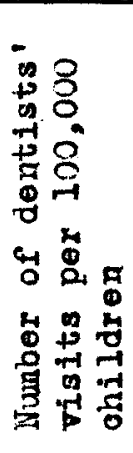 } \\
\hline & 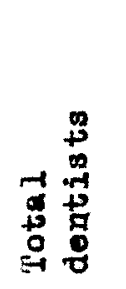 & 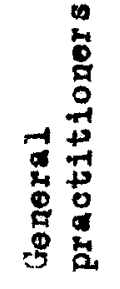 & 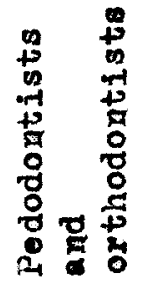 & 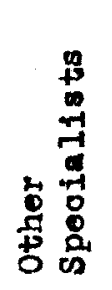 & 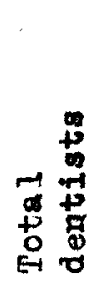 & 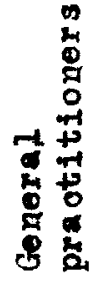 & 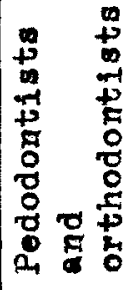 & 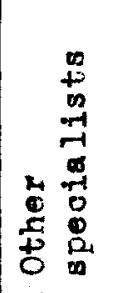 & \\
\hline $\begin{array}{l}\text { Molo State............ } \\
\text { Greater metropolitan... } \\
\text { Lesser metropolitan.... } \\
\text { Adjacent.............. } \\
\text { Isolated semi-rural.... } \\
\text { Isolated rural......... }\end{array}$ & $\begin{array}{r}1,311 \\
480 \\
105 \\
524 \\
202\end{array}$ & & & & & & & & $\begin{array}{r}148.7 \\
276.1 \\
145.5 \\
158.0 \\
66.4\end{array}$ \\
\hline
\end{tabular}


Table 12. Age, race and sex of physioiajs in private praotice, by county gxoup in Kentucky.

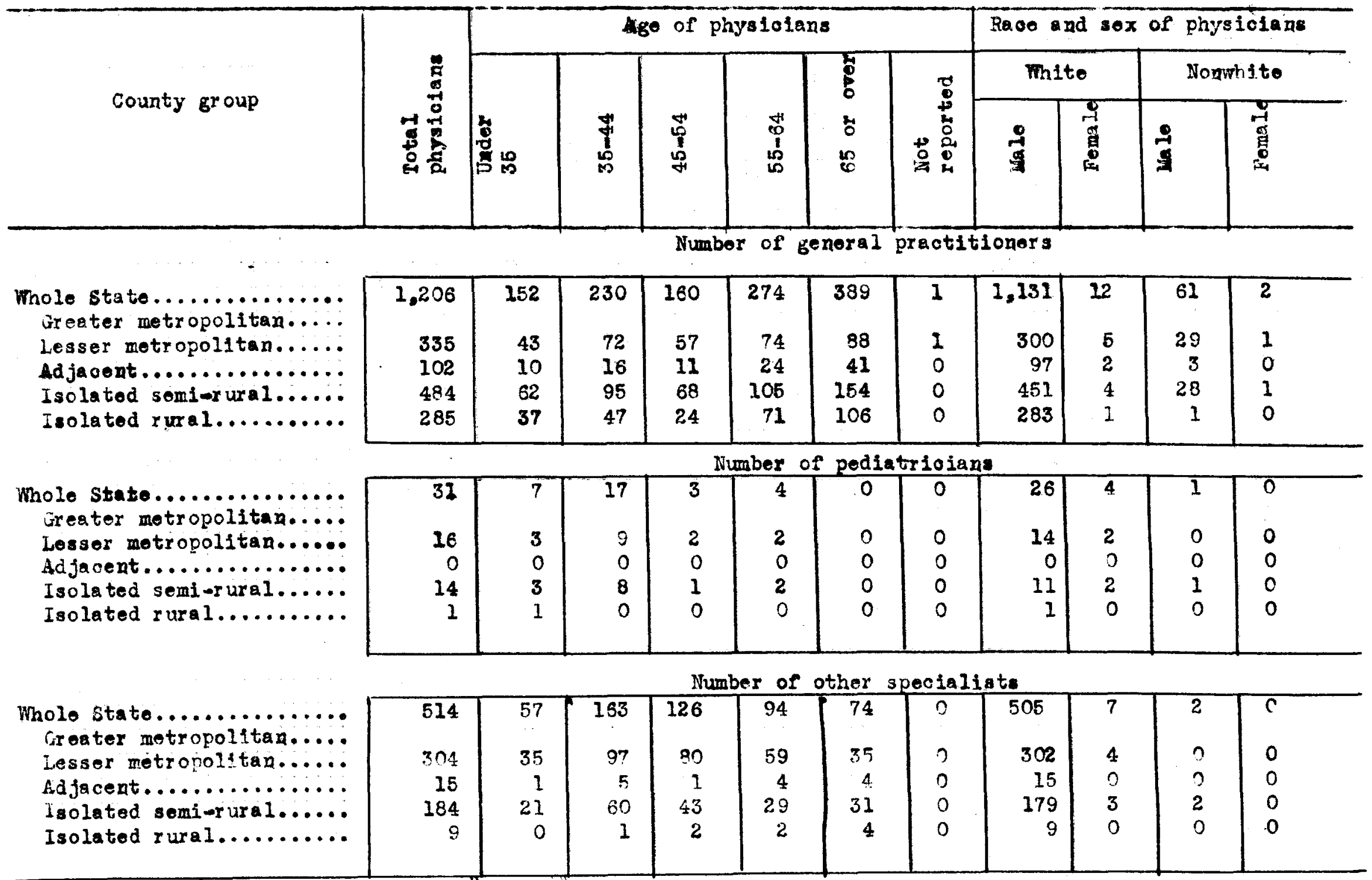


Table 13. Liedical trasing of toneral practitioners, by age and oounty group in hontuoky

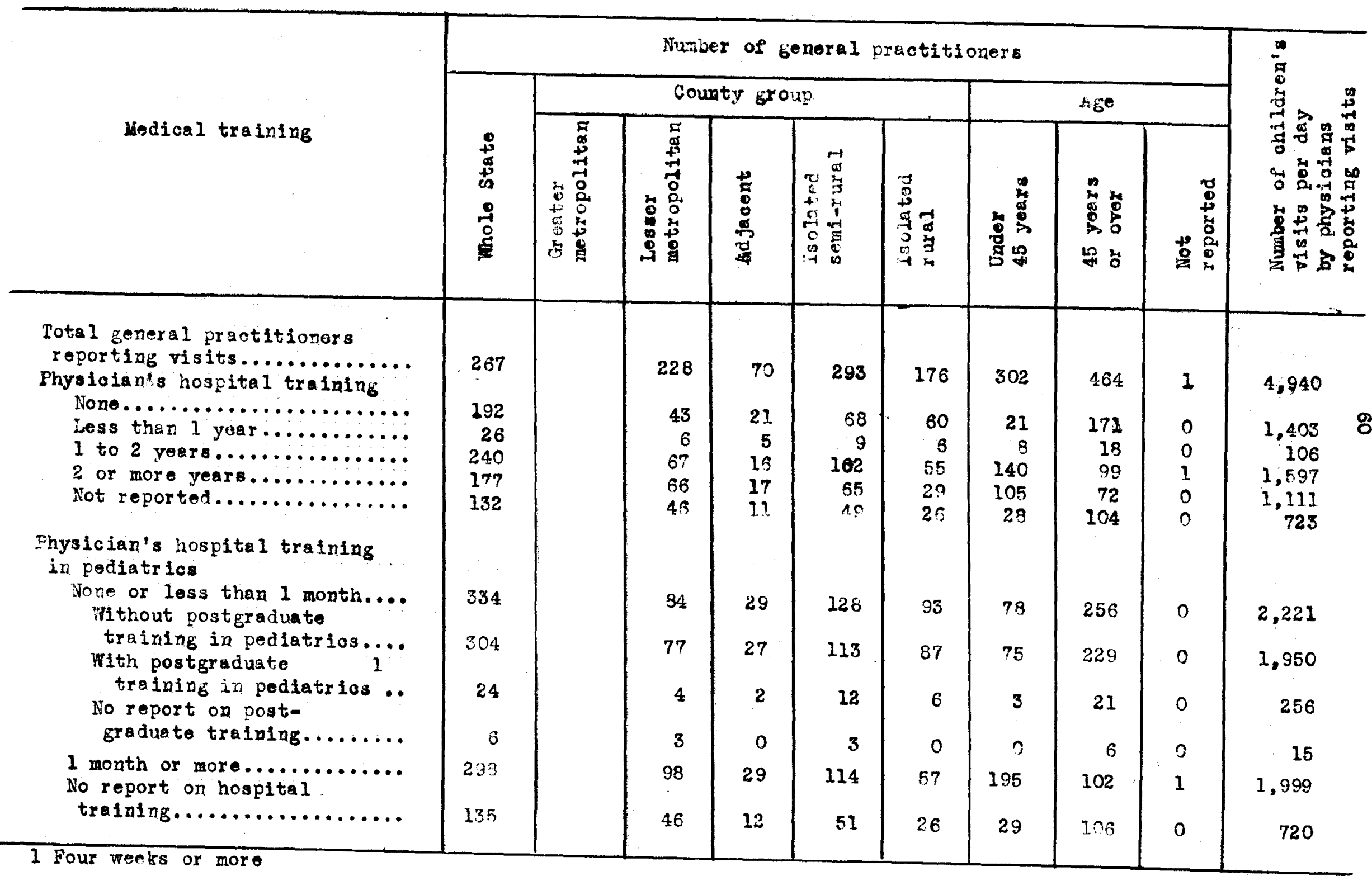


Table 14. Arailability to general practitioners and podiatriolans of hospitals admitting ohildrex. by oounty group in kentuoky.

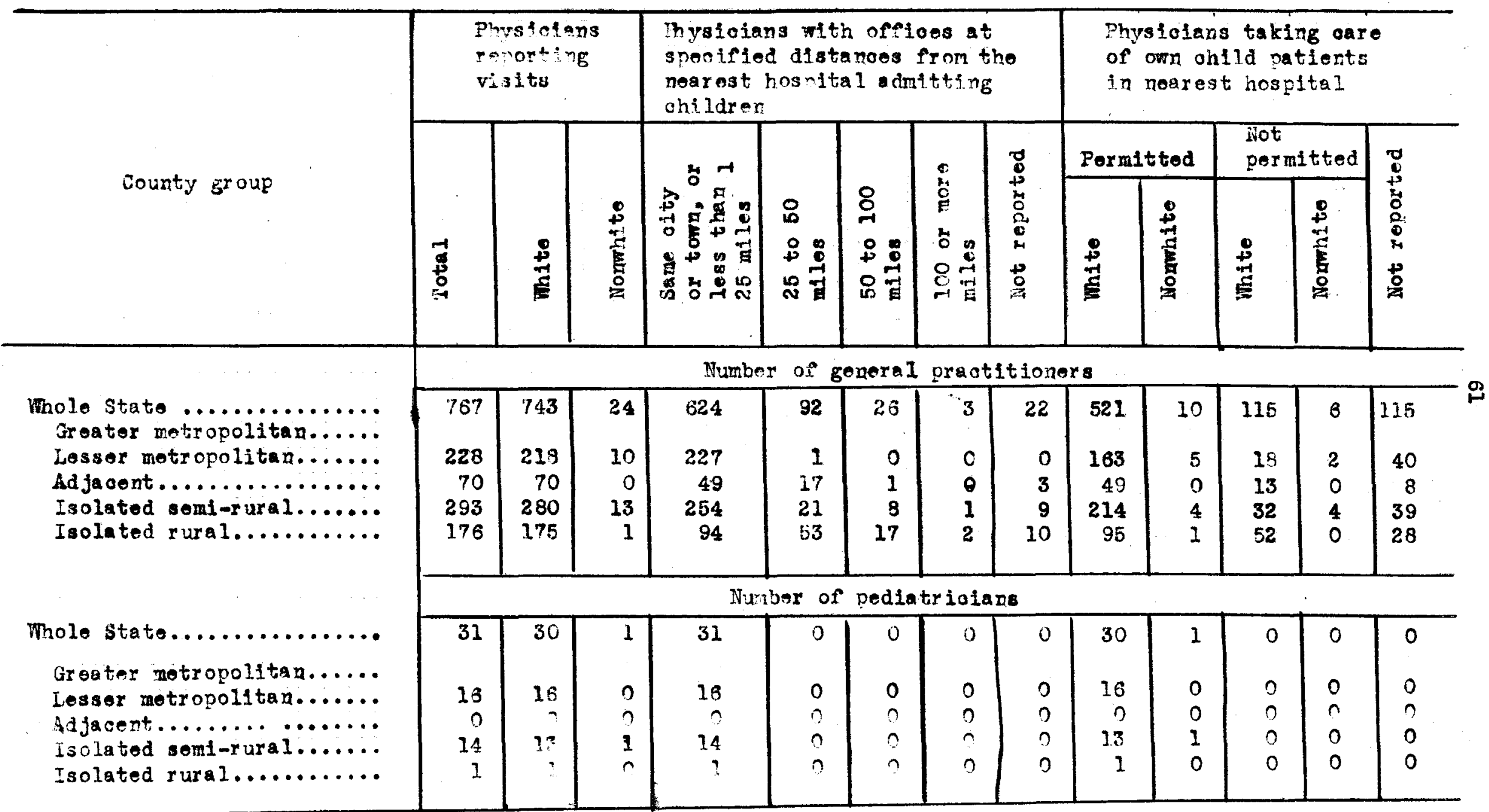

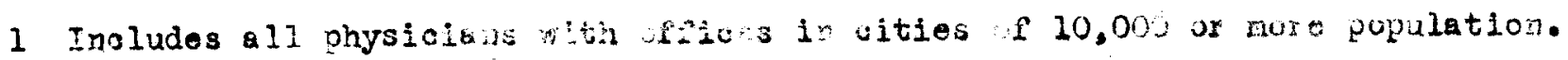


Table 15. Summary of data on physicians in Kentuoky.

\begin{tabular}{|c|c|c|c|}
\hline & $\begin{array}{l}\text { Goneral } \\
\text { practitioners }\end{array}$ & $\begin{array}{l}\text { Podiatr 1- } \\
\text { oians }\end{array}$ & $\begin{array}{l}\text { Other } \\
\text { Bpecialists }\end{array}$ \\
\hline $\begin{array}{l}\text { a. Number of visits to persons of a } 11 \text { agen.... } \\
\text { Number of visits to children............. } \\
\text { Number of physicians reporting } \ldots \ldots \ldots \ldots \ldots\end{array}$ & $\begin{array}{r}13,493 \\
4,342 \\
677\end{array}$ & $\begin{array}{r}667 \\
622 \\
31\end{array}$ & $\begin{array}{r}4,362 \\
552 \\
\\
317 \\
\end{array}$ \\
\hline 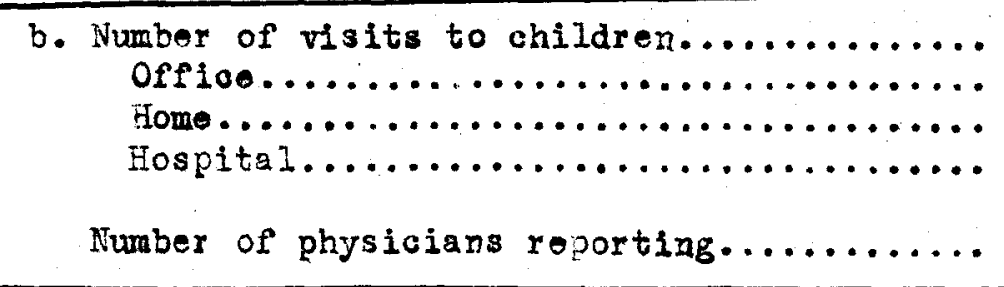 & $\begin{array}{r}4,361 \\
2,889 \\
951 \\
521 \\
689\end{array}$ & $\begin{array}{r}622 \\
377 \\
90 \\
155 \\
31\end{array}$ & $\begin{array}{r}618 \\
372 \\
21 \\
225 \\
348\end{array}$ \\
\hline 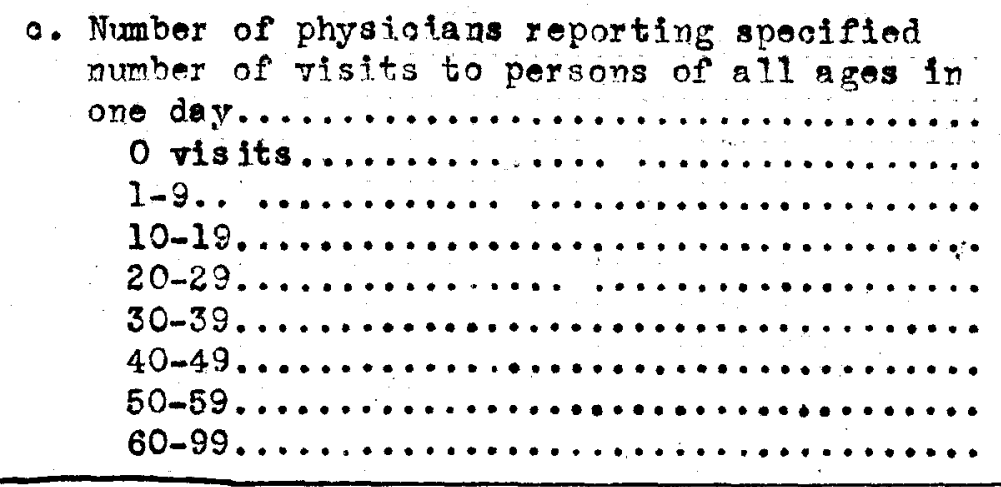 & $\begin{array}{r}677 \\
92 \\
115 \\
166 \\
134 \\
95 \\
35 \\
19 \\
21\end{array}$ & $\begin{array}{r}31 \\
0 \\
6 \\
11 \\
6 \\
5 \\
1 \\
2 \\
0\end{array}$ & $\begin{array}{r}317 \\
93 \\
61 \\
73 \\
43 \\
24 \\
17 \\
3 \\
3\end{array}$ \\
\hline 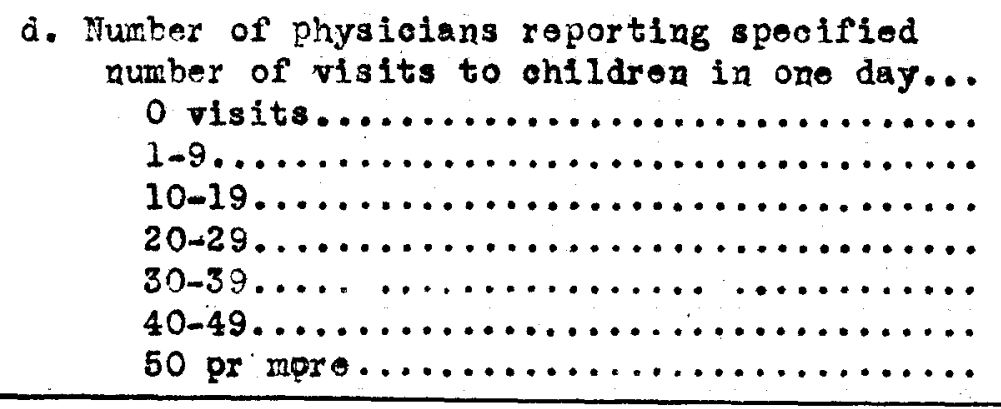 & $\begin{array}{r}767 \\
151 \\
427 \\
156 \\
18 \\
10 \\
5 \\
\times \\
\end{array}$ & $\begin{array}{r}31 \\
0 \\
7 \\
13 \\
3 \\
6 \\
1 \\
1\end{array}$ & $\begin{array}{r}368 \\
212 \\
134 \\
20 \\
2 \\
0 \\
0 \\
x\end{array}$ \\
\hline $\begin{array}{l}\text { - Number of physioians who care for child } \\
\text { patients for: } \\
\text { Nijor sugery } \ldots \ldots \ldots \ldots \ldots \ldots \ldots \ldots \ldots \ldots \ldots \ldots \ldots \\
\text { Tonsillectomies } \ldots \ldots \ldots \ldots \ldots \ldots \ldots \ldots \ldots \ldots \ldots \\
\text { Feeding problems } \ldots \ldots \ldots \ldots \ldots \ldots \ldots \ldots \ldots \ldots \\
\text { Number of physicians roporting } \ldots \ldots \ldots \ldots \ldots \ldots\end{array}$ & $\begin{array}{l}134 \\
237 \\
554 \\
690\end{array}$ & $\begin{array}{l}x \\
x \\
x\end{array}$ & $\begin{array}{l}x \\
x \\
x\end{array}$ \\
\hline 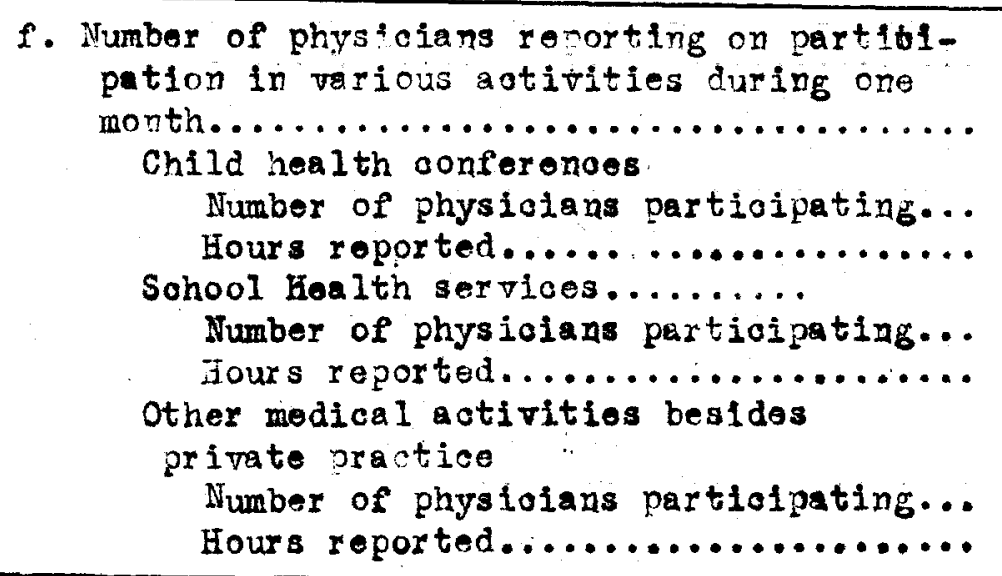 & $\begin{array}{r}537 \\
24 \\
388 \\
34 \\
243 \\
\\
50 \\
895\end{array}$ & $\begin{array}{r}31 \\
3 \\
44 \\
4 \\
35 \\
\\
\\
22 \\
735\end{array}$ & $\begin{array}{l}\mathrm{x} \\
\mathrm{x} \\
\mathrm{x} \\
\mathrm{x} \\
\mathrm{x}\end{array}$ \\
\hline
\end{tabular}


Table 16. Sumary of data on pediatricians in Kentuoky.

Number of pediatrioians roporting hospital

internship and rosidenoy, total.............. 31

None or less than 1 year.................... 2

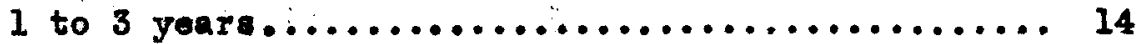

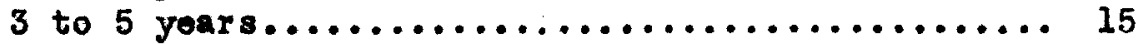

5 or more years.......................... 0

Number of pediatrioians reporting hospltal

training in podiatrios, total................ 30

Nono or loss than 1 year .................... 7

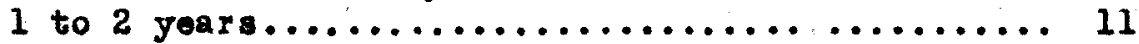

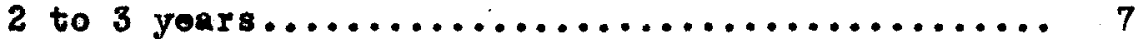

3 or more years......................... 5

Number of pediatricians reporting postgraduate

training in podiatrios, total............... 27

Nono or less than 6 moeks................... 21

6 or moro woeks......................... 6

Number of pediatriolans reporting pediatrio

speolalty, total........................ 29

Allergy................................ 0

Psyohiatry and $/$ or dehavoir problems............ 0

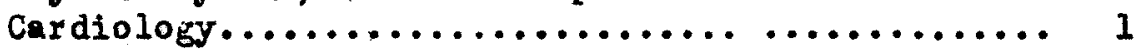

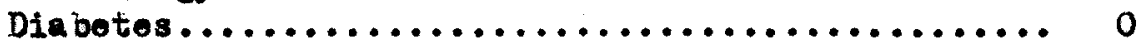

Surgery (inoluding EENT) .................. 0

Dermatology and/or syphilogy................ 0

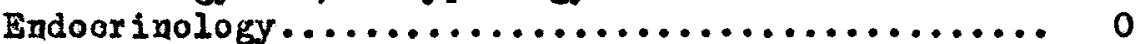

No spocialty........................... 28

Number of podiatrioians roporting spooffied

caro for ohild pationts, total............... 31

Fraotures............................. 3

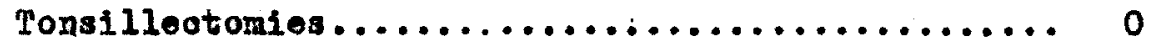

Minor surgery.......................... 14

Number of pediatrioians reporting distance to

aoarest hospital adnitting ohildren with

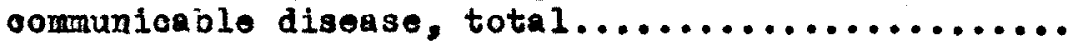

Same city or town, or less than 25 miles........ 28

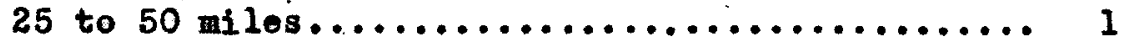

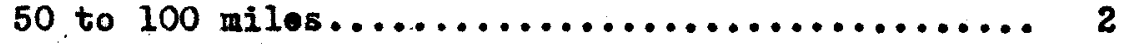

100 miles or more.......................

Number of pediatrioians by avallability of

specified labratory services:

Blood counts $\ldots \ldots \ldots \ldots \ldots \ldots \ldots$

Urinalyses..................

Blood chemistry..............

Hemolytic streptoooccus culture

$X$-ray and/or fluor oscopy.......

Elootrocardiography.
Readily

avallable

31

31

30

29

31

29
Not avilable

0

0

1

2

0

2
Not reported

0

0

0

0

0 
Table 17. He, race and sex of dentists in prirate praotios, by county group in Kontuoky.

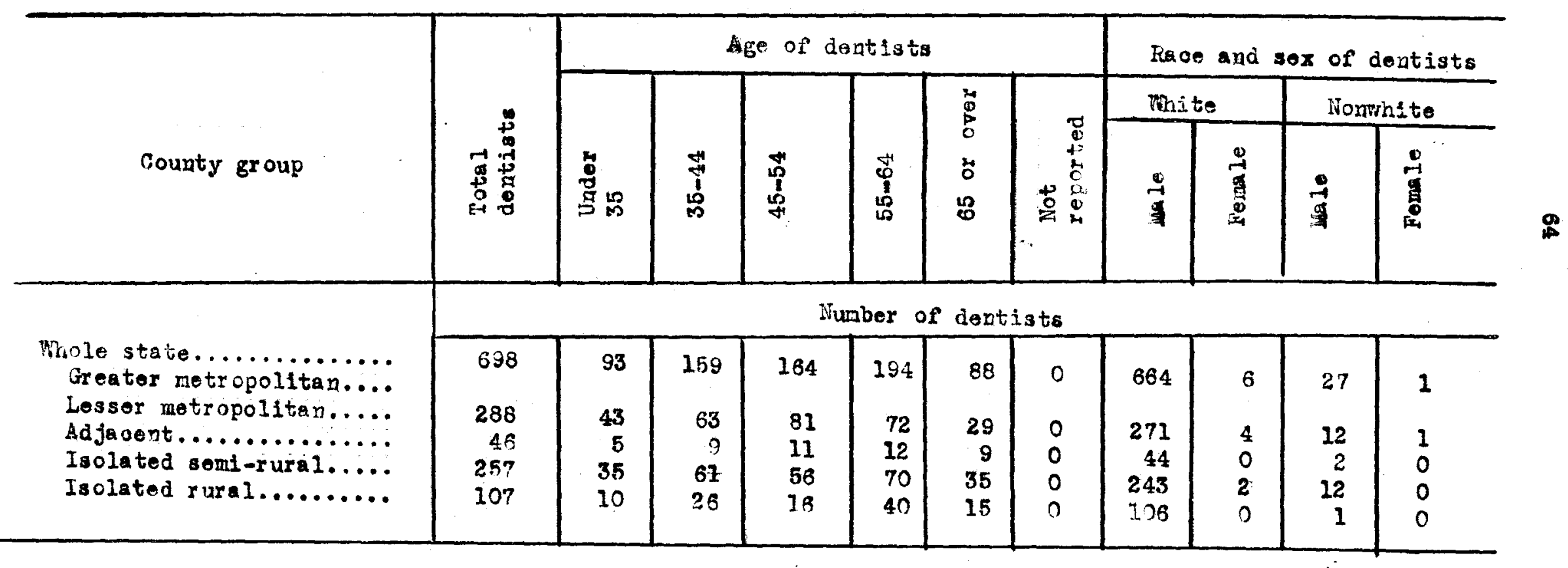


Table 19. Sumary of data on deatists in hontucky.

a. Number of persons of all a ges seon................

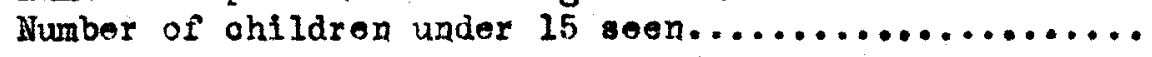

Number of dontists reporting.................... 408

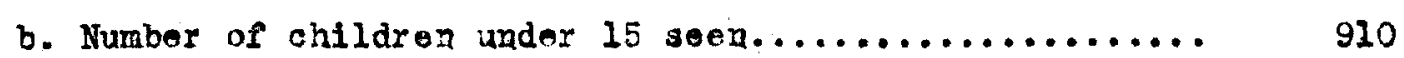
Under 6 years.......................... 218

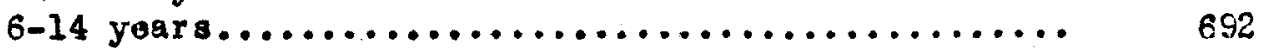

Number of dontists reporting................. 408

c. Number of dentists reporting spooffied number of persons of all ages seen in one day..............

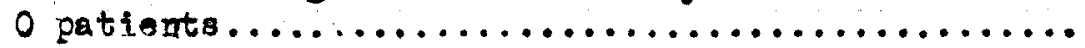

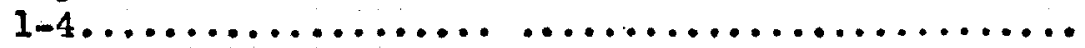

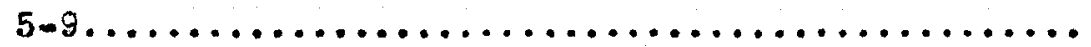

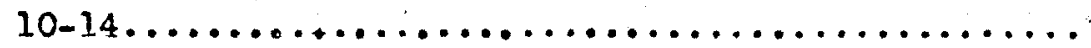

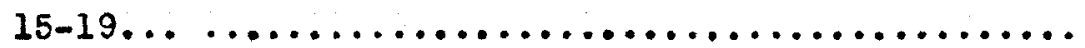

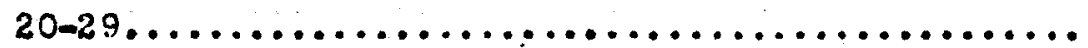
$30-39 \ldots \ldots \ldots \ldots \ldots \ldots \ldots \ldots \ldots \ldots \ldots \ldots \ldots \ldots \ldots \ldots$

408

93

34

111

102

39

22

d. Number of dentists reporting speosfied number of

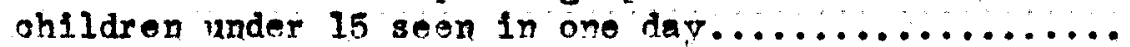

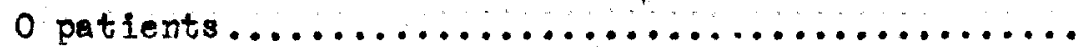

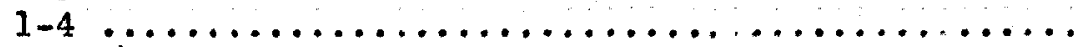

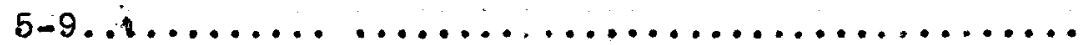

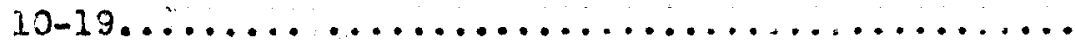
$20-29$

408

172

169

52

14

1

- Humber of dentistmours, all ages................

1,177

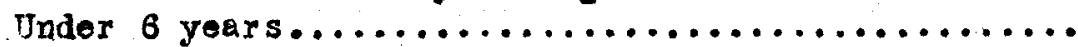

$6-14$ years $\ldots \ldots \ldots \ldots \ldots \ldots \ldots \ldots \ldots \ldots \ldots \ldots \ldots \ldots \ldots$

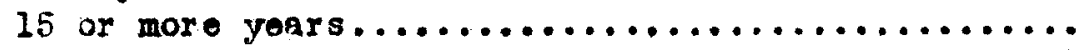

Number of deatists reporting.................

f. Total number of sorvioos, all ages...............

3,606 Under 6 years...........................

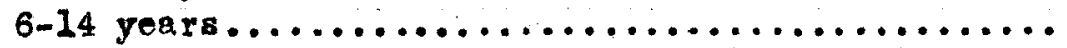
15 or moite years.

2

Number of dentists roporting $\ldots \ldots \ldots \ldots \ldots \ldots \ldots \ldots \ldots$

2,710

287

g. Number of extraotions, a11 ages............... 1,223

Under. 6 years.......................... 63

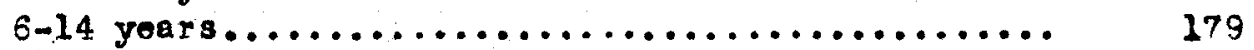

15 or more years........................ 981

1. One-day record of patients seen, dentist-hours and services shown in itoms a through $h$.

2. Same number of dentists reporting in stems $f, g$ and $h$. Total sorvioes inoludes extractions, fillings and other servioes. 
Tablo 19 (oont'd). Sumary of data on dentists in Kontucky.

h. Number of fillings, all ages............... 1,603

Undor 6 yoars............................. 99

$6-14$ yoars............................. 433

15 or nore yoars........................ 1,071

1. Number of dentists reporting whether

children under 15 are seen in private

practioe for service other than

extraotions or emergonoy.....................

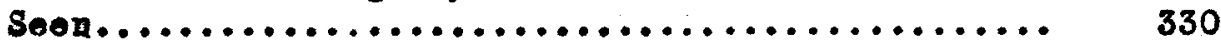

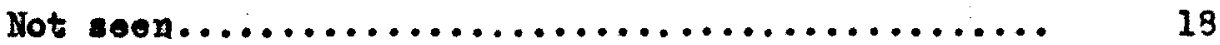

j. Number of dentists roporting specified

number of total offico assistants..............

None.................................

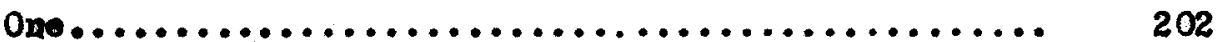

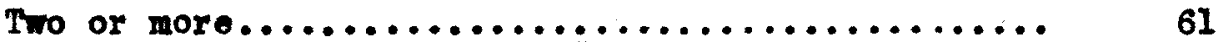

k. Number of dentists reporting specified

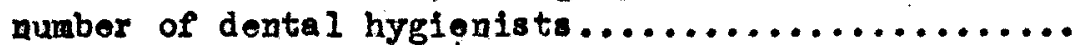

None...................................

One...................................

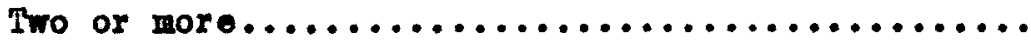

1. Number of dentiste roporting hours during

past four wooks in dental activities

inoluding privato praotico....................

Number of hours apont ins

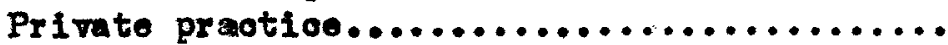

Preschool or sohool dental servloes..........

Other dental activities.

m. Number of dextists reporting hours

during past four wooks in dental

aotivities other than private practice............

Preschool or sohool dental services.

Jumber partioipating.....................

Hours reported........................

Other dentil aotivities.

Number partioipating ...................

Hours roported......................

1. One-day record of patients seen, dentist-hours and servioes shown in items a through $h$. 
Table 20. Number of hospitale in Study, by type, size and oounty group in Kentuoky.

\begin{tabular}{|c|c|c|c|c|c|c|c|c|c|c|}
\hline \multirow{3}{*}{$\begin{array}{l}\text { County group } \\
\text { and size of } \\
\text { hospital }\end{array}$} & \multirow{3}{*}{ 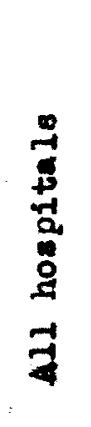 } & \multicolumn{8}{|c|}{ General } & \multirow[b]{3}{*}{$\begin{array}{l}-1 \\
3 \\
0 \\
0 \\
0\end{array}$} \\
\hline & & \multirow[b]{2}{*}{ 垈 } & \multirow{2}{*}{ 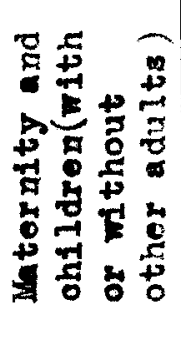 } & \multirow[b]{2}{*}{ 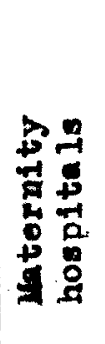 } & \multirow{2}{*}{ 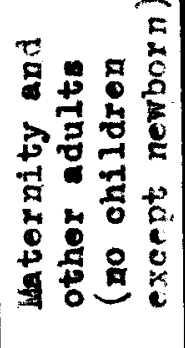 } & \multirow[b]{2}{*}{ 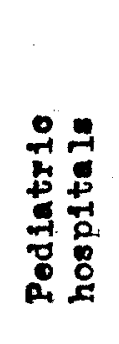 } & \multirow{2}{*}{ 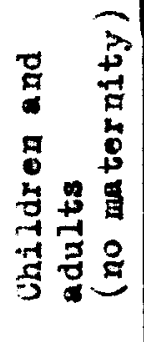 } & \multicolumn{2}{|c|}{$\begin{array}{l}\text { Gospitals } \\
\text { that adnit }\end{array}$} & \\
\hline & & & & & & & & 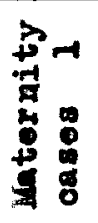 & 赵 & \\
\hline $\begin{array}{l}\text { Whole state.......... } \\
5-24 \text { beds......... } \\
25-99 . \ldots \ldots \ldots \ldots \ldots \\
100-249 . \ldots \ldots \ldots \ldots \\
250 \text { or more........ }\end{array}$ & $\begin{array}{r}103 \\
36 \\
48 \\
13 \\
6\end{array}$ & $\begin{array}{r}97 \\
34 \\
47 \\
11 \\
5\end{array}$ & $\begin{array}{r}38 \\
27 \\
45 \\
11 \\
6\end{array}$ & $\begin{array}{l}3 \\
2 \\
1 \\
0 \\
0\end{array}$ & $\begin{array}{l}1 \\
1 \\
0 \\
0 \\
0\end{array}$ & $\begin{array}{l}1 \\
0 \\
1 \\
0 \\
0\end{array}$ & $\begin{array}{l}4 \\
4 \\
0 \\
0 \\
0\end{array}$ & $\begin{array}{r}92 \\
30 \\
46 \\
11 \\
5\end{array}$ & $\begin{array}{r}93 \\
31 \\
46 \\
11 \\
5\end{array}$ & $\begin{array}{l}6 \\
2 \\
1 \\
2 \\
1\end{array}$ \\
\hline $\begin{array}{l}\text { Hetropolitan and } \\
\text { adjacent counties.. } \\
5-24 \text { beds........ } \\
25-99 \text { beds } \ldots \ldots \ldots \\
100-249 . \ldots \ldots \ldots \\
250 \text { or mor. } \ldots \ldots\end{array}$ & $\begin{array}{r}29 \\
8 \\
9 \\
9 \\
3\end{array}$ & $\begin{array}{r}27 \\
7 \\
9 \\
8 \\
3\end{array}$ & $\begin{array}{r}24 \\
5 \\
8 \\
8 \\
3\end{array}$ & $\begin{array}{l}1 \\
1 \\
0 \\
0 \\
0\end{array}$ & $\begin{array}{l}1 \\
1 \\
0 \\
0 \\
0\end{array}$ & $\begin{array}{l}1 \\
0 \\
1 \\
0 \\
0\end{array}$ & $\begin{array}{l}0 \\
0 \\
0 \\
0 \\
0\end{array}$ & $\begin{array}{r}25 \\
7 \\
8 \\
8 \\
3\end{array}$ & $\begin{array}{r}25 \\
5 \\
9 \\
8 \\
3\end{array}$ & $\begin{array}{l}2 \\
1 \\
0 \\
1 \\
0\end{array}$ \\
\hline $\begin{array}{r}\text { Irolated oounties.... } \\
5-24 \text { beds } \ldots \ldots \ldots \\
25-99 . \ldots \ldots \ldots \\
100-249 . \ldots \ldots \ldots \\
250 \text { or more....... }\end{array}$ & $\begin{array}{r}74 \\
28 \\
39 \\
4 \\
3\end{array}$ & $\begin{array}{r}70 \\
27 \\
38 \\
3 \\
2\end{array}$ & $\begin{array}{r}64 \\
22 \\
3.7 \\
3 \\
2\end{array}$ & $\begin{array}{l}2 \\
1 \\
1 \\
0 \\
0\end{array}$ & $\begin{array}{l}0 \\
0 \\
0 \\
0 \\
0\end{array}$ & $\begin{array}{l}0 \\
0 \\
0 \\
0 \\
0\end{array}$ & $\begin{array}{l}4 \\
4 \\
0 \\
0 \\
0\end{array}$ & $\begin{array}{r}66 \\
23 \\
38 \\
3 \\
2\end{array}$ & $\begin{array}{r}68 \\
26 \\
37 \\
3 \\
2\end{array}$ & $\begin{array}{l}4 \\
1 \\
1 \\
1 \\
1\end{array}$ \\
\hline
\end{tabular}

1. Sum of cols.(3), (4) and (5).

2. Sun of cols. (3), (6) and (7). Inoludes 8 hospitals with oontagious disesso units.

3. See Table 34. Inoludes ome units of goneral hospitals that are reported separately. 
Table 21. Hospital facilities and sorvieos for nowborn, by hospital size and oounty group in Kontuoky.

\begin{tabular}{|c|c|c|c|c|c|c|c|c|}
\hline \multirow[b]{3}{*}{$\begin{array}{l}\text { County group } \\
\text { and size of } \\
\text { hospital }\end{array}$} & \multirow[b]{3}{*}{ 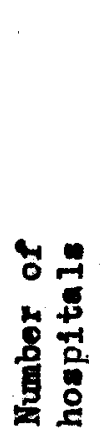 } & \multicolumn{3}{|c|}{ Fuoilities } & \multicolumn{3}{|c|}{ Yowborn during one year } & \multirow{3}{*}{ 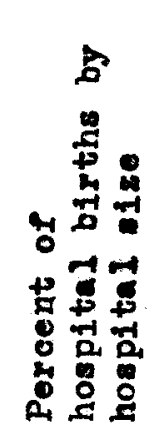 } \\
\hline & & \multirow[b]{2}{*}{$\begin{array}{l}4 \\
0 \\
8 \\
8 \\
8 \\
8\end{array}$} & \multirow[b]{2}{*}{ 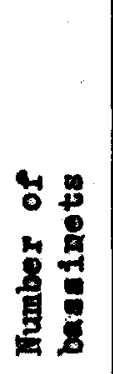 } & \multirow[b]{2}{*}{ 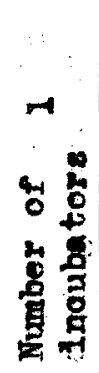 } & \multirow{2}{*}{ 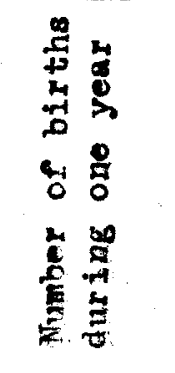 } & \multicolumn{2}{|c|}{ Daye of eare } & \\
\hline & & & & & & है & 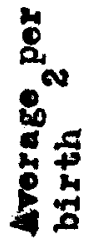 & \\
\hline 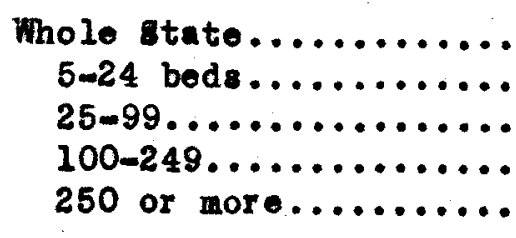 & $\begin{array}{r}97 \\
34 \\
47 \\
11 \\
5\end{array}$ & $\begin{array}{r}6088 \\
497 \\
2360 \\
1442 \\
1789\end{array}$ & $\begin{array}{r}1109 \\
147 \\
461 \\
277 \\
224\end{array}$ & $\begin{array}{r}134 \\
14 \\
51 \\
43 \\
26\end{array}$ & $\begin{array}{r}27,059 \\
2,349 \\
10,164 \\
9,084 \\
5,462\end{array}$ & $\begin{array}{r}206,954 \\
16,232 \\
74,424 \\
65,805 \\
50,493\end{array}$ & $\begin{array}{l}7.6 \\
6.9 \\
7.3 \\
7.2 \\
9.2\end{array}$ & $\begin{array}{c}100.0 \% \\
8.7 \\
37.5 \\
33.6 \\
20.2\end{array}$ \\
\hline 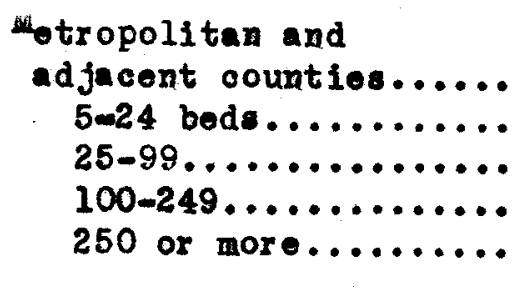 & $\begin{array}{r}27 \\
7 \\
9 \\
8 \\
3\end{array}$ & $\begin{array}{r}2960 \\
100 \\
536 \\
1091 \\
1233\end{array}$ & $\begin{array}{r}552 \\
50 \\
109 \\
230 \\
163\end{array}$ & $\begin{array}{r}73 \\
1 \\
16 \\
37 \\
20\end{array}$ & $\begin{array}{r}14.169 \\
512 \\
2.070 \\
7.721 \\
3.866\end{array}$ & $\begin{array}{r}112,578 \\
4,129 \\
14,924 \\
57,234 \\
36,291\end{array}$ & $\begin{array}{l}7.9 \\
8.1 \\
7.2 \\
7.4 \\
9.4\end{array}$ & $\begin{array}{r}100.0 \\
3.6 \\
14.6 \\
54.5 \\
27.3\end{array}$ \\
\hline 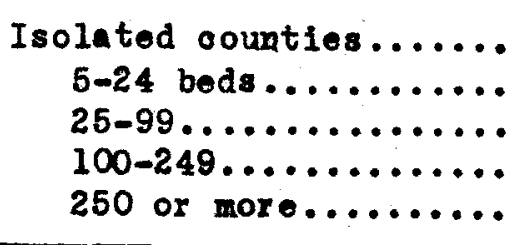 & $\begin{array}{r}70 \\
27 \\
38 \\
3 \\
2\end{array}$ & $\begin{array}{r}3128 \\
397 \\
1324 \\
351 \\
556\end{array}$ & $\begin{array}{r}557 \\
97 \\
352 \\
47 \\
61\end{array}$ & $\begin{array}{r}61 \\
13 \\
36 \\
6 \\
6\end{array}$ & $\begin{array}{r}12,890 \\
1,837 \\
8,094 \\
1,363 \\
1,596\end{array}$ & $\begin{array}{r}94.376 \\
12.103 \\
59,500 \\
8.571 \\
14.202\end{array}$ & $\begin{array}{l}7.3 \\
6.6 \\
7.4 \\
6.3 \\
8.9\end{array}$ & $\begin{array}{r}100.0 \\
14.3 \\
62.7 \\
10.6 \\
12.4\end{array}$ \\
\hline
\end{tabular}

1. Tabulated as zero for 10 hospitals not reporting

2. Col. (6) divided by col. (5). 
Table 22. Faollities and servioes for children in general hospitals, by hospital size and county group in Kontucky.

\begin{tabular}{|c|c|c|c|c|c|c|c|c|c|c|}
\hline \multirow[b]{3}{*}{$\begin{array}{l}\text { County group } \\
\text { and size of } \\
\text { hospital }\end{array}$} & \multicolumn{3}{|c|}{ Hill hospitals } & \multicolumn{4}{|c|}{$\begin{array}{l}\text { Hospitals with } \\
\text { podiatrio ugit: } 2\end{array}$} & \multicolumn{3}{|c|}{$\begin{array}{l}\text { Hoopitale admitting } \\
\text { ohildron only } \\
\text { (pedfatrio hospitals) }\end{array}$} \\
\hline & \multirow[b]{2}{*}{ 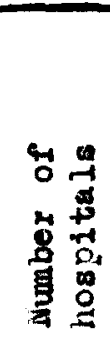 } & \multirow[b]{2}{*}{ 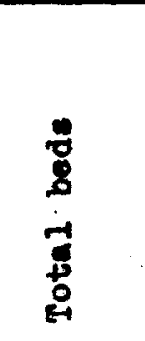 } & \multirow{2}{*}{ 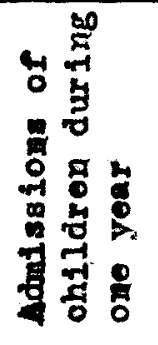 } & \multirow[b]{2}{*}{$\begin{array}{l}4 \\
0\end{array}$} & \multicolumn{2}{|c|}{ Number of bede } & \multirow{2}{*}{ 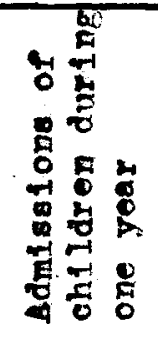 } & \multirow{2}{*}{ 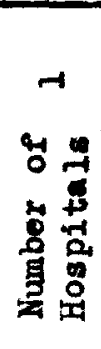 } & \multirow[b]{2}{*}{ 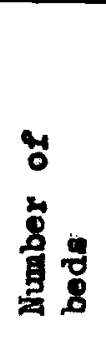 } & \multirow{2}{*}{ 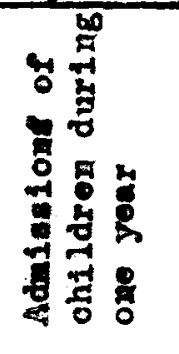 } \\
\hline & & & & & 荵 & 总 & & & & \\
\hline 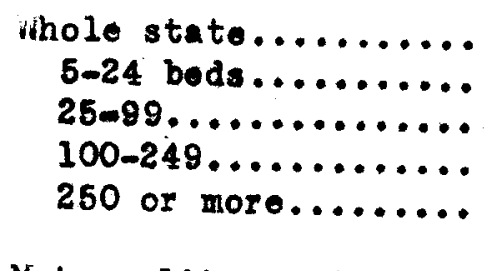 & $\begin{array}{r}97 \\
34 \\
47 \\
11 \\
5\end{array}$ & $\begin{array}{r}6,083 \\
497 \\
2,360 \\
1,442 \\
1,789\end{array}$ & $\begin{array}{r}24,164 \\
1,785 \\
11,409 \\
4,361 \\
6,609\end{array}$ & $\begin{array}{r}11 \\
0 \\
4 \\
2 \\
5\end{array}$ & $\begin{array}{r}2,318 \\
0 \\
305 \\
219 \\
1,789\end{array}$ & $\begin{array}{r}303 \\
0 \\
92 \\
21 \\
190\end{array}$ & $\begin{array}{r}10,819 \\
0 \\
2,974 \\
1,236 \\
6,609\end{array}$ & $\begin{array}{l}1 \\
0 \\
1 \\
0 \\
0\end{array}$ & $\begin{array}{r}75 \\
0 \\
75 \\
0 \\
0\end{array}$ & $\begin{array}{r}1,967 \\
0 \\
1,967 \\
0 \\
0\end{array}$ \\
\hline 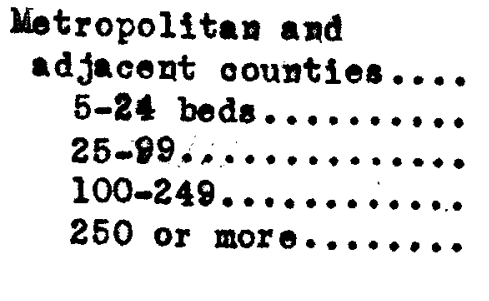 & $\begin{array}{r}27 \\
7 \\
9 \\
8 \\
3\end{array}$ & $\begin{array}{r}2,960 \\
100 \\
536 \\
1,091 \\
1,233\end{array}$ & $\begin{array}{r}11,331 \\
360 \\
3,207 \\
3,140 \\
4,624\end{array}$ & $\begin{array}{l}7 \\
0 \\
3 \\
1 \\
3\end{array}$ & $\begin{array}{r}1,598 \\
0 \\
258 \\
107 \\
1,233\end{array}$ & $\begin{array}{r}246 \\
0 \\
86 \\
15 \\
145\end{array}$ & $\begin{array}{r}7,909 \\
0 \\
2,839 \\
446 \\
4,624\end{array}$ & $\begin{array}{l}1 \\
0 \\
1 \\
0 \\
0\end{array}$ & $\begin{array}{r}75 \\
0 \\
75 \\
0 \\
0\end{array}$ & $\begin{array}{r}1,957 \\
0 \\
1,957 \\
0 \\
0\end{array}$ \\
\hline $\begin{array}{r}\text { Isolated oounties..... } \\
5-24 \text { beds.......... } \\
25-99 . \ldots \ldots \ldots \ldots \ldots \\
100-249 . \ldots \ldots \ldots \ldots \\
250 \text { or more........ }\end{array}$ & $\begin{array}{r}70 \\
27 \\
38 \\
3 \\
2\end{array}$ & $\begin{array}{r}3,128 \\
397 \\
1,824 \\
351 \\
556\end{array}$ & $\begin{array}{r}12,833 \\
1,425 \\
8,202 \\
1,221 \\
1,985\end{array}$ & $\begin{array}{l}4 \\
0 \\
1 \\
1 \\
2\end{array}$ & $\begin{array}{r}715 \\
0 \\
47 \\
112 \\
556\end{array}$ & $\begin{array}{r}57 \\
0 \\
6 \\
6 \\
45\end{array}$ & $\begin{array}{r}2,910 \\
0 \\
135 \\
790 \\
1,985\end{array}$ & $\begin{array}{l}0 \\
0 \\
0 \\
0 \\
0\end{array}$ & $\begin{array}{l}0 \\
0 \\
0 \\
9 \\
0\end{array}$ & $\begin{array}{l}0 . \\
0 \\
0 \\
0 \\
0\end{array}$ \\
\hline
\end{tabular}

1. Same as Table 20, 001. (6).

2. Includes pediatrio hospitals. 
Table 23. Child admission rates in genoral hospitals, by hospital size and county group in Kentuoky.

\begin{tabular}{|c|c|c|c|c|c|c|c|}
\hline \multirow[b]{2}{*}{$\begin{array}{l}\text { County group } \\
\text { and ise of } \\
\text { hospital }\end{array}$} & \multirow[b]{2}{*}{$\begin{array}{l}\text { Number of } \\
\text { hospitals }\end{array}$} & \multirow[b]{2}{*}{$\begin{array}{l}\text { Child } \\
\text { adnissions } \\
\text { por bed } \\
\text { during one } \\
\text { yoar }\end{array}$} & \multicolumn{2}{|c|}{$\begin{array}{l}\text { Poroent of ohild } \\
\text { admisaions that } \\
\text { are in - }\end{array}$} & \multirow[b]{2}{*}{$\begin{array}{l}\text { Peroent } \\
\text { of ohild } \\
\text { admissiors } \\
\text { oy hospital } \\
\text { size }\end{array}$} & \multicolumn{2}{|c|}{$\begin{array}{l}\text { Number por } 100,000 \\
\text { childr on }\end{array}$} \\
\hline & & & $\begin{array}{l}\text { Hospitals } \\
\text { with } \\
\text { pediatrico } \\
\text { imits }\end{array}$ & $\begin{array}{l}\text { Pediatrio } \\
\text { hospitals: }\end{array}$ & & $\begin{array}{r}\text { Total } \\
\text { bede }\end{array}$ & $\begin{array}{l}\text { Total ohild } \\
\text { admissions } \\
\text { during one } \\
\text { year }\end{array}$ \\
\hline 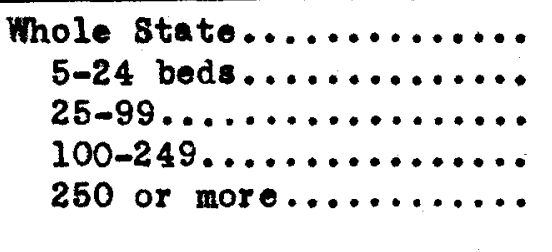 & $\begin{array}{r}97 \\
34 \\
47 \\
11 \\
5\end{array}$ & $\begin{array}{l}4.0 \\
3.6 \\
4.8 \\
3.0 \\
3.7\end{array}$ & $\begin{array}{r}44.8 \\
26.1 \\
28.3 \\
100.0\end{array}$ & $\begin{array}{r}8.1 \\
=-\overline{2} \\
17.2 \\
--\end{array}$ & $\begin{array}{r}100.0 \\
7.4 \\
47.2 \\
18.0 \\
27.4\end{array}$ & $\begin{array}{r}690.3 \\
-- \\
-- \\
-- \\
--\end{array}$ & $\begin{array}{r}2740.0 \\
-- \\
-- \\
-- \\
--\end{array}$ \\
\hline $\begin{array}{l}\text { hetropolitan and } \\
\text { adjacent counties....... } \\
5-24 \text { beds............ } \\
25-99 \ldots \ldots \ldots \ldots \ldots \ldots \ldots \\
100-249 \ldots \ldots \ldots \ldots \ldots \ldots \\
250 \text { or more............. }\end{array}$ & $\begin{array}{r}27 \\
7 \\
9 \\
9 \\
3\end{array}$ & $\begin{array}{l}3.8 \\
3.6 \\
6.0 \\
2.9 \\
3.8\end{array}$ & $\begin{array}{r}69.8 \\
--\overline{-} \\
89.5 \\
14.2 \\
100.0\end{array}$ & $\begin{array}{r}17.3 \\
--\overline{0} \\
61.0 \\
--\end{array}$ & $\begin{array}{r}100.0 \\
3.2 \\
28.3 \\
27.7 \\
40.8\end{array}$ & $\begin{array}{r}1203.2 \\
-- \\
-- \\
-- \\
--\end{array}$ & $\begin{array}{r}4605.8 \\
-- \\
-- \\
-- \\
--\end{array}$ \\
\hline $\begin{array}{l}\text { Isolated counties........ } \\
5-24 \text { beds............ } \\
25-99 \ldots \ldots \ldots \ldots \ldots \ldots \ldots \\
100-249 \ldots \ldots \ldots \ldots \ldots \ldots \\
250 \text { or more............ }\end{array}$ & $\begin{array}{r}70 \\
27 \\
38 \\
3 \\
2\end{array}$ & $\begin{array}{l}4.1 \\
3.6 \\
4.5 \\
3.5 \\
3.6\end{array}$ & $\begin{array}{r}22.7 \\
-. \overline{1} \\
1.6 \\
64.7 \\
100.0\end{array}$ & $\begin{array}{l}- \\
-- \\
- \\
- \\
- \\
-\end{array}$ & $\begin{array}{r}100.0 \\
11.1 \\
63.9 \\
9.5 \\
15.5\end{array}$ & $\begin{array}{r}491.9 \\
-- \\
-- \\
-- \\
--\end{array}$ & $\begin{array}{r}2018.1 \\
-- \\
-- \\
-- \\
--\end{array}$ \\
\hline
\end{tabular}


Tablo 24. Hospitals admitting acute polionyelitis cases, by hospital and oounty group in Kentucky.

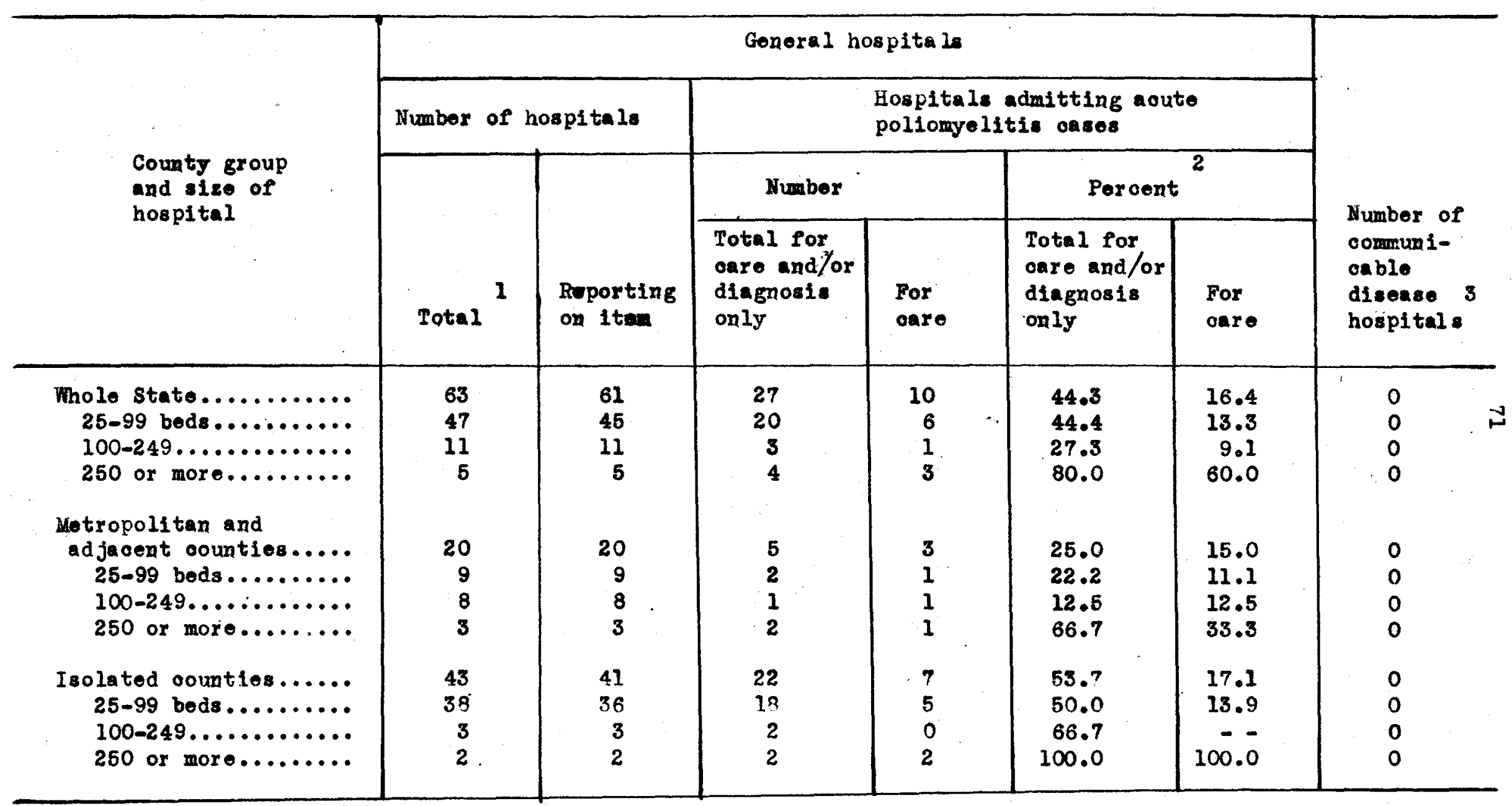

1. Same as Table 20, cod. (2), excluding hospitals of 5-24 beds.

2. Based on hospitals reporting, col. (2).

3. Includes hospitals with 5-24 bods. 
Table 26. Characteristics of small genoral hospitals (5m24 bods), by county group in Kentuoky.

\begin{tabular}{|c|c|c|c|c|c|c|c|c|c|c|c|c|}
\hline \multirow[b]{3}{*}{ Characteristio } & \multicolumn{9}{|c|}{ Number of hospitals } & \multirow{2}{*}{\multicolumn{3}{|c|}{$\begin{array}{l}\text { Perdont of } \\
\text { hospitali with } \\
\text { opecifled ohar- } \\
\text { aoteristio } 1\end{array}$}} \\
\hline & \multicolumn{3}{|c|}{ Whole state } & \multicolumn{3}{|c|}{$\begin{array}{l}\text { lotropolitan and } \\
\text { adjacont counties }\end{array}$} & \multicolumn{3}{|c|}{$\begin{array}{l}\text { Isolated } \\
\text { count los }\end{array}$} & & & \\
\hline & 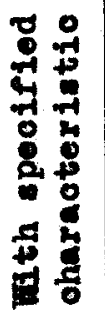 & 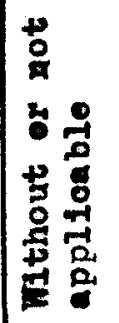 & $\begin{array}{l}0 \\
0 \\
+ \\
0 \\
0 \\
0 \\
0 \\
0 \\
0 \\
0\end{array}$ & 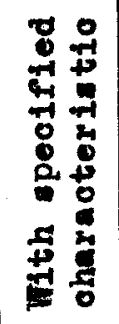 & 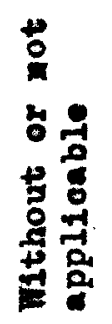 & $\begin{array}{l}8 \\
0 \\
+ \\
0 \\
0 \\
8 \\
0 \\
0 \\
0\end{array}$ & 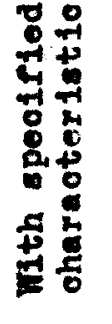 & 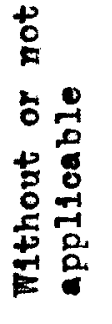 & $\begin{array}{l}8 \\
8 \\
+ \\
0 \\
0 \\
0 \\
0 \\
0 \\
0 \\
0\end{array}$ & $\begin{array}{l}+ \\
\pm \\
\pm \\
9 \\
9 \\
9\end{array}$ & 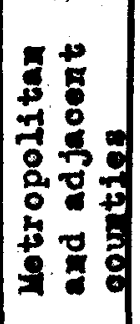 & $\begin{array}{l} \\
0 \\
8 \\
4 \\
4 \\
0 \\
0 \\
0 \\
0\end{array}$ \\
\hline 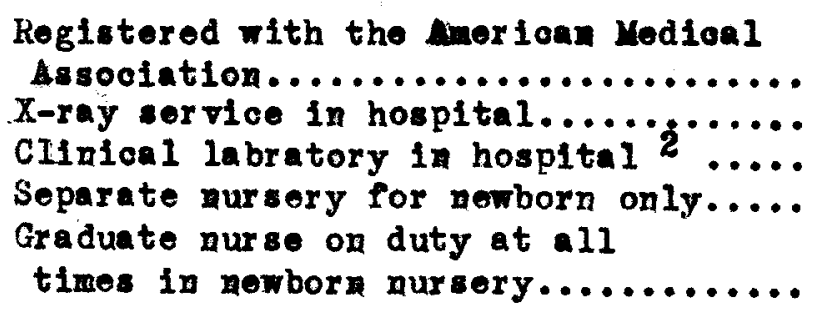 & $\begin{array}{r}15 \\
15 \\
3 \\
22 \\
\\
6\end{array}$ & $\begin{array}{l}19 \\
12 \\
29 \\
12 \\
\\
28\end{array}$ & $\begin{array}{l}0 \\
7 \\
3 \\
0 \\
0\end{array}$ & $\begin{array}{l}3 \\
3 \\
0 \\
4 \\
0\end{array}$ & $\begin{array}{l}4 \\
3 \\
6 \\
3 \\
7\end{array}$ & $\begin{array}{l}0 \\
1 \\
1 \\
0 \\
0\end{array}$ & $\begin{array}{r}12 \\
12 \\
3 \\
18 \\
6\end{array}$ & $\begin{array}{r}15 \\
9 \\
22 \\
9 \\
21\end{array}$ & $\begin{array}{l}0 \\
6 \\
2 \\
0 \\
0\end{array}$ & $\begin{array}{r}44.1 \\
55.6 \\
9.7 \\
64.7 \\
17.6\end{array}$ & $\mid \begin{array}{c}42.9 \\
50.1 \\
-- \\
57.1 \\
--\end{array}$ & $\begin{array}{l}44.4 \\
57.1 \\
12.0 \\
66.7 \\
22.2\end{array}$ \\
\hline
\end{tabular}

1. Not reported oxcluded from computations.

2. At least the following 3 types of sorvioes available - bacteriology, bioohemistry and homatology. 
Table 27. Charaoteristios of care for nowborn infante in largor goneral hospitale (25 or more beds) in Kentuoky.

\begin{tabular}{|c|c|c|c|c|c|c|c|c|}
\hline \multirow[b]{2}{*}{ Characterietie } & \multicolumn{3}{|c|}{ Number of hospitale } & \multicolumn{3}{|c|}{$\begin{array}{l}\text { Number of birthe } \\
\text { during one year }\end{array}$} & \multirow{2}{*}{ 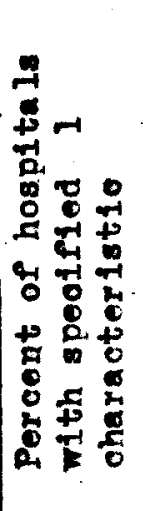 } & \multirow{2}{*}{ 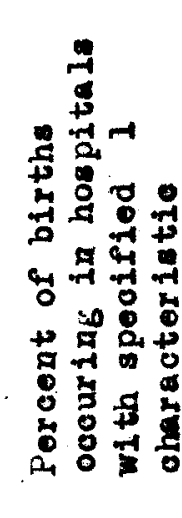 } \\
\hline & 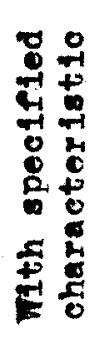 & 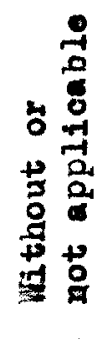 & $\begin{array}{l}0 \\
8 \\
+ \\
0 \\
0 \\
8 \\
0 \\
0 \\
+0 \\
0 \\
0\end{array}$ & 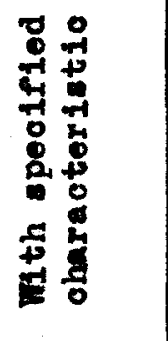 & 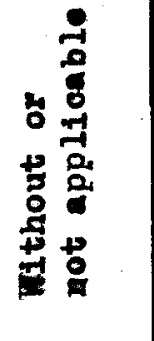 & 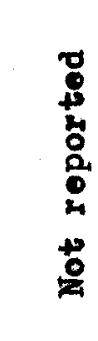 & & \\
\hline 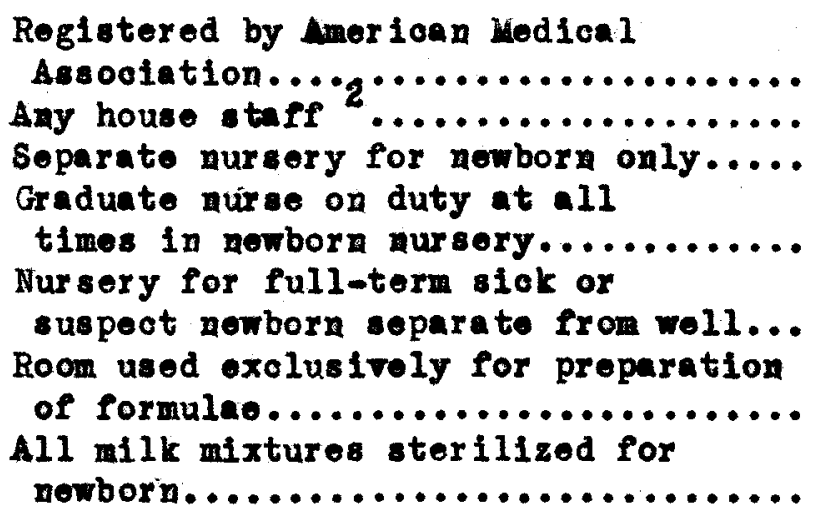 & $\begin{array}{r}56 \\
14 \\
61 \\
\\
43 \\
9 \\
9 \\
24 \\
60\end{array}$ & $\begin{array}{r}7 \\
48 \\
2 \\
18 \\
53 \\
37 \\
1\end{array}$ & $\begin{array}{l}0 \\
1 \\
0 \\
2 \\
1 \\
2 \\
2\end{array}$ & $\begin{array}{r}23,257 \\
12,479 \\
24,358 \\
19,655 \\
6,462 \\
14,190 \\
24,123\end{array}$ & $\begin{array}{r}1,453 \\
11,788 \\
362 \\
4,491 \\
17,816 \\
9,933 \\
0\end{array}$ & $\begin{array}{r}0 \\
443 \\
0 \\
\\
564 \\
443 \\
\\
687 \\
587\end{array}$ & $\begin{array}{l}88.9 \\
22.6 \\
95.2 \\
70.5 \\
14.5 \\
39.3 \\
98.4\end{array}$ & $\begin{array}{r}94.1 \\
51.4 \\
98.6 \\
81.4 \\
26.6 \\
58.8 \\
100.0\end{array}$ \\
\hline
\end{tabular}

1. Not reported exoluded from computations.

2. Inoludes interns, assistant rosidents, residents and fellows. 
Table 28. Characteristioe of oare for sick ohildren in larger genoral hos pitale (25 or more beds) in Kontuoky.

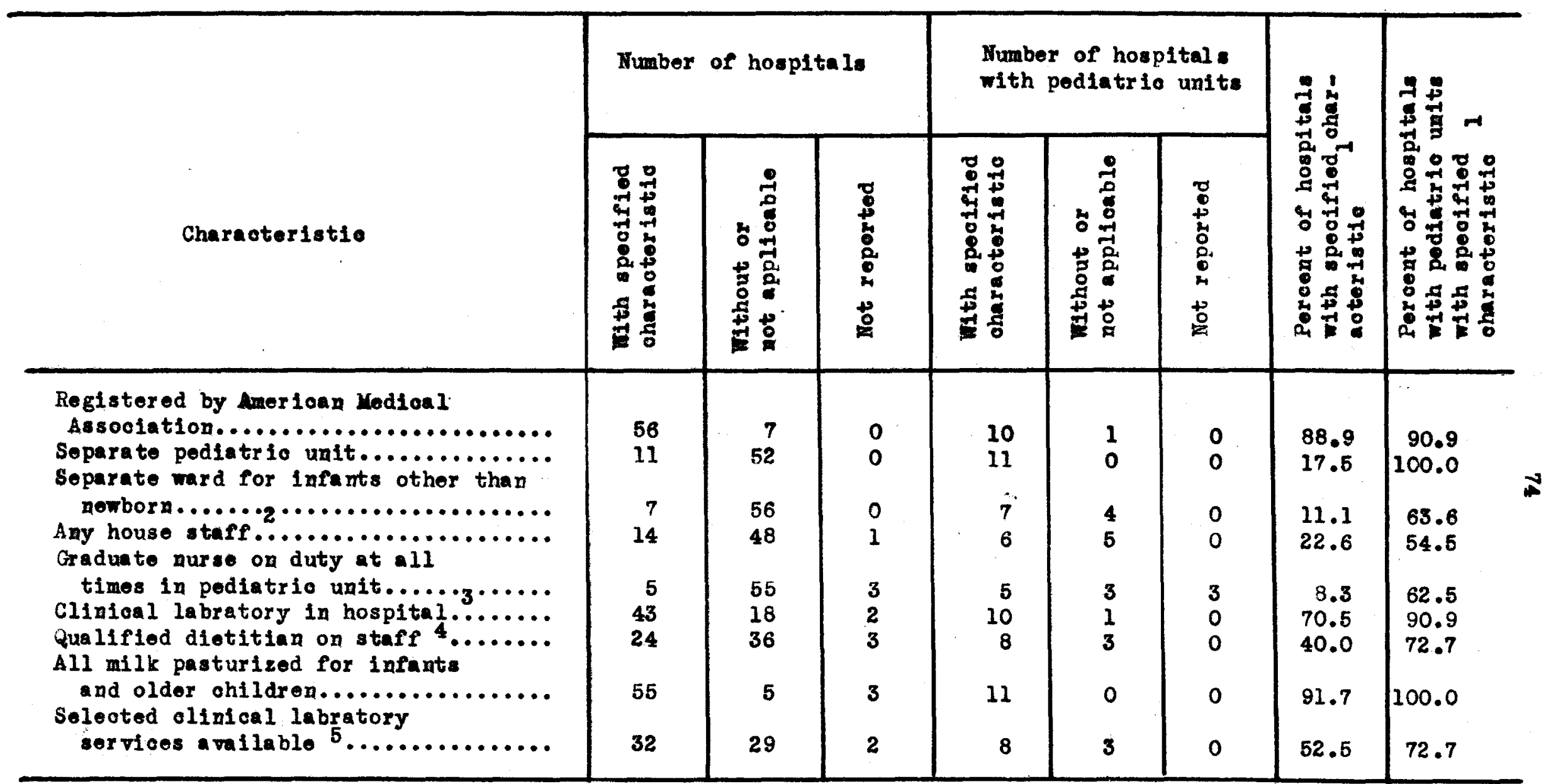

1. Not reported exoluded from computations.

2. Inoludes interns, assistant residents, residents and followe.

3. At least the following threo types of servioes avilable - baoteriology, biochemistry and hematology.

4. Gradutte home oconomist or person with hospital internship in dietetios in oharge of distary department.

5. Blood level for sulfonamides, sedinentation rate, blood oulture and ser un protein. 
Table 29. Child admissions in larger genoral hospitals (25 or more bede) with epecified oharacteristios by oounty group in Kentuoky.

\begin{tabular}{|c|c|c|c|c|c|c|c|c|c|c|c|c|}
\hline \multirow[b]{2}{*}{ Charaoteristie ${ }^{2}$} & \multicolumn{3}{|c|}{ Whole state } & \multicolumn{2}{|c|}{$\begin{array}{l}\text { Wotropolitan and } \\
\text { adjacent oountiea }\end{array}$} & $\begin{array}{c}\text { and } \\
\text { atien }\end{array}$ & \multicolumn{2}{|c|}{ Isolated oountied } & intied & \multicolumn{3}{|c|}{$\begin{array}{l}\text { Poroent of adnis- } \\
\text { sions ooouring } \\
\text { in hospitals } \\
\text { with spoified } \\
\text { charaoteristio }\end{array}$} \\
\hline & 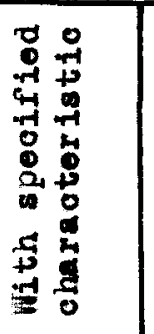 & 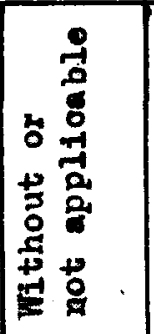 & $\begin{array}{l}0 \\
8 \\
0 \\
0 \\
0 \\
0 \\
2 \\
+0 \\
0\end{array}$ & 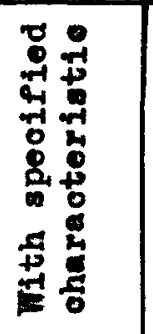 & 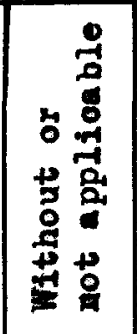 & $\begin{array}{l}8 \\
8 \\
+ \\
0 \\
0 \\
0 \\
0 \\
\pm \\
0 \\
0\end{array}$ & 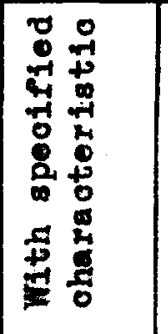 & 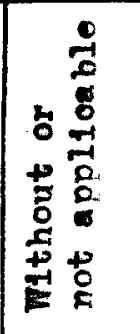 & 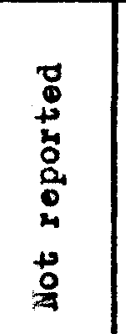 & 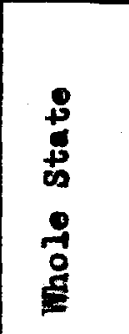 & 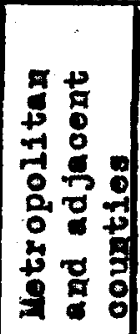 & 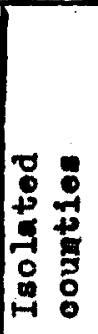 \\
\hline 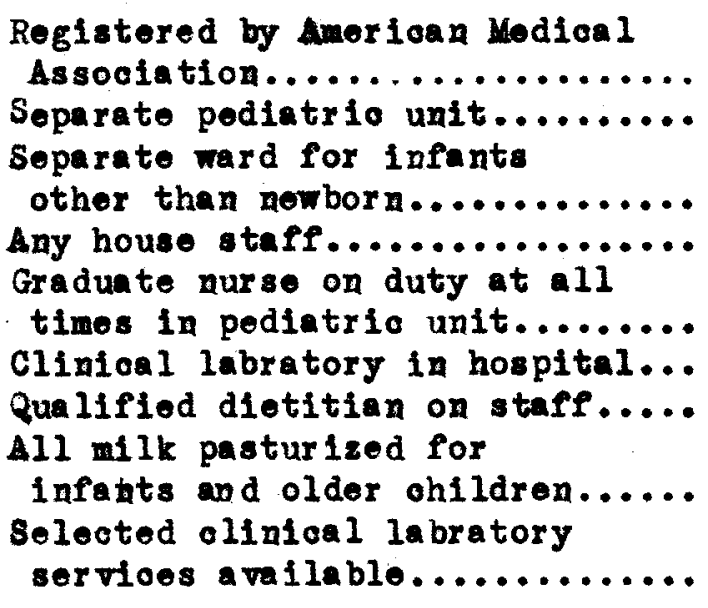 & $\begin{array}{r}21,193 \\
10,819 \\
7,535 \\
10,789 \\
5,565 \\
16,940 \\
12,341 \\
21,177 \\
14,447\end{array}$ & $\begin{array}{r}1,186 \\
11,560 \\
14,844 \\
11,318 \\
15,753 \\
4,942 \\
9,705 \\
860 \\
7,435\end{array}$ & $\begin{array}{r}0 \\
0 \\
0 \\
272 \\
1,061 \\
497 \\
333 \\
342 \\
497\end{array}$ & $\begin{array}{r}10,491 \\
7,909 \\
5,550 \\
8,590 \\
4,775 \\
8,901 \\
8,532 \\
10,971 \\
8,341\end{array}$ & $\begin{array}{r}480 \\
3,062 \\
5,421 \\
2,381 \\
5,270 \\
2,070 \\
2,439 \\
0 \\
2,630\end{array}$ & $\begin{array}{r}0 \\
0 \\
0 \\
0 \\
926 \\
0 \\
0 \\
0 \\
0\end{array}$ & $\begin{array}{r}10,702 \\
2,9108 \\
1,986 \\
2,1998 \\
790 \\
8,039 \\
3,809 \\
10,206 \\
6,106\end{array}$ & $\begin{array}{r}706 \\
8,498 \\
9,423 \\
8,937 \\
10,483 \\
2,872 \\
7,266 \\
860 \\
4,805\end{array}$ & $\begin{array}{c}0 \\
0 \\
\\
0 \\
272 \\
\\
135 \\
497 \\
333 \\
342 \\
497\end{array}$ & $\begin{array}{l}94.7 \\
48.3 \\
33.7 \\
48.8 \\
26.1 \\
77.4 \\
56.0 \\
96.1 \\
66.0\end{array}$ & $\begin{array}{c}95.6 \\
72.1 \\
50.6 \\
78.3 \\
47.5 \\
81.1 \\
77.8 \\
100.0 \\
76.0\end{array}$ & $\begin{array}{r}93.8 \\
25.6 \\
\\
17.4 \\
19.7 \\
\\
7.0 \\
73.7 \\
34.4 \\
92.2 \\
56.0\end{array}$ \\
\hline
\end{tabular}

1. Not reported excluded from computatione.

2. See Table 28 , footmotes $2-5$, for definition of terms. 
Table 31. Hame and lootion of goneral hospitals in Study in Kontuoky.

\begin{tabular}{|c|c|c|}
\hline County & city & Hospital \\
\hline Barren & Glasgow & T. J. Samson Memorial Hospital \\
\hline Bell & Middlesboro & Bvans Fospital \\
\hline Be 11 & Middlesboro & Middlesboro Hosplal \\
\hline Bel1 & Pineville & Pinoville Comunity Hospital \\
\hline$B e 11$ & Beverly & Red Bird Evangelioal Hospital and Health Conter \\
\hline Bour bon & Paris & T. W. Massio Momorial Hospital \\
\hline Boyd & Arhland & Kings Daughters Hospital \\
\hline Boyle & Danville & Ephrain MoDowell Momorial Hospital \\
\hline Caldwell & Prinooton & Pridioeton Hospital \\
\hline Calloway & Murray & Houston-MoDeritt Clinio Hospital \\
\hline Calloway & Murray & Miliam Mason Momorial Hospital \\
\hline Campbell & Dayton & Speers Monor Ial Hos pital \\
\hline Carter & Grayson & J. Q. Stovall Nomorial Hospital \\
\hline Christian & Hopkine ville & Jomale Stuart Memorial Hospital \\
\hline Christian & Hopkinsti11e & Brooks domorial Hospital \\
\hline Christian & Hopkins v1110 & The Moore Clinie \\
\hline Clark & Winchester & Clark County Hospital \\
\hline Clark & Winchester & Guerrant wission Clinic and Hospital \\
\hline clay & Oneida & Oneidn Maternity Hospital \\
\hline Clinton & Albany & Maplo Hill Hospital \\
\hline Crittenden & Mar10x & Crittenden County Hoapital \\
\hline Davies: & Owensboro & Orezsboro-Daviese County Hospital \\
\hline Fayot to & Lexington & Good Samaritan Hospital \\
\hline Fayette & Lexington & St. Joseph's Hospltal \\
\hline Floyd & Martin & Gearhart Hospital \\
\hline Floyd & Prestons bur 8 & Prestonoburg General Hospital \\
\hline Franklin & Frankfort & Kings Daughters Hospltal \\
\hline
\end{tabular}


77

Tablo 31 (cont'd). Name and lootion of general hospitals is Study in Kentuoky.

\begin{tabular}{|c|c|c|}
\hline County & city & Hospital \\
\hline Fulton & Fulton & Fulton Hospital \\
\hline Fulton & Fulton & Jones Clinic and Hospital \\
\hline Graves & Unyfiold & Fuller-Gilliam Hospltal \\
\hline Graves & Mayfield & Mayfield Hospital \\
\hline Har lan & Harlan & Harlan Hospital \\
\hline Har lan & Lymoh & Lymoh Hospital \\
\hline Har lan & Benham & Banham Hospital \\
\hline Har lan & Kenvir & Bleok Mountain Hospital \\
\hline Harlan & Pino Mountain & Pine Mountain Settlemont Hospital \\
\hline Harrison & Cymthiana & Har r ison Momorial Hospital \\
\hline Henders on & Henderson & Henderson Hospital \\
\hline Hopkins & Hadisonvil1e & Hopkins County Hospital \\
\hline Jackson & Annoille & Bussing Momorial Hospital \\
\hline Jeffers on & Louisville & Children's Freo Hospital \\
\hline Jefferson & Loyeis villo & Jowish Hospital \\
\hline Jefforson & Louistille & Kentuoky Baptist Hospital \\
\hline Jefferson & Louis ville & Loulsville General Hoepital \\
\hline Jefferson & Loulstile & Wothodist Doaconess Hospital \\
\hline Jeffers on & Louis ville & Norton Nenorial Infirmary \\
\hline Jofferson & Louisville & Red Cross Hospital \\
\hline Jefferson & Louisville & St. Mary \& Elizaboth Hospital \\
\hline Jefforson & Louistillo & St. Anthony's Hospital \\
\hline Jeffers on & Louisvillo & St. Joseph's Infirmary \\
\hline Jofferson & Loulaville & Susan Speod Davis Homo \& Hospital \\
\hline Johns on & Paintsillo & Paints rillo Hospital \\
\hline Johns on & Paintsoille & Golden Rule Hospltal \\
\hline Johrson & Paintsville & Paintsilile clinio \\
\hline
\end{tabular}


Table 31 (cont!d). Hame and location of general hospitals in Study in Kentuoky.

\begin{tabular}{|c|c|c|}
\hline County & city & Hospital \\
\hline Kenton & Corington & St. Ellzaboth's Hospltal \\
\hline Kenton & Covington : & nim. Booth Momor ial Hospital \\
\hline Knott & Inckoy & Stumbo Momorial Hospital \\
\hline Knox & Barbour vil1e & Kmox Hospital. \\
\hline Laurel & London & Marymount Hospital \\
\hline Lawrence & Louisa & Louisa General Hospital \\
\hline Lawrence & Louise & Riverviow Hospital \\
\hline Leslie & Hyden & $\begin{array}{l}\text { Hyden Hospital and Health Center of } \\
\text { the Montier Nursing Servioe. Ino. }\end{array}$ \\
\hline Letoher & Fleming & Flening Hospital \\
\hline Letcher & Jenkin: & Jonkins Hospital \\
\hline Letoher & Seco & Seoo Hospital \\
\hline Lincoln & Stanford & Stanford Hospital \\
\hline Logan & Russelltille & SImono Nursing Home \\
\hline Mocracken & Paduoah & Riverside Hospital \\
\hline Madison & Berea & Berea College Hospital \\
\hline Madison & Riohnond & GLbson Hospital \\
\hline dison & Riohnond & Patty A. Clay Infirmary \\
\hline Meadison & Riohmond & Henry Cook Pope Hospital \\
\hline tion & Lebanon & Mary Immaculate Infirmary \\
\hline Mas on & Mays ville & Hayswood Hospital \\
\hline Henifoe & Frenchburg & Jane Cook Hospital \\
\hline Mer oer & Harr odsbur $\mathbf{g}$ & A. D. Prloe Memorial Hospital \\
\hline Mont gomery & Wt. Sterling & Mary Chiles Hospital \\
\hline Muhlenber g & Greonvillo & Wuhlenberg Community Hospital \\
\hline Nioholas & Carlisle & Hohnson Momorial Hospital \\
\hline Ohio & Hartford & Crowder Clinio \\
\hline Oldham & La Grange & Mallory-Taylor Wonorial Hospital \\
\hline
\end{tabular}


79

Table 31 (cont'd). Fame and location of general hospitals in Study in Kentuoky.

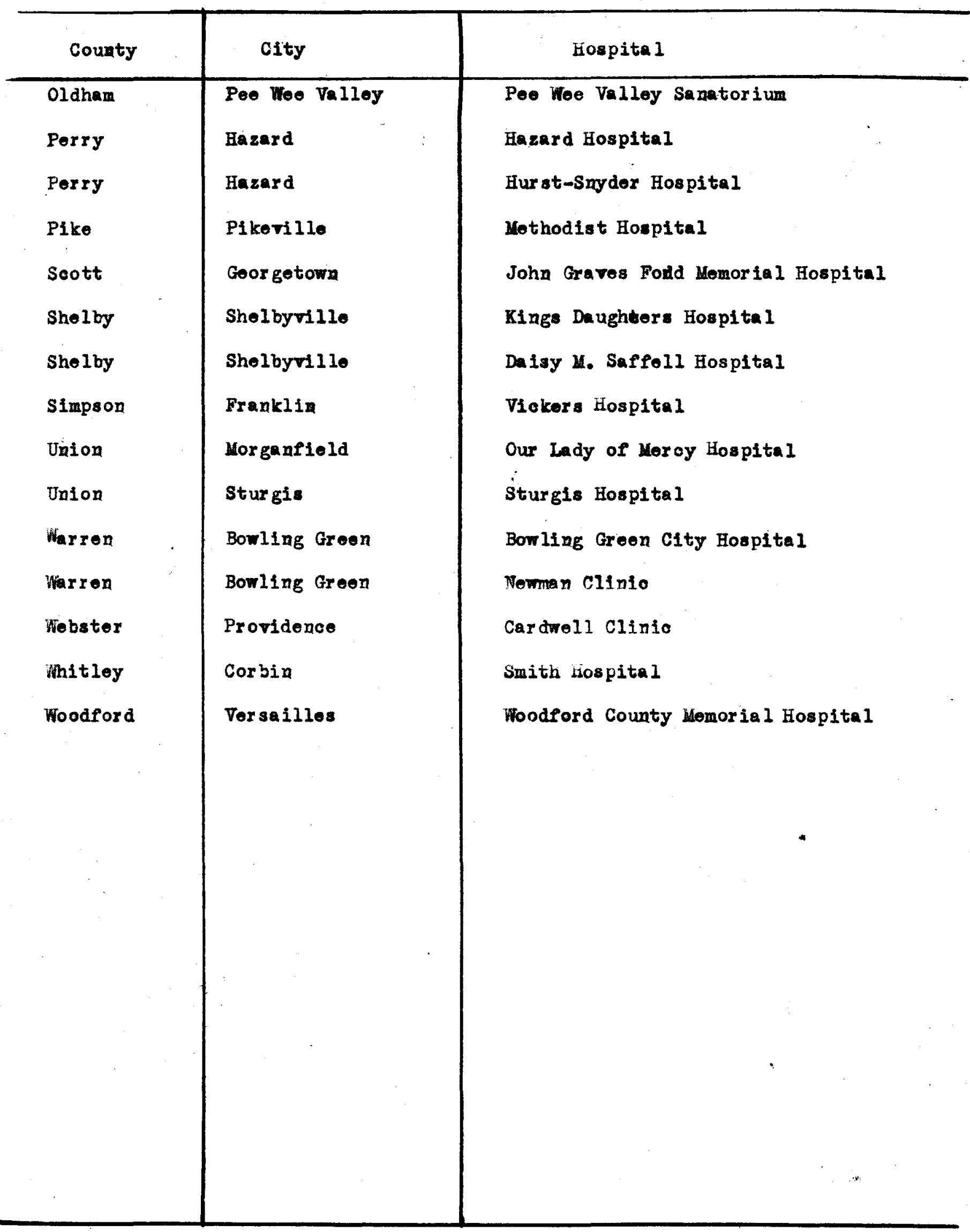


Tablo 32. Sumary of seleoted data related to ohild oare in 97 individual general hospitals in Kentuoky.

Type of service:

Orner ship:

Race of patiente:

1

Acute poliomyelitis cases
Code 1. Mternity and ohildren (with or wh thout other adults) ..............

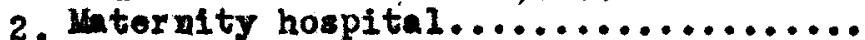
3. Waternity and other adults (no ohildren except nowborn)........

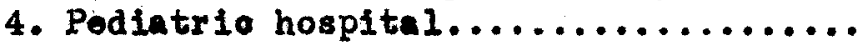

5. Childron ad adult (no tornity)....

Codo 1. Govormental....................

6. Non-governmental : Non-profit.........

8. Non-govermenta l: Proprietary........

Code 1. White only....................

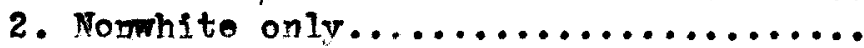

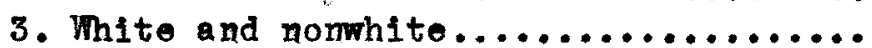

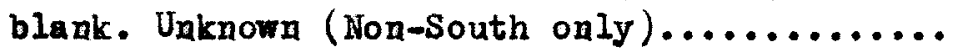

Code 1. Admitted for hospital care...........

2. Adnitted for diagnosis and ersergenoy treatment only............

0. Hot admittod....................

9. No children admitted...............

blank. Unknown.........................
Number of beds, all putients ................................ 6,088

Number of beds, ohildron.....................................

Number of ehildren admitted dur ing one yoar,$\ldots \ldots \ldots \ldots \ldots \ldots \ldots \ldots \ldots \ldots \ldots$

24,164

\begin{tabular}{|c|c|c|c|c|}
\hline Code: & $\frac{Y 08}{(I)}$ & $\frac{\text { No }}{(0)}$ & $\begin{array}{c}\text { Not } \\
\text { Sppleable }\end{array}$ & $\frac{\begin{array}{c}\text { Not } \\
\text { reported }\end{array}}{\text { (blank })}$ \\
\hline & 11 & 82 & 4 & $\mathbf{x}$ \\
\hline & $\begin{array}{l}7 \\
2\end{array}$ & $\begin{array}{r}4 \\
60\end{array}$ & $\begin{array}{c}86 \\
1\end{array}$ & $\begin{array}{l}0 \\
0\end{array}$ \\
\hline & $\begin{array}{r}14 \\
2\end{array}$ & $\begin{array}{l}82 \\
11\end{array}$ & $\underset{82}{x}$ & $\begin{array}{l}1 \\
2\end{array}$ \\
\hline
\end{tabular}

Graduate nurse on duty at all times

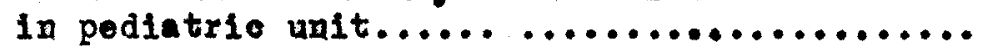

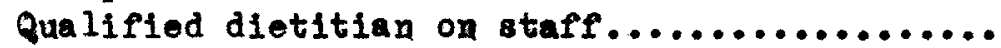
Modioal social service organized as department. Clinical labratory in hospital............... Seleoted labratory services avallable.

$\begin{array}{rrr}5 & 3 & 86 \\ 26 & 65 & X \\ 3 & 92 & X \\ 46 & 46 & X\end{array}$

(blood level for sulfonamids, sodimentation rate, blood cultures, and sorun protein). ?... Blood or plasma bank an hospital or aocessible 1 Rh nogativo blood roadily arailablel......... Oxygen tents in hospital for use with ohildren. Indiridual equipment (thermometer, towel, and wash basin) for infants and oldor ohildron.... All milk pasturized for infants and oldor childrenl..................... Isolation and/or cubicle separation prodedure followed for now admissions................

\begin{tabular}{rrrr}
32 & 11 & 18 & 2 \\
60 & 1 & $X$ & 2 \\
24 & 36 & $X$ & 3 \\
51 & 44 & $X$ & 2 \\
21 & 39 & 1 & 2 \\
55 & 4 & 1 & 3 \\
38 & 45 & 4 & 10 \\
\hline
\end{tabular}

1. Data not tabulated for hospitals with 5-24 bods. 
Table 33. Sumary of seleoted data rolated to oare of nowborn in 97 individual general hospitals in Kontuoky.

Type of service:

Floor area per bassinot in newborn nursery:
Code 1. Maternity and ohildren (with or without other adults)........

2. Materaity hospitals ............

3. Iaternity and other adults (no childron except nowborn)....

4. Podietrle hospltal..............

5. Children and adults (no maternity)

Code 1. Less than $15 \mathrm{sq}$. ft............

2. 15-24 sq. ft.................

3.25 or more sq. ft..............

4. No newborn nurgery..............

blank. Tnknown.....................

Number of beds, all patients .............................

Number of bassinots .....................................

Number of inoubators.....................................

Number of birthe during one year

Number of prematures cared for during one year. Number of hospitals reporting on prematures

1

$\ldots \ldots \ldots \ldots \ldots \ldots \ldots$

Not

6. 088

1,109

134

27,059

1,140

61

Hot

Separate nursery for nowborns only.....................

Nursery for full-time siok or suspeot separate fron woll.....

Separate nursery for promatures.

Graduate nurse on duty at a 11

times in, nowborn mursery......

Graduate murse on duty at $a 11$

times in premature nursery....

Codes

Yos $\quad$ No

applianble

(1)

(0)

(9)

$\frac{\text { roportod }}{\text { (blank) }}$

86

982

5

0

$9 \quad 87$

5

1

$49 \quad 32$

14

2

80

87

2

Runaing water for hand washing in mursery ................

Room used exolusively for pre-

paration of formulae...........

All mixtures sterilized for

newbor $\mathrm{a} \ldots \ldots \ldots \ldots \ldots \ldots \ldots \ldots$

$\begin{array}{cccc}51 & 10 & X & 2 \\ 24 & 37 & x & 2 \\ 60 & 0 & 1 & 2\end{array}$

Outpatient department admitting childrer..................

General pediatrio clinic separate from olinio for adults....

Regiatered by

Ameriona Medion 1 Association...

\begin{tabular}{rrrr}
6 & 91 & $x$ & $\times$ \\
1 & 4 & 91 & 1 \\
71 & 26 & $x$ & $\times$ \\
\hline
\end{tabular}

1. Data not tabulated for hospitals with 5-24 beds. 
Table 34, Part I. Name and looation of special hospitals in Study, by type of hospital in Kontuoky.

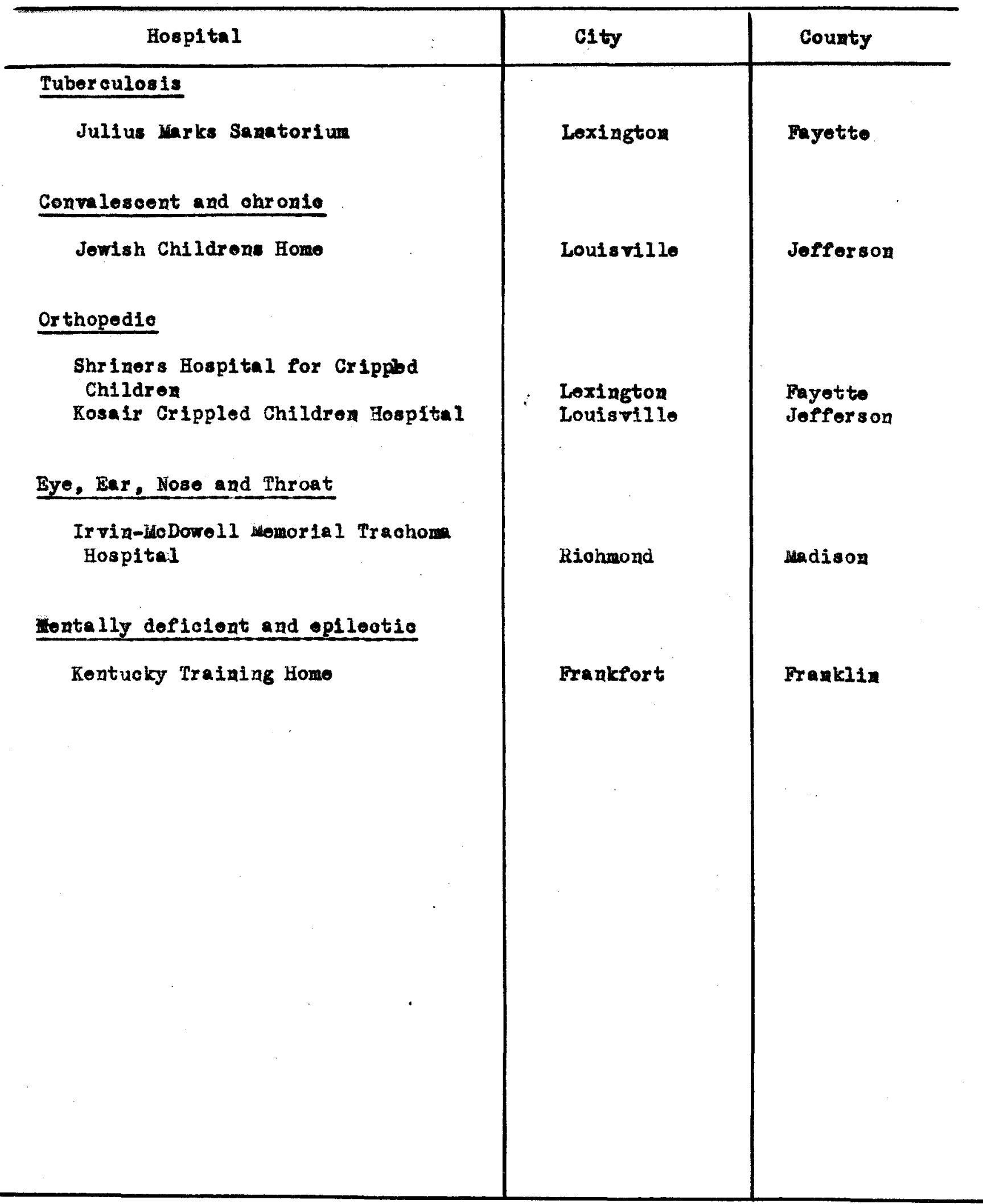


Tablo 34, Part II. Selooted data related to ohild care in indiridual speoial hospitals in Kontuoky.

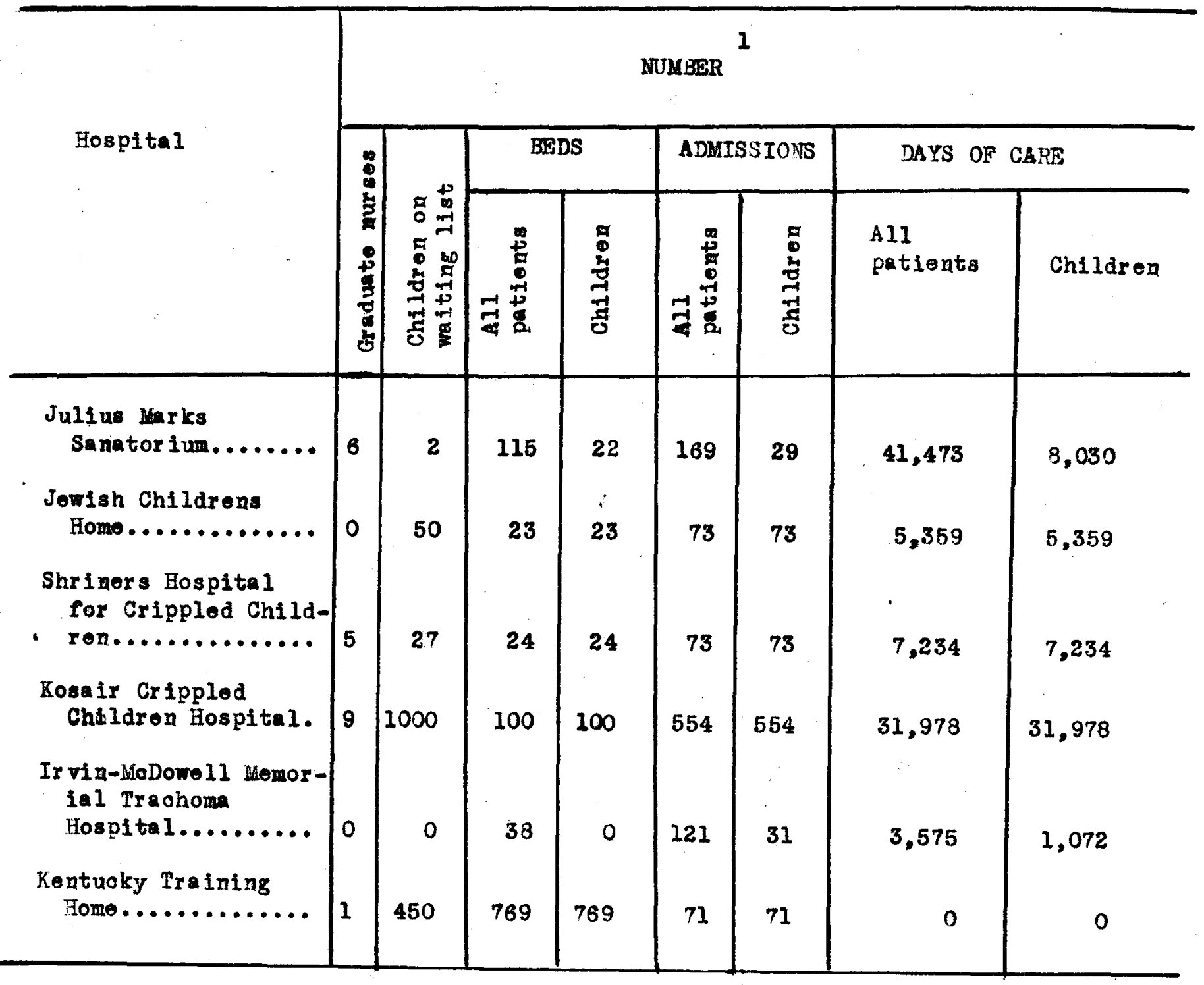

1. Blank space iadicates that speoific item was not reported. 
Table 35. Outpatient services for ohildren, by oounty group in Kentucky.

\begin{tabular}{|c|c|c|c|c|c|c|}
\hline & \multirow[b]{2}{*}{$\begin{array}{l}\text { Whole } \\
\text { State }\end{array}$} & \multicolumn{5}{|c|}{ County group } \\
\hline & & $\begin{array}{l}\text { Greater } \\
\text { motro- } \\
\text { politan }\end{array}$ & $\begin{array}{l}\text { Losser } \\
\text { metrom } \\
\text { politan }\end{array}$ & Adjacent & $\begin{array}{l}\text { Isolated } \\
\text { semi - } \\
\text { rural }\end{array}$ & $\begin{array}{l}\text { Isolated } \\
\text { rural }\end{array}$ \\
\hline 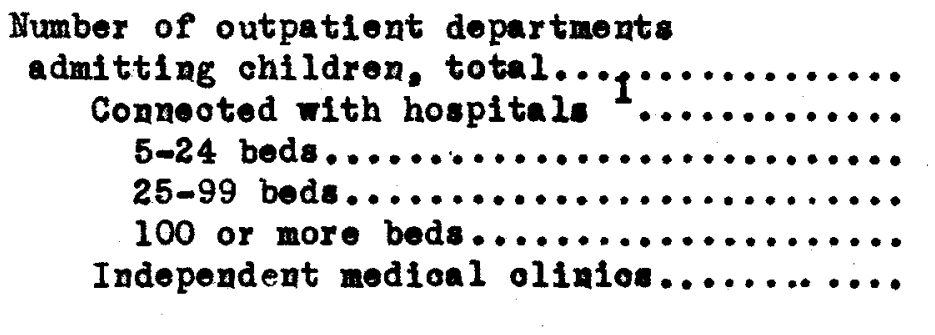 & $\begin{array}{l}6 \\
6 \\
1 \\
2 \\
3 \\
0\end{array}$ & & $\begin{array}{l}2 \\
2 \\
0 \\
0 \\
2 \\
0\end{array}$ & $\begin{array}{l}1 \\
1 \\
0 \\
1 \\
0 \\
0\end{array}$ & $\begin{array}{l}1 \\
1 \\
0 \\
0 \\
1 \\
0\end{array}$ & $\begin{array}{l}2 \\
2 \\
1 \\
1 \\
0 \\
0\end{array}$ \\
\hline 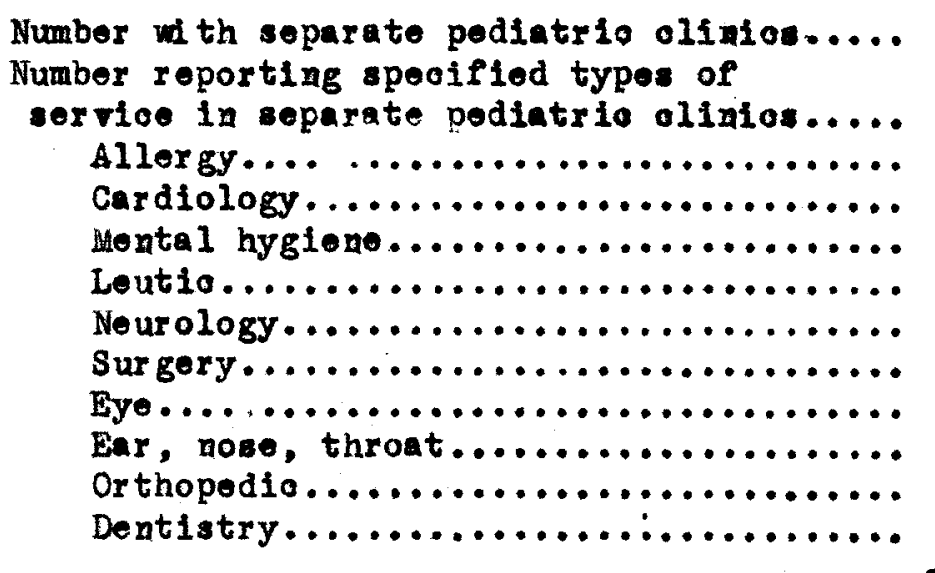 & $\begin{array}{l}1 \\
1 \\
1 \\
1 \\
1 \\
1 \\
1 \\
1 \\
1 \\
1 \\
1 \\
0\end{array}$ & & $\begin{array}{l}1 \\
1 \\
1 \\
1 \\
1 \\
1 \\
1 \\
1 \\
1 \\
1 \\
1 \\
0\end{array}$ & $\begin{array}{l}0 \\
0 \\
0 \\
0 \\
0 \\
0 \\
0 \\
0 \\
0 \\
0 \\
0 \\
0\end{array}$ & $\begin{array}{l}0 \\
0 \\
0 \\
0 \\
0 \\
0 \\
0 \\
0 \\
0 \\
0 \\
0 \\
0\end{array}$ & $\begin{array}{l}0 \\
0 \\
0 \\
0 \\
0 \\
0 \\
0 \\
0 \\
0 \\
0 \\
0 \\
0\end{array}$ \\
\hline $\begin{array}{l}\text { Number of pediatrio elinios reporting visits } \\
\text { Visits to pediatrio olialos during ono year } \\
\text { Total vislts duxing one year by ohildren } \\
\text { to outpatient departments.3.............. }\end{array}$ & 12,657 & & 8,801 & . & 1,170 & 1,981 \\
\hline
\end{tabular}

1. In non-proprietary general hospitals.

2. Question not inoluded in form used in Kentuoky.

3. When total visits by ohildren not reported they were assumed to be equal to risits to pediatrie olinios. If neither flgure was reported, total jisits by ohildren was estimated as $10 \%$ of total visits to outm patient department. 
Table 37. Community health servioes for children during one year, by oounty and in each oity of 50,000 or moro population in Kontuoky.

\begin{tabular}{|c|c|c|c|c|c|c|c|c|c|c|c|c|c|c|c|c|}
\hline \multirow{4}{*}{$\begin{array}{l}\text { County, and oity } \\
\text { of } 50,000 \text { or more } \\
\text { population }\end{array}$} & \multicolumn{4}{|c|}{$\begin{array}{l}\text { Modionl woll-ohild } \\
\text { confer enoes }\end{array}$} & \multicolumn{2}{|c|}{$\begin{array}{l}\text { Dontal } \\
\text { olinios }\end{array}$} & \multicolumn{2}{|c|}{$\begin{array}{l}\text { Publio } \\
\text { health } \\
\text { nursiag }\end{array}$} & \multicolumn{2}{|c|}{$\begin{array}{l}\text { Sohool } \\
\text { health } \\
\text { services }\end{array}$} & \multicolumn{6}{|c|}{ Location of olinios } \\
\hline & \multirow{2}{*}{\multicolumn{2}{|c|}{$\begin{array}{l}\text { Number of } \\
\text { sessions }\end{array}$}} & \multirow{2}{*}{\multicolumn{2}{|c|}{$\begin{array}{l}\text { Number of } \\
\text { conters }\end{array}$}} & \multirow{2}{*}{\multicolumn{2}{|c|}{$\begin{array}{l}\text { Number of } \\
\text { dontist- } \\
\text { hours }\end{array}$}} & \multirow{2}{*}{\multicolumn{2}{|c|}{$\begin{array}{l}\text { Number of } \\
\text { full-tine } \\
\text { publio } \\
\text { health } \\
\text { nurses }\end{array}$}} & \multirow{3}{*}{ 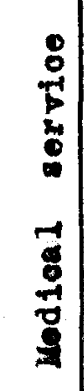 } & \multirow{3}{*}{ 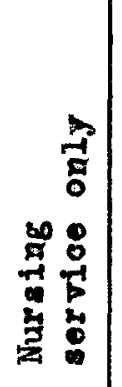 } & \multirow{3}{*}{ 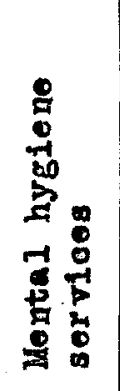 } & \multicolumn{5}{|c|}{$\begin{array}{l}\text { Sorvioes for } \\
\text { physioally-handiopped }\end{array}$} \\
\hline & & & & & & & & & & & & 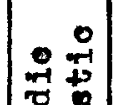 & $\underset{-1}{0}$ & & & \\
\hline & off. & Vol. & off. & Vol. & ofe. & Vol. & off. & Vol. & & & & 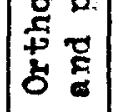 & 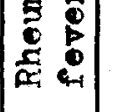 & $\begin{array}{c}0 \\
0 \\
0 \\
0 \\
2 \\
-2\end{array}$ & 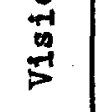 & 站 \\
\hline 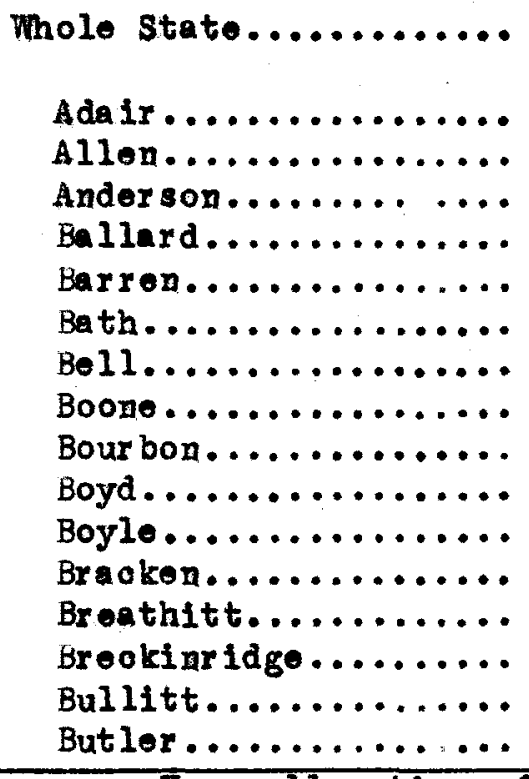 & $\begin{array}{l}14 \\
12\end{array}$ & 188 & $\begin{array}{r}1 \\
\mathrm{NR} \\
5 \\
5\end{array}$ & - & $\begin{array}{r}4739 \\
49 \\
\\
(29)\end{array}$ & 744 & $\begin{array}{l}222 \\
1 \\
1 \\
1 \\
2 \\
2 \\
3 \\
6 \\
1 \\
2 \\
1 \\
1 \\
\end{array}$ & $\cdot$ & $\begin{array}{l}- \\
* \\
* \\
* \\
* \\
* \\
* \\
* \\
* \\
* \\
* \\
*\end{array}$ & - & - & * & - & $\because$ & $\begin{array}{l}* \\
* \\
R \\
* \\
R \\
R \\
\text { R } \\
*\end{array}$ & \\
\hline $\begin{array}{r}\bar{X} \text { - allocation of } \\
\mathrm{NR} \text { - not roported } \\
\mathrm{R} \text { - in partioular } \\
\text { handicappod } \\
\text { oluded. } \\
\text { - one or more of } \\
\text { b - aursing sor vio } \\
\text { * - pecified ser } \\
\text { (blank) - speoified ser }\end{array}$ & $\operatorname{vase}$ & $\begin{array}{l}\text { odt } \\
\text { ad } t \\
\text { raos } \\
\text { lo } f \\
\text { or } \\
\text { iver }\end{array}$ & $\begin{array}{l}180 \\
\text { m nu } \\
\text { into } \\
\text { in po }\end{array}$ & $\begin{array}{l}\text { erve } \\
\text { ses } 1 \\
\text { loont } \\
\text { tiou. }\end{array}$ & $\begin{array}{l}\text { other } \\
\text { ther } \\
\text { in per } \\
\text { Iooa }\end{array}$ & $\begin{array}{l}\text { ther } \\
\text { orral } \\
\text { oount } \\
\text { ounts }\end{array}$ & $\begin{array}{l}\text { les. } \\
\text { les. } \\
\text { Lax oor } \\
\text { althou }\end{array}$ & $\begin{array}{l}\text { exami } \\
\text { irate }\end{array}$ & and & $\begin{array}{l}\text { ) and } \\
\text { es, ho }\end{array}$ & $\begin{array}{l}\operatorname{ser} \nabla \\
\operatorname{ur} 8 \text { o }\end{array}$ & $\begin{array}{l}\text { loes } f \\
\text { f whio }\end{array}$ & $\begin{array}{l}\text { for ph } \\
\text { oh are }\end{array}$ & hysio & ind & \\
\hline
\end{tabular}



Tablo 37 (oont'd), Community hoalth servioes for ohildren during one year, by oounty and in each oity
of 50,000 or more population in Kontuoky.

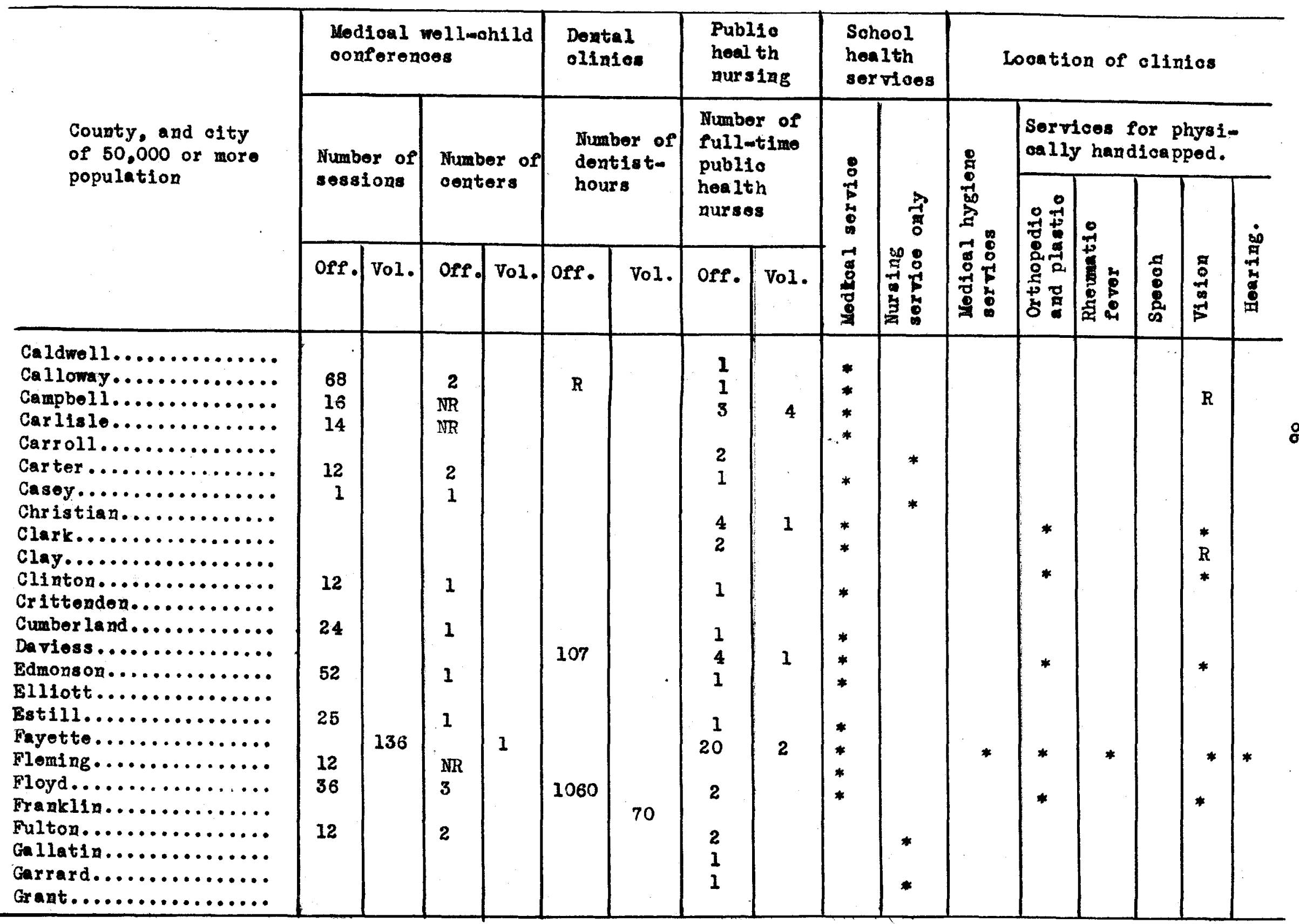


Table 37 (oont'd). Community health sorvices for ohfldron during one year, by county and in each oity
of 50,000 or more population in Kontuoky.

\begin{tabular}{|c|c|c|c|c|c|c|c|c|c|c|c|c|c|c|c|c|}
\hline \multirow{4}{*}{$\begin{array}{l}\text { County, and eity } \\
\text { of } 50,000 \text { or more } \\
\text { population }\end{array}$} & \multicolumn{4}{|c|}{$\begin{array}{l}\text { Modical wellmohild } \\
\text { oonfer enoes }\end{array}$} & \multirow{3}{*}{\multicolumn{2}{|c|}{$\begin{array}{l}\text { Doxtal } \\
\text { olinios } \\
\text { Number of } \\
\text { dontist- } \\
\text { hours }\end{array}$}} & \multirow{3}{*}{\multicolumn{2}{|c|}{$\begin{array}{l}\text { Publio } \\
\text { heal th } \\
\text { nuraing } \\
\text { Number of } \\
\text { fullutime } \\
\text { publio } \\
\text { health } \\
\text { murses }\end{array}$}} & \multicolumn{2}{|c|}{$\begin{array}{l}\text { Sohool } \\
\text { hea lth } \\
\text { servioed }\end{array}$} & \multicolumn{6}{|c|}{ Loontion of olinios } \\
\hline & \multirow{2}{*}{\multicolumn{2}{|c|}{$\begin{array}{l}\text { Nureber of } \\
\text { cessions }\end{array}$}} & \multirow{2}{*}{\multicolumn{2}{|c|}{$\begin{array}{l}\text { Number of } \\
\text { centers }\end{array}$}} & & & & & \multirow{3}{*}{ 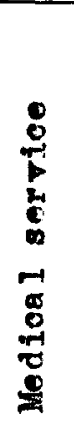 } & \multirow{3}{*}{ 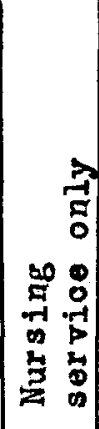 } & \multirow{3}{*}{ 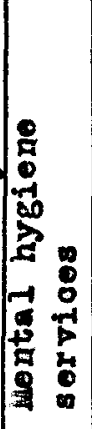 } & \multicolumn{5}{|c|}{$\begin{array}{l}\text { Servioes for physim } \\
\text { cally handicapped }\end{array}$} \\
\hline & & & & & & & & & & & & \multirow{2}{*}{ 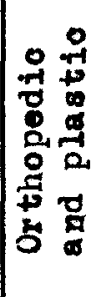 } & \multirow{2}{*}{ 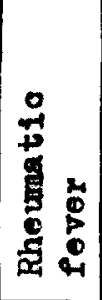 } & \multirow[b]{2}{*}{$\begin{array}{l}\text { : } \\
0 \\
0 \\
0 \\
0 \\
\text { on }\end{array}$} & \multirow[b]{2}{*}{$\begin{array}{l}5 \\
0 \\
0 \\
0 \\
0 \\
5\end{array}$} & \multirow[b]{2}{*}{ 象 } \\
\hline & off. & Vol. & off. & Vol. & Off. & Vol. & off. & Vol. & & & & & & & & \\
\hline 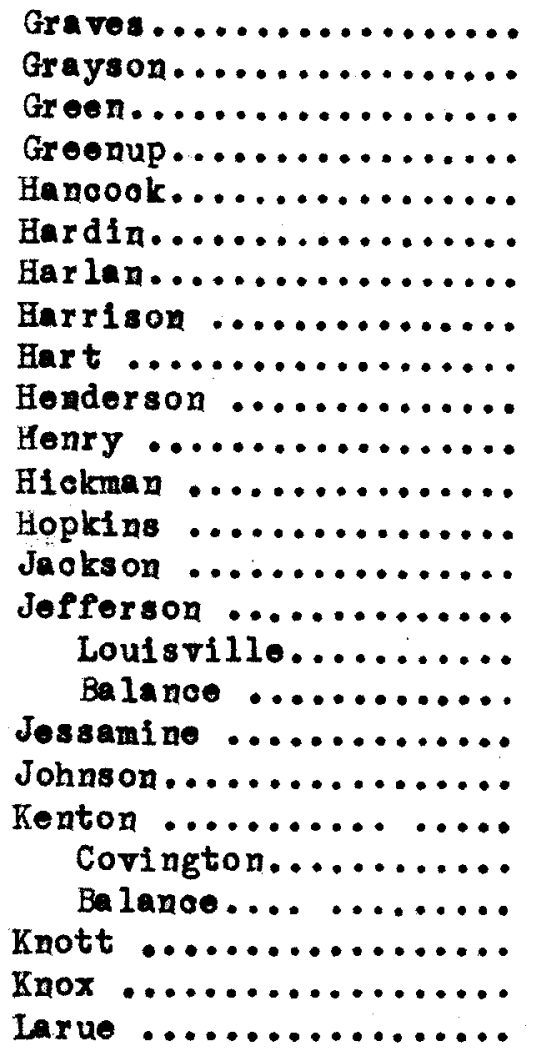 & $\begin{array}{r}29 \\
922 \\
X \\
X\end{array}$ & & $\begin{array}{c}3 \\
30 \\
10 \\
42 \\
\mathrm{NR} \\
\mathrm{NR}\end{array}$ & & $\begin{array}{r}2343 \\
X \\
X\end{array}$ & 452 & $\begin{array}{c}2 \\
1 \\
1 \\
2 \\
1 \\
4 \\
2 \\
3 \\
1 \\
5 \\
\\
1 \\
\\
53 \\
X \\
X \\
\\
2 \\
5 \\
X \\
X \\
1 \\
1\end{array}$ & $\begin{array}{r}1 \\
21 \\
X \\
X \\
1\end{array}$ & $\begin{array}{l}\ddots \\
* \\
* \\
* \\
* \\
* \\
* \\
* \\
* \\
* \\
* \\
* \\
* \\
* \\
* \\
*\end{array}$ & * & * & $\begin{array}{l}* \\
* \\
*\end{array}$ & & & $\begin{array}{l}\mathrm{R} \\
\mathrm{R} \\
\\
* \\
\mathrm{R} \\
*\end{array}$ & \\
\hline
\end{tabular}



Table 37 (cont'd). Communty health sorvices for children during one year, by oounty and in oach
city of 60,000 or more population in Kentuoky.

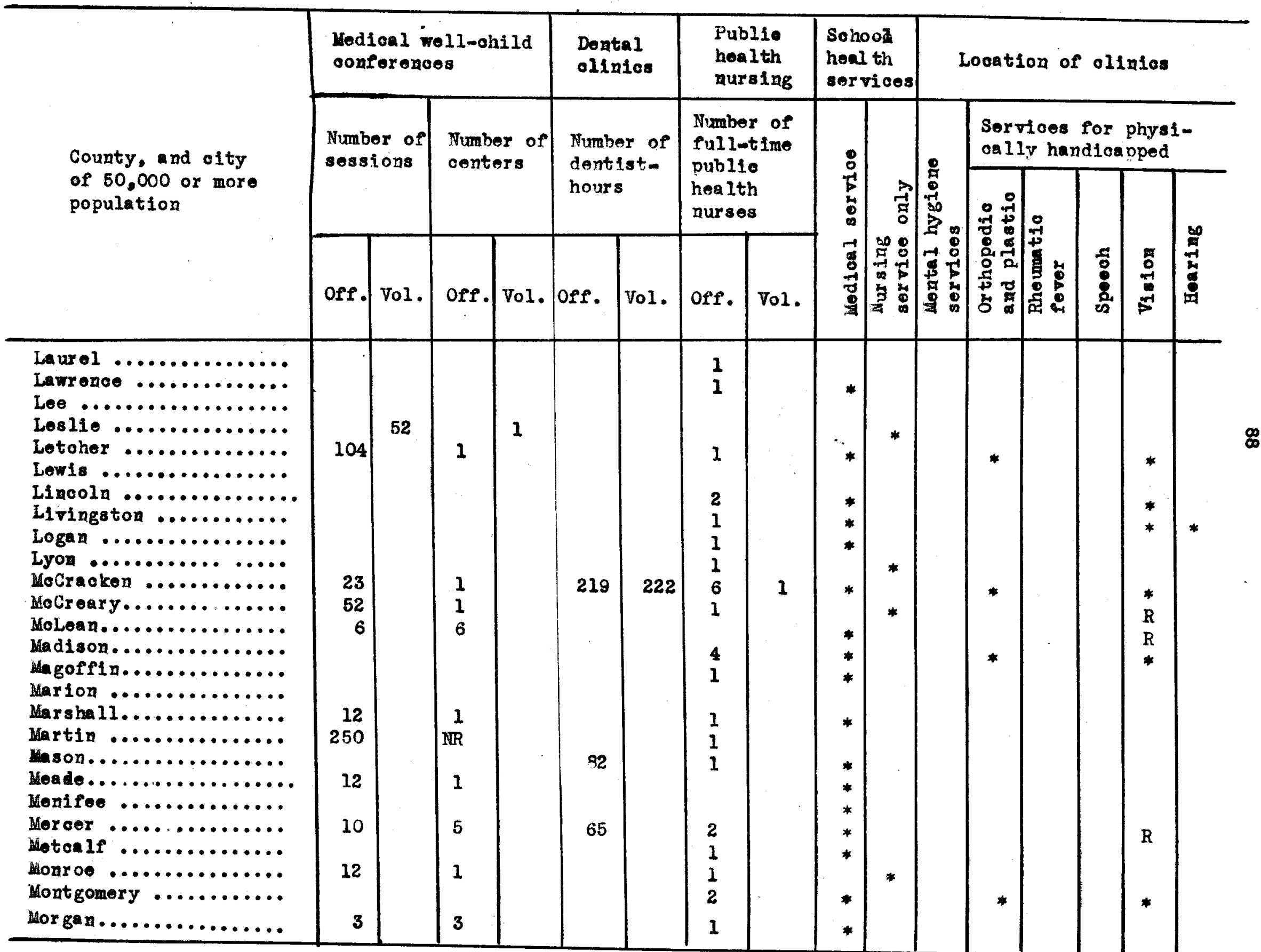


Table 37 (cont'd). Comnunity health sorvices for ohildren during one year, by oounty and in ea oh oity
of 50,000 or nore population in Kontuoky.

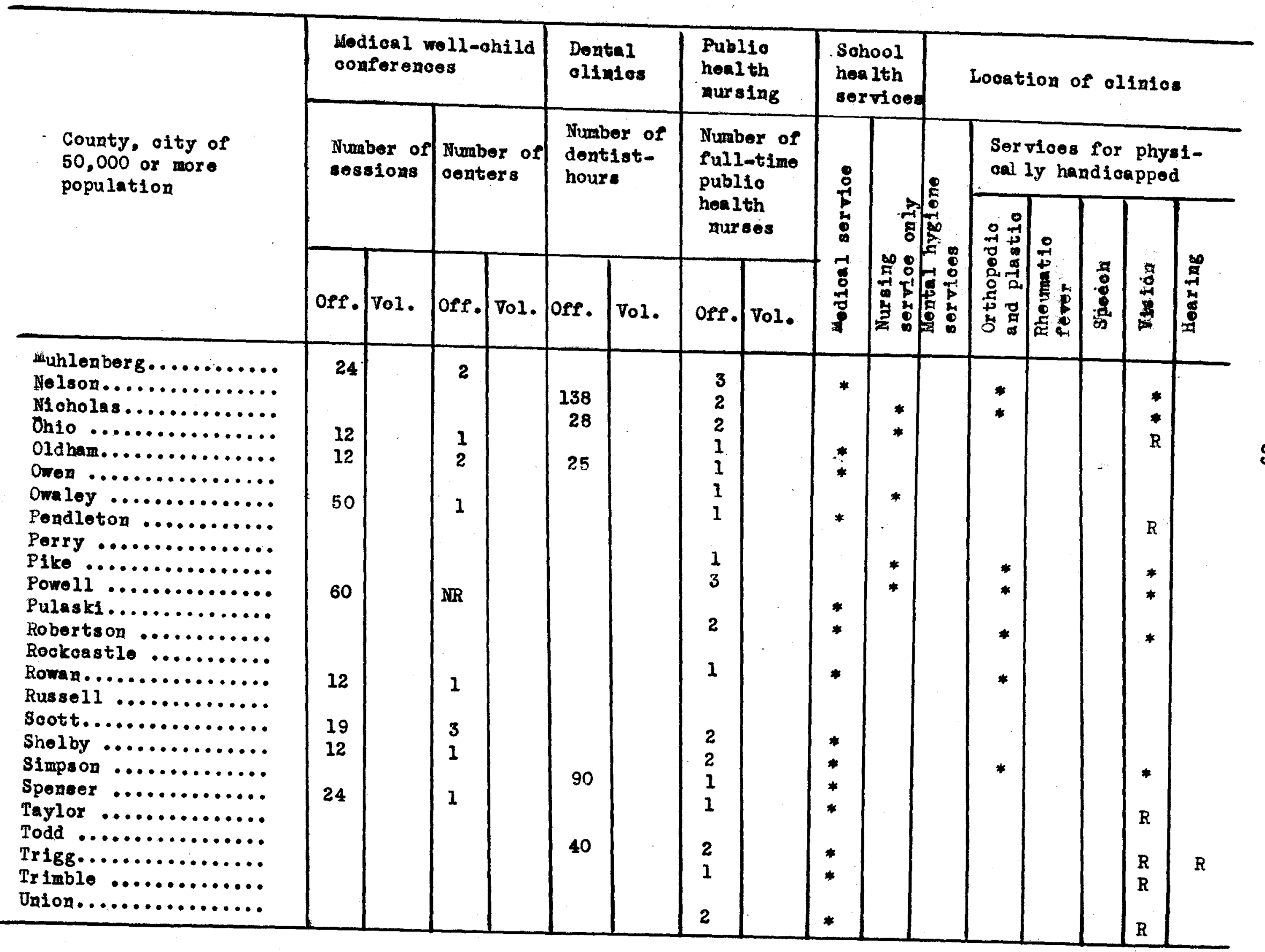


Table 37 (oont'd). Conenuity heal th servioes for ohildren during one year by county and in each ofty
of 50,000 or more population in Kentuoky.

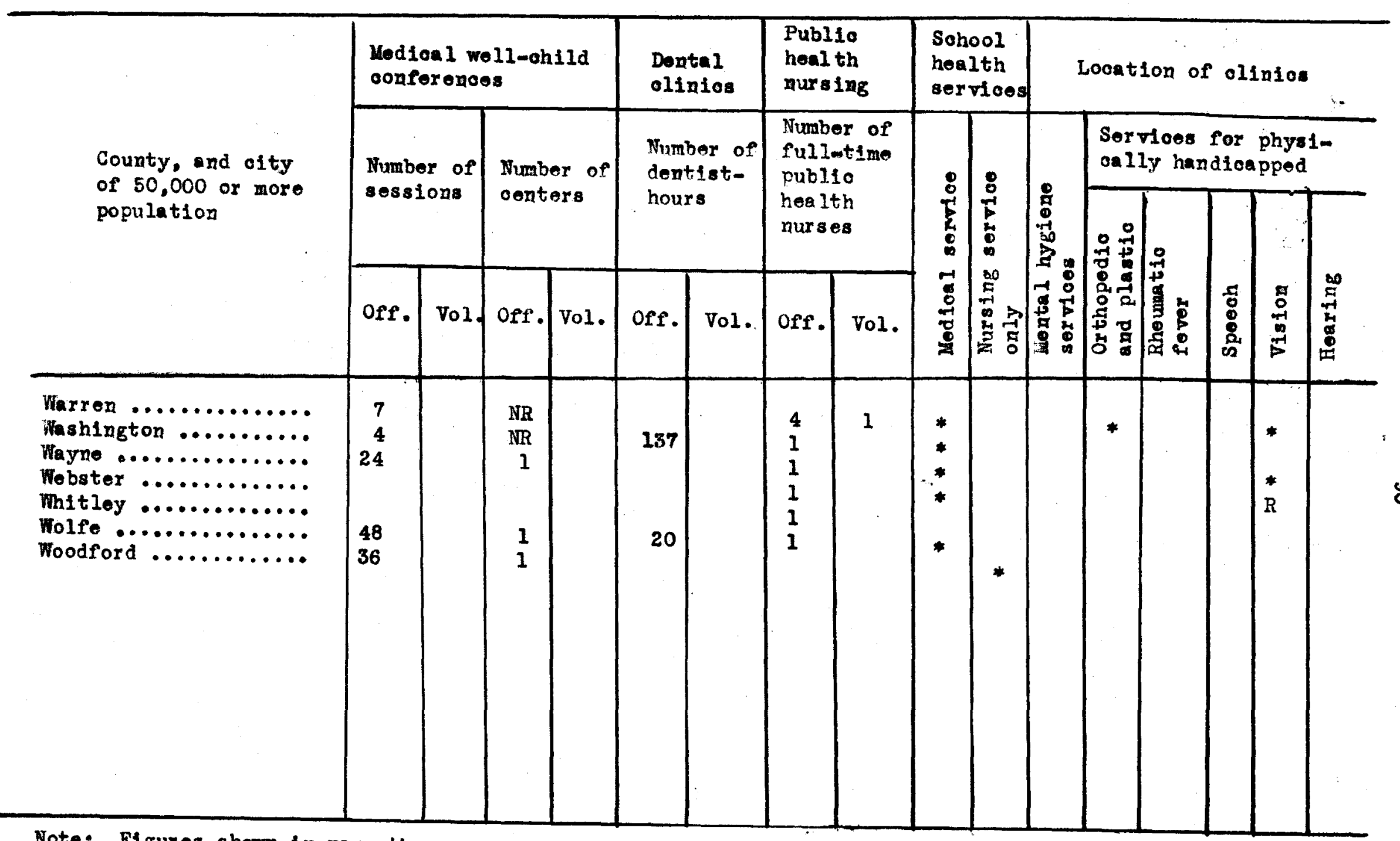

Note: Figures shown in parentheses are statistical estimatos. 
Pable 38. Sessions of medical well-child conferences during one year, by county gooup in Kontucky

\begin{tabular}{|c|c|c|c|c|c|c|}
\hline \multirow[b]{2}{*}{ County group } & \multirow[b]{2}{*}{ Agonoy } & \multicolumn{4}{|c|}{ Number of sessions } & \multirow{2}{*}{$\begin{array}{l}\text { Number of } \\
\text { sossions } \\
\text { per } 1,000 \\
\text { ohildren } \\
\text { under } 5\end{array}$} \\
\hline & & Total & $\begin{array}{l}\text { Infaute } \\
\text { only }\end{array}$ & $\begin{array}{l}\text { Presohool } \\
\text { ohildren } \\
\text { only }\end{array}$ & $\begin{array}{l}\text { Infants and } \\
\text { prosohool } \\
\text { children }\end{array}$ & \\
\hline Whole state...$\ldots \ldots \ldots$ & $\begin{array}{l}\text { All } \\
\text { Official } \\
\text { Voluntary }\end{array}$ & $\begin{array}{r}2,699 \\
2,511 \\
188\end{array}$ & $\begin{array}{l}56 \\
30 \\
26\end{array}$ & $\begin{array}{l}62 \\
36 \\
26\end{array}$ & $\begin{array}{r}2,581 \\
2,445 \\
136\end{array}$ & $\begin{array}{l}7.8 \\
7.3 \\
0.5\end{array}$ \\
\hline Oreater Metropolitan.. & $\begin{array}{l}\text { All } \\
\text { Offiolal } \\
\text { Voluntaxy }\end{array}$ & & & $\because$ & & \\
\hline Lesser notropolitan... & $\begin{array}{l}\text { All } \\
\text { Offloial } \\
\text { Voluntary }\end{array}$ & $\begin{array}{c}1,035 \\
1,035 \\
0\end{array}$ & $\begin{array}{l}0 \\
0 \\
0\end{array}$ & $\begin{array}{l}0 \\
0 \\
0\end{array}$ & $\begin{array}{r}1,035 \\
1,035 \\
0\end{array}$ & $\begin{array}{r}15.4 \\
15.4 \\
-.\end{array}$ \\
\hline Adjacent............ & $\begin{array}{l}\text { All } \\
\text { Official } \\
\text { Voluntary }\end{array}$ & $\begin{array}{r}71 \\
71 \\
0\end{array}$ & $\begin{array}{l}0 \\
0 \\
0\end{array}$ & $\begin{array}{l}0 \\
0 \\
0\end{array}$ & $\begin{array}{r}71 \\
71 \\
0\end{array}$ & $\begin{array}{l}2.6 \\
2.6 \\
--\end{array}$ \\
\hline Isolated semi-rural... & $\begin{array}{l}\text { All } \\
\text { Offloial } \\
\text { Voluntary }\end{array}$ & $\begin{array}{l}589 \\
453 \\
136\end{array}$ & $\begin{array}{l}0 \\
0 \\
0\end{array}$ & $\begin{array}{l}0 \\
0 \\
0\end{array}$ & $\begin{array}{l}589 \\
453 \\
136\end{array}$ & $\begin{array}{l}4.5 \\
3.5 \\
1.0\end{array}$ \\
\hline Isolated rural....... & $\begin{array}{l}\text { All } \\
\text { Offioial } \\
\text { Voluntary }\end{array}$ & $\begin{array}{r}1,004 \\
952 \\
52\end{array}$ & $\begin{array}{l}56 \\
30 \\
26\end{array}$ & $\begin{array}{l}62 \\
36 \\
26\end{array}$ & $\begin{array}{r}886 \\
886 \\
0\end{array}$ & $\begin{array}{l}8.3 \\
7.9 \\
0.4\end{array}$ \\
\hline
\end{tabular}


Table 39. Patients and visits to modical woll-ohild conferences during one year, by oounty group in Kentucky

\begin{tabular}{|c|c|c|c|c|c|c|c|c|c|c|c|c|c|c|}
\hline \multirow[b]{2}{*}{ County group } & \multirow{2}{*}{ Agenoy } & \multicolumn{4}{|c|}{ Number of patieats } & \multicolumn{4}{|c|}{ Number of visits } & \multicolumn{2}{|c|}{$\begin{array}{l}\text { Number } \\
\text { per } 1000 \\
\text { ohildr on } \\
\text { under } \\
5\end{array}$} & \multicolumn{3}{|c|}{$\begin{array}{l}\text { Number of } \\
\text { visits por } \\
\text { patient }\end{array}$} \\
\hline & & $\begin{array}{l}\overrightarrow{1} \\
\overrightarrow{5} \\
\overrightarrow{0}\end{array}$ & 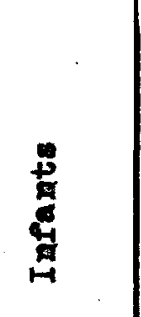 & $\begin{array}{c}-1 \\
0 \\
0 \\
0 \\
0 \\
0 \\
0 \\
0\end{array}$ & 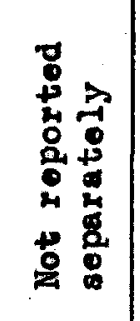 & $\underset{\$}{+1}$ & $\underset{⿱ 中}{\stackrel{D}{*}}$ & $\begin{array}{l}-1 \\
0 \\
0 \\
0 \\
0 \\
0 \\
0 \\
2\end{array}$ & 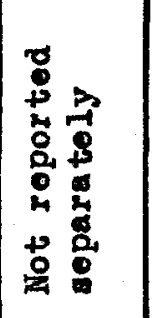 & | & 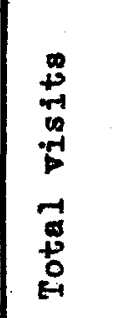 & 焉 & 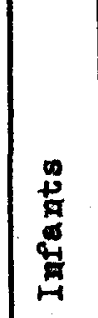 & $\begin{array}{l}0 \\
0 \\
0 \\
\frac{5}{0} \\
0 \\
0 \\
0 \\
1\end{array}$ \\
\hline $\begin{array}{l}\text { Whole state......... } \\
\text { Greater motropolitan }\end{array}$ & $\begin{array}{l}\text { All } \\
\text { Offioial } \\
\text { Voluntary } \\
\text { All } \\
\text { Official } \\
\text { Voluntary }\end{array}$ & $\begin{array}{r}17,996 \\
17,421 \\
575\end{array}$ & $\begin{array}{r}6,697 \\
6,222 \\
475\end{array}$ & $\begin{array}{r}9,919 \\
9,819 \\
100\end{array}$ & $\begin{array}{r}1,390 \\
1,380 \\
0\end{array}$ & $\begin{array}{r}32,774 \\
31,906 \\
868\end{array}$ & $\begin{array}{r}18,090 \\
12.482 \\
608\end{array}$ & $\begin{array}{r}17.678 \\
17.418 \\
260\end{array}$ & $\begin{array}{r}2,009 \\
2,009 \\
0\end{array}$ & $\mid \begin{array}{r}51.9 \\
50.2 \\
1.7\end{array}$ & $\begin{array}{r}94.6 \\
92.1 \\
2.5\end{array}$ & $\begin{array}{l}1.8 \\
1.8 \\
1.5\end{array}$ & $\begin{array}{l}2.0 \\
2.0 \\
1.3\end{array} \mid$ & $\begin{array}{l}1.8 \\
1.8 \\
2.6\end{array}$ \\
\hline Lesser metropolitan. & $\begin{array}{l}\text { Al1 } \\
\text { Offieial } \\
\text { Voluntary }\end{array}$ & $\begin{array}{r}7,979 \\
8,979 \\
0\end{array}$ & $\begin{array}{r}3,060 \\
3,060 \\
0\end{array}$ & $\begin{array}{r}4,731 \\
4,731 \\
0\end{array}$ & $\begin{array}{r}188 \\
188 \\
0\end{array}$ & $\begin{array}{r}16,471 \\
16,471 \\
0\end{array}$ & $\begin{array}{r}6.928 \\
6,928 \\
0\end{array}$ & $\begin{array}{r}9,290 \\
9,290 \\
0\end{array}$ & $\begin{array}{r}253 \\
253 \\
0\end{array}$ & $\begin{array}{c}119.0 \\
119.0 \\
-\end{array}$ & $\begin{array}{r}245.6 \\
245.6 \\
--\end{array}$ & $\begin{array}{l}2.1 \\
2.1 \\
-.\end{array}$ & $\mid \begin{array}{l}2.3 \\
2.3 \\
--\end{array}$ & $\begin{array}{l}2.0 \\
2.0 \\
--\end{array}$ \\
\hline Adjacent............ & $\begin{array}{l}\text { All } \\
\text { Official } \\
\text { Voluntary }\end{array}$ & $\begin{array}{r}664 \\
664 \\
0\end{array}$ & $\begin{array}{r}234 \\
234 \\
0\end{array}$ & $\begin{array}{r}165 \\
165 \\
0\end{array}$ & $\begin{array}{r}265 \\
265 \\
0\end{array}$ & $\begin{array}{r}954 \\
954 \\
0\end{array}$ & $\begin{array}{r}306 \\
306 \\
0\end{array}$ & $\begin{array}{r}260 \\
260 \\
0\end{array}$ & $\begin{array}{r}388 \\
388 \\
0\end{array}$ & $\begin{array}{c}24.1 \\
24.1 \\
-\end{array} \mid$ & $\begin{array}{r}34.6 \\
34.6 \\
-.\end{array}$ & $\mid \begin{array}{l}1.4 \\
1.4 \\
-\end{array}$ & $\mid-$ & $\begin{array}{l}-- \\
-- \\
-\end{array}$ \\
\hline Isolated semi-rural. & $\begin{array}{l}\text { All } \\
\text { Offioial } \\
\text { Voluntary }\end{array}$ & $\begin{array}{r}2.722 \\
2.222 \\
500\end{array}$ & $\begin{array}{r}1,079 \\
\quad 629 \\
450\end{array}$ & $\begin{array}{r}1,550 \\
1,500 \\
50\end{array}$ & $\begin{array}{l}93 \\
93 \\
0\end{array}$ & $\begin{array}{r}4,342 \\
3,724 \\
618\end{array}$ & $\begin{array}{r}1,640 \\
1,132 \\
508\end{array}$ & $\begin{array}{r}2,357 \\
2,247 \\
110\end{array}$ & $\begin{array}{r}345 \\
345 \\
0\end{array}$ & $\begin{array}{r}20.7 \\
16.9 \\
3.8\end{array}$ & $\begin{array}{r}33.1 \\
28.4 \\
4.7\end{array}$ & $\begin{array}{l}1.5 \\
1.7 \\
1.2\end{array}$ & $\begin{array}{l}1.5 \\
1.8 \\
1.1\end{array}$ & $\begin{array}{l}1.5 \\
1.5 \\
2.2\end{array}$ \\
\hline Isolated rural...... & $\begin{array}{l}\text { All } \\
\text { Offloial } \\
\text { Voluntary }\end{array}$ & $\begin{array}{r}6,631 \\
6,556 \\
75\end{array}$ & $\begin{array}{r}2,324 \\
2,299 \\
25\end{array}$ & $\begin{array}{r}3,473 \\
3,423 \\
50\end{array}$ & $\begin{array}{r}834 \\
834 \\
0\end{array}$ & $\begin{array}{r}11,007 \\
10,757 \\
250\end{array}$ & $\begin{array}{r}4,216 \\
4,116 \\
100\end{array}$ & $\begin{array}{r}5,771 \\
5,621 \\
150\end{array}$ & $\begin{array}{r}1,02 d \\
1,02 q\end{array}$ & $\begin{array}{r}59.9 \\
54.3 \\
0.6\end{array}$ & $\begin{array}{r}91.2 \\
89.1 \\
2.1\end{array}$ & $\begin{array}{l}1.7 \\
1.6 \\
3.3\end{array}$ & $\mid--$ & $\begin{array}{l}= \\
-- \\
3,0\end{array}$ \\
\hline
\end{tabular}


Table 40. Immunizations given routinoly in nodical well-ohild oonferenoes during one yoar, by
county group in Kontuoky.

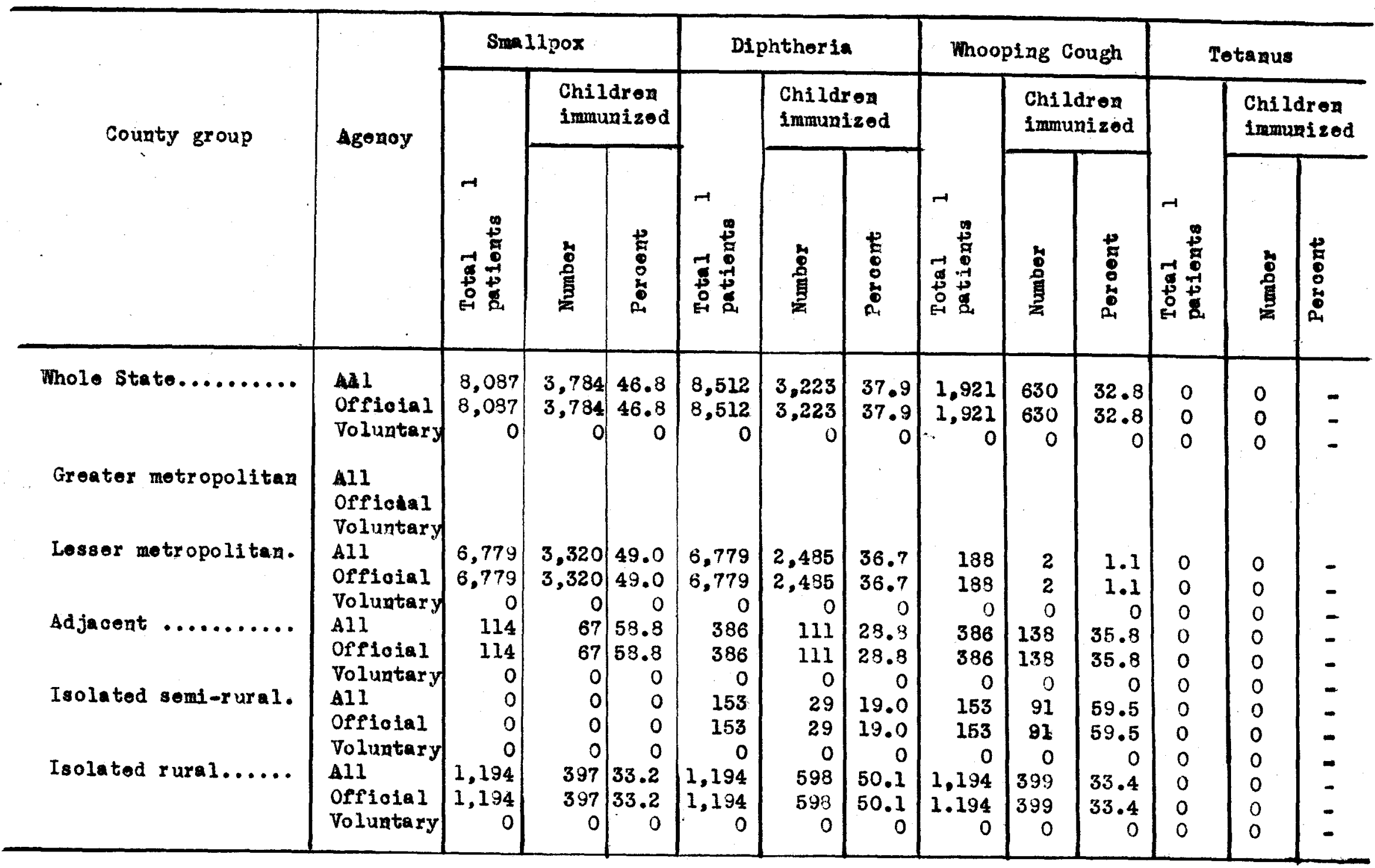

1. Patients in conferenoes in which specified immunieation is performed routinely and number immunized if known 
Table 4l. Staff of medioal well-ohild oonferenoes, by oounty group in Kentuoky.

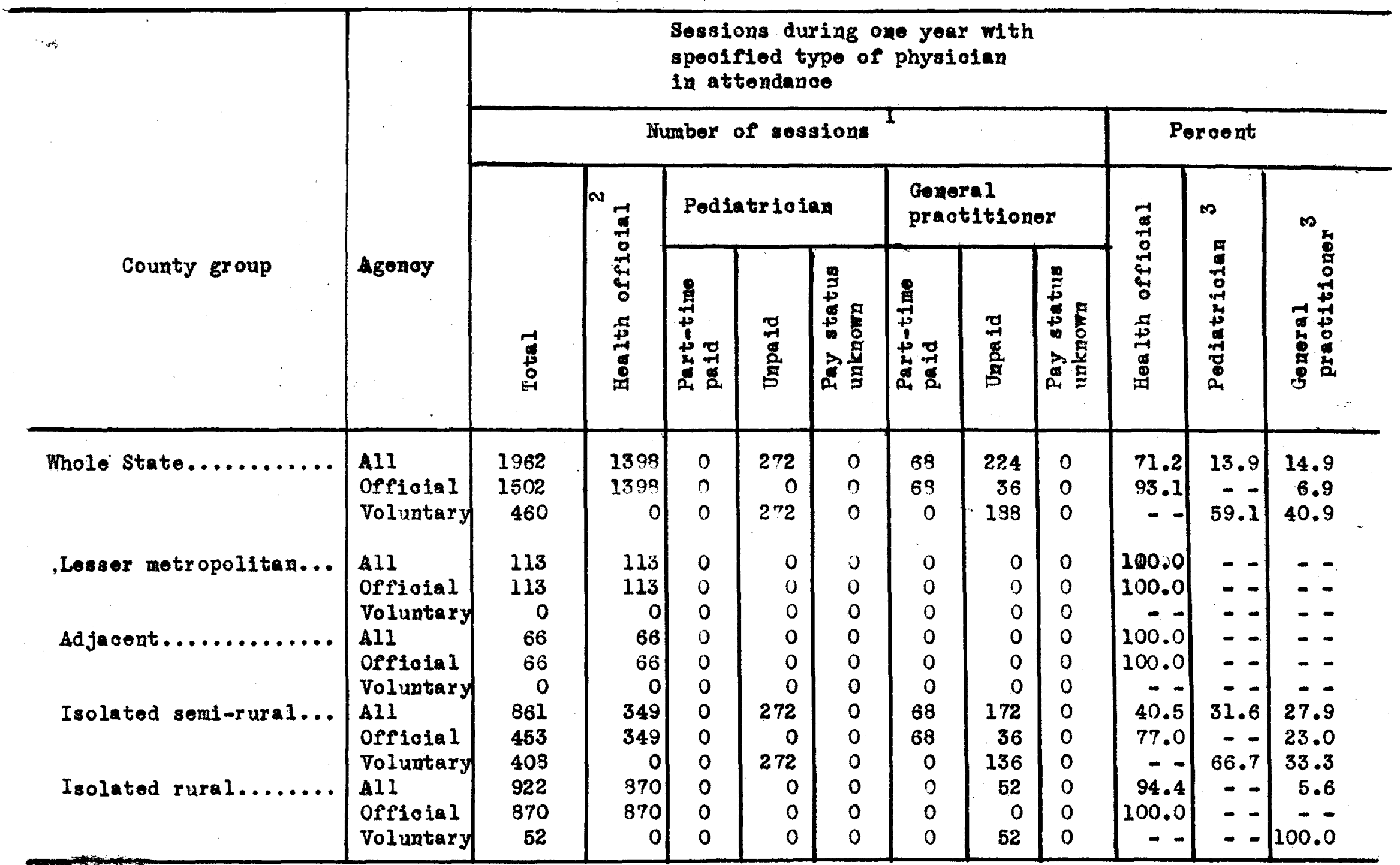

1. Wach session is counted onoe for each physioian in attendance. Col (1) is sum of cols. (2) - (8). 2. Includes health offioers, all full-time pald physioians and hospital house staff.

3. Col. (10) is sum of cols. (3), (4) and (5) divided by ool. (1). Sinilar prooodure for ool. (11). Notes Usual time per session was two or more hours for 100 per oont of the sessions reporting this item. 
Table:42. Practioes in medionl woll-ohild oonforenoes, by county groul in Kentuoky.

\begin{tabular}{|c|c|c|c|c|c|c|c|c|c|c|c|c|c|c|c|c|}
\hline \multirow{5}{*}{ County Group } & \multirow{5}{*}{ Agenoy } & \multicolumn{5}{|c|}{ Routino immunizations } & \multicolumn{3}{|c|}{$\begin{array}{l}\text { Publite hoalth } \\
\text { nursiag follow- } \\
\text { up in the howe }\end{array}$} & \multicolumn{7}{|c|}{ Other Sorvices } \\
\hline & & \multirow{4}{*}{ 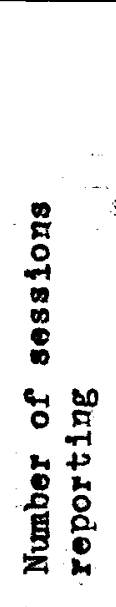 } & \multicolumn{4}{|c|}{$\begin{array}{l}\text { Sessions giring } \\
\text { sorvioo }\end{array}$} & \multirow{4}{*}{ 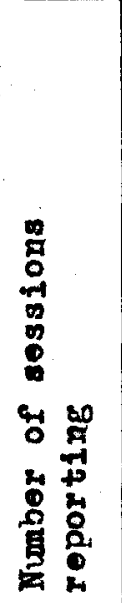 } & \multirow{3}{*}{\multicolumn{2}{|c|}{$\begin{array}{l}\text { Sessions } \\
\text { giving } \\
\text { servioe }\end{array}$}} & \multirow{4}{*}{ 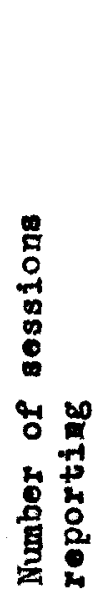 } & \multicolumn{6}{|c|}{ Sessions giving servioe } \\
\hline & & & \multirow{2}{*}{\multicolumn{2}{|c|}{$\begin{array}{l}\text { Sma11pox } \\
\text { and } \\
\text { diphtheria }\end{array}$}} & \multirow{2}{*}{\multicolumn{2}{|c|}{$\begin{array}{l}\text { Whooping } \\
\text { Cough }\end{array}$}} & & & & & \multirow{2}{*}{\multicolumn{2}{|c|}{$\begin{array}{l}\text { Advioe to } \\
\text { mothers }\end{array}$}} & \multicolumn{4}{|c|}{$\begin{array}{l}\text { Consultant service }{ }^{2} \\
\text { by - }\end{array}$} \\
\hline & & & & & & & & & & & & & \multicolumn{2}{|c|}{$\begin{array}{l}\text { Nutri- } \\
\text { tionist }\end{array}$} & \multicolumn{2}{|c|}{$\begin{array}{l}\text { Peychologi } \\
\text { glst or } \\
\text { paychia- } \\
\text { trist }\end{array}$} \\
\hline & & & 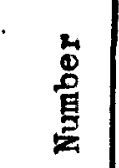 & $\begin{array}{l}+ \\
\text { 品 } \\
0 \\
0 \\
0.1 \\
0 \\
2\end{array}$ & 兽 & 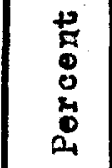 & & $\frac{4}{8}$ & 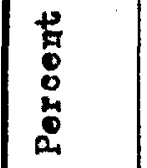 & & 葛 & $\begin{array}{l}+ \\
0 \\
0 \\
0 \\
0 \\
0\end{array}$ & $\stackrel{\mathscr{E}}{\underline{E}}$ & $\begin{array}{l}2 \\
0 \\
0 \\
0 \\
0 \\
0 \\
0.1\end{array}$ & 兽 & $\begin{array}{l}5 \\
0 \\
0 \\
0 \\
0\end{array}$ \\
\hline Whole state.. & $\begin{array}{l}\text { AlI } \\
\text { Offiolal } \\
\text { Voluntary }\end{array}$ & $\begin{array}{r}2614 \\
2426 \\
188\end{array}$ & $\begin{array}{r}2130 \\
2128 \\
52\end{array}$ & $\begin{array}{l}83.4 \\
87.7 \\
27.7\end{array}$ & $\begin{array}{r}1057 \\
1057 \\
0\end{array}$ & $\begin{array}{r}40.4 \\
43.6 \\
--\end{array}$ & $\begin{array}{r}2636 \\
2448 \\
188\end{array}$ & $\begin{array}{r}2065 \\
1877 \\
188\end{array}$ & $\begin{array}{l}78.3 \\
76.2 \\
100\end{array}$ & $\begin{array}{r}2672 \\
2484 \\
188\end{array}$ & $\begin{array}{r}2387 \\
2199 \\
188\end{array}$ & $\begin{array}{r}89.3 \\
88.5 \\
100.0\end{array}$ & $\begin{array}{r}1628 \\
1628 \\
0\end{array}$ & $\begin{array}{c}60.9 \\
65.5 \\
--\end{array}$ & $\begin{array}{r}1146 \\
1010 \\
136\end{array}$ & $\begin{array}{l}42.9 \\
40.7 \\
72.3\end{array}$ \\
\hline $\begin{array}{l}\text { Lesser met- } \\
\text { ropolitan. } \\
\text { Adjacent.... }\end{array}$ & $\begin{array}{l}\text { All } \\
\text { Offioial } \\
\text { Voluntary } \\
\text { All } \\
\text { Offloial } \\
\text { Voluntary } \\
\text { All } \\
\text { Offloial } \\
\text { Voluntary } \\
\text { All } \\
\text { Offloial } \\
\text { Voluntary }\end{array}$ & $\begin{array}{r}1035 \\
1035 \\
0 \\
60 \\
60 \\
0 \\
577 \\
441 \\
136 \\
942 \\
890 \\
52 \\
\end{array}$ & $\begin{array}{r}2035 \\
1035 \\
0 \\
48 \\
48 \\
0 \\
405 \\
405 \\
0 \\
692 \\
640 \\
52 \\
\end{array}$ & $\begin{array}{c}100.0 \\
100.0 \\
- \\
80.0 \\
80.0 \\
- \\
70.2 \\
91.8 \\
- \\
73.5 \\
71.9 \\
100.0 \\
\end{array}$ & $\begin{array}{r}28 \\
28 \\
0 \\
48 \\
48 \\
0 \\
405 \\
405 \\
0 \\
576 \\
576 \\
0\end{array}$ & $\begin{array}{c}2.7 \\
2.7 \\
- \\
80.0 \\
80.0 \\
- \\
70.2 \\
91.8 \\
- \\
61.1 \\
64.7 \\
-\end{array}$ & $\begin{array}{r}1035 \\
1035 \\
0 \\
66 \\
66 \\
0 \\
\\
553 \\
417 \\
136 \\
\\
982 \\
930 \\
52 \\
\end{array}$ & $\begin{array}{r}950 \\
950 \\
0 \\
66 \\
66 \\
0 \\
\\
553 \\
417 \\
136 \\
\\
496 \\
444 \\
52 \\
\end{array}$ & $\begin{array}{c}91.8 \\
91.8 \\
- \\
100.0 \\
100.0 \\
- \\
100.0 \\
100.0 \\
100.0 \\
50.5 \\
47.7 \\
100.0\end{array}$ & $\begin{array}{r}1035 \\
1035 \\
0 \\
66 \\
66 \\
0 \\
\\
589 \\
453 \\
136 \\
\\
982 \\
930 \\
52 \\
\end{array}$ & $\begin{array}{r}1035 \\
1035 \\
0 \\
66 \\
66 \\
0 \\
554 \\
418 \\
136 \\
732 \\
680 \\
52 \\
\end{array}$ & $\begin{array}{c}100.0 \\
100.0 \\
- \\
100.0 \\
100.0 \\
- \\
92.1 \\
92.3 \\
100.0 \\
74.5 \\
13.1 \\
100.0\end{array}$ & $\begin{array}{r}934 \\
934 \\
0 \\
42 \\
42 \\
0 \\
\\
166 \\
166 \\
0\end{array}$ & $\begin{array}{c}90.2 \\
90.2 \\
- \\
63.6 \\
63.6 \\
- \\
28.2 \\
36.6 \\
- \\
49.5 \\
52.3 \\
- \\
\end{array}$ & \begin{tabular}{r|}
922 \\
922 \\
0 \\
0 \\
0 \\
0 \\
\\
160 \\
24 \\
136 \\
\\
64 \\
64 \\
0 \\
\end{tabular} & $\begin{array}{c}89.1 \\
89.1 \\
- \\
- \\
- \\
- \\
27.2 \\
5.3 \\
100.0 \\
6.5 \\
6.9 \\
- \\
\end{array}$ \\
\hline
\end{tabular}

1. Advioe on formulae, foeding, oare and training.

2. Consultant servioe for staff and/or parents. 
Tablo 43. Pationts and visits to ohildrex's dental olinios during one year, by oounty group in Kontuoky.

\begin{tabular}{|c|c|c|c|c|c|c|c|c|c|c|c|}
\hline \multirow[b]{2}{*}{ County group } & \multirow[b]{2}{*}{ Agonoy } & \multirow[b]{2}{*}{ 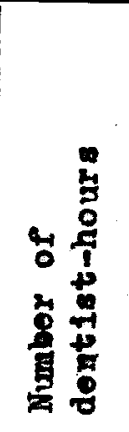 } & \multicolumn{4}{|c|}{ Number of pationts } & \multicolumn{4}{|c|}{ Number of visits } & \multirow{2}{*}{ 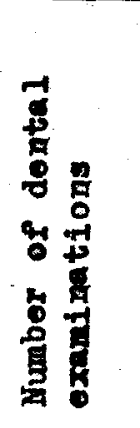 } \\
\hline & & & 吾 & $\begin{array}{l}-1 \\
0 \\
0 \\
0 \\
0 \\
0 \\
0 \\
0.1\end{array}$ & $\begin{array}{l}-1 \\
8 \\
0 \\
\frac{1}{0} \\
0\end{array}$ & 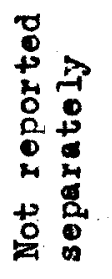 & 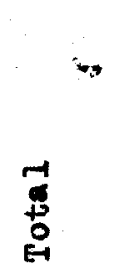 & $\begin{array}{l}\dot{1} \\
0 \\
0 \\
0 \\
0 \\
0 \\
0 \\
0 \\
14\end{array}$ & $\begin{array}{l}\overrightarrow{1} \\
: \\
\dot{c} \\
\dot{0}\end{array}$ & 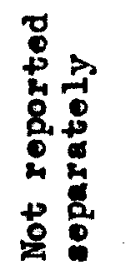 & \\
\hline Whole state....... & $\begin{array}{l}\text { All } \\
\text { Offlotal } \\
\text { Voluntary }\end{array}$ & $\begin{array}{r}5.483 \\
4.739 \\
744\end{array}$ & $\begin{array}{l}4,469 \\
3,186 \\
1,283\end{array}$ & $\begin{array}{r}12 \\
12 \\
0\end{array}$ & $\begin{array}{l}3,928 \\
2,720 \\
1,208\end{array}$ & $\begin{array}{r}529 \\
454 \\
75\end{array}$ & $\begin{array}{l}8,094 \\
6,796 \\
1,298\end{array}$ & $\begin{array}{r}18 \\
18 \\
0\end{array}$ & $\begin{array}{l}5,322 \\
4,114 \\
1,208\end{array}$ & $\begin{array}{r}2,754 \\
2,664 \\
90\end{array}$ & $\begin{array}{r}45,989 \\
40,924 \\
5,065\end{array}$ \\
\hline $\begin{array}{l}\text { Lesser motro- } \\
\text { politan........ }\end{array}$ & $\begin{array}{l}\text { All } \\
\text { Offioial } \\
\text { Voluntary }\end{array}$ & $\begin{array}{r}2,443 \\
2,443 \\
0\end{array}$ & $\begin{array}{r}552 \\
552 \\
0\end{array}$ & $\begin{array}{l}2 \\
2 \\
0\end{array}$ & $\begin{array}{r}143 \\
143 \\
0\end{array}$ & $\begin{array}{r}407 \\
407 \\
0\end{array}$ & $\begin{array}{r}2,933 \\
2,933 \\
0\end{array}$ & $\begin{array}{l}3 \\
3 \\
0\end{array}$ & $\begin{array}{r}313 \\
313 \\
0\end{array}$ & $\begin{array}{r}2,617 \\
2,617 \\
0\end{array}$ & $\begin{array}{r}16,309 \\
16,309 \\
0\end{array}$ \\
\hline Adjacont........ & $\begin{array}{l}\text { All } \\
\text { Offioial } \\
\text { Voluntary }\end{array}$ & $\begin{array}{r}132 \\
132 \\
0\end{array}$ & $\begin{array}{r}224 \\
224 \\
0\end{array}$ & $\begin{array}{r}10 \\
10 \\
0\end{array}$ & $\begin{array}{r}214 \\
214 \\
0\end{array}$ & $\begin{array}{l}0 \\
0 \\
0\end{array}$ & $\begin{array}{r}312 \\
312 \\
0\end{array}$ & $\begin{array}{r}15 \\
15 \\
0\end{array}$ & $\begin{array}{r}297 \\
297 \\
0\end{array}$ & $\begin{array}{l}0 \\
0 \\
0\end{array}$ & $\begin{array}{r}1,628 \\
1,628 \\
0\end{array}$ \\
\hline rural $1 \ldots \ldots \ldots$ & $\begin{array}{l}\text { All } \\
\text { Offioial } \\
\text { Voluntary } \\
\text { All } \\
\text { Offioial } \\
\text { Voluntary }\end{array}$ & $\begin{array}{r}1,574 \\
830 \\
744 \\
1,334 \\
1,334 \\
0\end{array}$ & $\begin{array}{r}2,262 \\
979 \\
1,283 \\
1,431 \\
1,431 \\
0\end{array}$ & $\begin{array}{l}0 \\
0 \\
0 \\
0 \\
0 \\
0\end{array}$ & $\begin{array}{r}2,137 \\
979 \\
1,209 \\
1,384 \\
1,384 \\
0\end{array}$ & $\begin{array}{r}75 \\
0 \\
75 \\
47 \\
47 \\
0\end{array}$ & $\begin{array}{r}2,719 \\
1,420 \\
1,299 \\
2,131 \\
2,131 \\
0\end{array}$ & $\begin{array}{r}0 \\
0 \\
0 \\
0 \\
0 \\
0\end{array}$ & $\begin{array}{r}2,628 \\
1,420 \\
1,208 \\
2,084 \\
2,084 \\
0\end{array}$ & $\begin{array}{r}90 \\
0 \\
90 \\
47 \\
47 \\
0\end{array}$ & $\begin{array}{r}18,373 \\
13,308 \\
5,065 \\
9,679 \\
9,579 \\
0\end{array}$ \\
\hline
\end{tabular}

1. For services other than examinations. 
Table 43a. Type of sorvioes in ohildren's dental olinies during one yoar, by oounty group 1n Kontuoky.

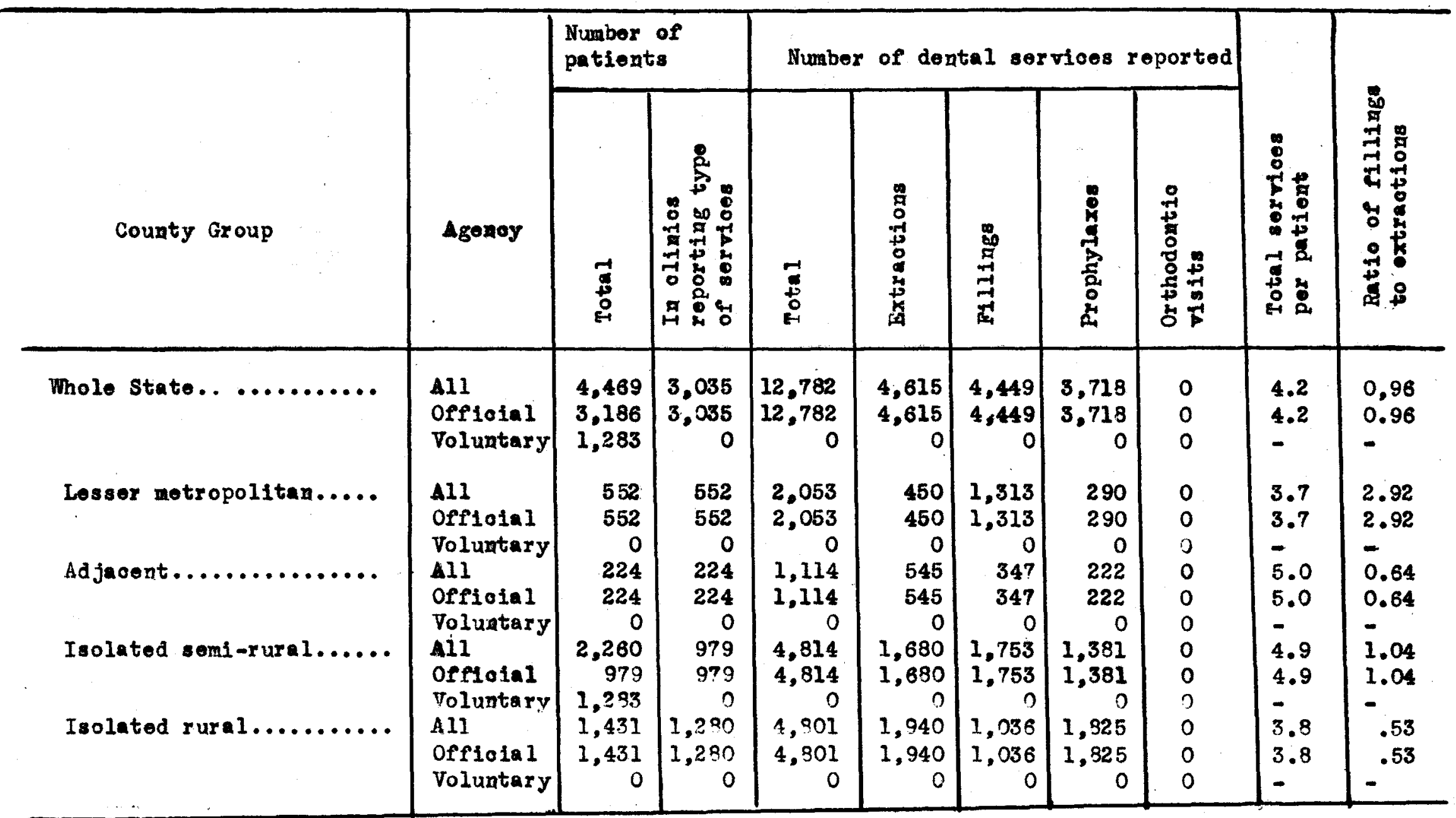


Table 44. Rates of oare in ohildren's dental olinios during one year, by oounty group in Kentuoky

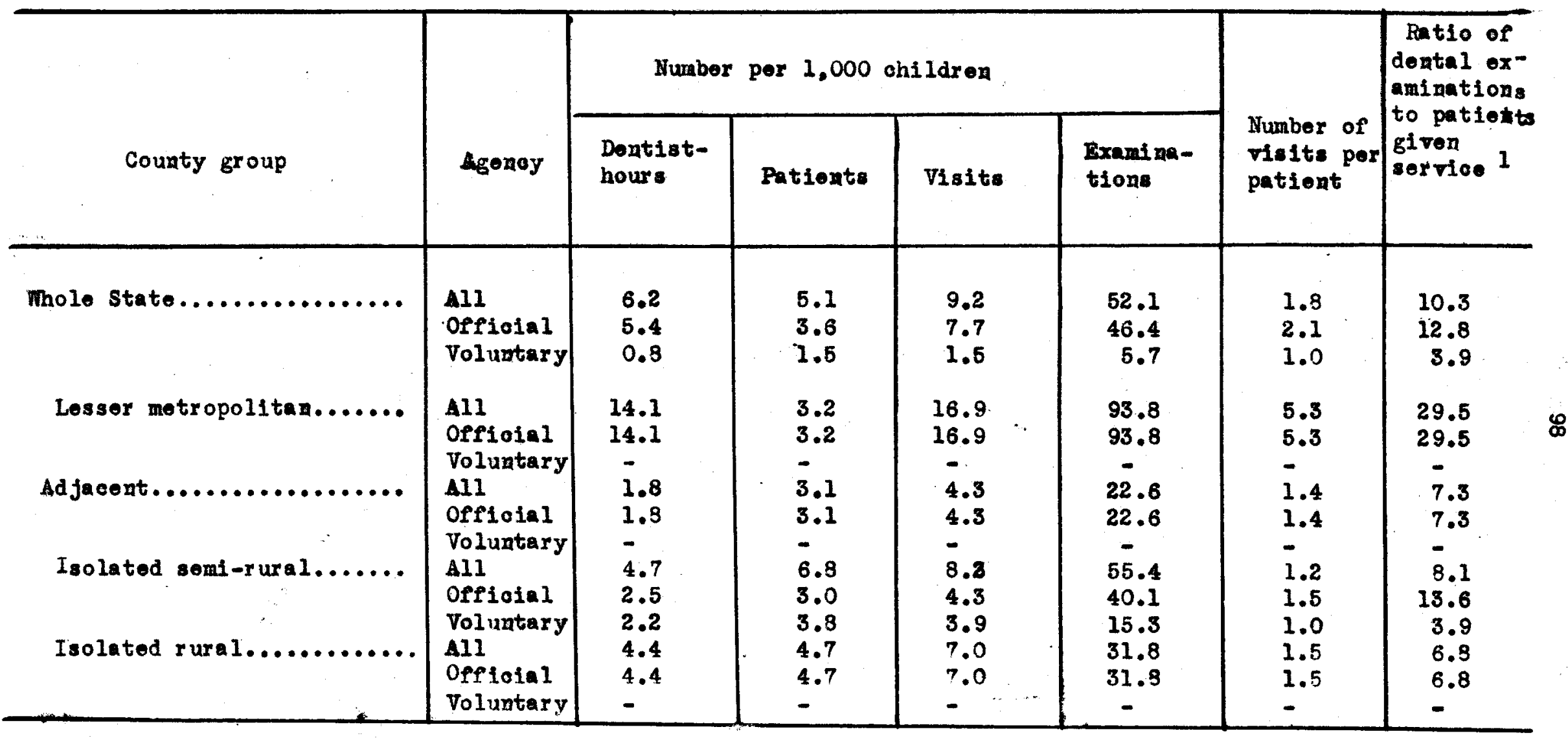

1. Table 43, 001. (10) divided by $001 .(2)$. 
1

Table 45. Mental hygiene servioes for ohildren during one year in Kentuoky.

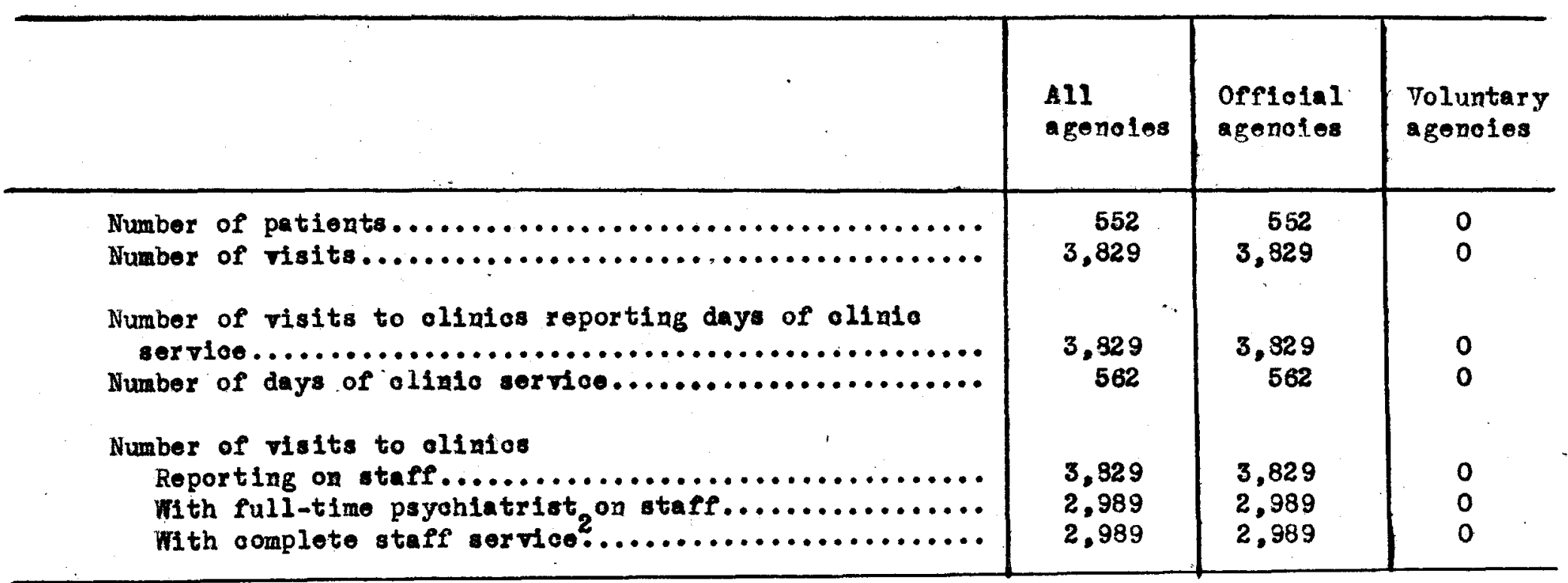

1. At olinios sorvod by psyohiatrists, psyohologists and/or podiatrioians.

2. Psychiatrist, psyohologist and sooial worker omployed full or part time.

Visits by county group

Motropolitan and adjeoent oounties.............. 2989

Isolated counties.......................... 840 
Table 46. Servioes for physloally handioappod ohildren during one year in Kentuoky.

\begin{tabular}{|c|c|c|c|}
\hline & All & $\begin{array}{l}\text { Offioial } \\
\text { agenoios }\end{array}$ & $\begin{array}{l}\text { Voluntary } \\
\text { agenoies }\end{array}$ \\
\hline 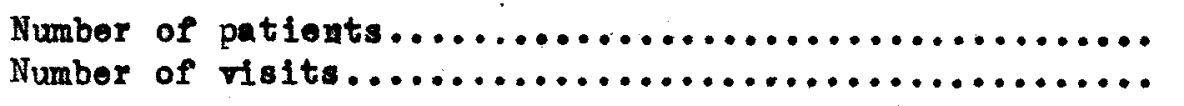 & $\begin{array}{r}5,051 \\
10,401\end{array}$ & $\begin{array}{l}4,525 \\
9,760\end{array}$ & $\begin{array}{l}526 \\
641\end{array}$ \\
\hline $\begin{array}{l}\text { Number of visits to ollnies reporting mmber of sessions } \\
\text { Number of sessions } \ldots \ldots \ldots \ldots \ldots \ldots \ldots \ldots \ldots \ldots \ldots \ldots \ldots \ldots \ldots \ldots\end{array}$ & $\begin{array}{l}10,401 \\
\quad 265\end{array}$ & $\begin{array}{r}9,760 \\
161\end{array}$ & $\begin{array}{l}641 \\
104\end{array}$ \\
\hline 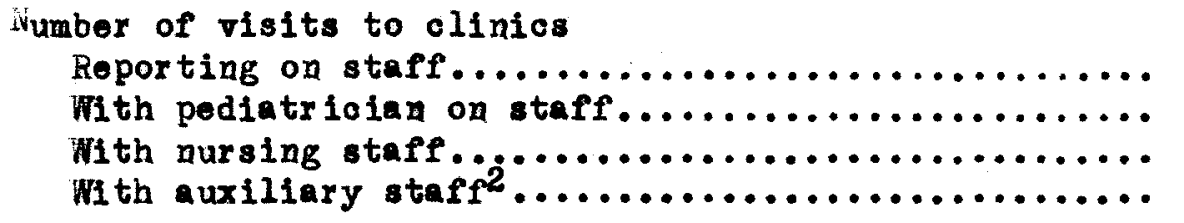 & $\begin{array}{r}10,401 \\
0 \\
10,156 \\
7,904\end{array}$ & $\begin{array}{r}9,760 \\
0 \\
9,515 \\
7,263\end{array}$ & $\begin{array}{r}641 \\
0 \\
641 \\
641\end{array}$ \\
\hline
\end{tabular}

1. At elinios sor red by medioal staff.

2. Physiotherapist, sooial worker or other professional atafe (exolusive of physioians and nurses) employed full or part time.

Visits by oounty group

Motropolitan and adjucent oounties............. 3949

Isolated countios......................... 6452 
Table 47. Children immunized by communtty health ageneles during one year, by oounty group in Kontuoky.

\begin{tabular}{|c|c|c|c|c|c|c|}
\hline \multirow{2}{*}{ County group } & \multicolumn{3}{|c|}{$\begin{array}{l}\text { Number of immunizations } \\
\text { reported } 1\end{array}$} & \multicolumn{3}{|c|}{$\begin{array}{l}\text { Inmunicationa per } \\
1000 \text { ohildren }\end{array}$} \\
\hline & $\operatorname{sman} 11$ pox & Diphtheria & $\begin{array}{l}\text { Whoopling } \\
\text { cough }\end{array}$ & $\operatorname{Sma1} 1 \mathrm{pox}$ & Diphtheria & $\begin{array}{l}\text { Whooping } \\
\text { cough }\end{array}$ \\
\hline $\begin{array}{l}\text { Wholo state............... } \\
\text { Greater motropolitan.... } \\
\text { Lessor metropolitan..... } \\
\text { Ad jacent.............. } \\
\text { Isolated semi-rural.... } \\
\text { Isolated rural......... }\end{array}$ & $\begin{array}{r}46,397 \\
9,413 \\
3,183 \\
19,786 \\
14,015\end{array}$ & $\begin{array}{r}40,848 \\
9,125 \\
1,995 \\
15,874 \\
13,954\end{array}$ & $\begin{array}{r}20,996 \\
2,522 \\
1,130 \\
8,265 \\
9,079\end{array}$ & $\begin{array}{l}52.6 \\
54.2 \\
44.1 \\
59.7 \\
46.0\end{array}$ & $\begin{array}{l}46.3 \\
52.5 \\
26.3 \\
47.9 \\
45.8\end{array}$ & $\begin{array}{l}23.8 \\
14.5 \\
15.7 \\
24.9 \\
29.8\end{array}$ \\
\hline
\end{tabular}

1. Number of immuaizations reported by agenoies in 97 counties. Not known whether immunizations are given in all other counties.

2. Adults are inoluded by 26 agenoies reporting a total of 13,489 imanizations. 
Table 48. Children 5-14 years of age in oouxties without sohool health servioes in publio elementary chools, by county group in Kentucky.

\begin{tabular}{|c|c|c|c|c|c|c|c|c|}
\hline \multirow[b]{2}{*}{ County group } & \multicolumn{3}{|c|}{ Number of counties } & \multicolumn{3}{|c|}{$\begin{array}{l}\text { Number of ohildren } \\
\text { in counties }\end{array}$} & \multicolumn{2}{|c|}{$\begin{array}{l}\text { Percent of child- } \\
\text { ren in counties }\end{array}$} \\
\hline & Total & $\begin{array}{l}\text { Without } \\
\text { medios } 1 \\
\text { servioe } 1\end{array}$ & $\begin{array}{l}\text { Without } \\
\text { modical or } \\
\text { nursing } \\
\text { sorvioe }\end{array}$ & Total & $\begin{array}{l}\text { Without } \\
\text { medionl } \\
\text { servioe }\end{array}$ & $\begin{array}{l}\text { mithout } \\
\text { medionl or } \\
\text { nursing } \\
\text { service }\end{array}$ & $\begin{array}{l}\text { Without } \\
\text { modical } \\
\text { service }\end{array}$ & $\begin{array}{l}\text { Without } \\
\text { medionl or } \\
\text { nursing } \\
\text { service }\end{array}$ \\
\hline $\begin{array}{l}\text { Whole state....... } \\
\text { Greater metro- } \\
\text { politan........ } \\
\text { Lesser metropol- } \\
\text { Itan............ } \\
\text { Adjaoent........ } \\
\text { Isolated semi- } \\
\text { rural......... } \\
\text { Isolated rural... }\end{array}$ & $\begin{array}{r}5 \\
15 \\
\\
37 \\
63\end{array}$ & $\begin{array}{r}1 \\
4 \\
11 \\
28\end{array}$ & $\begin{array}{r}0 \\
4 \\
4 \\
4 \\
19\end{array}$ & $\begin{array}{r}106,755 \\
44,634 \\
200,282 \\
183,644\end{array}$ & $\begin{array}{r}8,978 \\
9,275 \\
61,983 \\
92,168\end{array}$ & $\begin{array}{r}0 \\
9,275 \\
19,499 \\
58,169\end{array}$ & $\begin{array}{r}8.4 \\
20.8 \\
\\
30.9 \\
44.7\end{array}$ & $\begin{array}{r}- \\
20.8 \\
9.7 \\
31.7\end{array}$ \\
\hline
\end{tabular}

Note: Exoludes Federal achools for Indians.

1. A county is said to be without school modical service if there is not at least one public elementary sohool in which medical examinations by a physician are done on (1) all pupils once a year, (2) oortain grades once year, or (3) referrals by teachers or aurses.

2. Table 61, 001 (1) minus 001. (2). 
Table 49. Staff engeged in sohool hoalth services, by oounty group in Kentucky.

\begin{tabular}{|c|c|c|c|c|c|c|c|c|c|c|c|c|c|c|c|}
\hline \multirow[b]{4}{*}{ County group } & \multicolumn{9}{|c|}{ MEDICAL STAFF } & \multicolumn{6}{|c|}{ NURSTNG STAEF } \\
\hline & \multirow{3}{*}{ 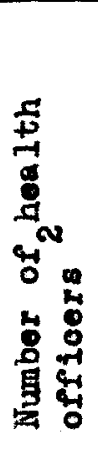 } & \multicolumn{8}{|c|}{ Number of sohool physioians } & \multirow{3}{*}{ 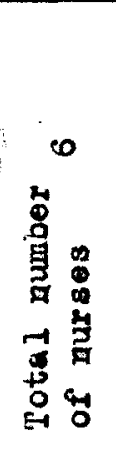 } & \multirow{2}{*}{\multicolumn{4}{|c|}{ Number employed by - }} & \multirow{3}{*}{ 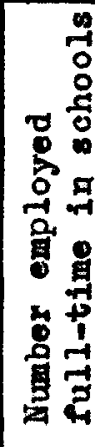 } \\
\hline & & & \multicolumn{3}{|c|}{ Specialty } & \multicolumn{4}{|c|}{ Fmployed by - } & & & & & & \\
\hline & & 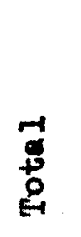 & 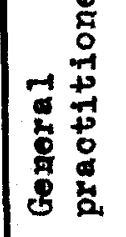 & 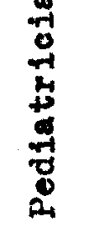 & 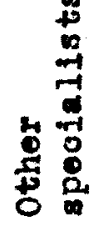 & 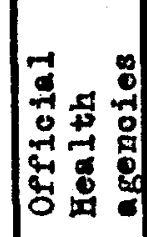 & 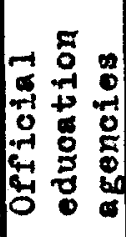 & 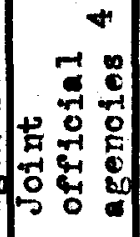 & 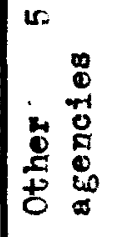 & & 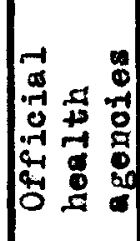 & 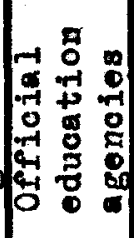 & 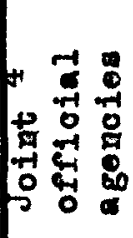 & 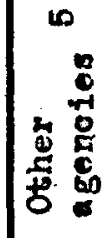 & \\
\hline $\begin{array}{l}\text { Whole stato.......... } \\
\text { Lesser metropolitan.. } \\
\text { Adjacent............ } \\
\text { Isolated semi-rural.. } \\
\text { Isolated rural....... }\end{array}$ & $\begin{array}{r}74 \\
3 \\
10 \\
27 \\
34\end{array}$ & $\begin{array}{r}14 \\
6 \\
3 \\
3 \\
2\end{array}$ & $\begin{array}{r}13 \\
6 \\
3 \\
2 \\
2\end{array}$ & $\begin{array}{l}1 \\
0 \\
0 \\
1 \\
0\end{array}$ & $\begin{array}{l}0 \\
0 \\
0 \\
0 \\
0\end{array}$ & $\begin{array}{r}10 \\
6 \\
0 \\
2 \\
2\end{array}$ & $\begin{array}{l}0 \\
0 \\
0 \\
0 \\
0\end{array}$ & $\begin{array}{l}1 \\
0 \\
0 \\
1 \\
0\end{array}$ & $\begin{array}{l}3 \\
0 \\
3 \\
0 \\
0\end{array}$ & $\begin{array}{r}194 \\
54 \\
17 \\
77 \\
46\end{array}$ & $\begin{array}{r}167 \\
54 \\
11 \\
64 \\
38\end{array}$ & $\begin{array}{l}1 \\
0 \\
0 \\
1 \\
0\end{array}$ & $\begin{array}{r}23 \\
0 \\
5 \\
10 \\
8\end{array}$ & $\begin{array}{l}3 \\
0 \\
1 \\
2 \\
0\end{array}$ & $\begin{array}{l}2 \\
0 \\
1 \\
1 \\
0\end{array}$ \\
\hline
\end{tabular}

1. Wedical staff inoluded only whon modical examinations are done (1) on all pupils onoe a year, (2) certain grades once year, or (3) referrals by teachers or nurses.

2. A health officer may be counted more than onoe if he 80 rves more than one agenoy.

3. A school physician may be counted more than once if he werves more than one agonoy. Inoludes physicians working full or part-time in schools.

4. Joint offloial health and eduoation agenoies operating a joint or cooperativo program.

5. Other agenoies inolude roluntary agenofes and offiolal agenoies other than health and eduoation.

6. If a nurse serves more than one county, oonsidered only in the county of her headquarters. This is an unduplioated count of nurses working full or part-time in sohools. 
Table 50. Services for children provided by official and roluntary publie health nursing agencies during one year, by oounty group in Kentuoky.

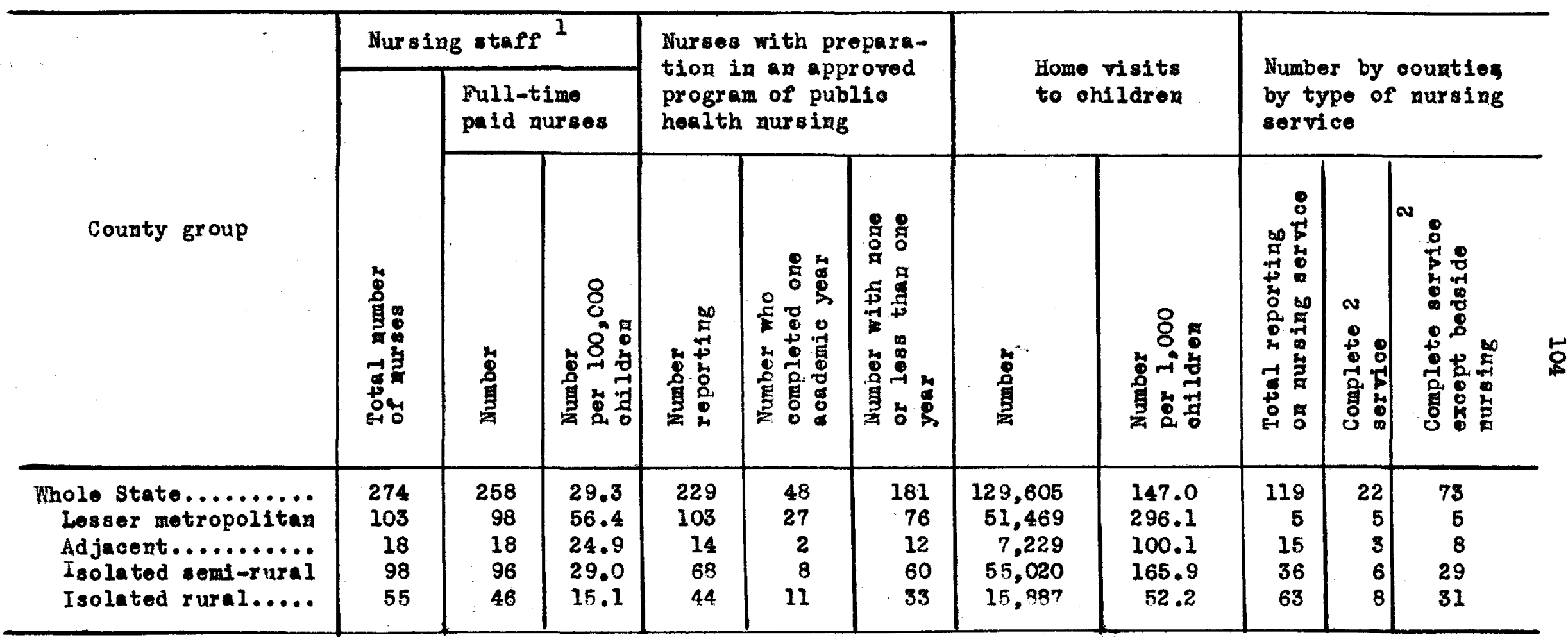

1. Exclusive of nurses employed by agenoies giving only school health, industrial, tuberculosis, or venereal disease services; exolusive of nurses reported as supervisors employed by state agenoies. If a nurse serve more than one county, considered inly in the county of her headquarters. This is an unduplioated oount.

2. Assistance in well-ohild olinics, home visits for health supervision, bedside nursing oare and sohool services. 
Table 51. Child population, estimated as of July 1, 1945, by oounty group and individual county in Kontuoky.

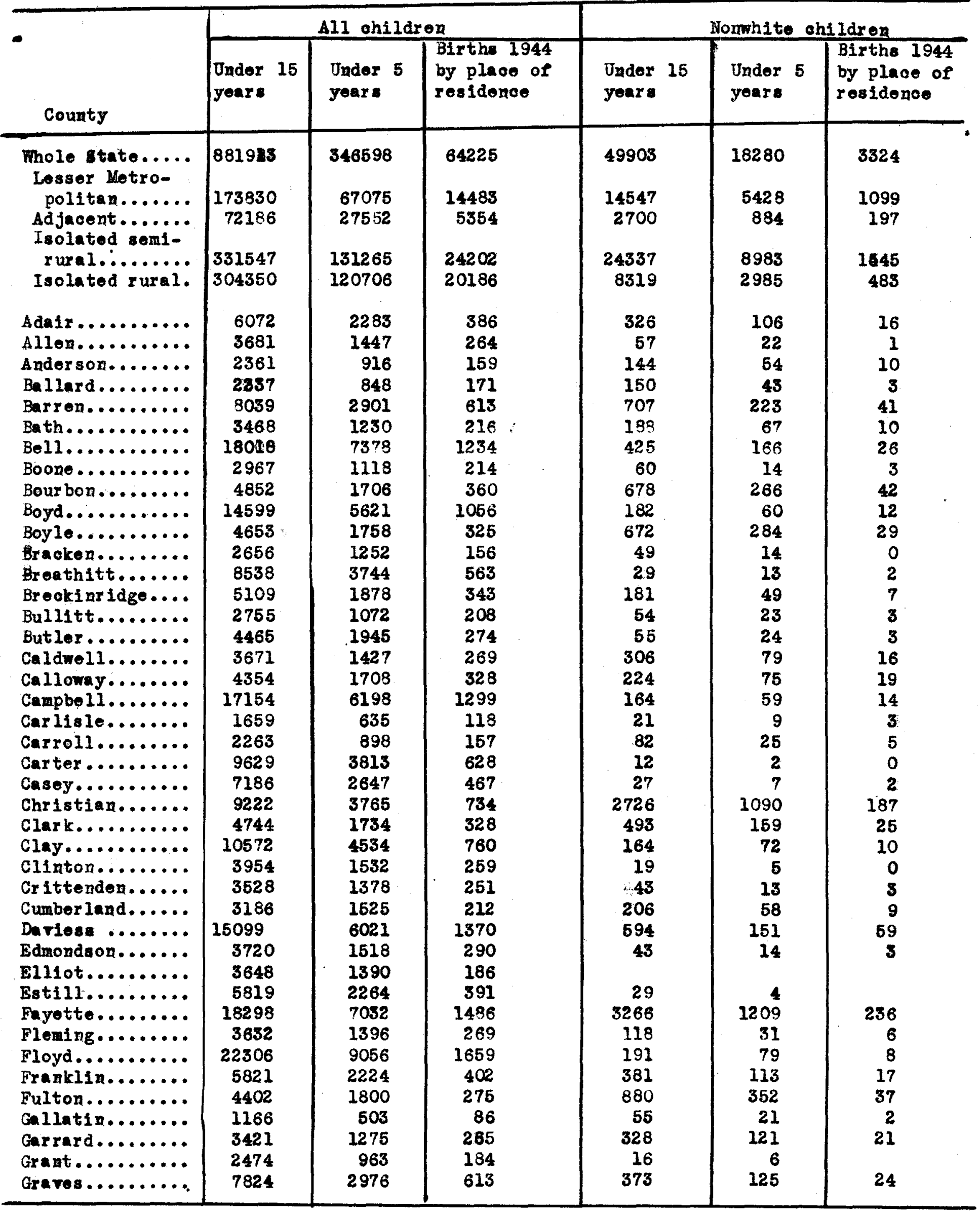


Table 51. (cont'd). Child population, ostinated as of July 1, 1945, by oounty group and $i$ solviduni county in Kentueky.

\begin{tabular}{|c|c|c|c|c|c|c|}
\hline \multirow[b]{2}{*}{ County } & \multicolumn{3}{|c|}{ A11 ohildren } & \multicolumn{3}{|c|}{ Norwhite ohildren } \\
\hline & $\begin{array}{l}\text { Undor } 16 \\
\text { years }\end{array}$ & $\begin{array}{l}\text { Under } 5 \\
\text { years }\end{array}$ & $\begin{array}{l}\text { Birthe } 1944 \\
\text { by plaoe of } \\
\text { residenoe }\end{array}$ & $\begin{array}{l}\text { Under } 15 \\
\text { years }\end{array}$ & $\begin{array}{l}\text { Jader } 5 \\
\text { yoar: }\end{array}$ & $\begin{array}{l}\text { Birthe 1944 } \\
\text { by place of } \\
\text { residenoe }\end{array}$ \\
\hline 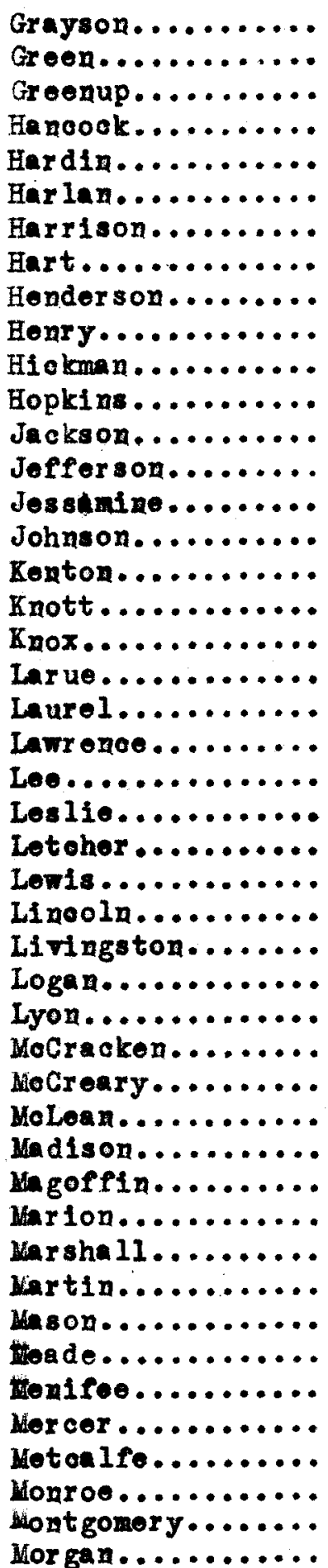 & $\begin{array}{r}5161 \\
3623 \\
8315 \\
1739 \\
8063 \\
29296 \\
3497 \\
4719 \\
8015 \\
3213 \\
2329 \\
9963 \\
6150 \\
110821 \\
3079 \\
9217 \\
23241 \\
9037 \\
12011 \\
2952 \\
9107 \\
5231 \\
3500 \\
6396 \\
16570 \\
5312 \\
6741 \\
2525 \\
6436 \\
1940 \\
11624 \\
7045 \\
3415 \\
8438 \\
6865 \\
5876 \\
3921 \\
4527 \\
4872 \\
2549 \\
1960 \\
3682 \\
3219 \\
4633 \\
3591 \\
6062\end{array}$ & $\begin{array}{r}2112 \\
1227 \\
3138 \\
736 \\
3508 \\
12228 \\
1427 \\
1937 \\
3118 \\
1173 \\
895 \\
3815 \\
2260 \\
43445 \\
1171 \\
3749 \\
8693 \\
3703 \\
4450 \\
1132 \\
3447 \\
1982 \\
1240 \\
2488 \\
6530 \\
2062 \\
2577 \\
994 \\
2550 \\
731 \\
4491 \\
2783 \\
1426 \\
3220 \\
2964 \\
2317 \\
1619 \\
1850 \\
1905 \\
1066 \\
732 \\
1312 \\
1202 \\
1848 \\
1517 \\
2378\end{array}$ & $\begin{array}{r}360 \\
199 \\
567 \\
118 \\
579 \\
2139 \\
241 \\
325 \\
683 \\
226 \\
145 \\
777 \\
414 \\
9666 \\
192 \\
580 \\
1779 \\
620 \\
827 \\
190 \\
651 \\
332 \\
165 \\
428 \\
1256 \\
350 \\
432 \\
166 \\
441 \\
116 \\
938 \\
502 \\
241 \\
603 \\
364 \\
462 \\
287 \\
348 \\
395 \\
193 \\
124 \\
258 \\
197 \\
366 \\
242 \\
345\end{array}$ & $\begin{array}{r}23 \\
284 \\
42 \\
53 \\
310 \\
1956 \\
176 \\
522 \\
1102 \\
270 \\
339 \\
803 \\
4 \\
12368 \\
339 \\
5 \\
731 \\
91 \\
136 \\
188 \\
107 \\
12 \\
25 \\
8 \\
494 \\
1 \\
318 \\
16 \\
829 \\
104 \\
1343 \\
16 \\
48 \\
699 \\
14 \\
400 \\
29 \\
1 \\
553 \\
97 \\
9 \\
259 \\
166 \\
90 \\
441 \\
1\end{array}$ & $\begin{array}{r}6 \\
69 \\
16 \\
21 \\
101 \\
739 \\
59 \\
130 \\
399 \\
87 \\
130 \\
334 \\
0 \\
4616 \\
125 \\
2 \\
295 \\
20 \\
51 \\
68 \\
33 \\
4 \\
10 \\
1 \\
187 \\
0 \\
116 \\
5 \\
312 \\
18 \\
527 \\
6 \\
22 \\
199 \\
7 \\
119 \\
11 \\
1 \\
183 \\
38 \\
5 \\
86 \\
59 \\
23 \\
268 \\
1\end{array}$ & $\begin{array}{r}1 \\
15 \\
4 \\
6 \\
18 \\
125 \\
8 \\
36 \\
80 \\
16 \\
20 \\
67 \\
0 \\
931 \\
22 \\
0 \\
62 \\
3 \\
8 \\
12 \\
7 \\
0 \\
1 \\
1 \\
41 \\
0 \\
21 \\
1 \\
50 \\
3 \\
105 \\
2 \\
4 \\
33 \\
0 \\
30 \\
1 \\
0 \\
35 \\
8 \\
0 \\
15 \\
7 \\
5 \\
18 \\
0\end{array}$ \\
\hline
\end{tabular}


Table 51 (cont'd). Child population, ostimated as of July 1, 1945, by eounty group and indiridual county in Kentucky.

\begin{tabular}{|c|c|c|c|c|c|c|}
\hline \multirow[b]{2}{*}{ County } & \multicolumn{3}{|c|}{ All ohildren } & \multicolumn{3}{|c|}{ Nomwhite ohildren } \\
\hline & $\begin{array}{l}\text { Ondor } 15 \\
\text { yoars }\end{array}$ & $\begin{array}{l}\text { Undor } 5 \\
\text { years }\end{array}$ & $\begin{array}{l}\text { Births } 1844 \\
\text { by place of } \\
\text { residenoe }\end{array}$ & $\begin{array}{l}\text { Jnder } 15 \\
\text { yoars }\end{array}$ & $\begin{array}{l}\text { Under } 5 \\
\text { yoars }\end{array}$ & $\begin{array}{l}\text { Births } 1944 \\
\text { by place of } \\
\text { residenoe }\end{array}$ \\
\hline 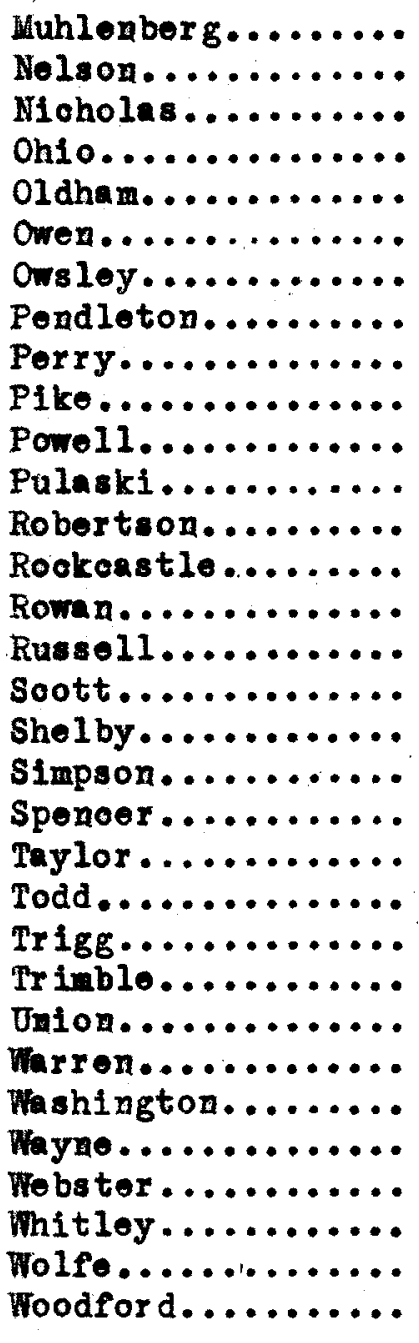 & $\begin{array}{r}11644 \\
5785 \\
2031 \\
6383 \\
2141 \\
2417 \\
3853 \\
2358 \\
20968 \\
31666 \\
2600 \\
13296 \\
894 \\
6561 \\
4692 \\
4574 \\
3379 \\
4662 \\
2982 \\
1978 \\
4337 \\
3734 \\
3591 \\
1566 \\
4872 \\
9795 \\
4337 \\
6887 \\
4724 \\
11923 \\
3712 \\
3142\end{array}$ & $\begin{array}{r}4914 \\
2481 \\
718 \\
2744 \\
675 \\
926 \\
165 \\
800 \\
8917 \\
12680 \\
969 \\
4910 \\
364 \\
2680 \\
1938 \\
1655 \\
1263 \\
1662 \\
1128 \\
823 \\
1589 \\
1400 \\
1664 \\
537 \\
1833 \\
3718 \\
1759 \\
2953 \\
1699 \\
4718 \\
1461 \\
1034\end{array}$ & $\begin{array}{r}894 \\
467 \\
140 \\
432 \\
156 \\
132 \\
157 \\
123 \\
1440 \\
2211 \\
171 \\
897 \\
66 \\
415 \\
353 \\
192 \\
266 \\
842 \\
204 \\
154 \\
281 \\
272 \\
213 \\
98 \\
396 \\
794 \\
290 \\
440 \\
341 \\
781 \\
240 \\
211\end{array}$ & $\begin{array}{r}518 \\
407 \\
112 \\
133 \\
198 \\
90 \\
34 \\
29 \\
677 \\
268 \\
63 \\
190 \\
17 \\
8 \\
9 \\
67 \\
404 \\
585 \\
346 \\
136 \\
344 \\
822 \\
706 \\
1 \\
497 \\
1168 \\
463 \\
139 \\
416 \\
69 \\
1 \\
396\end{array}$ & $\begin{array}{r}185 \\
143 \\
37 \\
43 \\
43 \\
31 \\
9 \\
7 \\
258 \\
91 \\
16 \\
51 \\
5 \\
4 \\
6 \\
22 \\
139 \\
203 \\
117 \\
39 \\
96 \\
347 \\
337 \\
0 \\
182 \\
380 \\
174 \\
68 \\
172 \\
26 \\
0 \\
135\end{array}$ & $\begin{array}{r}31 \\
33 \\
9 \\
6 \\
7 \\
5 \\
1 \\
2 \\
37 \\
20 \\
3 \\
13 \\
2 \\
0 \\
0 \\
3 \\
36 \\
48 \\
20 \\
7 \\
21 \\
54 \\
33 \\
0 \\
39 \\
63 \\
42 \\
11 \\
21 \\
3 \\
0 \\
18\end{array}$ \\
\hline
\end{tabular}


Tablo 66. Report year for ohild health servioes in Kentuoky.

\begin{tabular}{|c|c|c|c|c|c|c|c|c|c|c|}
\hline \multirow[b]{3}{*}{ Year ended ${ }^{1}$} & \multicolumn{10}{|c|}{ Number of sohodulos } \\
\hline & \multicolumn{2}{|c|}{ Hospitals } & \multirow[b]{2}{*}{ 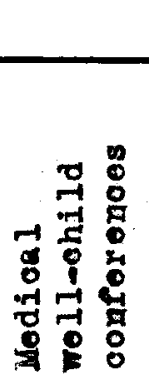 } & $\ddots$ & \multicolumn{5}{|c|}{ Conmunity health servioes } & \\
\hline & $\begin{array}{l}\vec{a} \\
\overrightarrow{0} \\
\overrightarrow{0}\end{array}$ & 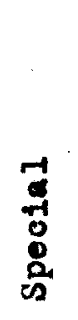 & & 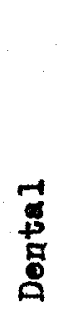 & 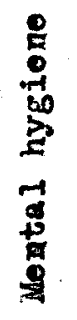 & 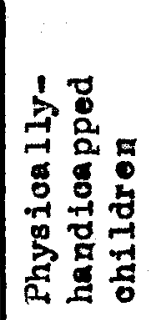 & 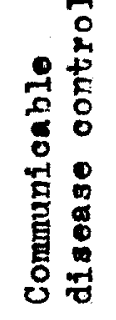 & 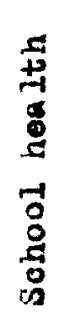 & 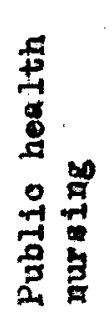 & \\
\hline Whole state......... & 97 & 6 & 53 & 21 & 2 & 31 & 97 & 94 & 106 & . \\
\hline $\begin{array}{l}\text { December } 31,1344 \ldots \\
\text { June } 30,1945 \ldots \ldots \\
\text { December } 31,1945 \ldots \\
\text { June } 30,1946 \ldots \ldots \\
\text { Deoember } 31,1946 \ldots \\
\text { June } 30,1947 \ldots \ldots \ldots \\
\text { Not repor ted...... }\end{array}$ & $\begin{array}{r}1 \\
7 \\
82 \\
2 \\
3 \\
0 \\
2\end{array}$ & $\begin{array}{l}0 \\
1 \\
4 \\
1 \\
0 \\
0 \\
0\end{array}$ & $\begin{array}{r}0 \\
0 \\
49 \\
0 \\
2 \\
0 \\
2\end{array}$ & $\begin{array}{r}0 \\
0 \\
20 \\
0 \\
0 \\
0 \\
1\end{array}$ & $\begin{array}{l}0 \\
0 \\
2 \\
0 \\
0 \\
0 \\
0\end{array}$ & $\begin{array}{r}0 \\
0 \\
31 \\
0 \\
0 \\
0 \\
0\end{array}$ & $\begin{array}{r}0 \\
0 \\
97 \\
0 \\
0 \\
0 \\
0\end{array}$ & $\begin{array}{r}0 \\
0 \\
88 \\
0 \\
1 \\
0 \\
5\end{array}$ & $\begin{array}{r}0 \\
0 \\
103 \\
0 \\
0 \\
0 \\
3\end{array}$ & \\
\hline
\end{tabular}

Note 1: Date of first mailing of sohedules:

Physiolans - Soptember, 1948

Dentists - Septenber, 1946

2

Note 2, Pediatrioians' rooords were for the following months : Total - 31

$\begin{array}{llll}\frac{1946}{\text { June }-19} & \text { Soptomber } & -3 & \text { Decomber }-1 \\ \text { July }-3 & \text { Ooteber } & -1 & \\ \text { August }-3 & \text { Notember } & -1\end{array}$

1. Ootober 1 through Maroh 31 considered as Deoember 31; April 1 through September 30 oonsidered as June 30.

2. Four weeks onded between January 15 and February 14 oonsidered as January, oto. 
Table 67. Alphabetioal list of Kontuoky countios acoording to population and proximity to densely populated areas.

Koy: $\begin{aligned} \text { H } & \text { - lesser motropolitan } \\ \text { IS } & - \text { is jacont } \\ \text { IR } & \text { - isolated semi-rural }\end{aligned}$

COUNTY

COUNTY GROUP

COUNTY

COUNTY GROUP

Adair

IR

Allon IR

Anders on. IR

Ballard IR

Barron IS

Bath IR

Boll IS

Boone A

Bourbon IS

Boyd IM

Boyle Is

Bracken IR

Breathitt IR

Brookinridgo IR

Bullitt

Butlor IR

Caldwoll Is

Calloway is

Campbell LM

Carlisle IR

Carroll. IS

Carter A

Casey IR

Christian IS

Clark IS

Clay IR

Clinton IR

Crittenden

Cumberland IR

Daviess A

Edmonson IR

Elliot IR

Est111 IS

Fayette IS

Fleming IR

Floyd IR

Franklin Is

Fulton IS

Gallatin IR

Garrard

IR

$\begin{array}{lr}\text { Grant } & \text { A } \\ \text { Graves } & \text { IS } \\ \text { Grayson } & \text { IR } \\ \text { Groon } & \text { IR } \\ \text { Groonup } & \text { A }\end{array}$

Hanoook IR

Hardin IS

Hiarlax IS

Harrison IS

Hart IR

Henderson LM

HonYy IR

Hioloma IR

Hopkins IS

Jaoks on IR

Jofferson LM

Jossamine IS

Johnson IR

Kenton IM

Inott IR

Knox IR

Laruo IR

Laure1 IR

Lantenoo : A

Leo IR

Los lis IR

Letoher IS

Lowis IR

Linooln IR

Livingston IR

Logax IS

Lyon . IR

MoCraoken IS

MoCreary IR

MoLena A

Madison Is

Yagorfin IR

Marion IS

Harsha11 IR

Naxtia IR 
Table 67 (oont'd). Alphabotioal list of Kentucky countios aocording to population and proxinity to donsely populated areas.

\begin{tabular}{|c|c|c|c|}
\hline COUNTY & COTNTY GROTP & COUNTY & COUNTY GROUP \\
\hline $\begin{array}{l}\text { Mason } \\
\text { Meade } \\
\text { Henifoe } \\
\text { Mercer } \\
\text { Hetealfe }\end{array}$ & $\begin{array}{l}\text { IS } \\
\text { IR } \\
\text { IR } \\
\text { IS } \\
\text { IR }\end{array}$ & $\begin{array}{l}\text { Robertson } \\
\text { Rookoastlo } \\
\text { Kowan } \\
\text { Russell } \\
\text { Soott }\end{array}$ & $\begin{array}{l}\text { IR } \\
\text { IR } \\
\text { IR } \\
\text { IR } \\
\text { IS }\end{array}$ \\
\hline $\begin{array}{l}\text { Monroe } \\
\text { Montgomery } \\
\text { Morgan } \\
\text { Muhlenber } \mathrm{B} \\
\text { Nelson }\end{array}$ & $\begin{array}{l}\text { IR } \\
\text { IS } \\
\text { IR } \\
\text { IS } \\
\text { IS }\end{array}$ & $\begin{array}{l}\text { Sholby } \\
\text { Simpa on } \\
\text { Sponees } \\
\text { Taylor } \\
\text { Todd }\end{array}$ & $\begin{array}{r}\mathbf{A} \\
\mathrm{IS} \\
\mathbf{4} \\
\mathrm{IR} \\
\mathrm{IR}\end{array}$ \\
\hline $\begin{array}{l}\text { Nioholas } \\
\text { Ohio } \\
\text { Oldham } \\
\text { Owon } \\
\text { Orsloy }\end{array}$ & $\begin{array}{r}\text { IR } \\
\text { IR } \\
\text { IR } \\
\text { IR }\end{array}$ & $\begin{array}{l}\text { Trigg } \\
\text { Trimble } \\
\text { Union } \\
\text { hirron } \\
\text { Timehington }\end{array}$ & $\begin{array}{r}\text { IR } \\
4 \\
\text { IS } \\
\text { IR }\end{array}$ \\
\hline $\begin{array}{l}\text { Pendleton } \\
\text { Perry } \\
\text { Pike } \\
\text { Powell } \\
\text { Pulaski }\end{array}$ & $\begin{array}{l}\text { A } \\
\text { IS } \\
\text { IS } \\
\text { IR } \\
\text { IS }\end{array}$ & $\begin{array}{l}\text { Thymo } \\
\text { Wobster } \\
\text { Whitloy } \\
\text { Wolfo } \\
\text { Woodford }\end{array}$ & $\begin{array}{l}\text { IR } \\
\text { IS } \\
\text { IR } \\
\text { IS }\end{array}$ \\
\hline
\end{tabular}


AFPENDIX B 
Tablo 1. Porcent of ohildren under 15: Dnited States and oa oh state, 1945.

\begin{tabular}{|c|c|c|c|c|}
\hline State & $\begin{array}{l}1 \\
\text { Total } \\
\text { oivilian } \\
\text { population } \\
\text { (Thousands) }\end{array}$ & $\begin{array}{l}\text { Number } \\
\text { ohtldren } \\
\text { under } \\
\text { age } 15 \\
\text { (Thousands) }\end{array}$ & $\begin{array}{l}\text { Peroent } \\
\text { ohildren } \\
\text { under } \\
\text { age } 15\end{array}$ & Rank \\
\hline 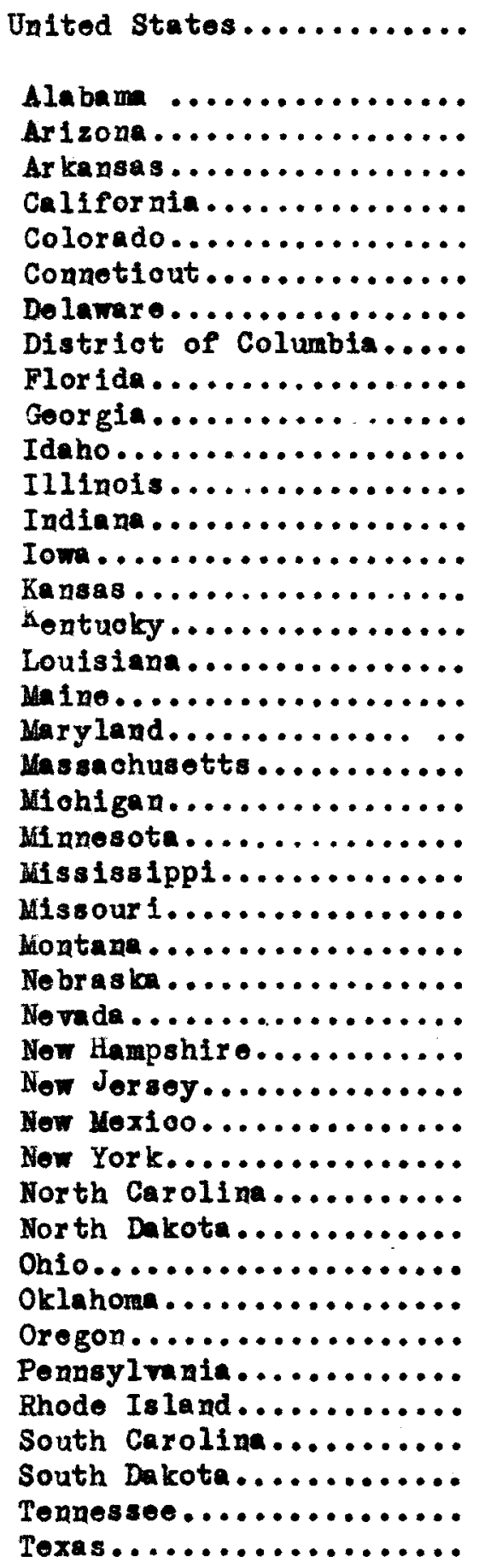 & $\begin{array}{r}127,452 \\
2,626 \\
540 \\
1,788 \\
8,391 \\
1,015 \\
1,776 \\
266 \\
824 \\
1,984 \\
2,851 \\
449 \\
7,312 \\
3,466 \\
2,368 \\
1,700 \\
2,540 \\
2,286 \\
797 \\
1,906 \\
4,087 \\
5,552 \\
2,613 \\
2,035 \\
3,479 \\
447 \\
1,186 \\
118 \\
461 \\
3,814 \\
489 \\
12,574 \\
3,342 \\
516 \\
6,808 \\
2,030 \\
1,303 \\
9,248 \\
673 \\
1,804 \\
515 \\
2,776 \\
6,244\end{array}$ & $\begin{array}{r}36,006 \\
993 \\
184 \\
584 \\
1,384 \\
307 \\
412 \\
70 \\
149 \\
559 \\
1,036 \\
157 \\
1,825 \\
919 \\
647 \\
464 \\
882 \\
776 \\
236 \\
504 \\
965 \\
1,494 \\
710 \\
763 \\
964 \\
146 \\
321 \\
34 \\
117 \\
925 \\
200 \\
2,831 \\
1,212 \\
181 \\
1,731 \\
692 \\
326 \\
2,372 \\
167 \\
713 \\
172 \\
958 \\
2,262\end{array}$ & $\begin{array}{l}28.3 \\
37.8 \\
34.1 \\
32.7 \\
22.5 \\
30.3 \\
23.2 \\
26.3 \\
19.0 \\
28.2 \\
36.3 \\
34.9 \\
25.0 \\
26.5 \\
27.3 \\
27.3 \\
34.7 \\
33.9 \\
29.6 \\
26.4 \\
23.6 \\
26.9 \\
27.1 \\
37.5 \\
27.7 \\
32.6 \\
27.1 \\
28.5 \\
25.5 \\
24.2 \\
40.9 \\
22.5 \\
36.3 \\
35.1 \\
25.4 \\
34.1 \\
25.0 \\
25.6 \\
24.8 \\
39.5 \\
33.3 \\
34.5 \\
36.2\end{array}$ & $\begin{array}{r}- \\
3 \\
14 \\
19 \\
47 \\
21 \\
46 \\
36 \\
49 \\
25 \\
5 \\
11 \\
40 \\
34 \\
28 \\
28 \\
12 \\
16 \\
23 \\
35 \\
45 \\
33 \\
31 \\
4 \\
27 \\
20 \\
31 \\
24 \\
38 \\
44 \\
1 \\
47 \\
5 \\
10 \\
39 \\
14 \\
40 \\
37 \\
43 \\
2 \\
17 \\
13 \\
7\end{array}$ \\
\hline
\end{tabular}


Table 1 (oont'd). Poroont of ohildron under 15: United Statos and each state, 1945.

\begin{tabular}{|c|c|c|c|c|}
\hline Stato & $\begin{array}{l}\text { Total } \\
\text { ofvilian } \\
\text { population } \\
\text { (Thousands) }\end{array}$ & $\begin{array}{l}\text { Number } \\
\text { ohildren } \\
\text { under } \\
\text { ge } 15 \\
\text { (Thousands) }\end{array}$ & $\begin{array}{l}\text { Pereont } \\
\text { ohildren } \\
\text { under } \\
\text { ago } 15\end{array}$ & Rank \\
\hline 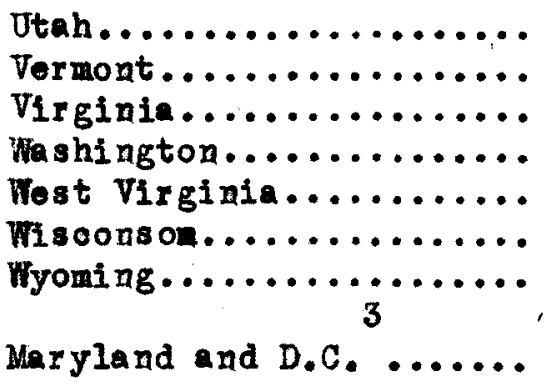 & $\begin{array}{r}575 \\
328 \\
2,665 \\
2,027 \\
1,687 \\
2,930 \\
236 \\
2,730\end{array}$ & $\begin{array}{r}203 \\
93 \\
880 \\
508 \\
611 \\
796 \\
71 \\
653\end{array}$ & $\begin{array}{l}35.3 \\
28.2 \\
33.0 \\
25.0 \\
36.2 \\
27.2 \\
30.1 \\
23.9\end{array}$ & $\begin{array}{r}9 \\
25 \\
18 \\
40 \\
7 \\
30 \\
22 \\
-\end{array}$ \\
\hline
\end{tabular}

1. Current population roports: Population estimates, S.P. 25, No.2, Aug. 15, 1947. Bureau of Consus, U.S. Dopt. of Comeros.

2. Estimatos by the Amorioan Aoadomy of Podiatrics Study of Child Health Servioes.

3. Excludos Arlington and Fairfax counties in Virginia. 
Table 2. Ago diusted death rates per 1,000 population 1940: Tnited States and each state. (By place of oocurence).

\begin{tabular}{|c|c|c|}
\hline State & $\begin{array}{l}\text { Age adjusted } \\
\text { death rate }\end{array}$ & Rank \\
\hline 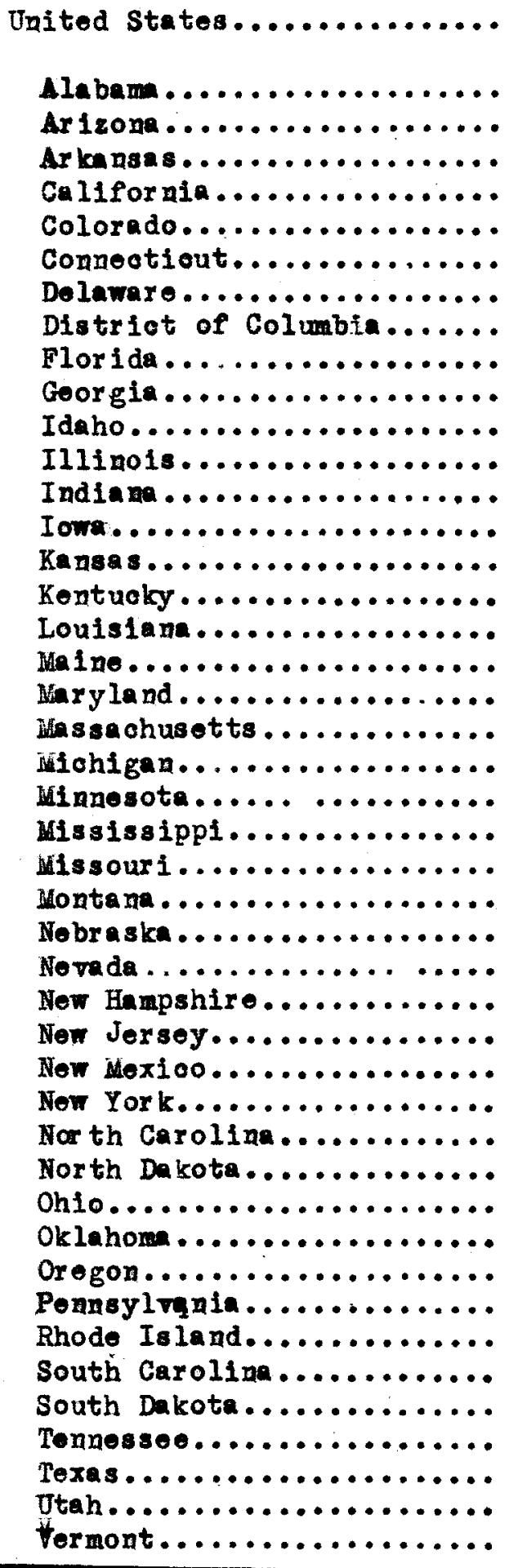 & $\begin{array}{r}10.7 \\
12.5 \\
12.9 \\
9.9 \\
10.3 \\
10.3 \\
9.8 \\
11.3 \\
13.4 \\
12.3 \\
12.3 \\
.9 .8 \\
10.6 \\
10.4 \\
8.6 \\
8.7 \\
10.8 \\
12.7 \\
10.2 \\
12.3 \\
10.3 \\
10.2 \\
8.8 \\
12.3 \\
10.1 \\
10.2 \\
8.5 \\
12.9 \\
10.0 \\
10.8 \\
11.7 \\
10.9 \\
11.4 \\
8.6 \\
10.4 \\
9.4 \\
9.6 \\
11.4 \\
10.5 \\
13.5 \\
8.2 \\
11.4 \\
10.9 \\
10.0 \\
10.3\end{array}$ & $\begin{array}{r}- \\
44 \\
46 \\
13 \\
21 \\
21 \\
10 \\
34 \\
48 \\
39 \\
39 \\
10 \\
28 \\
25 \\
3 \\
5 \\
30 \\
45 \\
18 \\
39 \\
21 \\
13 \\
6 \\
39 \\
17 \\
18 \\
2 \\
46 \\
14 \\
30 \\
38 \\
32 \\
35 \\
3 \\
26 \\
8 \\
99 \\
35 \\
27 \\
49 \\
1 \\
35 \\
32 \\
14 \\
21\end{array}$ \\
\hline
\end{tabular}


Table 2 (oont'd) Age adjusted doath rates per 1,000 population 1940: United States and oach stato. (By place of ocouranoe.)

\begin{tabular}{|c|c|c|}
\hline State & $\begin{array}{l}\text { Age adjusted } \\
\text { death rate }\end{array}$ & Rank \\
\hline 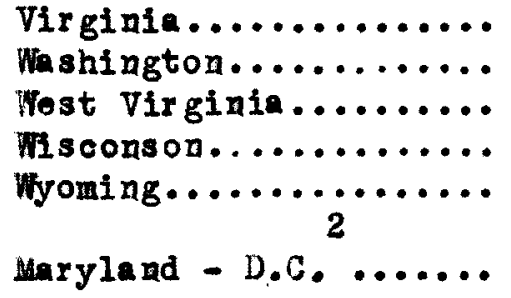 & $\begin{array}{r}12.3 \\
10.0 \\
10.7 \\
9.2 \\
9.8 \\
12.6\end{array}$ & $\begin{array}{r}39 \\
14 \\
29 \\
7 \\
10\end{array}$ \\
\hline
\end{tabular}

1. Age adjusted death rates in the United States 1900-1940. Vital Statistics; Spooial Reports, Soleoted Studies, 23, No. 1, Juno 25, 1945. National Offioe of Vital Statistios, U.S.P.H.S.

2. Exoludes Arlington and Fairfax counties in Virginia. 
Table 3. Infant mortality rates by racos United States and oach state, 1945. (By plaoe of rosidence. Exolusive of still-births rates per 1,000 live births.)

\begin{tabular}{|c|c|c|}
\hline State & White & Nomrhite \\
\hline  & $\begin{array}{l}35.6 \\
38.8 \\
57.7 \\
34.1 \\
31.8 \\
50.8 \\
29.7 \\
33.2 \\
37.0 \\
37.4 \\
36.2 \\
34.1 \\
29.9 \\
34.4 \\
30.1 \\
32.0 \\
45.6 \\
33.2 \\
46.1 \\
32.0 \\
31.3 \\
34.9 \\
30.6 \\
35.9 \\
35.2 \\
31.5 \\
27.2 \\
43.2 \\
36.3 \\
30.1 \\
93.7 \\
30.5 \\
37.5 \\
28.9 \\
35.3 \\
37.3 \\
27.9 \\
36.3 \\
27.3 \\
40.5 \\
27.9 \\
45.2 \\
47.2 \\
30.6\end{array}$ & $\begin{array}{r}57.0 \\
\\
54.6 \\
139.5 \\
32.6 \\
43.9 \\
41.7 \\
39.7 \\
70.9 \\
71.6 \\
61.6 \\
52.7 \\
34.5 \\
52.2 \\
71.6 \\
62.9 \\
59.6 \\
70.4 \\
58.3 \\
122.4 \\
63.4 \\
51.6 \\
50.6 \\
66.7 \\
44.4 \\
64.2 \\
73.1 \\
82.9 \\
85.3 \\
71.4 \\
57.3 \\
179.4 \\
53.1 \\
55.5 \\
45.7 \\
54.6 \\
61.7 \\
60.7 \\
61.1 \\
63.1 \\
61.1 \\
87.5 \\
60.3 \\
59.6 \\
58.4\end{array}$ \\
\hline
\end{tabular}


Table 3 (oont'd). Infant mortality rates by races United States and each state. (By place of residenoe. Exolusive of still-births rates per 1,000 live births.)

\begin{tabular}{|c|c|c|}
\hline State & White & No awhite \\
\hline 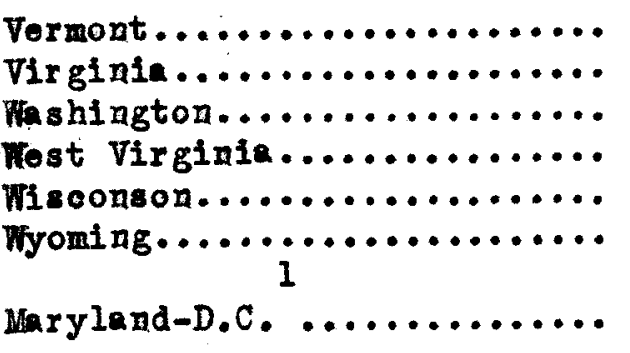 & $\begin{array}{l}34.7 \\
40.3 \\
33.5 \\
51.6 \\
30.7 \\
39.3 \\
33.2\end{array}$ & $\begin{array}{l}0 \\
68.5 \\
65.8 \\
59.4 \\
70.9 \\
53.7 \\
66.6\end{array}$ \\
\hline
\end{tabular}

1. Bxoludos Arlington and Fairfax counties in Virginia.

Sources National office of Vital Stetistics United States Public Health Servioe 
Table 4. Maternal mortality rates by raoe: United States and oach atato, 1946. (By place of residenoe. Rates per 1,000 live births)

\begin{tabular}{|c|c|c|}
\hline State & White & Nownite \\
\hline 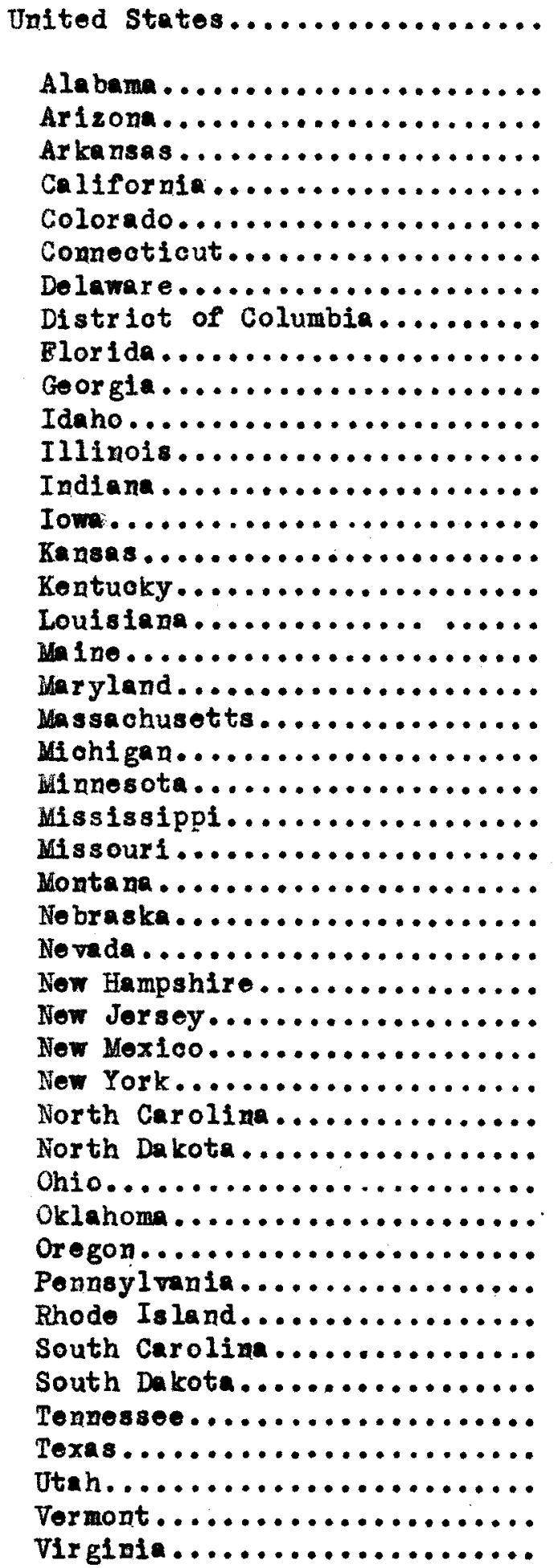 & $\begin{array}{l}1.7 \\
2.4 \\
2.3 \\
2.3 \\
1.6 \\
2.4 \\
1.0 \\
2.8 \\
0.7 \\
1.8 \\
2.1 \\
1.9 \\
1.6 \\
1.6 \\
1.7 \\
1.8 \\
2.4 \\
1.9 \\
2.4 \\
1.3 \\
1.8 \\
1.3 \\
1.4 \\
2.4 \\
2.1 \\
1.6 \\
1.5 \\
1.5 \\
1.8 \\
1.4 \\
3.3 \\
1.6 \\
1.9 \\
1.0 \\
1.6 \\
1.7 \\
1.3 \\
2.0 \\
1.4 \\
1.8 \\
1.2 \\
1.8 \\
1.9 \\
1.3 \\
1.7 \\
1.6\end{array}$ & $\begin{array}{r}4.5 \\
5.1 \\
7.2 \\
4.6 \\
2.2 \\
3.1 \\
6.4 \\
5.5 \\
3.0 \\
6.0 \\
5.3 \\
9.4 \\
3.5 \\
3.5 \\
11.4 \\
3.2 \\
5.9 \\
3.5 \\
20.4 \\
2.3 \\
1.6 \\
3.5 \\
2.7 \\
4.9 \\
4.8 \\
1.6 \\
1.8 \\
4.7 \\
0 \\
4.9 \\
8.7 \\
4.0 \\
4.9 \\
3.0 \\
5.0 \\
6.3 \\
1.7 \\
4.5 \\
3.0 \\
5.4 \\
3.0 \\
5.3 \\
5.0 \\
3.6 \\
30 \\
3.7\end{array}$ \\
\hline
\end{tabular}


Table 4 (oont'd). Maternal nortality rates by raoo: United States and each state, 1945. (By plaoe of residenoe. Rates per 1,000 live births)

\begin{tabular}{|c|c|c|}
\hline State & Whito & Nomwite \\
\hline 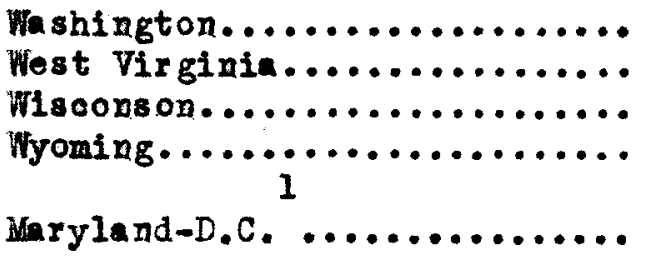 & $\begin{array}{l}1.6 \\
1.7 \\
1.4 \\
1.0 \\
1.2\end{array}$ & $\begin{array}{r}4.3 \\
1.9 \\
0 \\
0 \\
2.6\end{array}$ \\
\hline
\end{tabular}

1. Exoludes Arlington and Fairfax counties in Tirginia.

Source: National offioe of Vital Statistios United States Public Health Service 
Table 5. Percent of live births in hospitalss Uaited States and each state, 1935-1945. (By place of oocurrence, 1935-1941; by place of residence, 1942-1945.)

\begin{tabular}{|c|c|c|c|c|c|c|}
\hline Stato & 1935 & 1937 & 1939 & 1941 & 1943 & 1945 \\
\hline 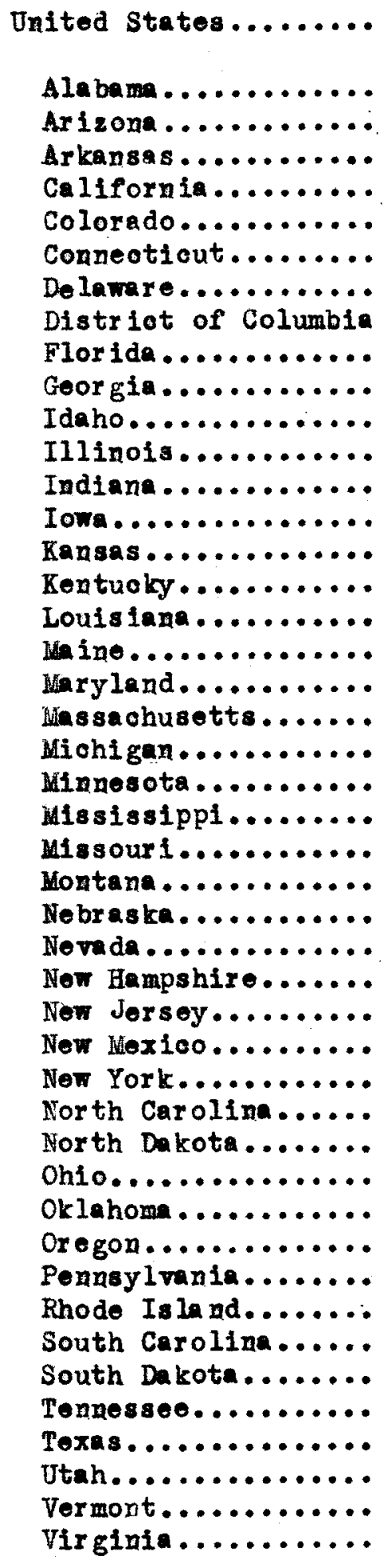 & $\begin{array}{r}36.9 \\
10.6 \\
30.0 \\
5.1 \\
71.6 \\
38.2 \\
74.5 \\
50.0 \\
86.8 \\
23.9 \\
14.1 \\
39.5 \\
56.7 \\
29.6 \\
35.6 \\
31.8 \\
9.3 \\
23.1 \\
27.4 \\
39.6 \\
59.5 \\
39.4 \\
47.8 \\
5.7 \\
32.6 \\
53.6 \\
32.9 \\
51.2 \\
30.6 \\
65.2 \\
12.9 \\
73.6 \\
10.8 \\
34.1 \\
39.4 \\
18.8 \\
61.4 \\
41.3 \\
59.8 \\
7.0 \\
30.5 \\
15.6 \\
20.9 \\
41.1 \\
19.8 \\
15.4\end{array}$ & $\begin{array}{r}12.7 \\
40.0 \\
8.6 \\
78.2 \\
49.6 \\
92.7 \\
57.4 \\
88.6 \\
32.3 \\
18.7 \\
48.4 \\
65.5 \\
39.6 \\
44.1 \\
40.9 \\
12.3 \\
27.5 \\
37.0 \\
47.1 \\
76.6 \\
49.9 \\
56.9 \\
8.1 \\
38.8 \\
62.4 \\
40.0 \\
65.4 \\
26.8 \\
74.1 \\
19.5 \\
79.8 \\
15.5 \\
43.4 \\
49.8 \\
25.9 \\
70.9 \\
51.2 \\
68.8 \\
11.6 \\
40.4 \\
20.4 \\
29.3 \\
55.4 \\
44.5 \\
20.0\end{array}$ & $\begin{array}{l}17.5 \\
52.2 \\
14.2 \\
82.8 \\
55.7 \\
57.9 \\
63.8 \\
89.9 \\
38.5 \\
24.3 \\
55.3 \\
69.5 \\
46.6 \\
52.7 \\
47.2 \\
15.6 \\
36.3 \\
43.2 \\
54.6 \\
81.5 \\
57.2 \\
63.9 \\
11.6 \\
44.7 \\
72.6 \\
47.7 \\
75.5 \\
70.8 \\
80.4 \\
25.2 \\
54.6 \\
21.2 \\
54.2 \\
57.1 \\
31.8 \\
78.5 \\
57.7 \\
75.3 \\
16.0 \\
51.0 \\
25.1 \\
37.5 \\
62.2 \\
50.7 \\
24.9\end{array}$ & $\begin{array}{l}24.6 \\
68.8 \\
20.8 \\
89.3 \\
66.2 \\
94.0 \\
73.5 \\
92.5 \\
50.4 \\
43.4 \\
71.5 \\
79.1 \\
59.3 \\
64.4 \\
59.6 \\
22.5 \\
48.2 \\
57.7 \\
61.9 \\
88.4 \\
71.7 \\
72.8 \\
17.2 \\
53.2 \\
84.7 \\
61.2 \\
87.4 \\
81.8 \\
87.7 \\
32.4 \\
89.6 \\
30.7 \\
64.3 \\
68.8 \\
45.9 \\
90.1 \\
68.1 \\
83.1 \\
23.2 \\
60.5 \\
33.4 \\
48.0 \\
74.4 \\
63.1 \\
34.7\end{array}$ & $\begin{array}{l}34.5 \\
77.5 \\
35.0 \\
93.8 \\
77.5 \\
97.3 \\
81.1 \\
92.9 \\
61.8 \\
45.5 \\
87.6 \\
87.6 \\
75.7 \\
79.0 \\
78.2 \\
33.3 \\
54.5 \\
75.9 \\
70.7 \\
93.8 \\
84.3 \\
85.6 \\
26.6 \\
64.3 \\
91.4 \\
78.8 \\
92.5 \\
89.9 \\
91.0 \\
50.2 \\
93.7 \\
45.7 \\
81.2 \\
80.4 \\
61.3 \\
95.6 \\
77.9 \\
89.2 \\
35.7 \\
77.0 \\
42.2 \\
61.7 \\
87.5 \\
75.0 \\
50.7\end{array}$ & $\begin{array}{l}43.1 \\
81.6 \\
46.6 \\
96.1 \\
85.2 \\
98.4 \\
85.8 \\
95.4 \\
70.3 \\
53.0 \\
94.8 \\
91.9 \\
84.1 \\
88.8 \\
87.6 \\
43.8 \\
65.8 \\
83.8 \\
77.9 \\
96.3 \\
91.2 \\
92.9 \\
32.9 \\
73.8 \\
95.1 \\
87.7 \\
96.2 \\
95.1 \\
94.0 \\
58.0 \\
96.1 \\
55.2 \\
58.3 \\
57.6 \\
72.4 \\
98.0 \\
85.0 \\
94.5 \\
44.7 \\
86.4 \\
53.1 \\
68.7 \\
94.1 \\
85.2 \\
60.7\end{array}$ \\
\hline
\end{tabular}


Table 5 (oont'd). Peroent of live births in hospitals: United States and each state, 1935-1945. (By place of oocurenoe, 1935-1941; by plaoe of residenoe, 1942-1945.)

\begin{tabular}{c|r|r|r|r|r|c}
\hline State & 1935 & 1937 & 1939 & 1941 & 1943 & 1945 \\
\hline Washington......... & 65.3 & 75.6 & 83.0 & 90.9 & 96.3 & 97.5 \\
West Virginia...... & 9.1 & 12.5 & 16.3 & 24.7 & 38.1 & $\mathbf{4 6 . 2}$ \\
Wisconson.......... & 38.0 & 47.5 & 53.5 & 70.5 & 83.3 & 91.1 \\
Wyoming........... & 13.8 & 10.2 & 58.5 & 76.1 & 87.9 & 91.5 \\
Miaryland-D.C...... & 53.0 & 59.9 & 66.3 & 72.5 & 76.3 & 82.7 \\
\hline
\end{tabular}

1. Exoludes Arlington and Fairfax counties in Virginta.

Source: Hational Offioe of Vital statistios United States Public Health Service 
Table 6. Percent live births, in hospitals by county gxoup: United States and each state, 1945. ${ }^{1}$ (By place of residonce)

\begin{tabular}{|c|c|c|c|c|c|c|c|c|}
\hline Stato & 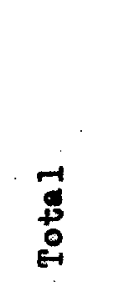 & 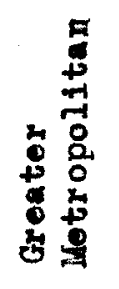 & 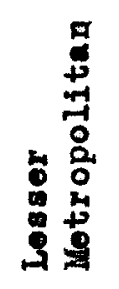 & 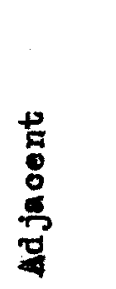 & 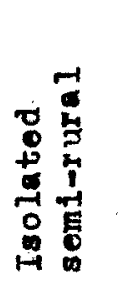 & 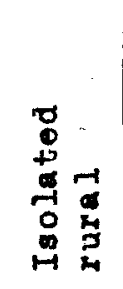 & 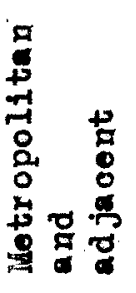 & $\begin{array}{c}8 \\
8 \\
+ \\
5 \\
0 \\
0 \\
0 \\
H\end{array}$ \\
\hline 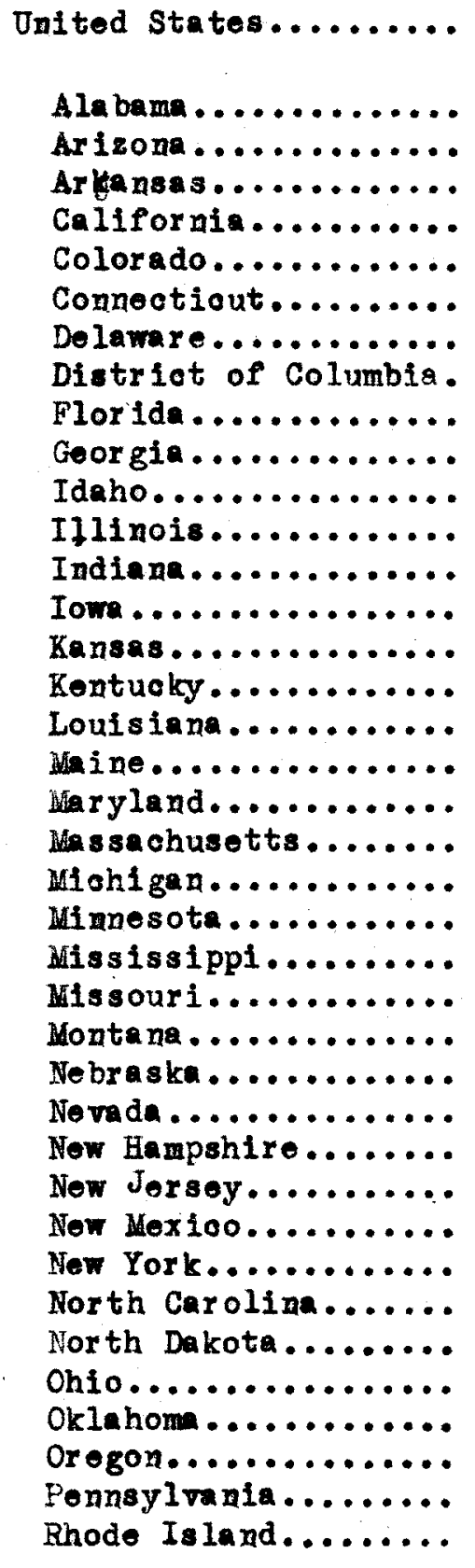 & $\begin{array}{l}43.1 \\
81.6 \\
46.6 \\
96.1 \\
85.2 \\
98.4 \\
85.8 \\
95.4 \\
70.3 \\
53.0 \\
94.8 \\
91.9 \\
84.1 \\
88.8 \\
87.6 \\
43.8 \\
65.8 \\
83.8 \\
77.9 \\
96.3 \\
91.2 \\
92.9 \\
32.9 \\
73.8 \\
95.1 \\
57.7 \\
96.2 \\
95.1 \\
94.0 \\
58.0 \\
96.1 \\
55.2 \\
88.3 \\
87.6 \\
72.4 \\
99.0 \\
85.0 \\
94.5\end{array}$ & $\begin{array}{l}82.4 \\
97.3 \\
93.5 \\
93.3\end{array}$ & $\begin{array}{l}65.2 \\
86.9 \\
80.7 \\
96.3 \\
90.9 \\
98.5 \\
95.3 \\
\\
95.4 \\
83.1 \\
96.0 \\
87.0 \\
94.5 \\
92.1 \\
84.8 \\
89.6 \\
96.0 \\
94.5 \\
94.4 \\
97.9 \\
49.6 \\
91.3 \\
95.9 \\
96.9 \\
91.0 \\
95.9 \\
83.8 \\
92.7 \\
89.3 \\
99.9 \\
81.2 \\
94.3\end{array}$ & $\begin{array}{l}33.3 \\
73.8 \\
38.9 \\
94.1 \\
85.2 \\
97.9 \\
69.6 \\
\\
64.6 \\
43.2 \\
95.3 \\
90.3 \\
79.7 \\
86.5 \\
89.4 \\
41.6 \\
51.7 \\
89.9 \\
66.8 \\
94.9 \\
89.3 \\
83.7 \\
28.2 \\
59.7 \\
. \\
90.3 \\
95.5 \\
89.5 \\
36.9 \\
92.0 \\
54.4 \\
79.1 \\
68.6 \\
99.2 \\
76.8 \\
97.6\end{array}$ & $\begin{array}{l}40.6 \\
78.6 \\
46.1 \\
93.3 \\
77.7 \\
59.7 \\
\\
63.1 \\
46.3 \\
96.1 \\
81.3 \\
85.1 \\
89.7 \\
87.4 \\
38.9 \\
57.1 \\
75.8 \\
70.3 \\
88.9 \\
84.4 \\
90.2 \\
37.1 \\
59.5 \\
96.6 \\
86.7 \\
97.3 \\
92.7 \\
65.9 \\
88.8 \\
48.1 \\
94.7 \\
79.1 \\
69.9 \\
97.2 \\
79.4\end{array}$ & $\begin{array}{l} \\
42.6 \\
29.0 \\
39.0 \\
56.0 \\
53.9 \\
75.3 \\
77.7 \\
20.8 \\
39.7 \\
79.8 \\
54.4 \\
93.3 \\
66.9 \\
86.9 \\
25.5 \\
38.9 \\
91.8 \\
79.0 \\
93.5 \\
95.8 \\
\\
40.3 \\
32.4 \\
83.1 \\
45.2 \\
50.0 \\
95.3 \\
46.0\end{array}$ & $\begin{array}{l}52.0 \\
84.9 \\
56.2 \\
96.3 \\
89.5 \\
98.4 \\
92.0 \\
95.4 \\
90.7 \\
69.7 \\
95.3 \\
94.4 \\
85.3 \\
90.5 \\
91.0 \\
73.0 \\
80.3 \\
92.6 \\
80.2 \\
96.4 \\
92.6 \\
96.7 \\
33.7 \\
88.2 \\
94.1 \\
96.1 \\
94.0 \\
36.9 \\
96.6 \\
67.3 \\
89.8 \\
80.3 \\
99.8 \\
85.5 \\
94.5\end{array}$ & $\begin{array}{l}61.2 \\
34.0 \\
74.7 \\
43.6 \\
93.6 \\
77.7 \\
59.7 \\
59.2 \\
39.8 \\
94.7 \\
79.1 \\
81.9 \\
86.9 \\
84.4 \\
30.7 \\
53.9 \\
76.2 \\
66.5 \\
89.7 \\
80.2 \\
89.4 \\
32.7 \\
53.1 \\
95.1 \\
83.3 \\
96.2 \\
93.0 \\
59.8 \\
88.8 \\
43.8 \\
88.3 \\
76.1 \\
66.5 \\
97.0 \\
77.1\end{array}$ \\
\hline
\end{tabular}


Table 6 (oont'd). Pereont live births in hospitals by oounty group: Unitod States and oach stato, 1945. (By place of residonoo)

\begin{tabular}{|c|c|c|c|c|c|c|c|c|}
\hline State & 孪 & 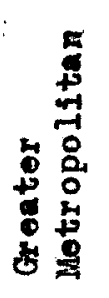 & 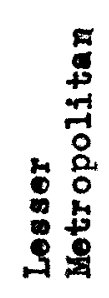 & $\begin{array}{l}\stackrel{8}{8} \\
0 \\
8 \\
0 \\
0 \\
8\end{array}$ & 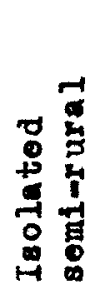 & 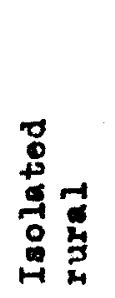 & 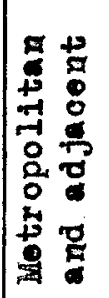 & $\begin{array}{l}0 \\
0 \\
+0 \\
0 \\
0 \\
0 \\
0 \\
0 \\
-1\end{array}$ \\
\hline South Carolina........ & 44.7 & & 67.5 & 38.5 & 44.5 & 10.6 & 50.4 & 41.0 \\
\hline 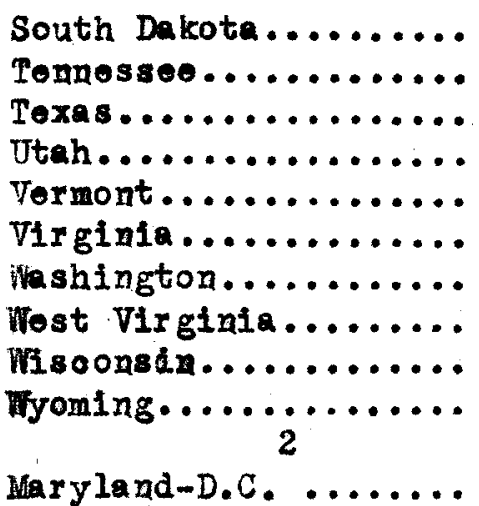 & $\begin{array}{l}86.4 \\
53.1 \\
68.7 \\
94.1 \\
85.2 \\
60.7 \\
97.5 \\
46.2 \\
91.1 \\
91.5 \\
83.6\end{array}$ & 87.7 & $\begin{array}{l}77.3 \\
82.8 \\
97.4 \\
\\
91.0 \\
98.8 \\
75.7 \\
96.0\end{array}$ & $\begin{array}{l}89.1 \\
43.7 \\
59.5 \\
95.4 \\
96.3 \\
57.6 \\
96.6 \\
31.0 \\
91.6\end{array}$ & $\begin{array}{l}93.6 \\
46.4 \\
60.6 \\
92.1 \\
85.7 \\
51.4 \\
95.8 \\
43.0 \\
88.2 \\
91.1 \\
70.3\end{array}$ & $\begin{array}{l}79.0 \\
26.5 \\
50.1 \\
75.7 \\
82.1 \\
36.2 \\
94.2 \\
30.8 \\
78.8 \\
92.4 \\
54.4\end{array}$ & $\begin{array}{l}89.1 \\
65.5 \\
76.5 \\
96.7 \\
86.3 \\
78.5 \\
98.1 \\
53.6 \\
94.5\end{array}$ & $\begin{array}{l}86.4 \\
39.3 \\
59.1 \\
83.5 \\
85.1 \\
44.9 \\
95.6 \\
40.2 \\
87.3 \\
91.5 \\
66.5\end{array}$ \\
\hline
\end{tabular}

1. Vital Statistios of the Unitod States, 1945. Part II. National Offioe of Vital Statistios.

2. Inoludes Arlington and Pairfax oountios in Virginia. 
Table 7. Peroent of 1170 births in hospitals, by race; Unitod States and oach stato. 1935, 1939, 1941, and 1945 .

(By place of ocourence 1935, 1939, and 1941; by place of residenoe 1945)

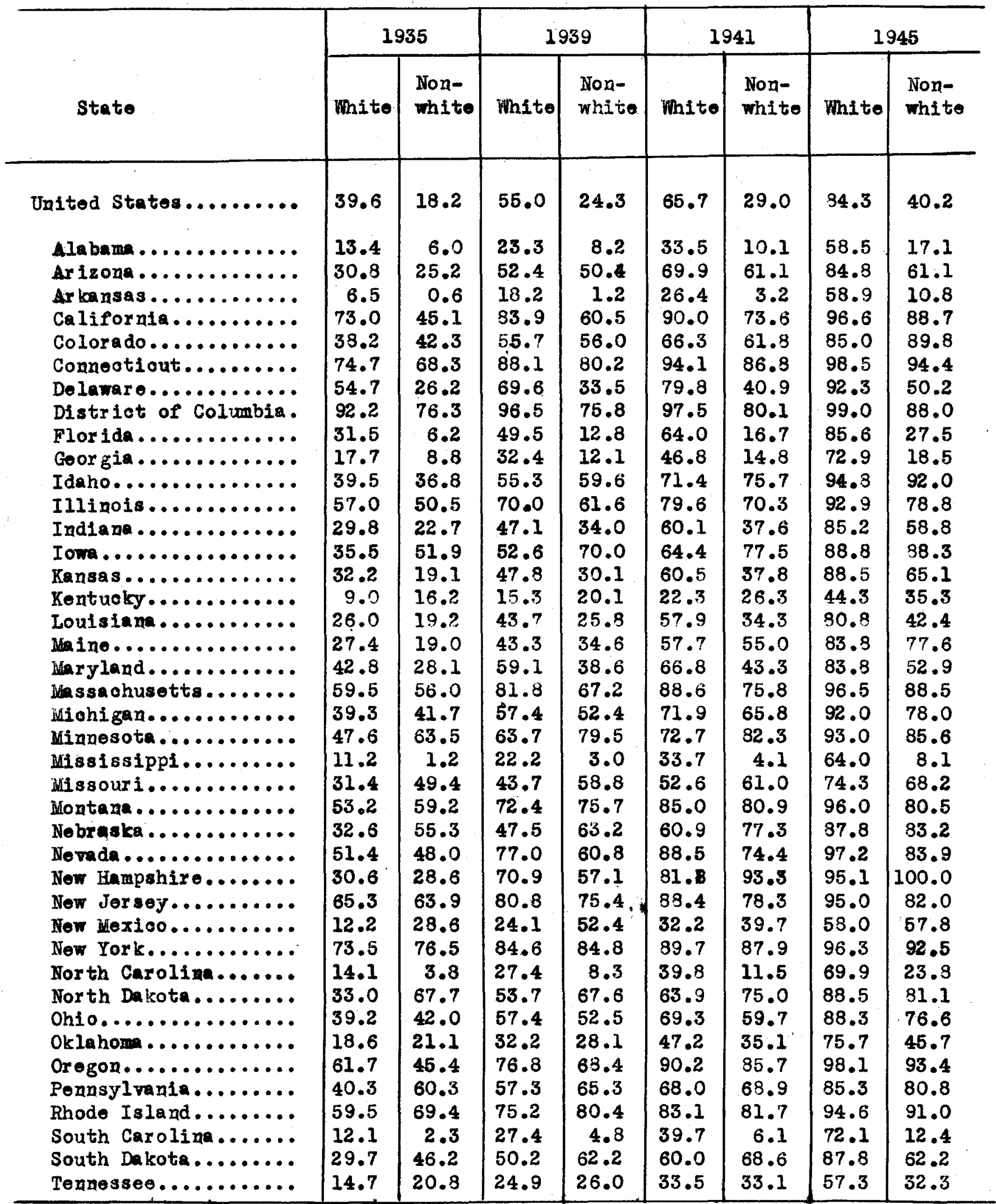


Table 7 (oont'd). Peroent of live births in hospltals, by race; United States and oach state. 1935, 1939, 1941 and 1945.

(By place of oocurence 1935, 1939, and 1941; by place of residence 1945.)

\begin{tabular}{|c|c|c|c|c|c|c|c|c|}
\hline \multirow[b]{2}{*}{ State } & \multicolumn{2}{|c|}{1935} & \multicolumn{2}{|c|}{1939} & \multicolumn{2}{|c|}{1941} & \multicolumn{2}{|c|}{1945} \\
\hline & White & $\begin{array}{l}\text { Non- } \\
\text { whito }\end{array}$ & Whito & $\begin{array}{l}\text { Non- } \\
\text { white }\end{array}$ & Whito & $\begin{array}{l}\text { Non- } \\
\text { white }\end{array}$ & White & $\begin{array}{l}\text { Non- } \\
\text { white }\end{array}$ \\
\hline 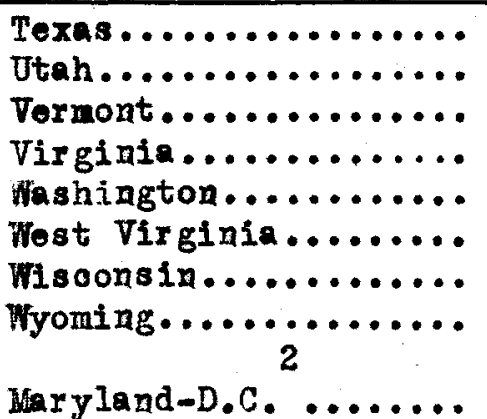 & $\begin{array}{r}22.0 \\
41.0 \\
19.8 \\
19.5 \\
66.3 \\
9.3 \\
37.8 \\
14.0 \\
55.2\end{array}$ & $\begin{array}{r}12.8 \\
49.1 \\
-- \\
5.4 \\
31.3 \\
4.8 \\
57.6 \\
6.7 \\
46.5\end{array}$ & $\begin{array}{l}40.0 \\
62.5 \\
50.7 \\
31.1 \\
83.6 \\
16.9 \\
53.3 \\
53.4 \\
70.4\end{array}$ & $\begin{array}{r}19.8 \\
73.6 \\
-- \\
9.5 \\
59.2 \\
6.6 \\
71.8 \\
63.9 \\
54.3\end{array}$ & $\begin{array}{l}51.5 \\
74.4 \\
63.1 \\
42.9 \\
91.3 \\
25.2 \\
70.4 \\
76.1 \\
76.7\end{array}$ & $\begin{array}{l}24.3 \\
75.4 \\
66.7 \\
12.9 \\
74.4 \\
12.3 \\
73.6 \\
76.4 \\
53.9\end{array}$ & $\begin{array}{l}72.7 \\
94.1 \\
85.2 \\
71.8 \\
97.6 \\
47.6 \\
91.3 \\
91.7 \\
87.4\end{array}$ & $\begin{array}{r}40.5 \\
96.7 \\
100.0 \\
27.6 \\
92.8 \\
23.2 \\
79.1 \\
86.4 \\
66.7\end{array}$ \\
\hline
\end{tabular}

1. National Office of Vital Statistics, United States Publio Hoalth Sorvioo. 2. Exoludes Arlington and Fairfax counties in Virginia. 
,

-

$\cdot$

$\therefore$

APPENDIX C 


\section{ACADEMY OF PEDIATRICS STUDY OF CHILD HEALTH SERVICES}

\section{Schedule 1-A. Information on Pediatric Hospitals, General Hospitals and Other Hospitals with Maternity Facilities}

Date

Code No.

1. NAME AND LOCATION OF HOSPITAL

a. Name

Street and number

City or town

b. Data for year ended

County

State

(Same year to be used for all annual data.)

2. TYPE OF OWNERSHIP OR CONTROL (Check one only.)

\section{Governmental}

Non-Governmental
(1) Federal
(2) State
(3) City, town, village or other municipality
(4) County
(5) City-County

Non-profit

(6) Church

(7) Non profit association

(11) Other (Explain)

3. TYPE OF HOSPITAL (Check one only.)
(1) General, incl. EEN \& $\mathrm{T}$
(2) Maternity
(3) Pediatric

4. AMA APPROVAL (Check)

American Medical Association Registry: Yes $\square$ No

5. MEDICAL SCHOOL AFFILIATION

Yes No

a. Is the hospital affiliated with a medical school?

b. If YES, check purpose:

(1) Instruction undergraduate students

(2) Postgraduate instruction

(3) Research and investigation

6. RESTRICTIONS AS TO RACE OF PATIENT

Check the race of the patients admitted:
(1) White only
(2) Nonwhite only
(3) White and nonwhite

7. ADMINISTRATOR

Check type of training (one only)
(1) Medical doctor
(2) Clergyman
(3) Osteopath
(4) Chiropractor

(5) Reg. or grad. nurse: Nun, Sister, Mother

(6) Reg. or grad. nurse: Not Nun, Sister, Mother

(7) Nun, Sister or Mother: Not reg. or grad. nurse

(8) Other (specify)

8. ADMISSION OF CHILDREN

a. Are children entirely separated from adult patients (that is, are permanent facilities set aside for exclusive use of children)?

Yes $\square \quad$ No

If No, what provisions are there for children?

If Yes;

(1) Are there separate wards for infants (other than newborn)?

Yes $\square \quad$ No

If Yes, check upper age limit: (a) 1 year

(b) 18 months

(c) 2 years

(2) What is the upper age limit for admission to children's department? _._ _ _ years

(3) Is there separation of nonwhite from other children?

Yes $\square$ No

b. Is isolation and/or cubicle separation procedure followed for new admissions? Yes $\square$ No

If YES, check whether for (1) $24 \mathrm{hrs.}$

(2) 48 hours.

(3) Longer

c. When contagious diseases develop after patients are admitted: (check)
(1) Are they retained under isolation?
(2) Transferred elsewhere? 
9. SUMMARY OF SERVICES RENDERED

Number of patients (exclude newborn)

(1) Remaining in hospital beginning of ye

(2) Admitted (exclude births)

(3) Total treated (1 and 2)

(4) Discharged (except deaths)

(5) Died (exclude stillborn)

(6) Total discharged and died (4 and 5)

(7) Remaining end of year (3 minus 6)

*Specify upper age limit of children: thru
All Children only* Patients All Nonwhite

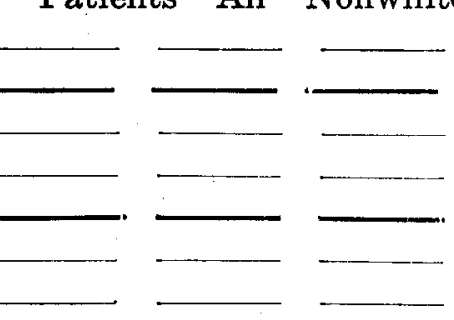

10. TOTAL PATIENT DAYS OF SERVICE RENDERED (exclude newborn)
(1) All patients
(2) All children
(3) Nonwhite children

11. AUTOPSIES: (1) Number by permission

(2) Stillbirths

12. NEWBORN

a. Number of infants, including prematures, born in this hospital:

(1) Births (excluding stillborn)

(2) Stillborn

(3) Deaths (excluding stillborn)

(4) Newborn (live babies) discharged (1 minus 3)

b. Days of care to newborn

\section{Physical Plant}

13. BEDS FOR PATIENTS, BY TYPE OF BEDROOM Type of bedroom

(1) One person

(2) Two

(3) Three or four

(4) Five and over

(5) Total

Note: Give the present number of beds actually set up for in-patient care, including cribs and bassinets in pediatric department (exclude bassinets in nursery and beds in emergency and labor rooms.)

14. BEDS USED FOR TEACHING MEDICAL STUDENTS

Of the beds allotted to children only (item 13 (5), last column), how many are regularly used for teaching?

15. FLOOR AREA

Floor area in bedrooms for children (exclude corridors and bathrooms) sq. ft.

16. NURSERY ACCOMMODATIONS

List each nursery separately.

(1) Full-term:

Well

"

9

Sick or suspect (if separate from well)

(2) Premature (if separate from full-term) :

Well

Sick or suspect (if separate from well)

(3) Total (1 plus 2)

17. OXYGEN TENTS

Number of oxygen tents available in hospital for use with children
Number of beds All patients Children only.

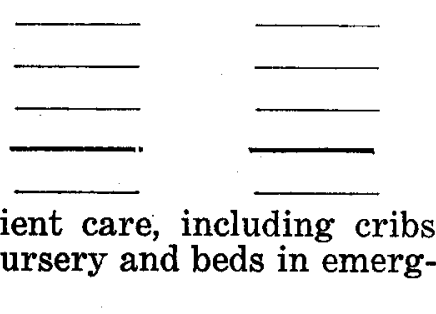




\section{Medical Staff}

18. STAFF MEMBERS (exclusive of house staff)

Which of the following hospital staff members is responsible for the care of children other than private patients* (check one or more)
$\square$ (1) Pediatrician, full-time
(2) Pediatrician, part-time
(3) Other specialist
(4) General practitioner

* Check here if only private patients are admitted

19. HOUSE STAFF

a. Is pediatric service covered by house staff assigned to that service only? Yes $\square$ No If No, indicate other duties

b. Total number of house staff employed by hospital

c. Number of staff members on pediatrics at any time

d. Usual length of service on pediatrics (check) :

(1) 1 month or less

(2) 2 months but less than 3

(3) 3 months but less than 6

(4) 6 months but less than 9

(5) 9 months but less than 12

(6) 1 year or longer

Nursing Staff

\begin{tabular}{|c|c|c|c|}
\hline $\begin{array}{l}\text { Interns } \\
\text { (1st yr.) }\end{array}$ & $\begin{array}{l}\text { Ass't. } \\
\text { Residents } \\
\text { (2nd yr.) }\end{array}$ & $\begin{array}{l}\text { Residents } \\
\text { (3rd yr. } \\
\text { or longer) }\end{array}$ & $\begin{array}{r}\text { Others } \\
\text { (explain) }\end{array}$ \\
\hline & & & \\
\hline
\end{tabular}
Ass't. Residents Others
Residents (3rd yr. (explain)
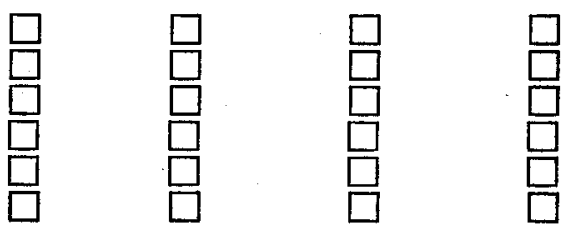

20. NURSES IN ATTENDANCE

Is there a graduate nurse on duty at all times

(1) In newborn nursery?

(2) In premature nursery?

(3) In pediatric unit?

21. TRAINING IN PEDIATRICS

Has nursing supervisor of specified unit had postgraduate training in pediatrics:

(1) Newborn nursery?

(2) Premature nursery?

(3) Pediatric unit?

22. NURSING SERVICE

a. Do graduate nurses administer intravenous therapy?

b. Do student nurses administer intravenous therapy?

23. NURSING SCHOOL

Is there a nursing school?

Other Departmental Functions

24. DEPARTMENTS AND SERVICES IN HOSPITAL

a. Is medical social service organized as regular department of hospital?

b. Is there X-ray service in the hospital?

If YES, check types of service available

(1) Diagnosis

(2) X-ray therapy

c. Is there a clinical laboratory in the hospital?

If YES, check types of service available:
(1) Bacteriology
(2) Biochemistry
(3) Hematology
Yes No

d. Is there a department of physical therapy?

e. Is there a department of occupational therapy?

f. Is there a dental service (other than emergency) in the hospital?

(4) Basal metabolism

(5) Electrocardiology

(6) Electroencephalography --

If YES, check types of service provided for children:

(1) Extractions

(2) Fillings

(3) Prophylaxis

(4) Orthodontia 
25. DIETARY DEPARTMENT

a. Is person in charge a graduate of a college course in home economics?

b. Has she had a hospital internship in dietetics?

26. OUT-PATIENT DEPARTMENT

a. Is there an out-patient department?

If YES -

b. Is there a general pediatric clinic separate from clinics for adults?

c. Check types of service provided to children

(1) General medical

(2) Allergy

(3) Cardiology

(4) Mental hygiene

(5) Luetic

(6) Neurology

(7) Surgery

(8) Eye

(9) Ear, nose and throat

(10) Orthopedic

(11) Dentistry

In separate

In

Pediatric clinic Adult clinic

d. Number of visits during report year

(1) Total visits, all ages

(3) Visits to pediatric clinic only

(2) Total visits by children

Data for Pediatric Hospitals and General Hospitals with 25 or More Pediatric Beds

27. TYPE OF SERVICE IN PEDIATRICS

(Exclude newborn.)

Children discharged

\author{
(Exclude newborn.)
}

(1) General medical

(2) Surgical including orthopedic and EEN \& T

(3) Contagious

(a) Acute poliomyelitis

(4) Other

(5) Total (1 thru 4, excl. 3a)

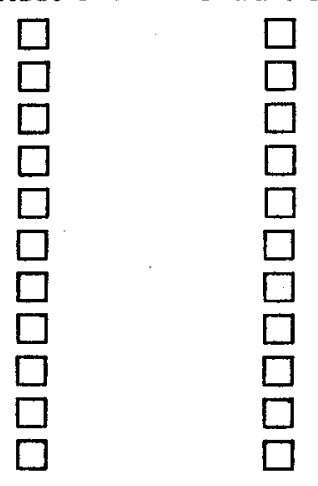

* The total should be the same as item $9(6)$, children only

28. BED ALLOTMENTS FOR CHILDREN ACCORDING TO TYPES OF SERVICE

(1) General medical

(2) Surgical including orthopedic and EEN \& T

(3) Beds for infants other than newborn if not assigned under (1) or (2)

(4) Contagious

(5) All others; unassigned

(6) Total (1 thru 5)

$\frac{\text { (include died) }}{\text { All Nonwhite }}$

* The total should be the same as item $13(5)$, last column.

29. BED ALLOTMENTS FOR CHILDREN BY RACE

Number of beds for (1) white children only ___ $\quad$ (2) nonwhite children only

Name of field agent:

Return this schedule to: 
ACAOEMY OF PEDIATRICS STUDY OF CHILD HEALTH SERVICES

Seneoule 1-B。 Information on Peolatric Hospitals, general Hospitals amo Otwer Hospitals with Materuitr facilities

$(h-1)$ I. NAME AND LCCATION OF hOSPITAL

A. NAME

Stare

Citr OR TOWK

- Date of repont

194

G. OATE FOR YEAR EMOEO

STREe AMO NUMaER

TOWHenip

Peolathic Hospitals: Fill in susequent oata for "AlL Patients", writing "game" where onta ron chilonel onir ane requesteo. Othen Hospitals: Fill in sussequent oxta fon "All Patients" ane if possiole for "Chilonen Only" where requesteo; If outa fon chilonen are estimateo, makx "estimate".

Where. Negro chlleren ane sepanateo from otmer chilceken, fill in suesequent oata for "All Chiloren" amo "Negro Chilonen omlr". IF mo separation of chileaen or nace is maoe, estimate oata pon Negao chiconen If possible amo manix "Estimate".

(F2is)

2. AOHISSION OF CHILOREN

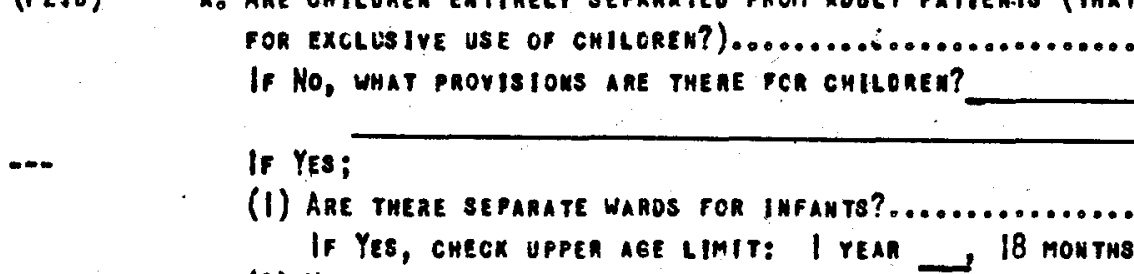

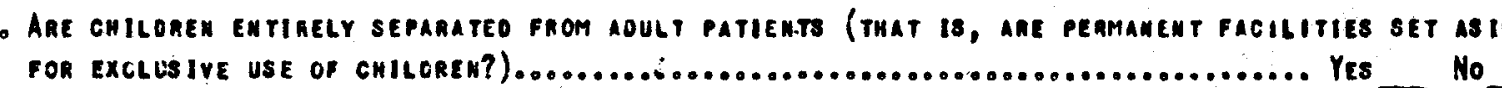

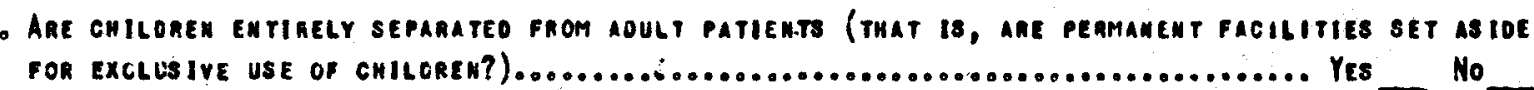

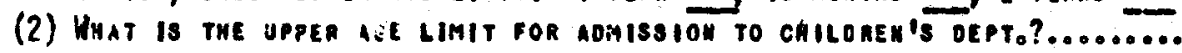

(3) Is there separation OF NeERO FRCH OtMER EMILOREM?.

Yes No No.

\section{Patient Seavice Data for One Year}

$(0-\mid x)$ 3. SUMMARY OF SERVICES RENDERED

NumeER of Patieuts:

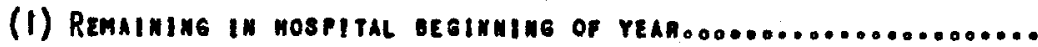

(2) ADMjtTEO (ExcLUdE olRths) ..................................

(3) Total treateo (I amo 2).

(4) Discharged (excert deaths)

(5) Dieo (exclude stilloiaths).

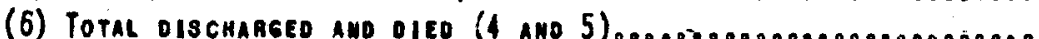

(7) RemalMing EnO of VEAR...........................................

rifars

- Specirr uppen aee limit of chilonen:

$(0-2 x)$ 4. TOTAL PATIENT DAYS OF SERVICE RENDERED (excLUOE HIwU ORN)

(1) ALL PATIENTS (2) ALI CMILOREN

-.. 5. TYFE OF SERVICE IN PEDIATRICS

(EXCLUDE NEWrONR)

(1) General medicalo............................

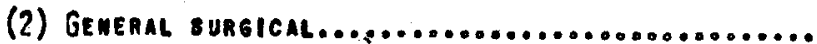

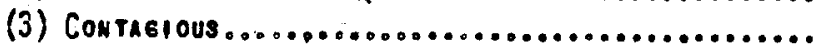

(a) ACUTE POL IOMYELITIS.

(4) Onthopedic...................................

(A) Chronic pollomrELITIS......................

(5) EYT, EAR, WOSE AMD THROATa....................

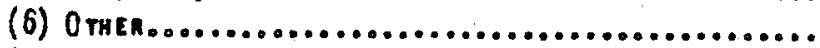

(7) TOTAL (I THAU 6 , EXCL, 3A AMO 4A).

- The total shoulo oe the same as item 3 (6). . . The total shoulo de the same as item 4.

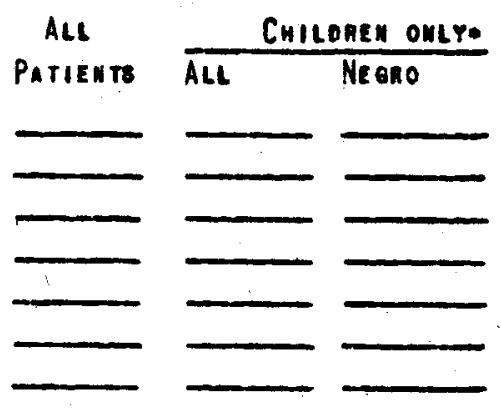

(3) NeGro CHILCREN No. chilgren disCMAREE No. patient oaye

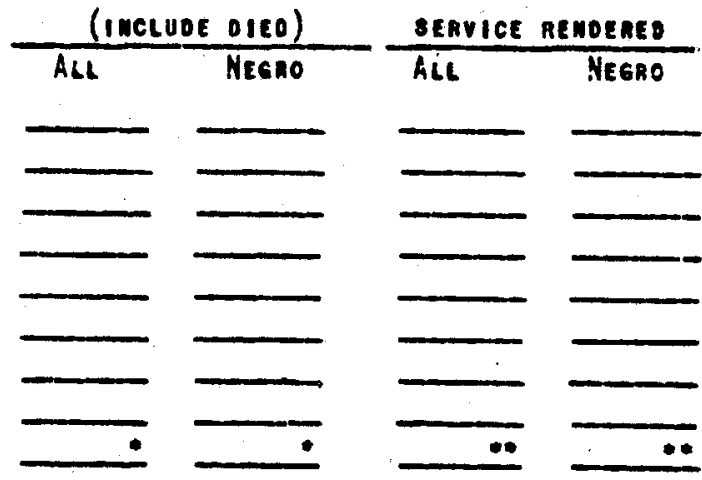
YeAns Ko 


\section{Phrsical Plant}

(C-6x) 6. ROOMS ANC BEOS FOR PATIENTS, BY TYPE OF BEOROOM

TrPe of geonoom

(1) OWE PEnSDN.

(2) Two.......................................

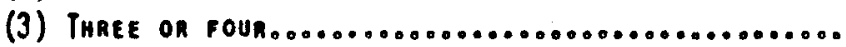

(4) Five and oren....................................

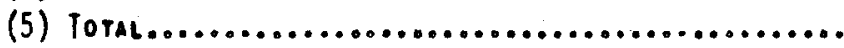

ALL PATIENTS

Ho. ROOHS NO. BEOS

Chiloren onLY

NO. aOOMS HO. BEDS
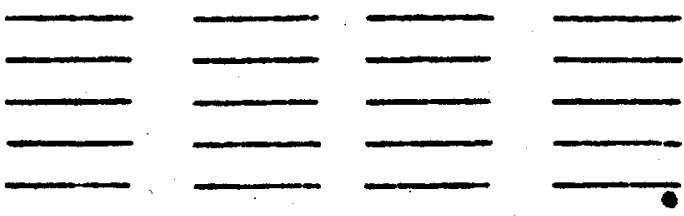

- Note: Give the present mumaer of beds actually set up for im-patient care, incluoine chilloren's

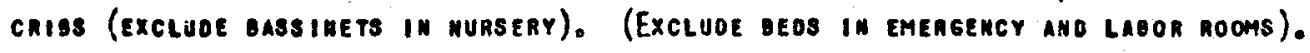

(C-9x) 7. BED ALLOTMENTS FCR CHILOREN ACCORDING TO TYPE OF SERVICE

IF A SEPARATE oEPAATHEMT -

ALL CHILOEE

NEGRO ONLY

(Т) GEMERAL MEOIGAL ........................................

(2) Genenal suncical.............................................

(3) Beos con infants if not unoen (1) on (2).......................

(4) Contagious.

(5) OATHOPEOIC.

(6) EYE, eAh, MOSE ano thrOAT.

(7) Orher; including unassigneo

(8) Total (1 thaough 7). - The total should oe the same as item 6 (5), last columio

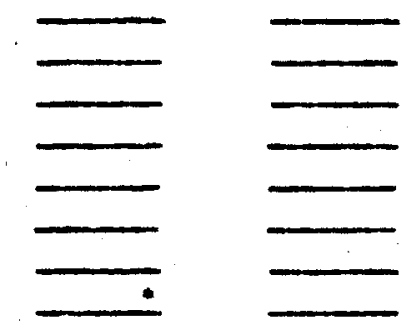

8. BEDS USED FOR TEACHING MEDICAL STUDENTS

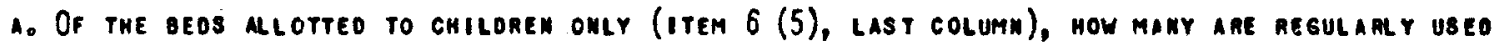
FOR TEACHING?

$(C-10 x)$ 9. FLOOR AREA

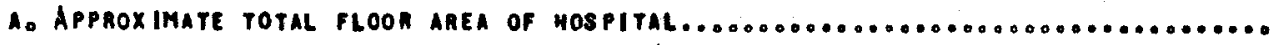

B. Flcor area in bedrooms for children - (exclude cormioors amo oathrooms)....... SO. FT. 8Q. F T

(C-8x, 10. NURSERY ACCOHHODATIONS

- $-10 x$ ) LIJT EACM NURSERY SEPARATELY.

(1) FULL-TEnM:

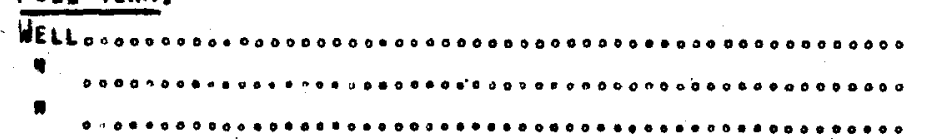

SIAK OR SUSPECT (IF SEPARATE PROM WELL)

Numeea of Numger of Floor area oAssinETS inCUBATORS (SO.FT.)

(2) Premature (1F sepahate from full-team):

Hell....................................................

Sigk on suspect (IF SEPARATE FROM WELL)

(3) TOTAL (1 PLUS 2).

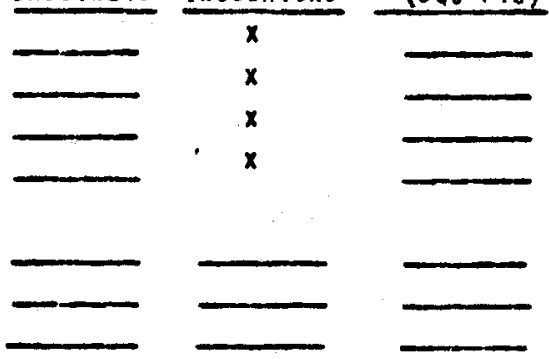

11. OXYGEN TENTS

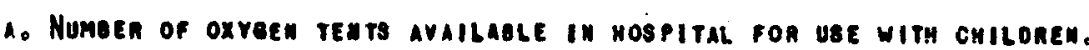




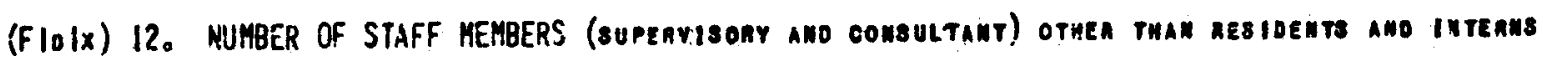

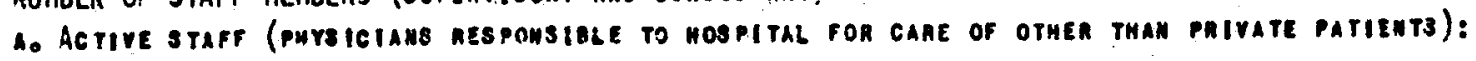

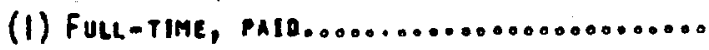

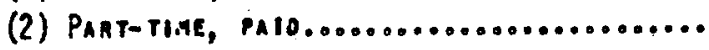

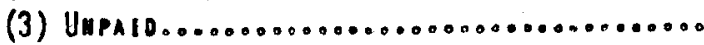

9. Courtesr stafe (phrsicians in practice allowed to care fon onn chilo patients in

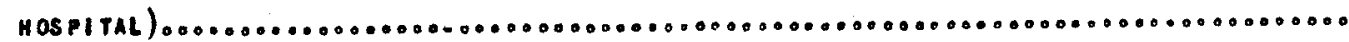

-.- 13. NUMBER OF STAFF MEMBERS AVAILABLE FOR CHILOREN:

A. Active otheF:

\section{Peoiataicians}

Diplomates: Nolloiplomates

(1) FuLl-tume, Pa10.0.....................0.0

(2) PART-TIME, PAL10.......................

(3) UnPA10.........0.........................

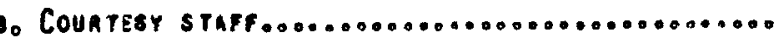

Genenal Practitionens

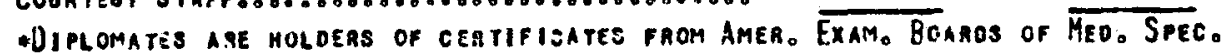

\section{House STAfF on PEDIATRIEs}

14. PEDIATRIC SERVICE

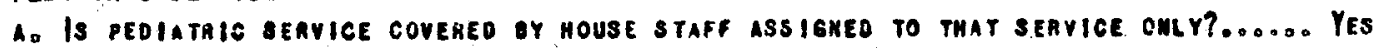
iF NO, IHOICATE OTMER OUTIES

(1) TOTAL number of hOUSE STAFF

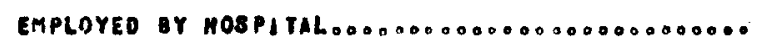

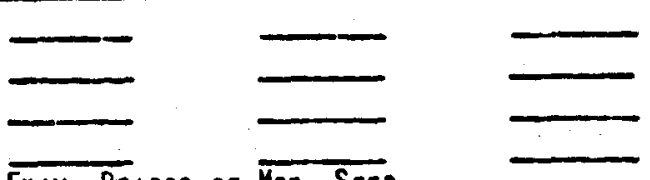

(2) Number of s tafF memsias on

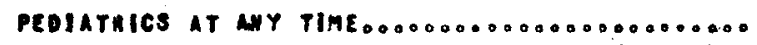

(3) USUAL LENGTH OF SERYIEE ON PEOIATRIES (CHECK):

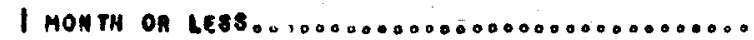

2 MONTHS But LESS than 3.........................

3 mon rys out less thas 6 o.......................

6 MONTHS out less than $9 . \ldots . . . . \ldots \ldots . . . . . . . .$.

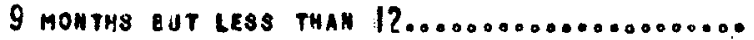

I YeAR OR LONGER.

NuRs InG STAFF

... 16. NURSES IN ATTENDANCE

IS tHERE a gaAOUATE nUASE ON DUTY -

(1) IN WEWBORM WUASERY?.

(2) In PRematuke Unit?

(3) In PEOIATRIC UNIr?

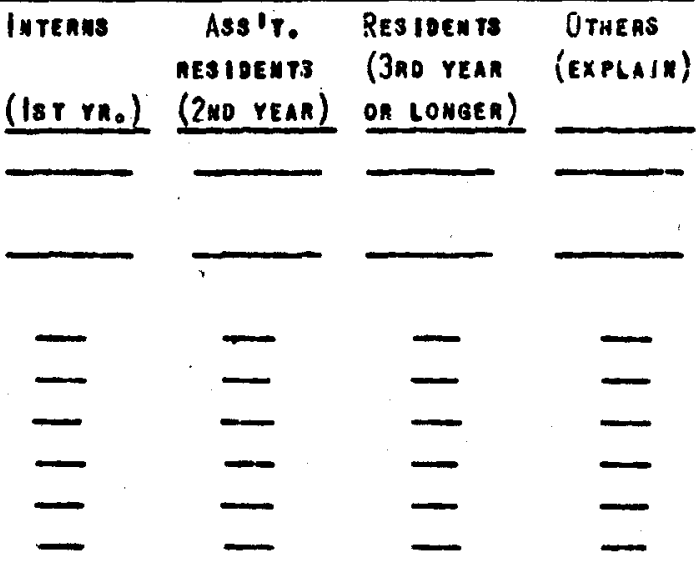

17. TRAINING IN PEOIA.TRICS

Has muRsing supeavison of specified unit had postgraouate tralming in peolatrics:

AT ALL TIMES IE No, STATE nOUNS OA OUTY Yes No

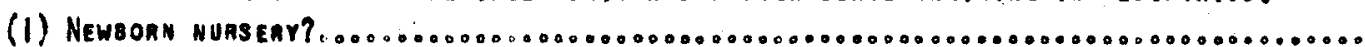

(2) Prematuae unit?.

(3) Peolathic unit? 
18. OUT-PATIENT DEPARTHENT

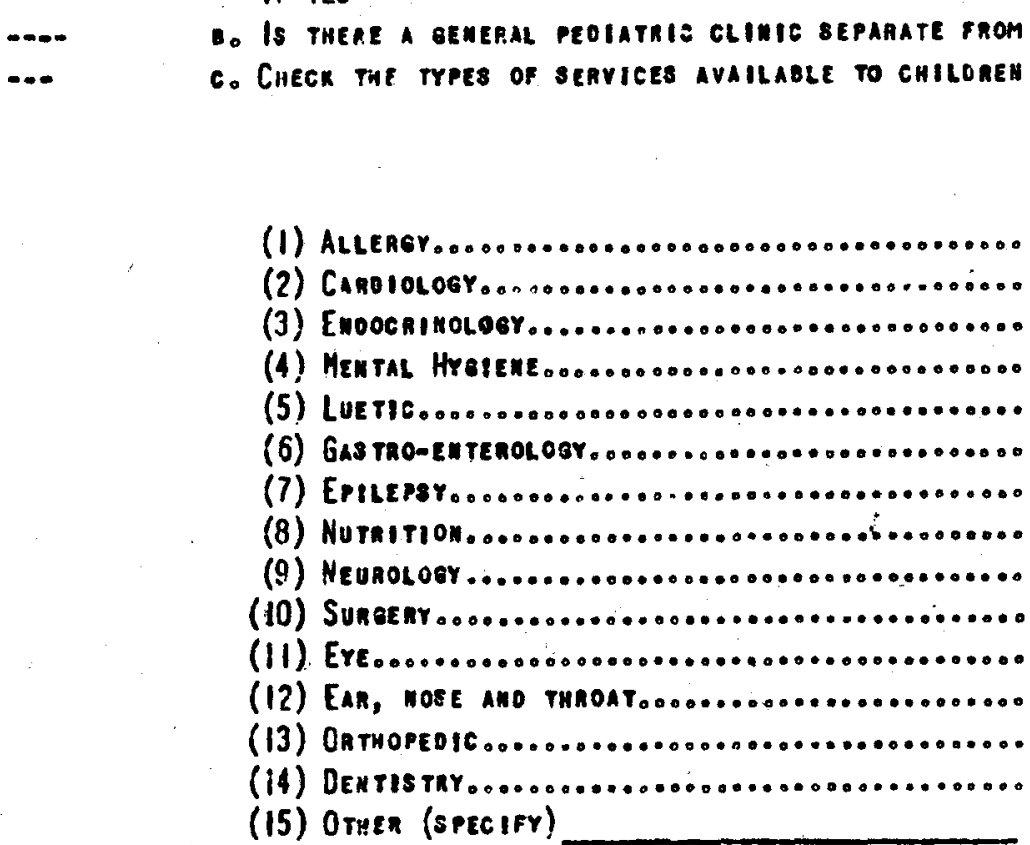

\begin{tabular}{|c|c|c|c|}
\hline GIVEN IN & PARATE & Given IX & Nor \\
\hline CHILDREH'S & CLINIC & NOULT CLINIC & GIVEn \\
\hline
\end{tabular}

D. Extent of senvice ourphe one year:

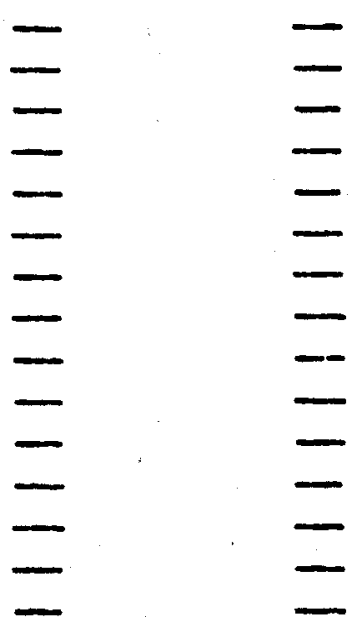

(1) AlL trPEs of service..........

(2) Peoiataics (All chILOREN)

19. OENTAL SERVICE

(i) Extractions

(2) FILLIHES.

(3) Prophrlaxis

(4) Onthooontal

(1) Number of chiloren treateo

(2) TOTAL MUMBER OF TAEATMENTS FOR ALL CMILOREW............................... Remanxs

20.

\section{Remaxe}

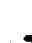

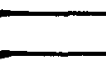

21. In osder that the hospital may 3e classified tae same in both the Hospital Complssion ano peolatric Studies, defore this foloen is separateo faom the Hospital Commission Scheoule, please emten the ITEMS CHECKEO IN THE POLLOWINE TKREE SECTIONS: A-3: A-4: A-6 (ITEMS 1, 4 On 5): 


\section{Schedule 1-D. Supplementary Information on General, \\ Pediatric and Maternity Hospitals with 25 or More Beds}

Date

Code No.

1. NAME AND LOCATION OF HOSPITAL:

Name

Street and number

City or town

County

State

2. ACCOMMODATIONS FOR PATIENTS

a. Give present number of beds actually set up for in-patient care, including children's cribs but excluding bassinets in the nursery -
(1) For all patients
(2) For children only

b. Is there a separate nursery for newborns only?

c. Are there separate wards for infants (other than newborns) ?

d. Are there large wards (10 or more beds) for infants or children

(other than newborn)?

If YES, give number of wards:

(1) 10 to 20 beds

(2) 20 or more beds

$\begin{array}{ll}\text { Yes } \square & \text { No } \\ \text { Yes } \square & \text { No } \\ \text { Yes } \square & \text { No }\end{array}$

Patient Service Data For One Year

Data for year ended 194

(Same year to be used for all annual data requested)

3. NEWBORN (Infants born in this hospital)

a. Number of newborn, including prematures -

Total Nonwhite

(1) Births (excluding stillborn)

(2) Stillborn

(3) Deaths (excluding stillborn)

(4) Newborn (live babies) discharged (1 minus 3)

b. Days of care to newborn

4. AUTOPSIES (Other than coroner's cases)

Number of autopsies -

(1) Stillbirths

(2) Newborn (including prematures)

(3) Infants and older children

(4) Adults

(5) Total (1 thru 4)

5. PREMATURES

a. Number born in this hospital, by birthweight:

(1) $1000 \mathrm{gms}$ or less_...or under 2 lbs. $3 \mathrm{oz}$.

(2) 1001-1500__or 2 lbs. 3 oz. to 3 lbs. 5 oz.

(3) $1501-2000 \ldots$...or 3 lbs. 5 oz. to 4 lbs. 7 oz.

(4) 2001-2500 or 4 lbs. 7 oz. to 5 lbs. 8 oz.

(5) TOTAL (1 thru 4)

b. Number born outside and admitted to hospital

\section{Medical Service}

6. CARE OF CHILD PATIENTS (one-day record)

On the day of visit to the hospital, enter the number of patients on each type of service and the medical staff responsible for their care.

Type of service -

Total Number of children under care of -

(1) Premature

(2) Newborn (excluding prematures)

(3) Medical

(4) Surgical

(5) Orthopedic

(6) Contagious

(7) Other (specify)

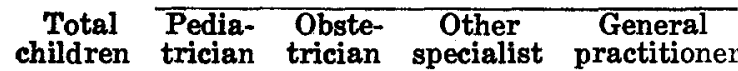


7. CONSULTANT SERVICE FOR CHILDREN

Check type of services for which specialists are available for consultation and service for children.

Diplomates* Non-diplomates
(1) Eye
(6) Neurological surgery
(2) Ear, nose \& throat ..
(7) Psychiatry \& neurology .
(3) Surgery
(8) Dermatology
(4) Orthopedic surgery
(9) Allergy
(5) Plastic surgery
(10) Cardiology
*Diplomates are holders of certificates from Amer. Exam. Boards of Med. Spec.

Diplomates* Non-diplomates

\section{FOLLOW-UP SERVICE}

Is there a follow-up service that insures that newborn infants are discharged to the care of a private physician or a well-baby clinic for:

a. Prematures

b. Other newborn infants

9. TYPE OF STAFF

Are all doctors in the community allowed to care for child patients in this hospital?

If NO, are exclusions based on (check one or more) -
a. Professional qualifications of physician
d. Use as teaching hospital
b. Race of physician
e. Private ownership of hospital
c. Bed capacity of hospital
f. Other (specify)

\section{Nursing Service}

10. NURSING SERVICE FOR INFANTS AND CHILDREN (one-day record)

For the day prior to the visit to the hospital, record the number of infants and children cared for and the total nursing hours.

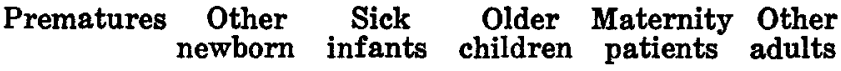

a. Total number of patients

b. Total hours of nursing service -

(1) Student and graduate nurses -

(a) 7 a.m. to 3 p. m.

(b) $3 \mathrm{p} . \mathrm{m}$. to $11 \mathrm{p} . \mathrm{m}$.

(c) 11 p. m. to 7 a. m.

(2) Other attendants -

(a) 7 a. m. to 3 p. m.

(b) $3 \mathrm{p} . \mathrm{m}$. to $11 \mathrm{p} . \mathrm{m}$.

(c) $11 \mathrm{p} . \mathrm{m}$. to 7 a. m.

11. NURSING TECHNIQUES

a. Check whether gowns and masks are worn by doctors and nurses -

(1) Caring for prematures

(2) Caring for other newborn (well)

(3) Caring for infants other than newborn

b. Are infants held while being fed?

Yes $\square \quad$ No $\square$

12. MISCELLANEOUS EQUIPMENT

a. Are the following provided individually for each patient -

$\frac{\text { Infants }}{\text { Yes No }} \frac{\text { Older children }}{\text { Yes No }}$

(1) Thermometer?

(2) Towel?

(3) Bedpan?

(4) Washbasin?

b. Is there running water for hand washing in the nursery?

Yes

No $\square$

13. EXAMINATION OF EMPLOYEES

a. Are chest $x$-rays required for all nurses and attendants?

Yes $\square \quad$ No 
14. MILK AND MILK PREPARATION

\section{Feeding of Infants and Older Children}

a. Is there a room used exclusively for preparation of formulae?

If YES, is it in a part of the hospital completely separate from patient area?

b. Are stock formulae used - (1) For the newborn?

(2) For other infants?

e. Are all milk mixtures sterilized - (1) For the newborn?

(2) For other infants?

d. Are formulae cultured periodically?

If YES, state frequency

e. Check type of milk used most commonly in preparing feedings -

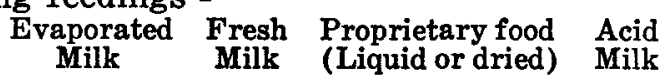

(1) For newborn

(check one)

(2) For other infants -........ (check one)

f. Method of preparing milk mixtures (check one)

$\square$ (1) Aseptic mixing of previously sterilized ingredients.

(2) Mixing of ingredients, pouring into nursing bottles, and terminal sterilization.

$\frac{\text { For infants }}{\text { Yes No }} \frac{\text { For older children }}{\text { Yes No }}$

g. Is all milk pasteurized?

h. Is any bulk milk used?

15. VITAMIN SUPPLEMENTS

a. Is supplementary Vitamin $\mathrm{C}$ given routinely?

b. Is supplementary Vitamin $D$ given routinely?

16. INCIDENCE OF BREAST FEEDING IN NEWBORN NURSERY (one-week record)

The following data are desired for all newborns discharged alive during the past week.

Type of feeding at time of discharge -

\begin{tabular}{|c|c|}
\hline Number discharged & Age at day of discharge \\
\hline $\begin{array}{l}\text { alive during } \\
\text { past week }\end{array}$ & $\begin{array}{l}\text { Less than } \\
8 \text { days }\end{array}$ \\
\hline
\end{tabular}

(1) Breast only

(2) Breast and bottle

(3) Bottle only

(4) Unknown

(5) Total (1 thru 4)

\section{Laboratory Department}

17. LABORATORY EXAMINATIONS

a. Are the following types of routine laboratory examinations made on all children admitted for more than 24 hours?
(1) Red count
Yes No
Yes No
(2) White count
(5) Urinalysis
(3) Differential
(6) Serologic test for syphilis...-
(7) Tuberculin test

(4) Hemoglobin

b. Check whether the following types of laboratory services are available, and give the number of such services performed for children hospitalized during one year.

$$
\frac{\text { Available Estimate No. }}{\text { Yes No of services }}
$$

(1) Blood level for sulfas

(2) Typing pneumococci

(3) Typing of $\mathrm{Rh}$

(4) Sedimentation rate

(5) Fragility of red cells

(6) Blood $\mathrm{CO}_{2}$
(7) Blood sugar

(8) Blood cultures

(9) Serum protein

(10) Serum Ph.

(11) Serum chlorides

(12) Serum calcium
Available Estimate No. Yes No of services

18. BLOOD SERVICE

a. Is there a blood or plasma bank in the hospital?

Yes $\square$ No Yes $\square$ No

b. Number of transfusions during one year to children -
(1) Under 1 month of age
(2) 1 month to 1 year
(3) 1 year or older

c. Is $\mathrm{Rh}$ negative blood readily available?

Yes $\square \quad$ No

19. INTRAVENOUS THERAPY

a. Estimate number of $\mathrm{I}$. V. injections of fluid given to infants and children during 1 yr. 


\section{Miscellaneous}

20. LAUNDRY FOR NURSERY

a. Is the laundry for the nursery handled separately from that of the rest of the hospital?

b. Are the clothing and linen for the nursery sterilized?

21. DIETARY DEPARTMENT

a. Are operations of dietary department checked by health department? ...... Yes $\square$ No $\square$

b. Total number of meals served during past 7 days

c. Refrigeration space: cu. ft.

d. Kitchen space: sq. ft.

e. Glassware: a. Method of washing-(1) By hand $\square \quad$ (2) By mechanical dishwasher $\square$ b. Detergent used - (1) Soap

f. Are utensils (including dishes) dried with towels?

g. Has there been a special training course for kitchen employees in the sanitation

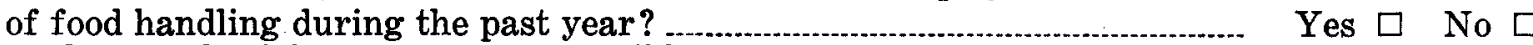

$h$. Is there a physician.or nurse responsible for making periodic examinations of kitchen help for communicable disease? Yes $\square$ No $\square$; Frequency of examination

\section{Incident of Disease During One Year}

22. ACUTE POLIOMYELITIS IN INFANTS AND CHILDREN

a Do you admit acute cases (1) For hospital care?

(2) For diagnosis and emergency treatment only?

If YES for (1) or (2):

b. Number of acute cases admitted during one year

c. Total patient days of service rendered these cases

d. Check where acute cases are cared for (check one) :
$\square$ (1) In separate contagious disease ward
(2) In separate rooms in pediatric ward
(3) In general pediatric wards
$\square$ (4) Elsewhere in hospital

e. Check who is responsible for care of acute poliomyelitis cases:
(1) Pediatrician
(2) Orthopedic surgeon
(3) Other (specify)

23. RHEUMATIC FEVER AND/OR RHEUMATIC HEART DISEASE

a. Number of cases admitted during one year

b. Total patient days of service rendered these cases

c. Care of patients:

(1) Is the pediatric staff responsible for care of patients?

(2) Are patients seen by cardiac and/or rheumatic fever consultant?

(3) Is electrocardiography available?

(4) Is fluoroscopy or x-ray available?

(5) Are facilities for sedimentation rate available?

d. Disposition of patients:

(1) Is convalescent institution or home available?

(2) Is discharge of patients delayed because of inadequate facilities for convalescent care?

(3) Are patients usually discharged home?

24. OTHER SPECIFIC DISEASES DURING ONE YEAR

a. Number of cases in newborn nursery of :
(1) Diarrhea
(2) Epidemic diarrhea
(3) Impetigo

b. Number of cases of gonococcus vaginitis developing in infants and children after admission

c. Number of cases of cross-infection to children developing from primary communicable disease cases admitted to hospital:
(1) Scarlet fever
(3) Measles
(2) Whooping cough
(4) Diphtheria
(5) Mumps
(6). Chicken pox

d. Number of deaths from epidemic diarrhea in newborn nursery

Name of pediatrician

Return this schedule to: 
ACADEMY OF PEDIATRICS STUDY OF CHILD HEALTH SERVICES

Schedule I-E. Nervous and Mental Hospitals, Including Institutions for the Feeble-Minded

Date

Code No.

1. NAME AND LOCATION OF HOSPITAL

Name

Street and number

City or town

County

State

2. TYPE OF OWNERSHIP OR CONTROL (check one) Governmental

(1) Federal

(2) State

(3) City, town, Village or other municipality

(4) County

(5) City-County

Non-profit
(6) Church
(7) Non-profit
association

Non-Governmental

(11) Other (explain)

3. ACCREDITATIONS AND APPROVALS (check)

a. American Medical Association Registry: Yes $\square$

b. American College of Surgeons: (1) Full approval $\square$

(2) Provisional approval $\square \quad$ (3) Not approved

4. MEDICAL SCHOOL AFFILIATION

a. Is the hospital or institution affiliated with a medical school?

If $Y E S$, check purpose: $\quad$ Yes No

Yes $\square \quad$ No $\square$

(1) Instruction undergraduate students

(2) Postgraduate training

(3) Research and investigation

5. ADMISSION OF CHILDREN

a. Are children admitted?

If NO, no further items on this schedule are to be filled.

Yes $\square \quad$ No $\square$

If $Y E S$,

b. Are children admitted from outside the State?

Yes $\square \quad$ No

c. Are children admitted from-

(1) Entire State $\square$ (2) Section of State only $\square$ or

(3) County or local community only

d. Check whether services for children are available to:

(1) White only $\square \quad$ (2) White and nonwhite $\square$ or (3) Nonwhite only $\square$

e. Is service restricted by policy or regulation as to length of residence? -.-If $Y E S$, explain

f. Is service restricted by policy or regulation as to economic status? If $Y E S$, explain

g. Age: (1) Earliest age for admission

(2) Classified as a child until

(3) No longer cared for after age.

Yes $\square \quad$ No

Yes

No $\square$

6. ACCOMMODATIONS FOR CHILDREN

a. Give present number of beds actually set up for in-patient care, including children's cribs but excluding bassinets in newborn nursery-

(1) For all patients

(2) For children only

b. Are children entirely separated from adult patients (that is, are permanent

Yes No facilities set aside for exclusive use of children?)

If $N O$, what provisions are there for children?

c. Are infants separated from older children?

d. Is there separation of nonwhite from white children?

e. Is there separation by degree of mental defectiveness?

f. Is there separation by behavior difficulty?

g. Are there large wards (10 or more beds) for infants or children?

If $Y E S$, give number of wards (1) 10 to 20 beds

(2) 20 or more beds

years

years

years 
7. PATIENT SERVICE DATA FOR ONE YEAR

Data for year ended 194

(Same year to be used for all annual data requested)

a. Summary of services rendered Number of patients :

(1) Remaining in hospital beginning of year

(2) Admitted (exclude births)

(3) Total treated (1 and 2)

(4) Discharged (except deaths)

(5) Died (exclude stillbirths)

(6) Total discharged and died (4 and 5)

(7) Remaining end of year (3 minus 6 )

b. Total patient days of service rendered:
(1) All patients
(2) All children
(3) Nonwhite children

c. Average cost per patient-day (i.e. per capita in-patient expense)

All
Patients

d. Number of children on waiting list for admission at present time

8. TYPES OF CHILDREN'S CASES ADMITTED

a. Check types of children's cases admitted and for what purpose

Type of case :

(1) Mental deficiency

(2) Mongolism

(3) Psychoses

(4) Psychoneuroses and neurotic traits

(5) Convulsive disorders

(6) Psychopathic personality

(7) Neurological disorders without mental deficiency

(8) Cerebral palsy

(9) Other (specify)

\begin{tabular}{|c|c|c|c|c|}
\hline \multirow{2}{*}{\multicolumn{2}{|c|}{ Admitted }} & \multicolumn{3}{|r|}{ ther for- } \\
\hline & & Study & Treat- & Custodia \\
\hline$\overline{\text { Yes }}$ & No & & ment & care \\
\hline$\square$ & $\square$ & $\square$ & $\square$ & $\square$ \\
\hline$\square$ & $\square$ & $\square$ & $\square$ & $\square$ \\
\hline$\square$ & $\square$ & $\square$ & $\square$ & $\square$ \\
\hline$\square$ & $\square$ & $\square$ & $\square$ & $\square$ \\
\hline$\square$ & $\square$ & $\square$ & $\square$ & $\square$ \\
\hline$\square$ & $\square$ & $\square$ & $\square$ & $\square$ \\
\hline$\square$ & $\square$ & $\square$ & $\square$ & $\square$ \\
\hline$\square$ & $\square$ & $\square$ & $\square$ & $\square$ \\
\hline$\square$ & $\square$ & $\square$ & $\square$ & $\square$ \\
\hline$\square$ & $\square$ & $\square$ & $\square$ & $\square$ \\
\hline
\end{tabular}

9. FOSTER-HOME CARE

a. Does the hospital use foster homes for the care of children?

Yes $\square \quad$ No

b. If $Y E S$, number of children so placed in year of report

10. CARE OF SICK CHILDREN

a. Is there space allocated in hospital for care of children with acute illness? Yes $\square$ No $\square$

(1) If $Y E S$, total number of beds set aside for sick children

(2) If NO, are sick children sent to another hospital?

Yes $\square \quad$ No $\square$

b. Are children who develop communicable disease in the hospital (check one) :

$\square$ (1) Kept on ward?

(2) Isolated in infirmary?

(3) Sent to communicable disease hospital?

c. Is there a routine isolation procedure for children on admission?

If YES, check whether for: (1) 1 day only $\square \quad$ (2) 2-7 days only

Yes $\square \quad$ No $\square$

(3) 8 days or longer

11. OUT-PATIENT DEPARTMENT

a. Is there an out-patient department?

If $Y E S$ :

b. Is there a children's clinic separate from clinics for aduits?

c. Extent of service during one year:

Patients Visits

Yes $\square \quad$ No

Yes $\square \quad$ No $\square$

(1) All types of service

(2) Services for children 
12. LABORATORY FACILITIES

a. Check whether facilities for the following procedures are-

(1) Blood counts

(2) Urinalyses

(3) Electroencephalography

(4) X-ray and/or fluoroscopy

13. ADMINISTRATOR

a. Training (check one) :
$\square$ (1) Medical doctor
(2) Clergyman
(3) Osteopath
(4) Chiropractor
(5) Reg. or grad. nurse: Nun, sister, mother
$\square$ (6) Reg. or grad. nurse: Not nun, sister, mother
$\square$ (7) Nun, sister, mother: Not reg. or grad. nurse
$\square$ (8) Other (specify)

14. MEDICAL DIRECTOR OR CHIEF

a. Is there a medical director in addition to the administrator $\square$ or are they the same? $\square$

b. Work status: (1) Full-time, paid $\square$ (2) Part-time, paid $\square$ (3) Unpaid $\square$

c. Medical specialty (check one) :
$\square$ (1) Psychiatry
$\square$ (3) Other

(2) Pediatrics

(4) None

d. Diplomate (certified by Am. Exam. Boards of Med. Spec.)

e. Hospital training in neuropsychiatry after graduation from medical school

Yes $\square \quad$ No $\square$ mos.

15. NUMBER OF STAFF MEMBERS

a. General medical staff (incl. consultants)
(1) Psychiatrists
(2) Pediatricians
(3) General practitioners
(4) Internists
(5) Otolaryngologists
(6) Surgeons
(7) Neurological surgeons
(8) Neurologists
(9) Other (specify)

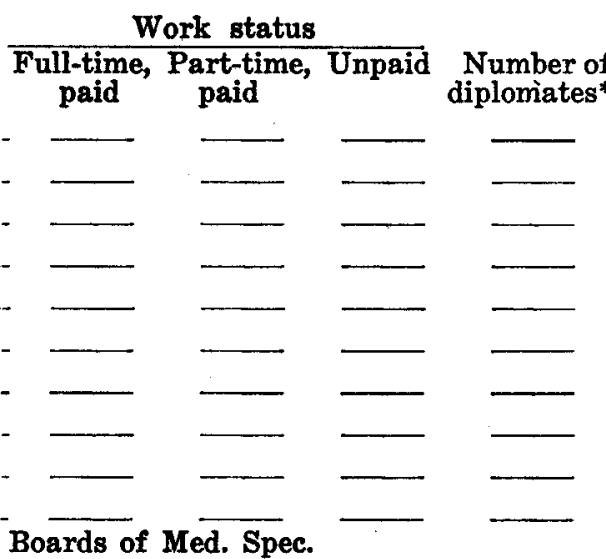

*Diplomates are holders of certificates from Am. Exam. Boards of Med. Spec.

b. Medical house staff :

(1) Interns (1st year)

(2) Ass't residents (2nd year)

(3) Residents (3rd year or longer)

(4) Other (explain)

c. Nursing staff :

(1) Graduate nurses

(2) Student nurses

(3) Custodians*

*Include practical nurses, attendants, nurses' aides, maids and orderlies.

d. Auxiliary staff :

(1) School teachers

(2) Occupational therapists

(3) Psychologists

(4) Medical social workers

(5) Psychiatric social workers

(6) Physiotherapists

(7) Other (explain)
Total Number assigned number to children only

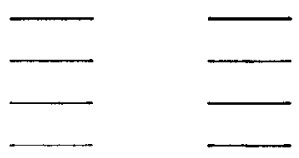

Total Number assigned number to children only

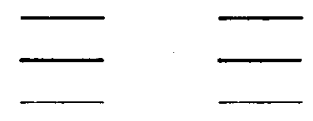

Work status of number assigned to children only Full-time, Part-time, Unpaid Work status of all Full-time, Part-time, Unpaid paid paid 


\section{INSTRUCTIONS TO THE FIELD AGENT FOR FILLING SCHEDULE. I-E}

An entry must be made for each item. If there are items for which the hospital is not able to supply data, make a note to that effect through or by the item. All items requiring a "yes"-"no" answer are easily filled out. Where figures are asked for, the hospital staff may require a little time to get the data from their records and mail them to you; leave a stamped self-addressed envelope for this purpose.

Most of the items are self-explanatory. A few detailed instructions dealing with certain more complex items are given below for your assistance.

Data for one year................

Nonwhite

2 Type of ownership.

5a Admission of children.

$5 \mathrm{e}$ Length of residence......

$5 f$ Economic status

$5 g$ (3) Age.

6f Behavior

6g Large wards

7 Patient service data....

7c Cost per patient-day....

9 Foster-home care.

12 Laboratory facilities

14 Medical director.

15 Staff members.

15a General medical staff..
Ask your informant to select the last year for which the hospital has complete data. Use this same year for answering all questions that deal with data on an annual basis (items $7 \mathrm{a}-\mathrm{c}, 9 \mathrm{~b}$, and 11c).

Include Negro and other nonwhite races.

Check one item only. All church operated or affiliated institutions check (6). Include non-profit corporation under (7).

Fill one copy of this schedule items 1-4 for each nervous and mental hospital or institution, but only for those admitting children should the schedule from item $5 \mathrm{~b}$ on be filled.

Explain if patient has to be resident of State or community for any period of time prior to admission.

Explain if patient has to declare income level or inability to pay for service.

Specify if there is an upper age limit after which patients are transferred to another institution or discharged; otherwise, if the patients are kept until death, enter "death".

If 'disturbed' patients are separated from 'quiescent' patients regardless of type of case, check "yes".

If there are wards of 10 or more beds, state number of wards (not number of beds on these large wards).

The answers to all parts of this question should be entered carefully. These items are fundamental data used as the basis for other calculations.

Patient-day expense for in-patients is desired here. It is obtained by dividing total operating expenses for in-patients by total number of patient days reported in item $7 \mathrm{~b}(1)$ (all patiënts).

This includes placing out in private homes of children who have been residents in the institution and/or those coming to the out-patient department.

If available in this hospital, make no entries for "readily available elsewhere".

Even if the administrator and the medical director are the same person, fill items 13 and 14.

In institutions employing the services of religious orders, entries should be made as though the positions indicated were filled by full-time or part-time paid workers, whether or not they received remuneration.

A full-time employee is one who works full time in the hospital even though he devotes part of his time to several jobs within the hospital during different periods of the day or night. A part-time employee is one who does not work full time for the hospital.

Be sure to include under the proper classification the medical director and the administrator if he is a physician.

After the schedule is complete, review it thoroughly for omissions and inconsistencies. Sign your name below in order that any questions that arise at a later date may be referred to you, and return the schedule promptly to the State office. Leave a duplicate completed copy at the hospital for their files, if they desire.

Name of field agent:

Return this schedule to: 


\section{ACADEMY OF PEDIATRICS STUDY OF CHILD HEALTH SERVICES}

Schedule I-F. Tuberculosis Hospitals; Sanatoriums and Preventoriums Admitting

Tuberculous Patients

Date

Code No.

1. NAME AND LOCATION OF HOSPITAL

Name

Street and number

City or town __ County State

2. TYPE OF OWNERSHIP OR CONTROL (check one)
(1) Federal Governmental
(2) State
(3) City, town, village or other municipality
(4) County
(5) City-County

Non-profit

(6) Church

(7) Non-profit association

Non-Governmental
Proprietary

$\square$ (8) Individual

$\square$ (9) Partnership

$\square$ (10) Corporation

(11) Other (explain)

3. ACCREDITATIONS AND APPROVALS (check)

a. American Medical Association Registry: Yes $\square$ No

b. American College of Surgenns: (1) Full approval
(2) Provisional approval
(3) Not approved

4. MEDICAL SCHOOL AFFILIATION

a. Is the hospital affiliated with a medical school?

If $Y E S$, check purpose:

(1) Instruction undergraduate students

(2) Postgraduate training

(3) Research and investigation

5. ADMISSION OF CHILDREN

a. Are children admitted?

If NO, no further items on this schedule $\underset{*}{\operatorname{are}}$ to be filled.

\section{Yes No}

Yes

$\square \quad$ No

If $Y E S$,

b. Are children admitted from outside the State?

c. Are children admitted from -
(1) Entire State
(2) Section of State only

(3) County or local community only

d. Check whether services for children are available to:

(1) White only $\square \quad$ (2) White and nonwhite $\square$ or (3) Nonwhite only

e. Is service restricted by policy or regulation as to length of residence?

If $Y E S$, explain

f. Is service restricted by policy or regulation as to economic status?

If $Y E S$, explain

g. Earliest age for admission

Yes $\square \quad$ No $\square$

Yes $\square \quad$ No $\square$
Yes $\square \quad$ No $\square$

Yes $\square \quad$ No $\square$ years

6. ACCOMMODATIONS FOR CHILDREN

a. Give present number of beds actually set up for in-patient care, including

children's cribs but excluding bassinets in newborn nursery -

(1) For all patients

(2) For children only

b. Are children entirely separated from adult patients (that is, are permanent Yes No

facilities set aside for exclusive use of children?)

If $N O$, what provisions are there for children?

c. Are infants separated from older children?

d. Is there separation of nonwhite from white children?

e. Is there separation by stage of disease?

f. Are there large wards (10 or more beds) for infants or children?

If $Y E S$, give number of wards (1) 10 to 20 beds

(2) 20 or more beds 
7. PATIENT SERVICE DATA FOR ONE YEAR

Data for year ended 194

(Same year to be used for all annual data requested.)

a. Summary of services rendered Number of patients:

(1) Remaining in hospital beginning of year

(2) Admitted (exclude births)

(3) Total treated (1 and 2)

(4) Discharged (except deaths)

(5) Died (exclude stillbirths)

(6) Total discharged and died (4 and 5)

(7) Remaining end of year (3 minus 6)

* Specify upper age limit of patients classed as children

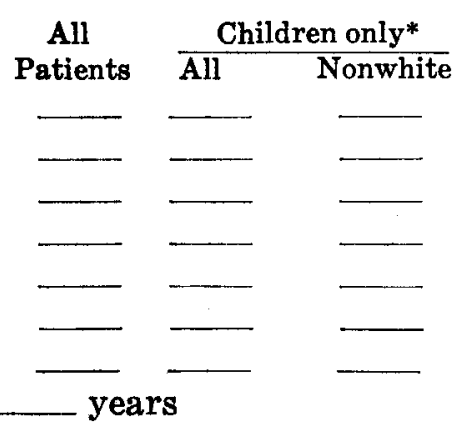

b. Total patient days of service rendered:

(1) All patients $\longrightarrow$, (2) All children $\longrightarrow$, (3) Nonwhite children

c. Average cost per patient-day (i.e. per capita in-patient expense)

d. Percent of children usually "bed patients"

e. Number of children on waiting list for admission at present time

$\$$

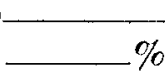

8. TYPES OF CHILDREN'S CASES ADMITTED

a. Check types of children's cases admitted and for what purpose.

Type of case:

(1) Pulmonary tuberculosis, 1st infection, active

(2) Pulmonary tuberculosis, 1st infection, other

(3) Pulmonary tuberculosis, reinfection, active

(4) Pulmonary tuberculosis, reinfection, other

(5) Bone and joint tuberculosis

(6) Other tuberculosis

(7) Non-tuberculosis contacts only

9. FOSTER-HOME CARE

a. Does the hospital use foster homes for the care of children?

Admitted

If admitted, check whether for-

b. Number of children so placed in year of report

10. CARE OF SICK CHILDREN

a. Is there space allocated in hospital for care of children with acute illness? Yes $\square$ No $\square$

(1) If $Y E S$, total number of beds set aside for sick children

(2) If NO, are sick children sent to another hospital?

Yes $\square$ No $\square$

b. Are children who develop communicable disease in the hospital (check one) :

(1) Kept on ward?

(2) Isolated in infirmary?

(3) Sent to communicable disease hospital?

c. Is there a routine isolation procedure for children on admission?

If YES, check whether for: (1) 1 day only $\square \quad$ (2) 2-7 days only Yes $\square \quad$ No

(3) 8 days or longer

11. OUT-PATIENT DEPARTMENT

a. Is there an out-patient department?

Yes $\square \quad$ No

If $Y E S$ :

b. Is there a children's clinic separate from clinics for adults?

c. Extent of service during one year:

Patients Visits

Yes $\square \quad$ No
(1) All types of service
(2) Services for children

12. LABORATORY FACILITIES

a. Check whether facilities for the following procedures are -

(1) Blood counts

(2) Sedimentation rates

(3) Urinalyses

(4) Sputum smears

(5) Guinea pig inoculations

(6) X-ray

(7) Fluoroscopy

$\begin{array}{cccc}\begin{array}{c}\text { In this } \\ \text { hospital }\end{array} & \begin{array}{c}\text { Readily available } \\ \text { elsewhere }\end{array} \\ { } \text { Yo } } & \text { Yes } & \text { No } \\ \square & \square & \square & \square \\ \square & \square & \square & \square \\ \square & \square & \square & \square \\ \square & \square & \square & \square \\ \square & \square & \square & \square \\ \square & \square & \square & \square \\ \square & \square & \square & \square\end{array}$


13. ADMINISTRATOR
a. Training (check one) :
$\square$ (1) Medical doctor
(5) Reg. or grad. nurse: Nun, sister, mother
(2) Clergyman
(6) Reg. or grad. nurse: Not nun, sister, mother
(3) Osteopath
(7) Nun, sister, mother: Not reg. or grad. nurse
(4) Chiropractor
(8) Other (specify)

\section{MEDICAL DIRECTOR OR CHIEF}

a. Is there a medical director in addition to the administrator $\square$ or are they the same?

b. Work status:

(1) Full-time, paid $\square$

(2) Part-time, paid

(3) Unpaid $\square$

c. Medical specialty: (check one)
(1) Internal medicine
(3) Other (specify)
(2) Pediatrics
(4) None

d. Diplomate (certified by Am. Exam. Boards of Spec.) Yes $\square \quad$ No $\square$

e. Hospital training in tuberculosis after graduation from medical school mos.

15. NUMBER OF STAFF MEMBERS

(1) Pediatricians

(2) General practitioners

(3) Internists

(4) Chest specialists

(5) Surgeons

(6) Psychiatrists

(7) Radiologists

(8) Neurologists

(9) Orthopedic surgeons

(10) Otolaryngologists

(11) Other (specify) a. General medical staff (incl. consultants)

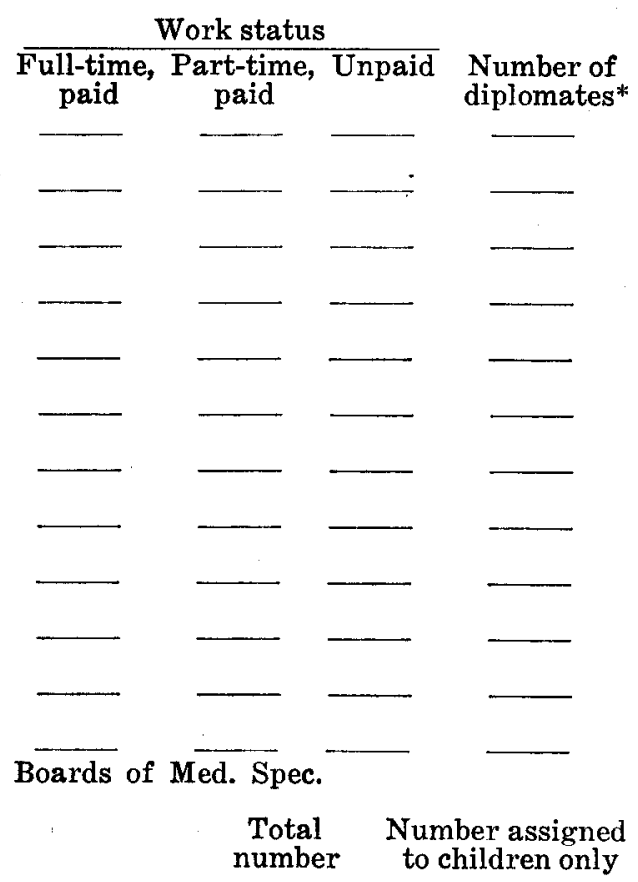

b. Medical house staff :

(1) Interns (1st year)

(2) Ass't residents (2nd year)

(3) Residents (3rd year or longer)

(4) Other (explain)

c. Nursing staff:

(1) Graduate nurses

(2) Student nurses

(3) Custodians*

*Include practical nurses, attendants, nurses' aides, maids and orderlies.

d. Auxiliary staff :

Work status of all

Work status of number assigned to children only Full-time, Part-time, Unpaid $\overline{\text { Full-time, Part-time, Unpaid }}$

(1) School teachers

(2) Occupational therapists

(3) Social workers

(4) Other (explain) 


\section{INSTRUCTIONS TO THE FIELD AGENT FOR FILLING SCHEDULE I-F}

An entry must be made for each item. If there are items for which the hospital is not able to supply data, make a note to that effect through or by the item. . All items requiring a "yes" - "no" answer are easily filled out. Where figures are asked for, the hospital staff may require a little time to get the data from their records and mail them to you; leave a stamped self-addressed envelope for this purpose.

Most of the items are self-explanatory. A few detailed instructions dealing with certain more complex items are given below for your assistance.

Data for one year.

Nonwhite.

2 Type of ownership.

5a Admission of children...

5e Length of residence.......

5f Economic status.

$6 f$ Large wards.

7 Patient service data.....

7c Cost per patient-day....

8 Type of cases.

9 Foster-home care

12 Laboratory facilities

14 Medical director.

15 Staff members

15a General medical staff.
Ask your informant to select the last year for which the hospital has complete data. Use this same year, for answering all questions that deal with data on an annual basis (items $7 a-c, 9 b$, and $11 c$ ).

Include Negro and other nonwhite races.

Check one item only. All church operated or affiliated institutions check (6). Include non-profit corporation under (7).

Fill one copy of this schedule items 1-4 for each tuberculosis hospital or nursing home, but only for those admitting children should the schedule from item $5 b$ on be filled.

Explain if patient has to be resident of State or community for any period of time prior to admission.

Explain if patient has to declare income level or inability to pay for service.

If there are wards of 10 or more beds, state number of wards (not number of beds on these large wards).

The answers to all parts of this question should be entered carefully. These items are fundamental data used as the basis for other calculations.

Patient-day expense for in-patients is desired here. It is obtained by dividing total operating expenses for in-patients by total number of patient days reported in item $7 \mathrm{~b}(1)$ (all patients).

First infection refers to childhood type; reinfection to adult type.

This includes placing out in private homes of children who have been residents in the institution and/or those coming to the out-patient department.

If available in this hospital, make no entries for "readily available elsewhere".

Even if the administrator and the medical director are the same person, fill items 13 and 14.

In institutions employing the services of religious orders, entries should be made as though the positions indicated were filled by full-time or part-time paid workers, whether or not they received remuneration.

A full-time employee is one who works full time in the hospital even though he devotes part of his time to several jobs within the hospital during different periods of the day or night. A part-time employee is one who does not work full time for the hospital.

Be sure to include under the proper classification the medical director and the administrator if he is a physician.

After the schedule is complete, review it thoroughly for omissions and inconsistencies. Sign your name below in order that any questions that arise at a later date may be referred to you, and return the schedule promptly to the State office. Leave a duplicate completed copy at the hospital for their files, if they desire.

Name of field agent:

Return this schedule to: 


\section{ACADEMY OF PEDIATRICS STUDY OF CHILD HEALTH SERVICES}

\section{Schedule I-G. Convalescent and Chronic Hospitals, Rest Homes and Nursing Homes}

Date

Code No.

1. NAME AND LOCATION OF HOSPITAL

Name

Street and number

City or town County State

2. TYPE OF OWNERSHIP OR CONTROL (check one)
(1) Federal Governmental
(2) State
(3) City, town, village or other municipality
(4) County
(5) City-County

3. ACCREDITATIONS AND APPROVALS (check)
Non-Governmental Non-profit
(6) Church
(7) Non-profit association
(8) Individual
$\square$ (9) Partnership
(10) Corporation

\section{(11) Other (explain)}

a. American Medical Association Registry: Yes $\square$ No

b. American College of Surgeons :

(1) Full approval $\square$

(2) Provisional approval

(3) Not approved

4. MEDICAL SCHOOL AFFILIATION

a. Is the institution affiliated with a medical school?

If $Y E S$, check purpose:

(1) Instruction undergraduate students

(2) Postgraduate training

(3) Research and investigation

b. Is the institution affiliated with a hospital from which the majority of patients come?

If $Y E S$, give: Name

\section{Address}

Yes $\square \quad$ No $\square$

5. ADMISSION OF CHILDREN

a. Are children admitted?

If $N O$, no further items on this schedule are to be filled.

Yes $\square \quad$ No $\square$

If $Y E S$,

b. Are children admitted from outside the State?

Yes $\square \quad$ No $\square$

c. Are children admitted from -
(1) Entire State
(2) Section of State only
or
(3) County or local community only

d. Check whether services for children are available to:
(1) White only
(2) White and nonwhite $\square$
or (3) Nonwhite only

e. Is service restricted by policy or regulation as to length of residence?

If $Y E S$, explain

f. Is service restricted by policy or regulation as to economic status? .......... If $Y E S$, explain

g. Earliest age for admission

Yes $\square \quad$ No

Yes $\square \quad$ No $\square$ years

6. ACCOMMODATIONS FOR CHILDREN

a. Give present number of beds actually set up for in-patient care, including children's cribs but excluding bassinets in newborn nursery -
(1) For all patients

(2) For children only

b. Are children entirely separated from adult patients (that is, are permanent facilities set aside for exclusive use of children?) If $N O$, what provisions are there for children?

c. Are infants separated from older children?

d. Is there separation of nonwhite from white children?

e. Are there large wards (10 or more beds) for infants or children? If $Y E S$, give number of wards (1) 10 to 20 beds

(2) 20 or more beds 
7. PATIENT SERVICE DATA FOR ONE YEAR

Data for year ended 194_ (Same year to be used for all annual data requested.)

a. Summary of services rendered

Number of patients :

(1) Remaining in hospital beginning of year

(2) Admitted

(3) Total treated (1 and 2)

(4) Discharged (except deaths)

(5) Died

(6) Total discharged and died (4 and 5)

(7) Remaining end of year (3 minus 6).

*Specify upper age limit of patients classed as children:

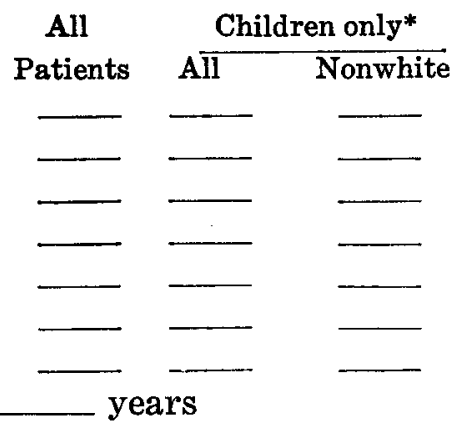

b. Total patient days of service rendered:

(1) All patients $\ldots$, (2) All children _ _, (3) Nonwhite children

c. Average cost per patient-day (i.e. per capita in-patient expense)

d. Percent of children usually "bed patients"

e. Number of children on waiting list for admission at present time

$\$$

TYPES OF CHILDREN'S CASES ADMITTED

a. Check types of children's cases admitted and for what purpose.

Type of case :

(1) Pulmonary tuberculosis

(2) Other types of tuberculosis

(3) Rheumatic fever/rheumatic heart disease

(4) Other medical convalescence

(5) Surgery

(6) Orthopedics

(7) Mental deficiency

(8) Behavior and/or feeding problems

(9) Allergy

(10) Convulsive disorders

(11) Other

9. FOSTER-HOME CARE

a. Does the hospital use foster homes for the care of children?

Yes $\square \quad$ No $\square$

b. Number of children so placed in year of report

10. CARE OF SICK CHILDREN

a. Is there space allocated in hospital for care of children with acute illness? Yes $\square$ No $\square$

(1) If $Y E S$, total number of beds set aside for sick children

(2) If NO, are sick children sent to another hospital? $\begin{array}{ll} & \text { If admitted, check whether for- } \\ \text { Admitted } & \begin{array}{ll}\text { Diagnosis } \\ \text { and study }\end{array}\end{array}$

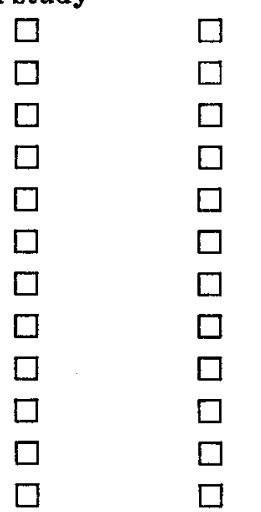

b. Are children who develop communicable disease in the hospital (check one) :

$\square$ (1) Kept on ward?

$\square$ (2) Isolated in infirmary?

$\square$ (3) Sent to communicable disease hospital?

c. Is there a routine isolation procedure for children on admission?

If $Y E S$, check whether for: (1) 1 day only $\square \quad$ (2) 2-7 days only

Yes $\square \quad$ No $\square$

(3) 8 days or longer

11. OUT-PATIENT DEPARTMENT

a. Is there an out-patient department?

If $Y E S$ :

Yes $\square \quad$ No $\square$

b. Is there a children's clinic separate from clinics for adults?

c. Extent of service during one year: Patients Visits

Yes $\square \quad$ No $\square$

(1) All types of service

(2) Services for children 
12. LABORATORY FACILITIES

a. Check whether facilities for the following procedures are -
(1) Blood counts
(2) Sedimentation rates
(3) Urinalyses
(4) Streptococcus cultures
(5) Tuberculin tests
(5) X-ray and/or fluoroscopy

13. ADMINISTRATOR

a. Training (check one) :
$\square$ (1) Medical doctor
$\square$ (2) Clergyman
$\square$ (5) Reg. or grad. nurse: Nun, sister, mother
$\square$ (3) Osteopath
$\square$ (4) Chiropractor
$\square$ (6) Reg. or grad. nurse: Not nun, sister, mother
$\square$ (7) Nun, sister, mother: Not reg. or grad. nurse
$\square$ (8) Other (specify)

14. MEDICAL DIRECTOR OR CHIEF

a. Is there a medical director in addition to the administrator $\square$ or are they the same?

b. Work status: (1) Full-time, paid

(2) Part-time, paid $\square$ (3) Unpaid $\square$

c. Medical specialty: (check one)
(1) Internal medicine
(2) Pediatrics
(3) Other (specify)
(4) None

d. Diplomate (certified by Am. Exam. Boards of Med. Spec.)

15. NUMBER OF STAFF MEMBERS

a. General medical staff (incl. consultants)

(1) Pediatricians

(2) General practitioners

(3) Internists

(4) Cardiologists

(5) Surgeons

(6) Psychiatrists

(7) Radiologists

(8) Neurologists

(9) Orthopedic surgeons

(10) Otolaryngologists

(11) Other (specify)

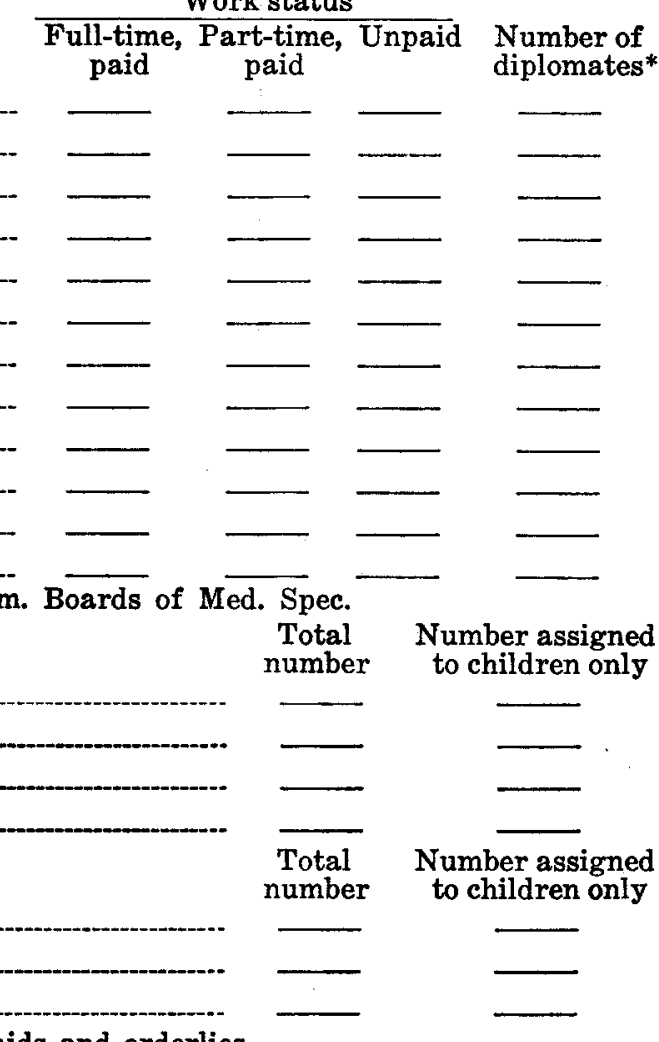

*Diplomates are holders of certificates from Am. Exam. Boards of Med. Spec.

b. Medical house staff:

(1) Interns (1st year)

(2) Ass't residents (2nd year)

(3) Residents (3rd year or longer)

(4) Other (explain)

c. Nursing staff :

(1) Graduate nurses

(2) Student nurses

(3) Custodians*

*Include practical nurses, attendants, nurses' aides, maids and orderlies.

d. Auxiliary staff :

$\frac{\text { Work status of all }}{\text { Full-time, Part-time, Unpaid }} \underset{\text { paid }}{\text { paid }}$

Work status of number assigned to children only Full-time, Part-time, Unpaid paid paid

(1) School teachers

(2) Occupational therapists

(3) Social rorkers

(4) Other (explain) 


\section{INSTRUCTIONS TO THE FIELD AGENT FOR FILLING SCHEDULE I-G}

An entry must be made for each item. If there are items for which the hospital is not able to supply data, make a note to that effect through or by the item. All items requiring a "yes" - "no" answer are easily filled out. Where figures are asked for, the hospital may require a little time to get the data from their records and mail them to you; leave a stamped self-addressed envelope for this purpose.

Most of the items are self-explanatory. A few detailed instructions dealing with certain more complex items are given below for your assistance.

Data for one year

Nonwhite

2 Type of ownership.

5a Admission of children..

5e Length of residence......

$5 f$ Economic status.

6e Large wards

7 Patient service data....

7c Cost per patient-day....

9 Foster-home care.

12 Laboratory facilities.

14 Medical director.

15 Staff members.

15a General medical staff...
Ask your informant to select the last year for which the hospital has complete data. Use this same year for answering all questions that deal with data on an annual basis (items $7 \mathrm{a}-\mathrm{c}, 9 \mathrm{~b}$, and $11 \mathrm{c}$ ).

Include Negro and other nonwhite races.

Check one item only. All church operated or affiliated institutions check (6). Include non-profit corporation under (7).

Fill one copy of this schedule items 1-4 for each convalescent and chronic hospital or nursing home, but only for those admitting children should the schedule from item $5 \mathrm{~b}$ on be filled.

Explain if patient has to be resident of State or community for any period of time prior to admission.

Explain if patient has to declare income level or inability to pay for service.

If there are wards of 10 or more beds, state number of wards (not number of beds on these large wards).

The answers to all parts of this question should be entered carefully. These items are fundamental data used as the basis for other calculations.

Patient-day expense for in-patients is desired here. It is obtained by dividing total operating expenses for in-patients by total number of patient days reported in item $7 \mathrm{~b}$ (1) (all patients).

This includes placing out in private homes of children who have been residents in the institution and/or those coming to the out-patient department.

If available in this hospital, make no entries for "readily available elsewhere".

Even if the administrator and the medical director are the same person, fill iterns 13 and 14.

In institutions employing the services of religious orders, entries should be maide as though the positions indicated were filled by full-time or part-time paid wo rkers, whether or not they received remuneration.

A full-time employee is one who works full time in the hospital even though he devotes part of his time to several jobs within the hospital during different periods of the day or night. A part-tinfe employee is one who does not work full time for the hospital.

Be sure to include under the proper classification the medical director and the ad. ministrator if he is a physician.

After the schedule is complete, review it thoroughly for omissions and inconsistencies. Sign your name below in order that any questions that arise at a later date may be referred to you, and return the schedule promptly to the State office. Leave a duplicate completed copy at the hospital for their files, if they desire.

Name of field agent:

Return this schedule to: 


\section{ACADEMY OF PEDIATRICS STUUDY OF CHILD HEALTH SERVICES}

Schedule I-H. Contagious Disease Hospitals

Date

Code No.

1. NAME AND LOCATION OF HOSPITAL

Name

Street and number

City or town County

State

2. TYPE OF OWNERSHIP OR CONTROL (check one) Governmental

(1) Federal

(2) State

(3) City, town, village or other municipality

(4) County

(5) City-County

Non-Governmental Non-profit

(6) Church

(7) Non-profit association

$\square$ (8) Individual

$\square$ (9) Partnership

$\square$ (10) Corporation

(11) Other (explain)

3. ACCREDITATIONS AND APPROVALS (check)

a. American Medical Association Registry: Yes $\square$ No

b. American College of Surgeons: (1) Full approval
(2) Provisional approval
(3) Not approved

4. MEDICAL SCHOOL AFFILIATION

a. Is the hospital affiliated with a medical school?

If $Y E S$, check purpose:

(1) Instruction undergraduate students

(2) Postgraduate training .

(3) Research and investigation

5. ADMISSION OF CHILDREN

a. Are children admitted?

If $N O$, no further items on this schedule are to be filled.

Yes $\square \quad$ No

If $Y E S$,

b. Are children admitted from outside the State?

Yes $\square \quad$ No

c. Are children admitted from -
(1) Entire State
(2) Section of State only $\square$ or

(3) County or local community only

d. Check whether services for children are available to:

(1) White only $\square \quad$ (2) White and nonwhite $\square$ or (3) Nonwhite only $\square$

e. Is service restricted by policy or regulation as to length of residence? -...-

If $Y E S$, explain

f. Is service restricted by policy or regulation as to economic status? If $Y E S$, explain

g. Earliest age for admission

Yes $\square \quad$ No

Yes $\square \quad$ No $\square$

6. ACCOMMODATIONS FOR CHILDREN

a. Give present number of beds actually set up for in-patient care, including

children's cribs but excluding bassinets in newborn nursery -
(1) For all patients
(2) For children only

b. Are children entirely separated from adult patients (that is, are permanent

facilities set aside for exclusive use of children?)

If $N O$, what provisions are there for children?

c. Are infants separated from older children?

d. Is there separation of nonwhite from white children?

e. Are there large wards (10 or more beds) for infants or children?

If $Y E S$, give number of wards (1) 10 to 20 beds

(2) 20 or more beds

Yes No years 
7. PATIENT SERVICE DATA FOR ONE YEAR

Data for year ended 194

a. Summary of services rendered Number of patients:

(1) Remaining in hospital beginning of year

(2) Admitted (exclude births)

(3) Tótal treated (1 and 2)

(4) Discharged (except deaths)

(5) Died (exclude stillbirths)

(6) Total discharged and died (4 and 5)

(7) Remaining end of year (3 minus 6)

* Specify upper age limit of patients classed as children :

(Same year to be used for all annual data requested.)

b. Total patient days of service rendered:
(1) All patients
(2) All children
(3) Nonwhite children

c. Average cost per patient-day (i.e. per capita in-patient expense)

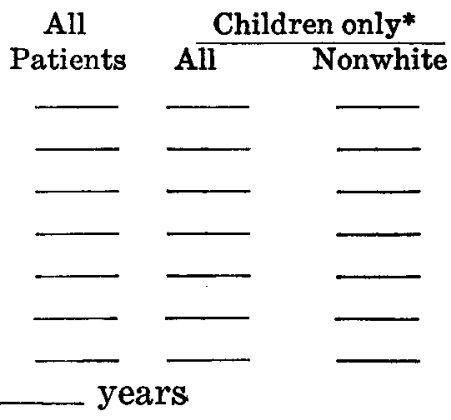

8. TYPES OF CHILDREN'S CASES ADMITTED

a. Check types of children's cases admitted and for what purpose

Type of case:

(1) Poliomyelitis

(2) Meningitis

(3) Encephalitis

(4) Diphtheria

(5) Measles

(6) Mumps

(7) Whooping cough

(8) Chickenpox

(9) Scarlet fever

(10) Typhoid - dysentery

(11) Gonorrhea

(12) Syphilis

(13) Smallpox

(14) Other (specify)

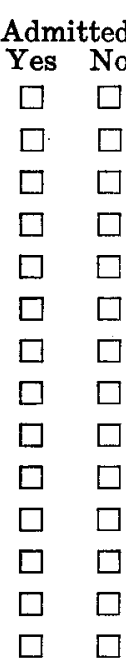

If admitted, check whether forDiag- Treat- Complication nosis ment treat. only

9. CROSS-INFECTION

a. Number of cases of cross-infection to children developing from primary communicable disease cases admitted to hospital during one year:
(1) Scarlet fever
(3) Measles
(5) Mumps
(2) Whooping cough
(4) Diphtheria
(6) Chickenpox

10. EQUIPMENT AND SERVICE

Yes No

a. Is there an operating room in the hospital?

b. Is X-ray service available?

c. Are there facilities for humidifying rooms other than croup kettle?

d. Is there a blood or plasma bank in the hospital?

If $N O$, does hospital have access to blood or plasma bank?

e. Is equipment available for hot packs for polio?

f. Are tracheotomy sets readily available?

g. Are intubation sets readily available?

11. LABORATORY FACILITIES

a. Check whether facilities for the following procedures are -

(1) Blood counts

(2) Sedimentation rates

(3) Urinalyses

(4) Blood cultures

(5) Throat cultures

(6) Stool cultures

(7) X-ray and/or fluoroscopy

In this Readily available

hospital elsewhere

Yes No Yes No 
12. ADMINISTRATOR

a. Training (check one) :
$\square$ (1) Medical doctor
(5) Reg. or grad. nurse: Nun, sister, mother
(2) Clergyman
(6) Reg. or grad. nurse: Not nun, sister, mother
$\square$ (3) Osteopath
(7) Nun, sister, mother: Not reg. or grad. nurse
$\square$ (4) Chiropractor
(8) Other (specify)

\section{MEDICAL DIRECTOR OR CHIEF}

a. Is there a medical director in addition to the administrator $\square$ or are they the same?

b. Work status: (1) Full-time, paid

(2) Part-time, paid

(3) Unpaid

c. Medical specialty: (check one)

$\square$ (1) Pediatrics

$\square$ (2) Other (specify)

$\square$ (3) None

d. Diplomate (certified by Am. Exam. Boards of Med. Spec.)

Yes $\square \quad$ No $\square$

14. NUMBER OF STAFF MEMBERS

a. General medical staff (incl. consultants)

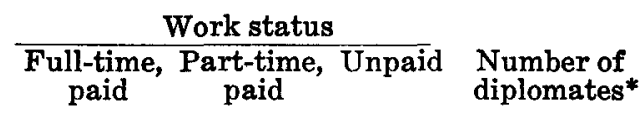

(1) Pediatricians

(2) General practitioners

(3) Internists

(4) Otolaryngologists

(5) Surgeons

(6) Neurological surgeons

(7) Orthopedic surgeons

(8) Other (specify)

*Diplomates are holders of certificates from Am. Exam. Boards of Med. Spec.

b. Medical house staff:

Total Number assigned number to children only

(1) Interns (1st year)

(2) Ass't residents (2nd year)

(3) Residents (3rd year or longer)

(4) Other (explain)

c. Nursing staff :

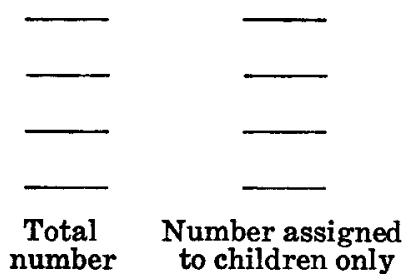

(1) Graduate nurses

(2) Student nurses

(3) Custodians*

*Include practical nurses, attendants, nurses' aides, maids and orderlies.

d. Auxiliary staff :

Work status of all $\underset{\text { paid }}{\text { Full-time, }} \underset{\text { paid }}{\text { Part-time, Unpaid }}$

(1) Occupational therapists

(2) Social workers

(3) Physiotherapists

(4) Other (explain)
Work status of number assigned to children only

Full-time, Part-time, Unpaid paid paid 


\section{INSTRUCTIONS TO THE FIELD AGENT FOR FILLING SCHEDULE I-H}

An entry must be made for each item. If there are items for which the hospital is not able to supply data, make a note to that effect through or by the item. All items requiring a "yes" - "no" answer are easily filled out. Where figures are asked for, the hospital staff may require a little time to get the data from their records and mail them to you; leave a stamped self-addressed envelope for this purpose.

Most of the items are self-explanatory. A few detailed instructions dealing with certain more complex items are given below for your assistance.

Data for one year.

Nonwhite.

2 Type of ownership.

5a Admission of children.

5e Length of residence......

$5 f$ Economic status.

6e Large wards.

7 Patient service data....

7c Cost per patient-day....

11. Laboratory facilities....

13. Medical director.

14. Staff members.

14a General medical staff..

Ask your informant to select the last year for which the hospital has complete data. Use this same year for answering all questions that deal with data on an annual basis (items $7 a-c$ and $9 a$ ).

Include Negro and other nonwhite races.

Check one item only. All church operated or affiliated institutions check (6). Include non-profit corporation under (7).

Fill one copy of this schedule items 1-4 for each contagious hospital or institution, but only for those admitting children should the schedule from item $5 \mathrm{~b}$ on be filled.

Explain if patient has to be resident of State or community for any period of time prior to admission.

Explain if patient has to declare income level or inability to pay for service.

If there are wards of 10 or more beds, state number of wards (not number of beds on these large wards).

The answers to all parts of this question should be entered carefully. These items are fundamental data used as the basis for other calculations.

Patient-day expense for in-patients is desired here. It is obtained by dividing total operating expenses for in-patients by total number of patient days reported in item $\mathrm{Tb}(1)$ (all patients).

If available in this hospital, make no entries for "readily available elsewhere".

Even if the administrator and the medical director are the same person, fill items 12 and 13.

In institutions employing the services of religious orders, entries should be made as though the positions indicated were filled by full-time or part-time paid workers, whether or not they received remuneration.

A full-time employee is one who works full time in the hospital even though he devotes part of his time to several jobs within the hospital during different periods of the day or night. A part-time employee is one who does not work full time for the hospital.

Be sure to include under the proper classification the medical director and the administrator if he is a physician.

After the schedule is complete, review it thoroughly for omissions and inconsistencies. Sign your name below in order that any questions that arise at a later date may be referred to you, and return the schedule promptly to the State office. Leave a duplicate completed copy at the hospital for their files, if they desire.

Name of field agent:

Return this schedule to: 


\section{ACADEMY OF PEDIATRICS STUT'0Y OF CHILD HEALTH SERVICES}

Schedule I-J. Orthopedic Hospitals

Date

Code No.

1. NAME AND LOCATION OF HOSPITAL

Name

Street and number

City or town County State

2. TYPE OF OWNERSHIP OR CONTROL (check one)

\section{Governmental}

(1) Federal

(2) State

(3) City, town, village

or other municipality

(4) County

(5) City-County
Non-Governmental

Non-profit

(6) Church

(7) Non-profit association

$\square$ (8) Individual

$\square$ (9) Partnership

$\square$ (10) Corporation

3. ACCREDITATIONS AND APPROVALS (check)

a. American Medical Association Registry: Yes $\square$ No

b. American College of Surgeons :

(1) Full approval $\square$

(2) Provisional approval

(3) Not approved

4. MEDICAL SCHOOL AFFILIATION

a. Is the hospital affiliated with a medical school?

If $Y E S$, check purpose:

(1) Instruction undergraduate students

(2) Postgraduate training

(3) Research and investigation

(11) Other (explain)

\section{DMISSION OF CHILDREN}

a. Are children admitted?

If $N O$, no further items on this schedule are to be filled.

Yes $\square \quad$ No $\square$

\section{If $Y E S$,}

b. Are children admitted from outside the State?

Yes $\square \quad$ No $\square$

c. Are children admitted from -
(1) Entire State
(2) Section of State only
(3) County or local community only

Yes No

Yes $\square \quad$ No $\square$

d. Check whether services for children are available to:
(1) White only
(2) White and nonwhite
or (3) Nonwhite only

e. Is service restricted by policy or regulation as to length of residence?

If $Y E S$, explain

f. Is service restricted by policy or regulation as to economic status?

If $Y E S$, explain

g. Earliest age for admission

Yes $\square \quad$ No $\square$

Yes $\square \quad$ No $\square$ years

6. ACCOMMODATIONS FOR CHILDREN

a. Give present number of beds actually set up for in-patient care, including children's cribs but excluding bassinets in newborn nursery -
(1) For all patients
(2) For children only

b. Are children entirely separated from adult patients (that is, are permanent $\quad$ Yes No facilities set aside for exclusive use of children?)

If $N O$, what provisions are there for children?

c. Are infants separated from older children?

d. Is there separation of nonwhite from white children?

e. Are there large wards ( 10 or more beds) for infants or children?

If $Y E S$, give number of wards (1) 10 to 20 beds

(2) 20 or more beds 
7. PATIENT SERVICE DATA FOR ONE YEAR

Data for year ended 194

(Same year to be used for all annual data requested.)

a. Summary of services rendered Number of patients :

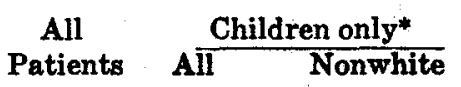

(1) Remaining in hospital beginning of year

(2) Admitted

(3) Total treated (1 and 2)

(4) Discharged (except deaths)

(5) Died

(6) Total discharged and died (4 and 5)

(7) Remaining end of year (3 minus 6)

*Specify upper age limit of patients classed as children:

b. Total patient days of service rendered:

(1) All patients

(2) All children

(3) Nonwhite children

c. Average cost per patient-day (i.e. per capita in-patient expense)

d. Number of children on waiting list for admission at present time

8. TYPES OF CHILDREN'S CASES ADMITTED

a. Check whether the following types of cases are admitted:

(1) Congenital defects of bones and joints

Yes No

(2) Cases requiring plastic surgery

(3) Poliomyelitis, chronic

(4) Osteomyelitis, chronic

(5) Tuberculosis of bone and joint

(6) Injuries (chronic)

(7) Other (specify)

9. FOSTER-HOME CARE

a. Does the hospital use foster homes for the care of children?

Yes $\square \quad$ No

b. Number of children so placed in year of report

10. CARE OF SICK CHILDREN

a. Are children with commuicable diseases:

(1) Kept on ward?

(2) Isolated in infirmary?

(3) Sent to communicable disease hospital?

b. Is there a routine isolation procedure for children on admission?

Yes $\square \quad$ No

If $Y E S$, check whether for: (1) 1 day only

(2) 2-7 days only

(3) 8 days or longer

11. OUT-PATIENT DEPARTMENT

a. Is there an out-patient department?

Yes $\square \quad$ No

If $Y E S$ :

b. Is there a children's clinic separate from clinics for adults?

c. Extent of service during one year:

Patients Visits

Yes $\square \quad$ No

(1) All types of service

(2) Services for children

12. LABORATORY FACILITIES

a. Check whether facilities for the following procedures are -

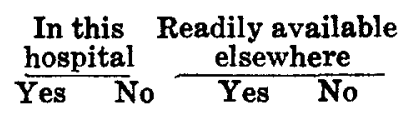

(1) Blood counts

(2) Sedimentation rates

(3) Urinalyses

(4) X-ray 
13. ADMINISTRATOR

a. Training (check one) :
(1) Medical doctor
(5) Reg. or grad. nurse: Nun, sister, mother
(2) Clergyman
(6) Reg. or grad. nurse: Not nun, sister, mother
(3) Osteopath
(7) Nun, sister, mother: Not reg. or grad. nurse
(4) Chiropractor
(8) Other (specify)

14. MEDICAL DIRECTOR OR CHIEF

a. Is there a medical director in addition to the administrator $\square$ or are they the same?

b. Work status: (1) Full-time, paid

(2) Part-time, paid

(3) Unpaid

c. Medical specialty: (check one)
$\square$ (1) Orthopedic surgery
(3) Other (specify)
(2) Pediatrics
(4) None

d. Diplomate (certified by Am. Exam. Boards of Med. Spec.)

Yes $\square \quad$ No $\square$

15. NUMBER OF STAFF MEMBERS

a. General medical staff (incl. consultants) :

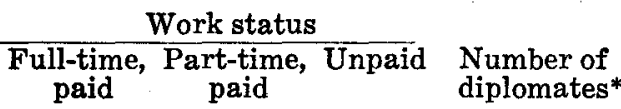

(1) Pediatricians

(2) General practitioners

(3) Orthopedic surgeons

(4) Internists

(5) Surgeons

(6) Psychiatrists

(7) Radiologists

(8) Neurologists

(9) Plastic surgeons

(10) Otolaryngologists

(11) Other (specify)

*Diplomates are holders of certificates from Am. Exam. Boards of Med. Spec.

b. Medical house staff :

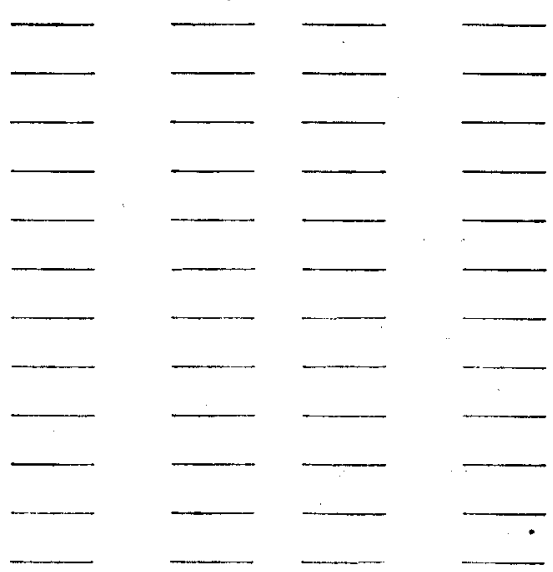

(1) Interns (1st year)

(2) Ass't residents (2nd year)

(3) Residents (3rd year or longer)

(4) Other (explain)

c. Nursing staff :

(1) Graduate nurses

(2) Student nurses

(3) Custodians*

*Include practical nurses, attendants, nurses' aides, maids and orderlies.

d. Auxiliary staff :

(1) School teachers

(2) Occupational therapists

(3) Social workers

(4) Physiotherapists

(5) Other (explain)
Total number

Number assigned to children only

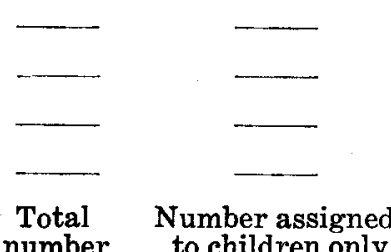

number to children only

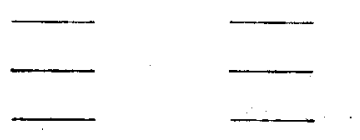

Work status of number asșigned to children only Full-time, Part-time, Unpaid paid paid 


\section{INSTRUCTIONS TO THE FIELD AGENT FOR FILLING SCHEDULE I-J}

An entry must be made for each item. If there are items for which the hospital is not able to supply data, make a note to that effect through or by the item. All items requiring a "yes" - "no" answer are easily filled out. Where figures are asked for, the hospital staff may require a little time to get the data from their records and mail them to you; leave a stamped self-addressed envelope for this purpose.

Most of the items are self-explanatory. A few detailed instructions dealing with certain more complex items are given below for your assistance.

Data for one year.

Nonwhite.

2 Type of ownership.

5a Admission of children...

5e Length of residence........

5f Economic status.

6e Large wards.

7 Patient service data....

7c Cost per patient-day.....

8 Type of cases

9 Foster-home care.

12 Laboratory facilities

14 Medical director.

15 Staff members

$+$

15a General medical staff..
Ask your informant to select the last year for which the hospital has complete data. Use this same year for answering all questions that deal with data on an annual basis (items $7 \mathrm{a}-\mathrm{c}, 9 \mathrm{~b}$, and $11 \mathrm{c}$ ).

Include Negro and other nonwhite races.

Check one item only. All church operated or affiliated institutions check (6). Include non-profit corporation under (7).

Fill one copy of this schedule item 1-4 for each orthopedic hospital, but only for those admitting children should the schedule from item $5 \mathrm{~b}$ on be filled.

Explain if patient has to be resident of State or community for any period of time prior to admission.

Explain if patient has to declare income level or inability to pay for service.

If there are wards of 10 or more beds, state number of wards (not number of beds on these large wards).

The answers to all parts of this question should be entered carefully. These items are fundamental data used as the basis for other calculations.

Patient-day expense for in-patients is desired here. It is obtained by dividing total operating expenses for in-patients by total number of patient days reported in item $7 \mathrm{~b}(1)$ (all patients).

Include hare-lip, cleft palate, and burn repairs among cases requiring plastic surgery.

This includes placing out in private homes of children who have been residents in the institution and/or those coming to the out-patient department.

If avalable in this hospital, make no entries for. "readily available elsewhere".

Even if the administrator and the medical director are the same person, fill items 13 and 14.

In institutions employing the services of religious orders, entries should be made as though the positions indicated were filled by full-time or part-time paid' workers, whether or not they received remuneration.

A full-time employee is one who works full time in the hospital even though he devotes part of his time to several jobs within the hospital during different periods of the day or night. A part-time employee is one who does not work full time for the hospital.

Be sure to include under the proper classification the medical director and the administrator if he is a physician.

After the schedule is complete, review it thoroughly for omissions and inconsistencies. Sign your name below in order that any questions that arise at a later date may be referred to you, and return the schedule prompt. ly to the State office. Leave a duplicate completed copy at the hospital for their files, if they desire.

Name of field agent:

Return this schedule to: 


\section{ACADEMY OF PEDIATRICS STUDY OF CHILD HEALTH SERVICES}

\section{Schedule II. Summary of Public Health Services Available for Children}

(Schedule II is to be filled for each health jurisdiction. For full-time health organizations the data for item 1 may be copied from form 9416, Report of Public Health Personnel, Facilities, and Services.)

1. DESCRIPTION OF HEALTH JURISDICTION
A. Name:
B. Type of Health Jurisdiction (check)
$\square$ (1) County
(2) City
$\square$ (3) Joint city-county
(4) State health district
$\square$ (5) Local health district

C. Within this health jurisdiction are there any communities not covered by this report?

If YES, list each community. (A separate report is to be filled for these.)

2. COUNTIES WITHIN THE HEALTH JURISDICTION ; also cities with $10,000+$ population with an independent health organization.

Component Counties* or Cities:

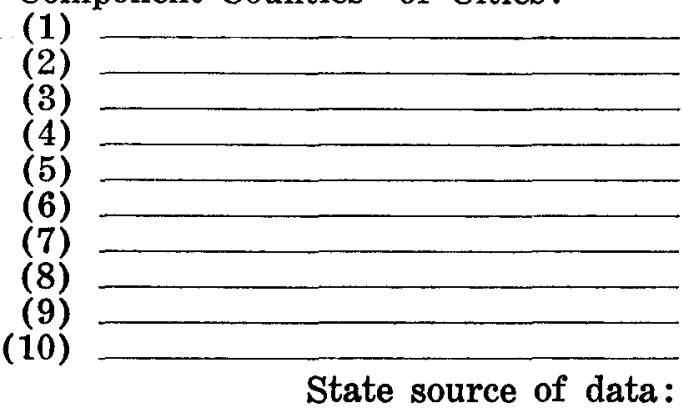

\section{Total population}$$
\text { White Nonwhite White Nonwhite }
$$
live births

No. of children attending school

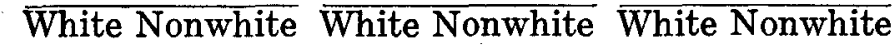

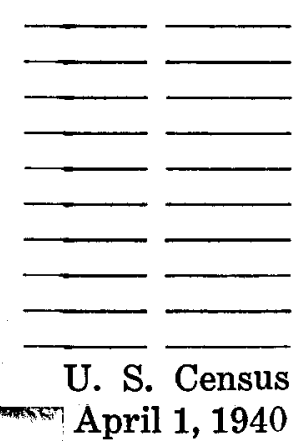

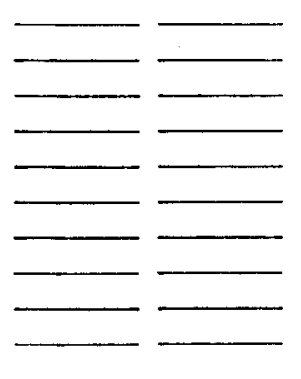

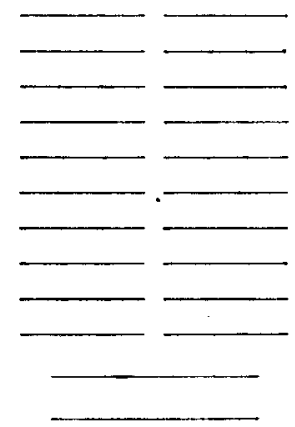

*The reporting county jurisdiction will include all cities of less than 10,000 , and the larger cities without independent health organizations.

\section{AGENCIES PROVIDING SPECIFIED TYPES OF CHILD HEALTH SERVICES}

On page 2 list separately each agency according to type of child health services; attach an extra sheet if more space is required. A detailed supplementary schedule is to be filled for each agency providing services as follows:

II-A Medical Well-Child Conferences

II-B Dental Services for Children

II-C Mental Hygiene Services for Children

II-D Health Services for Physically-Handicapped Children

II-E Communicable Disease Control

II-F School-Health Services

II-G Public Health Nursing Services

At least one copy of each of these schedules should be filled for each county or city within the health jurisdiction as listed in item 2. If the specified type of service is not available within the community, write " $O$ " at the top of the specific form. If services are provided outside the community, write at the top of the specific form the name of the agency providing such service to children of the community. 
3. AGENCIES PROVIDING SPECIFIED TYPES OF CHILD HEALTH SERVICES during report year -

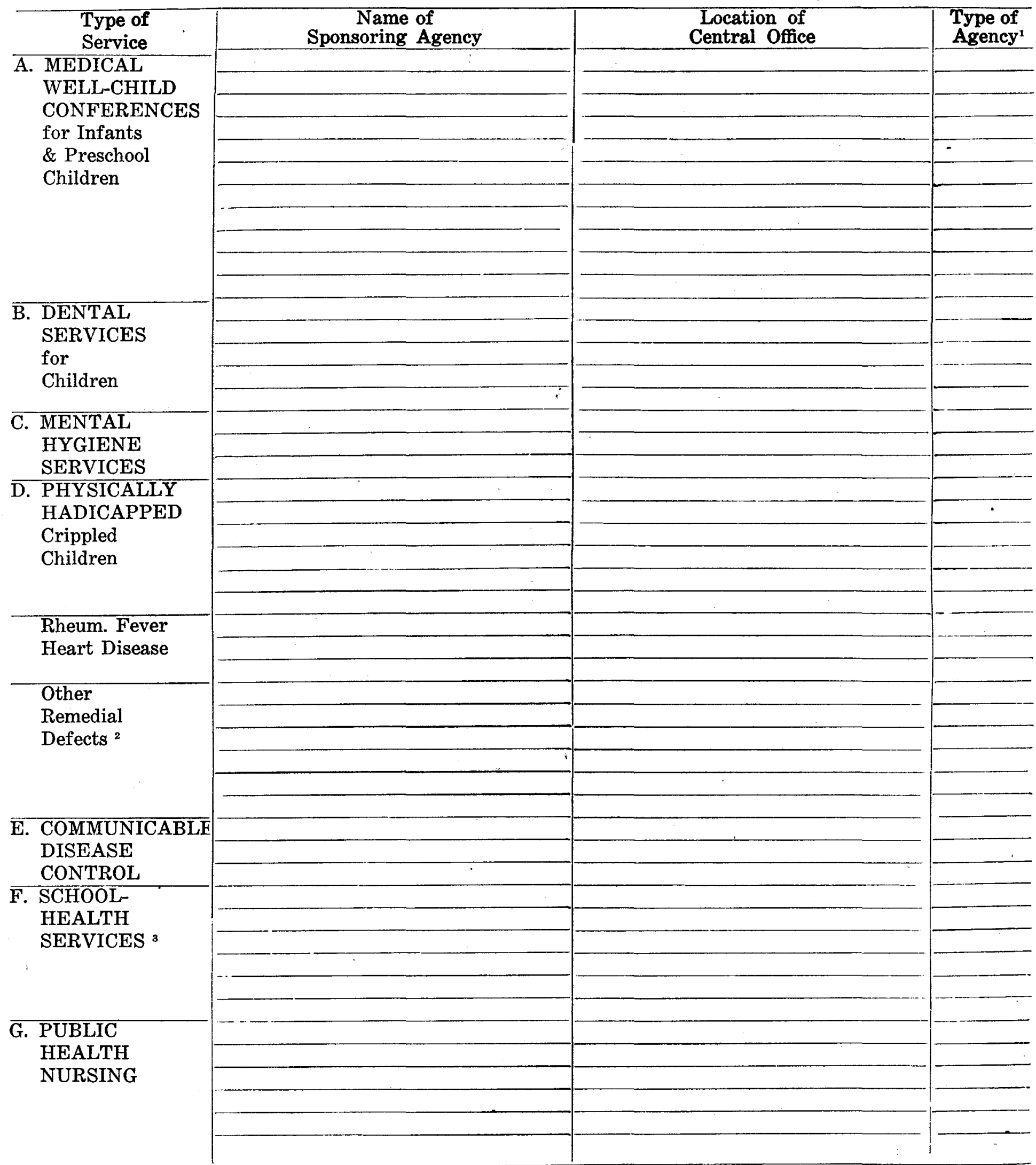

Code type of agency: X - Official health; Y - Other official; $\mathrm{Z}$ - Voluntary agency.

2 Medical corrective service for vision, hearing, speech, diabetes, allergy, etc.

3 Services other than those provided by agencies listed in the other items.

Return this sheet to: 


\section{ACADEMY OF PEDIATRICS STUDY OF CHILD HEALTH SERVICES}

\section{Schedule II-A. Medical Well-Child Conferences}

(Schedule II-A is to be filled for each agency listed in Schedule II, Item 3A.)

1. IDENTIFICATION OF AGENCY

A. Name of sponsoring agency

2. ADMISSION POLICY

A. Are children admitted from (1) Part of county (or city) only $\square$
(2) Entire county (or city) only
(3) Beyond county (or city)
B. Is service restricted by policy or regulation as to length of residence?

If YES, explain.

C. Is service restricted by policy or regulation as to economic status? If YES, explain

D. Is service provided without charge to -
(1) All children
(2) Some children $\square$

(3) No children $\square$

E. Must the patient be referred by a physician?

3. DATA ON MEDICAL WELL-CHILD CONFERENCES during year ended Yes $\square \quad$ No $\square$

(Include all services supplied or paid for by the sponsoring agency, whether in schools, or trailers.)

\section{Types of clinics}

A. Infants only

(1) White clinics

(2) Nonwhite clinics

(3) White \& nonwhite clinics

B. Preschools only

(1) White clinics

(2) Nonwhite clinics

(3) White \& nonwhite clinics

C. White infants \& pre-

schools combined

(1) Infants

(2) Preschools

D. Nonwhite infants \& preschools combined

(1) Infants

(2) Preschools

E. White \& nonwhite infants

\& preschools combined

(1) Infants

(2) Preschools

F. Total

\begin{tabular}{|c|c|c|}
\hline $\begin{array}{c}\text { No. of } \\
\text { sessions }\end{array}$ & $\begin{array}{l}\text { Usua } \\
\text { staff } \\
\text { Phys. }\end{array}$ & $\begin{array}{l}\text { al numbe } \\
\text { per sess } \\
\text { Nurses }\end{array}$ \\
\hline & & \\
\hline & & \\
\hline & & \\
\hline & & \\
\hline $\bar{x}$ & $\bar{x}$ & $x$ \\
\hline$x$ & $\bar{x}$ & $x$ \\
\hline $\bar{x}$ & $\times$ & $x$ \\
\hline$x$ & $x$ & $x$ \\
\hline $\bar{X}$ & $\bar{x}$ & $x$ \\
\hline$x$ & $x$ & $x$ \\
\hline & $x$ & $x$ \\
\hline
\end{tabular}

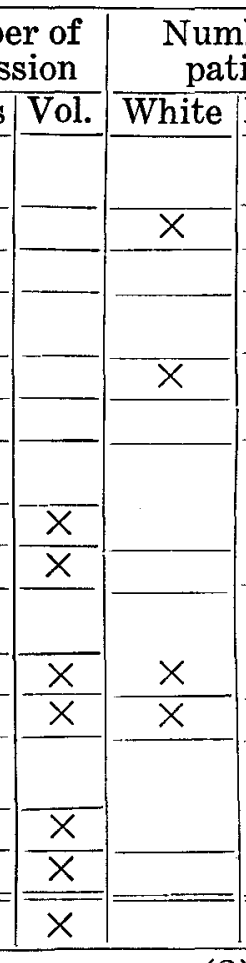

G. Upper age limits of persons classed as (1) Infants:

years

(2) Preschools: Number of
visits tients \begin{tabular}{|l|l}
\hline White & Nonwhite
\end{tabular}

4. LOCATION OF CENTERS OPERATED BY AGENCY

A. Name of town, or if large city give street address also.

(1)

(2)

(3)

(4)

(5)
Check whether services are provided to:

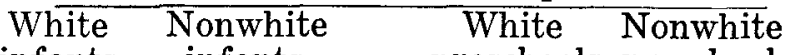

infants infants preschools preschools

Instructions: Fill at least one sheet of Schedule II-A for each county within the health jurisdiction; also for each city of 10,000 + population with an independent health organization. If medical well-child conferences are not available within the community, write " $O$ " at the top of the form. If services are provided outside the community, write at the top of the form the name of the agency outside the community providing such services to children of the community. Nonwhite includes Negro and other nonwhite races.

Item 2B. Explain if child has to be resident of State or community for any period of time prior to admission.

Item 2C. Explain if family has to declare income level or inability to pay for service.

Items 5-7 If policy and service are not uniform for all centers, fill out a separate sheet for items 5, 6 and 7 for each center. If practice is uniform, one sheet will suffice for all centers. 
5. TYPE OF SERVICE (other than public health nursing)

A. Are infants weighed on each visit? Yes $\square \quad$ No $\square$

B. How many examinations (without clothes) are usually given per child per year?

(1) Infant under 1 year

(2) Child 1-5 years

C. Are immunizations performed routinely for:

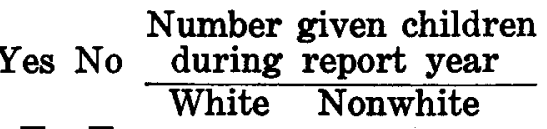

(1) Smallpox

(2) Diptheria - (1) infants under 1 year only

(2) Preschool children

(3) Whooping cough - (1) Infants under 1 year only

(4) Typhoid

(2) Preschool children

(5) Tetanus

D. Are the following tests:

Check one

\begin{tabular}{ccc}
\hline $\begin{array}{c}\text { Given } \\
\text { routinely }\end{array}$ & $\begin{array}{c}\text { As indicated Not } \\
\text { only }\end{array}$ & $\begin{array}{c}\text { given } \\
\square\end{array}$ \\
$\square$ & $\square$ & $\square$ \\
$\square$ & $\square$ & $\square$ \\
$\square$ & $\square$ & $\square$
\end{tabular}

(1) Schick

(2) Tuberculin

(3) Serologic test for syphilis -..

E. Is advice to mother given by physicians:

Yes No

(1) On formulae and feeding

(2) On care and training

F. Are the following special services available:

(1) Consultant service by nutritionists: to staff

to parents

(2) Consultant service by psychologist or psychiatrist: to staff

to parents

G. If sick children come to clinic, check procedure:
$\square$ (1) Sent home
(3) Treated
(2) Isolated until seen by doctor
(4) Referred elsewhere

H. Are clinic sessions (1) Held for well-child supervision only $\square$

(2) Combined with maternity clinics $\square$ (3) Combined with tbc., VD., or other clinics

6. PUBLIC HEALTH NURSING SERVICE

A. Is the public health nursing service for the conferences provided by -

Yes No

(1) Agency shown in item 1A

(2) Another agency (specify)

B. Is public health nursing follow-up service in the home available to the following children attending well-child conferences?
Infants
Yes No
Preschool children
Yes No
(1) White
$\square \quad \square$
(3) White
(4) Nonwhite

C. Are nursing conferences for well-child supervision held as well as medical conferences?

Yes $\square \quad$ No

7. PHYSICIANS SERVING IN CENTER during report year

Data for each physician to be entered on a separate line.

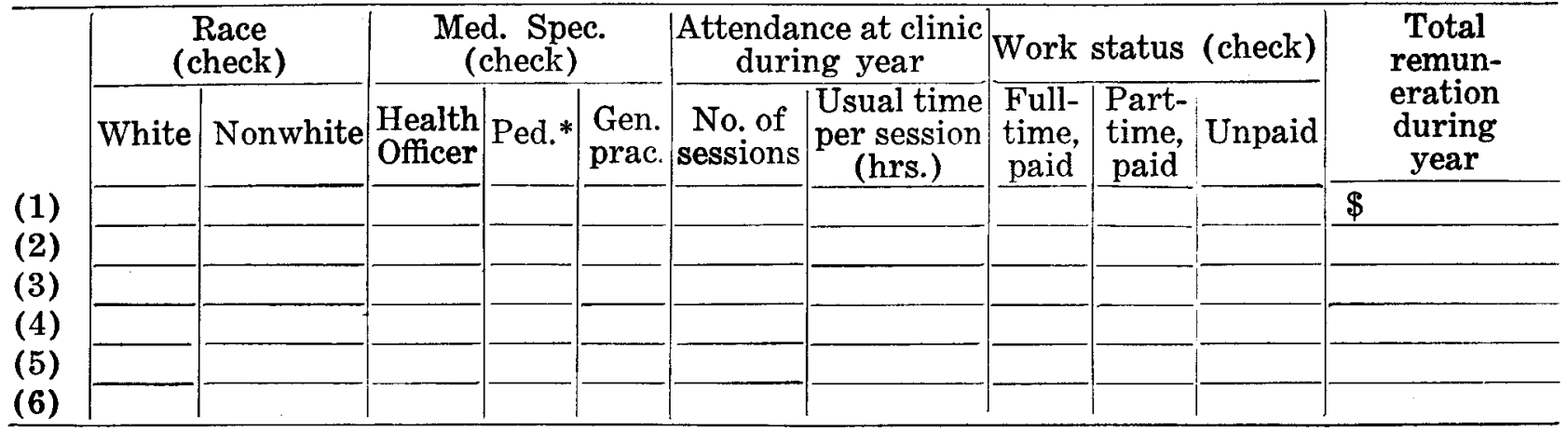

*A physician who limits his practice to pediatrics only. 


\section{ACADEMY OF PEDIATRICS STUDY OF CHILD HEALTH SERVICES Schedule II-B. Dental Services for Children}

(Schedule II-B is to be filled for each agency listed in Schedule II, Item 3 B.)

\section{IDENTIFICATION OF AGENCY}

A. Name of sponsoring agency

\section{ADMISSION POLICY}

A. Are children admitted from (1) Part of county (or city) only $\square$
(2) Entire county (or city) only
(3) Beyond county (or city)

B. Is service restricted by policy or regulation as to length of residence? _.. Yes $\square$ No If YES, explain

C. Is service restricted by policy or regulation as to economic status? If YES, explain

D. Is service provided without charge to -
(1) All children
(2) Some children
(3) No children

E. Must the patient be referred by a dentist? Yes No

F. What are the age limits for admission? From thru years

3. DATA ON DENTAL SERVICES during one year (year ended 194__) (Include all services supplied or paid for by the sponsoring agency, whether in clinics, schools, trailers or private offices.)

A. Number of dentist-hours, total

(1) For white children

(2) For nonwhite children

B. Dental services (other than examinations)

(1) Number of different patients given service

Preschool children White Nonwhite

School children

(2) Total patient-visits

(3) Number of services of each type, total

a. Extractions, permanent teeth

b. Extractions, deciduous teeth

c. Fillings, permanent teeth

d. Fillings, deciduous teeth

e. Prophylaxes

f. Orthodontic visits

g. Other (explain)

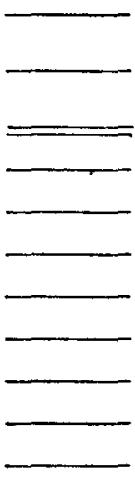

C. Dental examinations

(1) Total no. oral examinations or inspections

a. By dentists

b. By others
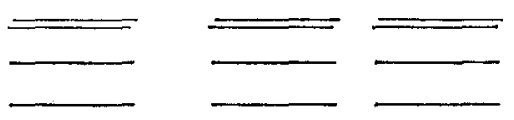

Instructions: Fill at least one sheet of Schedule II-B for each county within the health jurisdiction; also for each city of $10,000+$ population with an independent health organization. If dental services are not available within the community, write " $O$ " at the top of the form. If services are provided outside the community, write at the top of the form the name of the agency providing such services to children of the community.

Nonwhite includes Negro and other nonwhite races.

Item 2B. Explain if child has to be resident of State or community for any period of time prior to admission.

Item 2C. Explain if family has to declare income level or inability to pay for services.

Item 3A. Total time for all services by dentists, including examinations.

Item 3B (1) Include patients given dental service, not those receiving only examinations or inspections. 
Heaith juris. Code No.

\section{ACADEMY OF PEDIATRICS STUDY OF CHILD HEALTH SERVICES}

\section{Schedule II-C. Mental Hygiene Services for Children}

(Schedule II-C is to be filled for each agency listed in Schedule II, item 3C.)

\section{IDENTIFICATION OF AGENCY}
A. Name of sponsoring agency

2. ADMISSION POLICY

A. Are children admitted from (1) Part of county (or city) only
(2) Entire county (or city) only
(3) Beyond county (or city)

B. Is service restricted by policy or regulation as to length of residence? _... Yes $\square$ No $\square$ If YES, explain

C. Is service restricted by policy or regulation as to economic status? Yes $\square \quad$ No If YES, explain

D. Is service provided without charge to -
(1) All children
(2) Some children
(3) No children $\square$

E. Must the patient be referred by a physic an?

F. What are the age limits for admission? From Yes No $\square$

G. Is service provided to - (1) White children only $\square$ thru years

(2) White and nonwhite children

(3) Nonwhite children only

3. DATA ON MENTAL HYGIENE SERVICES during one year (year ended 194 (Include all services supplied or paid for by the sponsoring agency, whether in clinics, schools, trailers or private offices.)

Children

A. Number of different patients seen White Nonwhite

B. Total patient-visits

C. Number of days of clinic service (If $1 / 2$ days of service are given, report as equivalent full days.)

D. Types of children's cases admitted and for what purpose
Ydmitted
(1) Mental deficiencies
(2) Psychoses
(3) Psychoneuroses and neurotic traits
(4) Convulsive disorders
(5) Delinquents
(6) Behavior problems
If admitted, check whether for - Diagnosis and/or treatment

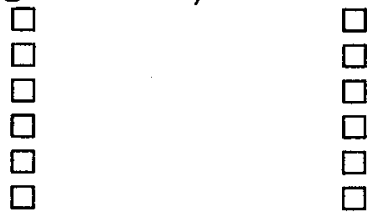
$\square$
$\square$
$\square$
$\square$
$\square$
$\square$

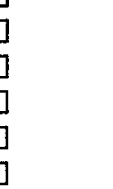

4. PROFESSIONAL STAFF SERVING AT CLINICS during report yedx

A. Number of -

(1) Psychiatrists

(2) Psychologists

(3) Pediatricians

(4) Social workers

(5) Others (specify)

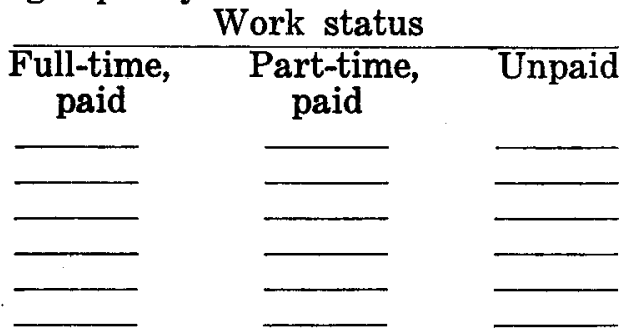

5. SERVICE TO OTHER AGENCIES

A. Does the agency provide service to the following:

(1) Other public health clinics

(2) Schools

(3) Hospitals and institutions

(4) Welfare organizations (adoption, placement, etc.)

(5) Juvenile courts Instructions: Fill at least one sheet of Schedule II-C for each county within the health jurisdiction; also for each
city of $10,000+$ population with an independent health organization. If mental hygiene services are not available within the community, write " $O$ " at the top of the form. If services are provided outside the community write at the top of the form the name of the agency providing such services to children of the community.

Nonwhite includes Negro and other nonwhite races.

Item 2B. Explain if child has to be resident of State or community for any period of time prior to admission.

Item 2C. Explain if family has to declare income level or inability to pay for service. 


\section{4 \\ ACADEMY OF PEDIATRICS STUDY OF CHILD HEALTH SERVICES \\ Schedule II-E. Communicable Disease Control}

(Schedule II-E is to be filled for each agency listed in Schedule II, item 3E.)

\section{IDENTIFICATION OF AGENCY}

A. Name of sponsoring agency:

2. DATA ON IMMUNIZATIONS during one year (year ended 194_)

A. Type of immunization:

(1) Smallpox

(2) Diptheria: Infants under 1 year only.

Children 1 thru 14 years.

(3) Whooping cough: Infants under 1 year only

(4) Typhoid

Children 1 thru 14 years

*Are any of these immunizations reported on Schedule II-A, Medical wellchild conferences?

B. Check whether immunizations are given by -
(1) Physician
(2) Registered nurse
(3) Medical student
(4) Student nurse
(5) Volunteers, aides
(6) Other (specify)

3. SPECIAL SERVICES BY OFFICIAL HEALTH AGENCY (to be filled only by official agency)

A. Is there on the staff, either full-time or part-time, of the official health agency a physician who has specialized training or experience in communicable disease diagnoses and treatment? Yes No $\square$

\section{If YES,}

(1) Are the services of this physician available without cost on a consultant basis to any physician in the health jurisdiction? Yes $\square \quad$ No

B. Number of times during report year the official health agency has been called in consultation by a private physician to assist in diagnosis of a case of communicable disease

C. Number of cases of communicable disease reported during year -

White Nonwhite

White Nonwhite

(1) Smallpox

(2) Diptheria
(3) Whooping cough

(4) Typhoid, paratyphoid

Instructions: Fill at least one sheet of Schedule II-E for each county within the health jurisdiction; also for each city of $10,000+$ population with an independent health organization. If communicable disease control services are not available within the community, write " $O$ " at the top of the form. If services are provided outside the community, write at the top of the form the name of the agency providing such services to children of the community.

Nonwhite includes Negro and other nonwhite races.

Item 2A. If data for children are not separated from those for adults, enter the total figures but change the column headings to read " adults and children." 


\section{ACADEMY OF PEDIATRICS STUDY OF CHILD HEALTH SERVICES \\ Schedule II-F. School-health Services}

(Schedule II-F is to be filled for each agency listed in Schedule II, item $3 \mathrm{~F}$. Certain health services for school children are also included on the schedules for dental, mental hygiene, physically-handicapped, communicable disease, and nursing services.)

\section{IDENTIFICATION OF AGENCY}
A. Name of sponsoring agency

2. MEDICAL EXAMINATIONS during one year (year ended

A. Are medical examinations by a physician done on -

(1) All pupils once a year

(2) Certain grades once a year If YES, specify grades examined

(3) Referrals by teachers or nurses

(4) Athletic teams

\begin{tabular}{|c|c|c|c|}
\hline \multicolumn{2}{|c|}{ White children } & \multicolumn{2}{|c|}{ Nonwhite children } \\
\hline Yes & No & Yes & No \\
\hline$\square$ & $\square$ & $\square$ & $\begin{array}{l}\square \\
\square\end{array}$ \\
\hline $\begin{array}{l}\square \\
\square\end{array}$ & $\begin{array}{l}\square \\
\square\end{array}$ & $\begin{array}{l}\square \\
\square\end{array}$ & $\begin{array}{l}\square \\
\square\end{array}$ \\
\hline
\end{tabular}

3. SPECIAL EXAMINATIONS during report year

A. Vision tests:

(1) By teacher

(2) By nurse or technician

(3) By doctor

If YES, a. Type of test: Snellen

b. Frequency

Other

c. Grades tested

B. Hearing tests :

(1) By teacher

(2) By nurse or technician

(3) By doctor

If YES, a. Type of test: Audiometer $\square$

b. Frequency

c. Grades tested

C. Test for tuberculosis

Other

(1) X-ray

(2) Tuberculin test

If YES, grades tested

4. SPECIAL SERVICES during report year

A. Are the services of the following available:

(1) Nutritionist

(2) Psychologist or psychiatrist

(3) Health educator

(4) Sanitarian

(5) Speech teacher

5. MEDICAL STAFF SERVING during report year

A. Number of staff members, total

(1) General practitioners

(2) Pediatricians

(3) Other (Specify)

B. Number of staff members by work status:

(1) Full-time, paid

(2) Part-time, paid

(3) Unpaid

(over)

Instructions: Fill at least one sheet of Schedule II-F for each county within the health jurisdiction; also for each city of $10,000+$ population with an independent health organization. If school health services are not available within the community, write " $O$ " at the top of the form. If services are provided outside the community, write at the top of the form the name of the agency outside the community providing such services to children of the community.

Nonwhite includes Negro and other nonwhite races. 
6. NURSING SERVICE during report year

A. Number of nurses employed by this agency giving any service to schools

B. If nursing service is supplied by another agency, identify agency

C. Check whether the following types of services by nurses are available:

White children Nonwhite children

(1) Inspection of pupils

(2) Special tests, as vision or hearing

(3) Assistance with medical examinations

(4) Conference with teachers and parents

(5) Health counseling to pupils

(6) Home visits: Attendance

(7) Home visits: Other than attendance

(8) Participation in health education program: classroom teaching

(9) Participation in health education program: curriculum construction and advisory service to teachers

7. SCHOOLS IN PROGRAM during report year

Yes No Yes No

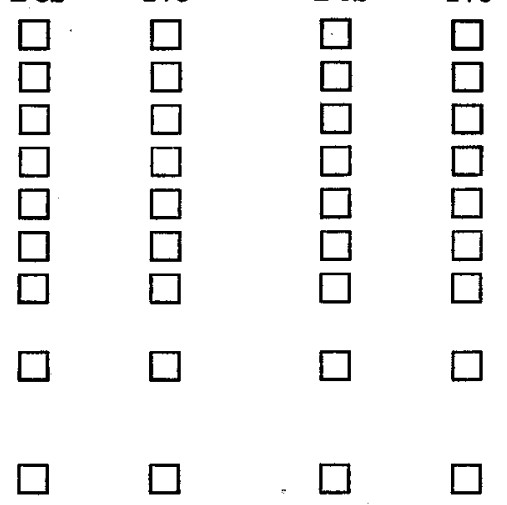

White Nonwhite children children

A. Number of children enrolled in schools in program, total

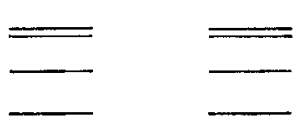

(2) Private and parochial schools

B. In any of these same schools, is any other agency responsible for school health services (as listed in items 2, 3, and 4 of this schedule) Yes $\square \quad$ No If $Y E S$, see that a separate copy of Schedule II-F is filled for that agency. 


\section{ACADEMY OF PEDIATRICS STUDY OF CHILD HEALTH SERVICES}

\section{Schedule II-G. Public health Nursing Services}

(Schedule II-G is to be filled for each agency listed in Schedule II, Item 3G.)

1. IDENTIFICATION OF AGENCY

A. Name of sponsoring agency:

B. Area served: (1) Part of county (or city) only $\square$ (2) Entire county (or city) only

(3) Beyond county (or city)

2. NURSES ENGAGED IN PUBLIC HEALTH NURSING ACTIVITIES during one year (year ended $194 \_$)

Number of nurses

A. Total number

B. Work status :

(1) Full-time, paid

(2) Part-time, paid

(3) Unpaid

C. Preparation in an approved program of Public Health Nursing:

(1) Number who completed one academic year

(2) Number who completed less than one year

(3) Number with no special training

D. Activities of nurses:

(1) Number engaged only in generalized programs

(2) Number engaged in special programs in which adults and children are cared for

(3) Number engaged in children's programs only

(4) Number engaged in adult's programs only

3. EXTENT OF SERVICE during report year

A. Home visits by nurses for supervision, morbidity and crippled children:

(1) Total number of visits to children and adults

(2) Total number of visits to children

(3) To infants

(4) To preschool children

(5) To school children

White Nonwhite

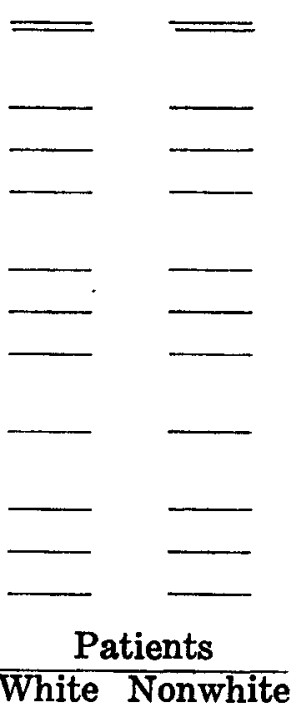

4. TYPES OF SERVICE

A. Check whether the following types of services are rendered by nurses.

(1) Assistance in clinics: Well-child conferences

(2) Assistance in clinics: Physically-handicapped

(3) Assistance in clinics: Other clinics

(4) Home visits: Health supervision

(5) Home visits: Bedside nursing

(6) Home visits: Home delivery service

(7) School service

(8) Children in group care, as day nurseries

(9) Classes for mothers

Instructions: Fill at least one sheet of Schedule II-G for each county within the health jurisdiction; also for each city of $10,000+$ population with an independent health organization. If public health nursing services are not available within the community, write " $O$ " at the top of the form. If services are provided outside the community, write at the top of the form the name of the agency providing such services to children of the community.

Nonwhite includes Negro and other nonwhite races. 


\section{ACADEMY OF PEDIATRICS STUDY OF CHILD HEALTH SERVICES}

\section{Schedule III-A. Physicians in Private Practice}

Date

Code No.

1. Are you engaged in private practice? Yes $\square \quad$ No $\square$ If NO, state reason turn the schedule.

2. Do you see newborns, infants or children under 15 years of age:

a. In your general practice? Yes $\square \quad$ No $\square$

b. As a consultant in your specialty? Yes $\square \quad$ No $\square$

3. Hospital training after graduation from medical school Yes $\square$ No $\square$ If $Y E S$ : a. No. of years

b. Length of pediatric service yrs. mos.

4. Postgraduate training in pediatrics, including refresher courses (exclude internship and residency) If YES, a. Length of time weeks months. b. School Yes $\square \quad$ No $\square$

5. Type of practice (check one)

$\square$ a. Limited to one specialty; state specialty

$\square$ b. Not limited but special interest; state branch

$\square$ c. No specialty.

6. Do you care for child patients for-

Yes No

a. Major surgery?

b. Tonsillectomies?

c. Feeding problems?

7. How many times in the past year have you called in a pediatrician to see a sick child with you?

8. How far from your office is the nearest hospital that admits child patients? (check only one.)

$\square$ a. Same city or town

b. Not same city or town, but less than 25 miles

$\sqsupset$ c. 25 to 50 miles

$\square$ d. 50 to 100 miles

$\sqsupset$ e. 100 or more miles

9. Are you permitted to take care of your own child patients in this hospital? .... Yes $\square$ No $\square$

10. During the past month how many hours did you spend:

a. In child-health conferences or clinics?

b. In school-health services?

c. In other medical activities besides private practice, as teaching, out-patient clinics, ward rounds (exclude medical meetings)?

11. One day record only. Enter the number of visits made by you on

Please report for this specific day, regardless of whether you consider it typical of your practice. You may wish to make remarks on the reverse of this sheet. Include only your private practice, not patients seen on hospital ward rounds. Consider delivery as newborm and adult, unless the care of the baby is turned over to another physician.

Number of visits to:

Location of Visit Office Home Hospital Visits

(1) Newborns, infants under 1 mo.

(2) Children 1 mo. to $1 \mathrm{yr}$.

(3) Children 1 - 5 yrs.

(4) Children 6 - 14 yrs.

(5) Persons 15 or more years *Children seen for health examination, immunization, advice on feeding, etc.
Of the total visits number of well* children seen

Please return this sheet to: 


\section{ACADEMY OF PEDIATRICS STUDY OF CHILD HEALTH SERVICES}

\section{Schedule III-B. Pediatricians in Private Practice}

Date.

Code No.

1. Hospital training after graduation from medical schoolLength of service

a. Intern yrs. mos. Length of pediatric service

b. Assistant resident yrs mos.

c. Resident

2. Postgraduate training in pediatrics, including refresher courses (exclude internship and residency)

If YES, a. Length of time weeks months.

b. School

3. Do you specialize in one of these pediatric branches?

Yes No
a. Pediatric - allergy
b. Pediatric - psychiatry and/or behavior problems
c. Pediatric - cardiology
d. Other (specify)

4. Do you care for your patients for-

a. Fractures?

b. Tonsillectomies?

c. Minor surgery?

5. How many times in the past year have you been called to see a sick child in consultation with a general practitioner?

6. How many times do you customarily see an infant during first year of life for feeding and health supervision?

7. How far from your office is the nearest hospital that admits child patients? (check only one)
a. Same city or town
c. 25 to 50 miles
b. Not same city or town, but
d. 50 to 100 miles
less than 25 miles
e. 100 or more miles

8. Are you permitted to take care of your own patients in this hospital -

a. As private patients?

Yes No

b. As semi-private patients?

c. As ward patients?

9. How far is the nearest hospital to which you can send children with communicable disease? (check only one)
a. Same city or town
c. 25 to 50 miles
b. Not same city or town, but
less than 25 miles
d. 50 to 100 miles
e. 100 or more miles

10. Check whether facilities for the following procedures are- In your
office
Yes No
a. Blood counts
b. Urinalyses
c. Blood chemistry
d. Culture for hemolytic streptococcus
e. X-ray and/or fluoroscopy

f. Electrocardiography

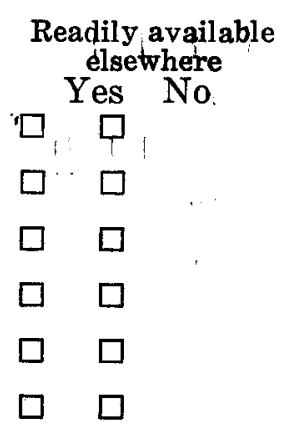


11. Do you care for any children as of this date on a prepaid basis?

If YES -

a. Number of children cared for on prepaid basis as of this date

b. Payments for such service made (check) :

(1) yearly

(2) quarterly

(3) monthly $\square$

c. Service includes :

(1) All office care

Yes No

(2) Office care for well-child supervision \& immunization only

(3) Telephone consultations

(4) Home visits

(5) Hospital visits

(6) Other (specify)

d. Is this service provided to children (check) :
(1) Under 1 year only?
(2) Under 2 years only?
(3) Of all ages?

If $\mathrm{NO}$ -

e. Are you opposed to the principle of prepayment?

Yes $\square \quad$ No $\square$

Remarks

12. Do you practice as a member of a group? Yes $\square$ No $\square$ If YES -

a. Number of physicians in group, total

(1) Number of pediatricians

Please state below names of other pediatricians associated with you.

If NO -

b. Are you opposed to the principle of group practice? Yes $\square \quad$ No $\square$

Remarks

13. What is the upper age limit of your patients? thru years

14. How many children have you seen in your private practice during the past year? If data are not available from your records, please estimate and so mark.

15. Please keep a record of the number of different patients seen during four consecutive weeks. Count each person only once. Be sure to enter number of patients here; number of visits during the same period is requested in item 16.

4-week period from thru 194

Number of patients, total

(1) Infants under $1 \mathrm{mo}$.

(2) Infants $1 \mathrm{mo}$. to $1 \mathrm{yr}$.

(3) Children 1-5 yrs.

(4) Children 6-14 yrs.

(5) Persons $15+$ yrs.

16-17. For the same 4-week period enter in item 16 the number of visits made and in item 17 the number of telephone consultations and hours spent in health services. Page 3 provides space for a 1-week record for each item; four sheets are attached.

Please return all sheets to: 
16. Daily record of number of visits made during one week.

Week ended

Location of visit and age of patient (Private practice only) a. OFFICE

1. Infants under 1 mo. ........

2. Infants $1 \mathrm{mo}$. to $1 \mathrm{yr}$.

3. Children 1-5 yrs.

4. Children 6-14 yrs.

5. Persons $15+$ yrs. ..........

b. HOME, Patient's:

1. Infants under $1 \mathrm{mo}$.

2. Infants 1 mo. to $1 \mathrm{yr}$. ...

3. Children 1-5 yrs.

4. Children 6-14 yrs.

5. Persons $\mathbf{1 5}+$ yrs.

c. HOSPITAL (priv, practice)

1. Infants under $1 \mathrm{mo}$.....

2. Infants $1 \mathrm{mo}$. to $1 \mathrm{yr}$.

3. Children 1-5 yrs.

4. Children 6-14 yrs.

5. Persons $15+$ yrs.

\begin{tabular}{|c|c|c|c|c|c|c|c|c|c|c|c|c|c|}
\hline \multicolumn{2}{|c|}{ SUNDAY } & \multicolumn{2}{|c|}{ MONDAY } & \multicolumn{2}{|c|}{ TUESDAY } & \multicolumn{2}{|c|}{ WEDNESDAY } & \multicolumn{2}{|c|}{ THURSDAY } & \multicolumn{2}{|c|}{ FRIDAY } & \multicolumn{2}{|c|}{ SATURDAY } \\
\hline $\begin{array}{l}\text { SICK } \\
\text { PTS. }\end{array}$ & $\begin{array}{c}\text { WELL* } \\
\text { PTS. }\end{array}$ & $\begin{array}{l}\text { SICK } \\
\text { PTS. }\end{array}$ & $\begin{array}{c}\text { WELL* } \\
\text { PTS. }\end{array}$ & $\begin{array}{l}\text { SICK } \\
\text { PTS. }\end{array}$ & $\begin{array}{c}\text { WELL } \\
\text { PTS. }\end{array}$ & $\begin{array}{l}\text { SICK } \\
\text { PTS. } \\
\end{array}$ & $\begin{array}{l}\text { WELL* } \\
\text { PTS. }\end{array}$ & $\begin{array}{l}\text { SICK } \\
\text { PTS. }\end{array}$ & $\begin{array}{l}\text { Weul } \\
\text { PTs. }\end{array}$ & $\begin{array}{l}\text { SICK } \\
\text { PTS. }\end{array}$ & $\begin{array}{l}\text { WEIx * } \\
\text { Prs. }\end{array}$ & $\begin{array}{l}\text { SICK } \\
\text { PTS. }\end{array}$ & $\begin{array}{l}\text { WBLL" } \\
\text { Prs. }\end{array}$ \\
\hline
\end{tabular}
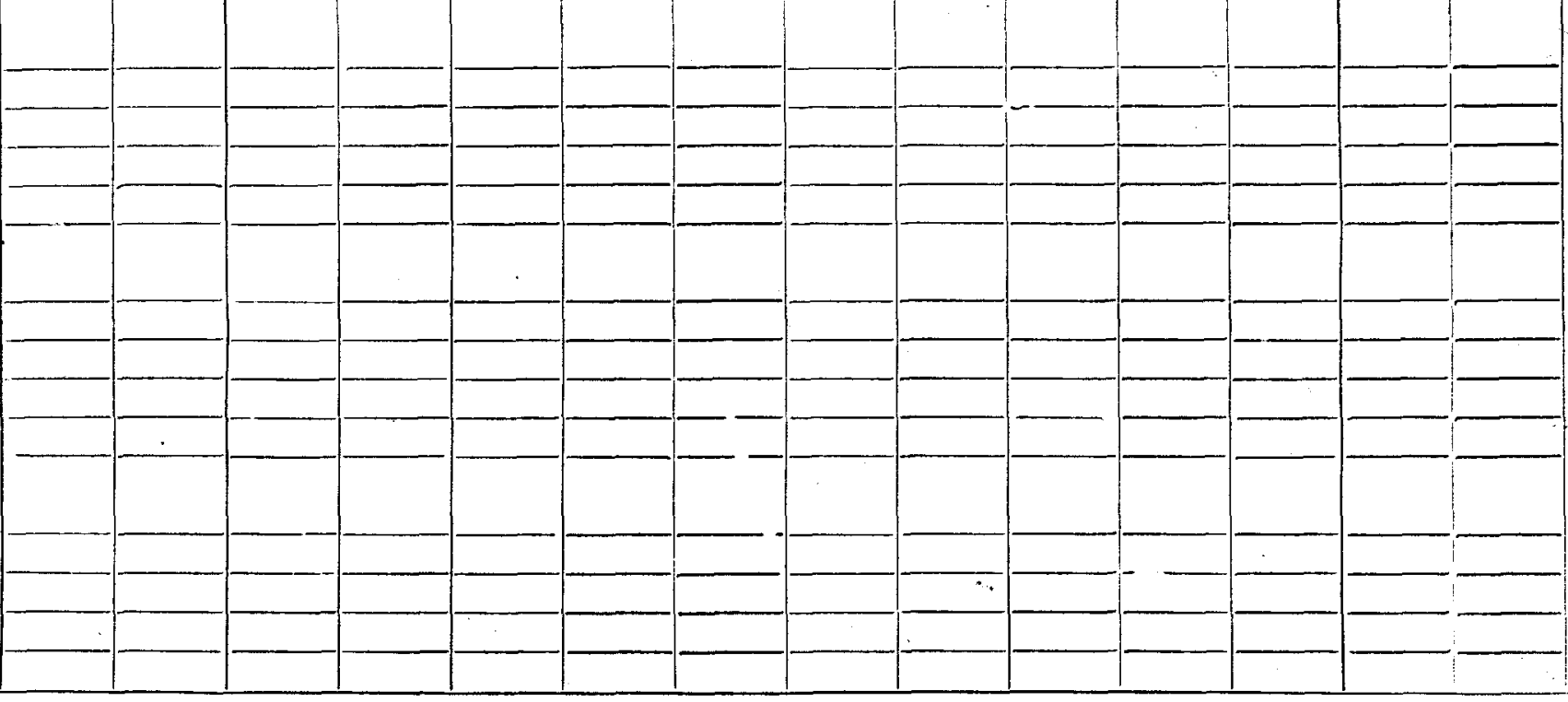

17. Daily record of number of telephone consultations and number of hours spent in health services during one week.

a. No. of telephone consulta-
tions with patients .........




\section{ACADEMY OF PEDIATRICS STUDY OF CHILD HEALTH SERVICES}

\section{Schedule III-C. Dentists in Private Practice}

Date

Code No.

1. Name and address of dentist:

Name

Street and number

City or town County State

2. Sex: Male $\square$ Female

3. Race: White $\square$ Other $\square$

4. Year of birth:

5. Graduation from dental school: a. School

b. Year

6. Are you engaged in private practice? Yes $\square \quad$ No $\square$ If $N O$, state reason If $N O$, you may stop here, but please return the schedule.

7. In your private practice do you see children under 15 years of age for service other than extractions or emergency?

8. Type of practice (check one)

$\square$ a. Limited to one specialty

$\square$ b. Not limited but special interest in one branch

口 c. No specialty

If " $a$ " or " $b$ ", check specialty:

$\square$ (1) Oral surgery

$\square$ (2) Orthodontics: Certified by Am. Board of Orthodontics : Yes $\square \quad$ No $\square$

$\square$ (3) Pedodontics

$\square$ (4) Peridontics: Certified by Am. Board of Peridontology : Yes $\square \quad$ No $\square$

口 (5) Prosthetics

9. Postgraduate training in pedodontics, including refresher courses If $Y E S$ : a. Length of time weeks. months b. School or agency

10. Number of office assistants, Total*

a. Secretary and office girls *If one person is used for $a$ and $b, b$ and

b. Chair assistants $c$, or a and c, please use brackets to so indicate.

c. Dental hygientists

11. Number of hours during past four weeks spent in dentistry, Tota

a. Private practice

b. Preschool or school dental services

c. Other dental activities as teaching, out-patient clinics, institutional work (exclude dental meetings)

12. One day record only. Enter the number of patients seen by you in private practice on

Please report for this specific day, regardless of whether you consider it typical of your practice. You may wish to make remarks on the reverse of this sheet.

\begin{tabular}{l|l|l|l} 
& $\begin{array}{c}\text { Children } \\
\text { under } 6 \text { years }\end{array}$ & $\begin{array}{c}\text { Children } \\
6-14 \text { years }\end{array}$ & $\begin{array}{c}\text { Persons } \\
\text { a. Number of patients seen }\end{array}$ \\
b. Number of dentist-hours & & \\
c. Number of services, total .. & & \\
(1) Extractions & & \\
(2) Fillings & & \\
(3) Other & & \\
\hline
\end{tabular}

Please return this sheet to: 


\section{BIBLIOGRAPHY}

Crittenden, C. B: "The Problem of Preventable Waternal Deaths", Bulletin of the Department of Health, Kentucky, January, 1938, pp. 45I-53.

"The Wothers and Babies Who will Not Colebrate May Day". Bullotin of the Department of Health, Kontucky, May, 1938, pp. $496-97$.

"Infant and Neonatal Deaths in Kentucky", Bulletin of the Dopartment of Hen Ith, Kentucky, November, 1938, pp. 543-46.

"Deaths Among Preschool Children in Kentucky", Bulletin of the Department of Fealth, Kentrucky, December, 1939, 00. 11I-13.

"Deaths From Diseases of the Respiratiory System in Preschool Group: Kentuoky - 1932-1937", Bulletin of the Department of Hoalth, Kentucky, January, 1940, pp. 123-24.

"A Deolining Infant liortality Rate", Bulletin of the Department of Health, Kentucky, October, 1940, pp. 219-21.

"Blood, Sweat and Tears", Bulletin of the Department of Hoalth, Kentuoky, Decernber, 1941, pp. 378-380.

Crittenden, C. B. and Neal, Dorothy Jane, "Prevalence of Diarrhea and Enteritis and Dysentery in the Younger Age Groups", Bulletin of the Department of Health, Kentuoky, Ootober, 1941, pp. 355-57.

Federal Security Agency, Charts on Infant and Childhood Mortality, Births, Maternal Mortality, 1945, U.S. Childrens Bureau, Washington.

Hirschfold, Gorhard and Strow, Carl W. "Comparative Health Faotors Among the States", American Sooiological Review, Fobruary, 1946, pp. $42-52$.

Pediatric Progress Study. Study of Child Health Services. Washingtons Amerioan Acadeny of Pediatrics, 1947.

Report of Committen on Medical Eeonomics of the Kentuoky State Medical Assoolation. Louisville: Denartment of Realth, Commonwealth of Kentucky. August, 1939. 
Williams, Charles L. Jr., Hubbard, John P., Bain, Katherine. "Community Health Services in Elght Selected States", American Journal of Eublic Health, Jamiary, 1949, pp. 75-81. 